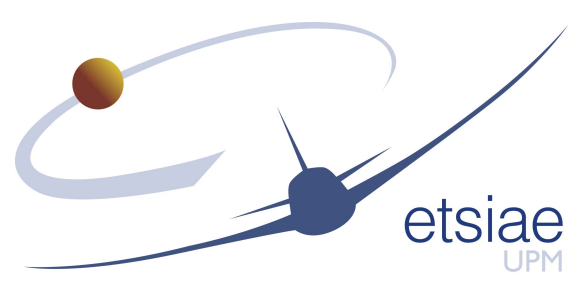

\author{
UNIVERSIDAD POLITÉCNICA DE MADRID \\ ESCUELA TÉCNICA SUPERIOR DE INGENIERÍA AERONÁUTICA \\ Y DEL ESPACIO
}

\title{
ANÁLISIS DEL FLUJO EN ATOMIZADORES DE PRESIÓN CON GIRO
}

\section{Tesis Doctoral}

Ángel Pereña Tapiador

Ingeniero Aeronáutico 

DEPARTAMENTO DE MECÁNICA DE FLUIDOS

Y PROPULSIÓN AEROESPACIAL

ESCUELA TÉCNICA SUPERIOR DE INGENIERÍA AERONÁUTICA

Y DEL ESPACIO

\title{
ANÁLISIS DEL FLUJO EN ATOMIZADORES DE PRESIÓN CON GIRO
}

\author{
Autor \\ Ángel Pereña Tapiador \\ Ingeniero Aeronáutico
}

Director de la Tesis

Francisco José Higuera Antón

Doctor Ingeniero Aeronáutico 



\section{Índice}

Índice $\quad$ I

Agradecimientos $\quad$ V

Resumen

$\begin{array}{lll}\text { Abstract } & \text { IX }\end{array}$

1 Introducción y revisión bibliográfica $\quad 1$

1.1 Descripción de los atomizadores de presión con giro . . . . . . . . . . 3

1.2 Influencia de las propiedades del líquido y del gas . . . . . . . . . . . . . . 4

1.3 Revisión bibliográfica . . . . . . . . . . . . . . . . . . . 5

2 Aproximación de flujo no viscoso irrotacional $\quad 17$

2.1 Formulación del problema . . . . . . . . . . . . . . . . . . 17

2.2 El flujo sobre un rebosadero y a la salida de la cámara de un atomizador:

la condición de caudal máximo . . . . . . . . . . . . . . . . . . . . 23

2.2.1 El flujo sobre un rebosadero . . . . . . . . . . . . . . . . 23

2.2.2 El flujo a la salida de la cámara de un atomizador . . . . . . . . . 25

2.3 Revisión de la aproximación cuasi-unidireccional . . . . . . . . . . . . 26

2.3.1 Coeficiente de descarga y espesor de la lámina . . . . . . . . . . . 27

2.3.2 Semiángulo de la lámina cónica . . . . . . . . . . . . . . . . . . 35

2.4 Estudio numérico . . . . . . . . . . . . . . . . . . . . . . . . . . . . . . 39

2.4.1 El método de los elementos de contorno . . . . . . . . . . . . . . 40

2.4.2 Formulación del método . . . . . . . . . . . . . . . . . . 40

2.4 .3 Simulación . . . . . . . . . . . . . . . . . . 43

2.4.4 Resultados del estudio numérico . . . . . . . . . . . . . . . . 46

2.5 Propagación de ondas en la superficie del núcleo de aire . . . . . . . . . . . 55 
2.5 .1 Ondas en la cámara . . . . . . . . . . . . . . . . . . 57

2.5 .2 Ondas cerca del orificio de salida . . . . . . . . . . . 58

2.5.3 Reflexión y transmisión de ondas en la región de transición entre la cámara y el orificio de salida . . . . . . . . . . . . . . . . 60

2.6 Propagación de ondas en la superficie del núcleo de aire en una cámara de sección lentamente variable . . . . . . . . . . . . . . . . . . . 63

3 Efecto de la viscosidad $\quad 71$

3.1 Formulación de un modelo cuasi-cilíndrico . . . . . . . . . . . . . . 73

3.1 .1 Condición de contorno a la entrada . . . . . . . . . . . . . 76

3.1 .2 Condición de contorno a la salida . . . . . . . . . . . 76

3.1.3 Estimación del ángulo de la lámina cónica . . . . . . . . . . . 80

3.1 .4 Simulación numérica . . . . . . . . . . . . . . . . . . 82

3.2 Resultados . . . . . . . . . . . . . . . . . . . . . . . . 83

3.2.1 Efecto de la constante del atomizador . . . . . . . . . . . 84

3.2.2 Efectos de otros parámetros geométricos $\left(R_{s}, \ell_{1}, \ell_{2}\right) \ldots \ldots . . .87$

3.2 .3 Efectos del número de Reynolds . . . . . . . . . . . . . . . 90

4 Forma y estabilidad de la lámina líquida $\quad 93$

4.1 Lámina líquida estacionaria . . . . . . . . . . . . . . . . . . . 94

4.2 Estabilidad de la lámina líquida . . . . . . . . . . . . . . . . 100

4.3 Inestabilidad de Rayleigh-Taylor . . . . . . . . . . . . . . . . . . 108

4.3.1 Máximo ritmo de crecimiento de la inestabilidad de Rayleigh-Taylor 109

4.3.2 Tiempo de residencia en la región de máxima aceleración . . . . . . 111

4.3 .3 Criterio de inestabilidad . . . . . . . . . . . . . . . . 112

4.4 Inestabilidad centrífuga debida al giro del líquido . . . . . . . . . . . 113

4.4.1 Perturbaciones de longitud de onda grande . . . . . . . . . . . . . 114

4.4 .2 Perturbaciones de longitud de onda pequeña . . . . . . . . . . . . . 114

$\begin{array}{lll}5 & \text { Conclusiones } & 117\end{array}$

A Propagación de ondas en la superficie del núcleo de aire $\quad 127$

A.1 Ondas en la cámara . . . . . . . . . . . . . . . . . . . . . . . . 130

A.2 Ondas cerca del orificio de salida $\ldots \ldots \ldots \ldots \ldots \ldots$

B Propagación de ondas en la superficie del núcleo de aire a través de una región de transición compacta 
C Propagación de ondas en la superficie del núcleo de aire en una cámara $\begin{array}{lc}\text { de sección lentamente variable } & 137\end{array}$

C.1 Solución estacionaria . . . . . . . . . . . . . . . . . . . . . . . 138

C.2 Problema dependiente del tiempo . . . . . . . . . . . . . . . . . 140

D Lámina líquida estacionaria $\quad 149$

$\begin{array}{lll}\text { E Estabilidad de la lámina líquida } & 157\end{array}$

$\begin{array}{ll}\text { Bibliografía } & 165\end{array}$ 


\section{Agradecimientos}

Cuando cuatro años atrás el doctor Manuel Rodríguez, quien era mi jefe en la empresa en la que trabajaba, además de catedrático de esta escuela, me comentó la posibilidad de que realizara los estudios de doctorado, no tenía ni idea de dónde me metía. Pero hasta que no tocas el fuego no te puedes imaginar cuánto te va a doler. Yo no sabía lo que era el fuego. Sin embargo, tuve la suerte de contar con el doctor Francisco Higuera como director de mi tesis. Supongo que él no tuvo tanta conmigo. En verdad aseguro que nunca he conocido a una persona con unos conocimientos tan profundos en la materia. No solo cuenta con todo mi agradecimiento, sino con mi más profunda admiración y mi más absoluto respeto. Él es el verdadero autor de esta tesis.

Hace diez años mi campo de acción era el de las estructuras aeronáuticas. Por ciertas circunstancias, acabé enrolándome en el equipo de fluidodinámica de Manuel Rodríguez. A él, a Rafael Rebolo y a todo su grupo les debo mucho. Los años que pasé trabajando con ellos siempre los recordaré como una etapa muy especial. Fueron numerosos los proyectos que compartimos juntos, y bastante lo que pude aprender.

También aquí en la escuela me he topado con varias personas que me han ofrecido su ayuda, en especial mis compañeros José Miguel, Santiago Enrique, Alonso y José Manuel, que en determinadas ocasiones me han tenido que asistir con alguna que otra dificultad.

Han sido abundantes los momentos difíciles que he pasado, y que no hubiera superado si no hubiera sido por la gente que me rodea. A ellos les debo también mi agradecimiento, sobre todo a mis padres, María Cristina y Ángel, y a mi hermana Cristina, que cuentan con todo mi amor. Y también a mis amigos y a toda la gente que me ha apoyado: Carlos, Damian, Ricardo, Pedro, Juanjo, Pilar, Wendy, Jesús, Alejandro, Ángel, Juancho, Bruno, Patrick Anthony, Roberto, Silvia, Andrei, Valentín, y a muchos más que aquí me dejo. Mención especial merece mi amigo Paco, también ingeniero, con quien he compartido agradables charlas sobre ingeniería, y sobre otras muchas cosas, y con quien siempre he podido contar. 
Y por último, quería recordar a dos mujeres que han sido muy importantes para mí no solo durante el periodo de desarrollo de mi tesis, sino durante mi vida, y a las que quiero inmensamente. Una de ellas es Irene, con quien he compartido muchos años de mi existencia, y con la que he crecido como persona. Siempre he contado con su apoyo. La otra es Urica, que aunque la conocí hace menos de un año, entró en mi vida como un ciclón, y me ha insuflado gran cantidad de fuerza y de ganas de seguir adelante. Sin ella me hubieran resultado mucho más difíciles estos últimos meses tan intensos.

Esta tesis es de todos vosotros. Porque sin vosotros nunca la hubiera podido realizar. Gracias. 


\section{Resumen}

Los atomizadores de presión con giro son uno de los tipos más comunes de atomizador. En ellos el líquido es inyectado en una cámara axilsimétrica a través de unos canales tangentes a su pared lateral que imprimen una velocidad de giro al líquido, dando lugar a un torbellino hueco con un núcleo de aire que se extiende a lo largo del eje de la cámara. El líquido, tras atravesar una región convergente, fluye a través de un orificio de salida concéntrico a la cámara, formando una lámina anular. Esta lámina, al abandonar el orificio, se expande radialmente, tomando inicialmente una forma cónica, y forma ligamentos que se rompen en gotas. Los problemas fluidodinámicos asociados a la operación de los atomizadores de presión con giro son complejos. La obtención de información sobre el comportamiento del flujo mediante ensayos experimentales es complicada, debido al pequeño tamaño de los atomizadores y a las dificultades de acceso óptico. El cálculo numérico directo del flujo tampoco está libre de complejidad, debido a la presencia de varios procesos simultáneamente, cuya influencia en las características del atomizador no es fácil de identificar. En estas condiciones, se ha enfocado el estudio del problema mediante la formulación de modelos aproximados de procesos o grupos de procesos individuales, que puedan analizarse con relativa facilidad y proporcionar información relevante.

Primeramente, se ha realizado un estudio de una aproximación no viscosa del flujo. Para analizar la influencia de los diferentes parámetros geométricos del atomizador, se ha estudiado numéricamente el problema, para lo que se ha construido un modelo basado en el método de los elementos de contorno. Este modelo nos ha permitido determinar cómo varían las principales características del flujo estacionario para varias configuraciones geométricas del atomizador, mostrando las discrepancias con los resultados obtenidos previamente por otros autores mediante modelos irrotacionales más simplificados. También se ha estudiado el problema no estacionario de la propagación de ondas en la superficie del núcleo de aire, obteniendo las velocidades de propagación de las mismas en las diferentes regiones de la cámara del atomizador.

A continuación, se han analizado los efectos de la viscosidad en el flujo interno del atomizador a altos números de Reynolds. Para ciertas configuraciones del atomizador, las 
capas límite que aparecen pueden cubrir gran parte del espesor de la lámina líquida, lo que influye en las condiciones del flujo y resta validez a los resultados obtenidos mediante modelos irrotacionales. Para estudiar este efecto, se ha analizado el problema viscoso empleando una aproximación cuasi-cilíndrica que ha permitido obtener las principales características del flujo a un coste computacional mucho menor que el que supondría la resolución de las ecuaciones de Navier-Stokes completas. Los resultados obtenidos muestran la importancia del papel jugado por las capas límite. Éstas causan un aumento del espesor de la lámina líquida al final del orificio de salida respecto al calculado por las aproximaciones irrotacionales, así como una disminución de la sobrepresión requerida y del semiángulo de la lámina cónica generada.

Finalmente, se ha estudiado la forma y la estabilidad de la lámina líquida generada a la salida del atomizador. Si ésta no se rompe antes, inicialmente toma una forma cónica, pues en la región próxima a la salida del atomizador los efectos de la tensión superficial son despreciables, y se cierra aguas abajo cuando dichos efectos se hacen importantes. Si la velocidad relativa entre el líquido y el gas que lo rodea es suficientemente alta, se excita una inestabilidad de Kelvin-Helmholtz, que produce un novimiento oscilatorio en la lámina similar al de una bandera. Esta inestabilidad da lugar a aceleraciones transitorias perpendiculares a la lámina, que pueden derivar en una inestabilidad secundaria de Rayleigh-Taylor, la cual es la causante de la formación de ligamentos que posteriormente se rompen en gotas. El estudio de la inestabilidad de Rayleigh-Taylor nos ha permitido estimar la distancia a la que se desintegra la lámina líquida. 


\section{Abstract}

Pressure swirl atomizers are one of the most common types of atomizer used in a large variety of industrial applications. These atomizers generate an axisymmetric sheet of rotating liquid that rapidly breaks into ligaments and then drops. To generate this sheet, liquid is injected into a cylindrical chamber through tangential slots that induce a swirling flow in the chamber, in the form of a hollow vortex with an air core extending along the axis of the chamber. This flow converges towards a central exit orifice in a process that intensifies the rotation of the liquid, which emerges from the chamber as an annular rotating sheet that rapidly becomes conical when its radial motion ceases to be restricted by the chamber wall. The flow associated with the operation of pressure swirl atomizers is complex. The experimental tests performed in order to obtain information on the behaviour of the flow are dificult, due to the small size of the atomizers and the difficulties of optical access. Direct numerical techniques are also complicated, due to the presence of several simultaneous processes, whose influence on the characteristics of the atomizer is not easy to identify. In these conditions, our study of the problem has proceeded through the formulation of approximate models of processes or groups of individual processes that can be analyzed with relative ease and provide relevant information.

A non-viscous, irrotational, approximation to the flow has been studied. In order to analyze the efects of the geometry of the atomizer, a boundary element method (BEM) model has been developed to simulate the irrotational flow. The influence of several geometrical parameters on the steady flow has been determined and compared with the results of previous researchers. In addition, wave propagation along the surface of the air core has also been studied.

If the viscosity of the liquid is considered, boundary layers develop at the walls of the chamber. These boundary layers may cover a substantial part of the sheet in typical conditions, which limits the validity of irrotational models. A quasi-cylindrical approximation for viscous swirling flows slowly varying in the axial direction has been used to describe the internal flow in an atomizer chamber. The results show that this approximation captures many features of the flow in the chamber at a fraction of the computational 
cost of a full Navier-Stokes simulation. The results are in good agreement with previous simulations and experiments.

The effect of the surface tension is small in the liquid sheet that emerges from the atomizer, so that the sheet rapidly takes a conical shape. However, surface tension effects become important in a certain region far downstream of the atomizer where the sheet departs from a cone. If the relative velocity between the liquid and the surrounding gas is high enough, a Kelvin-Helmholtz instability is excited that causes an undulatory motion of the sheet, so that the liquid is alternatively accelerated towards one or the other side of the sheet. In these conditions, the interface between the liquid and the gas is potentially unstable. This instability (Rayleigh-Taylor instability) is responsible for the formation of ligaments, and determines the distance at which the liquid sheet disintegrates. 


\section{Capítulo 1}

\section{Introducción y revisión bibliográfica}

El proceso de atomización consiste en disgregar un volumen de líquido en gotas. Es de gran importancia en numerosos procesos industriales y otras aplicaciones. Entre sus usos se puede citar la dispersión de combustibles líquidos para su posterior combustión, la aplicación de productos químicos en plantaciones agrícolas, la pulverización de pintura, el secado por aspersión, la extinción de incendios, la refrigeración en aplicaciones nucleares, los aspersores de jardín, los pulverizadores capilares, y muchos otros. La combustión de combustibles líquidos en motores diésel, motores de ignición por chispa, turbinas de gas, motores cohete y hornos industriales es altamente dependiente del nivel de atomización, pues un incremento del área de la superficie específica del combustible líquido da lugar a un incremento del grado de mezclado y evaporación del mismo. En estas aplicaciones, una reducción del tamaño medio de las gotas de combustible origina mayores niveles de transferencia de calor, un encendido más fácil, una combustión mejor y una menor emisión de contaminantes. Sin embargo, en otras aplicaciones como el rocío de cosechas, la formación de gotas muy pequeñas daría lugar a que, bajo ciertas condiciones meteorológicas, las mismas fueran arrastradas por el aire.

Los dispositivos encargados de pulverizar el líquido se llaman atomizadores, y los hay de muchos tipos. El spray se puede producir de varias maneras. En general, el proceso de atomización se logra mediante la desintegración de un chorro o lámina líquida debida a la energía cinética del líquido, a la exposición del mismo a un gas a alta velocidad o como resultado de una energía mecánica aplicada externamente, por ejemplo por un dispositivo vibratorio. La forma y la penetración del spray, así como las distribuciones de la velocidad y el tamaño de las gotas en función del espacio y del tiempo, estarán determinadas por el desarrollo del chorro o de la lámina líquida, y el crecimiento de pequeñas perturbaciones que dan lugar a su desintegración en ligamentos y posteriormente 
en gotas. Estos parámetros también se verán afectados por la geometría del atomizador y por las propiedades físicas del líquido y del gas en el que se inyecta.

El concepto más sencillo de atomización consistiría en la desintegración de un chorro líquido que emerge de un orificio circular, con la componente principal de la velocidad en dirección axial y flujo laminar. De acuerdo con Rayleigh (1879), el chorro se rompe en gotas debido al crecimiento de pequeñas perturbaciones. Cuando el chorro emerge del orificio, la componente radial de la velocidad deja de estar restringida por las paredes del atomizador, pasando a estar sujeta sólo por la tensión superficial del líquido. Cuando se supera esta tensión superficial, el chorro se desintegra. La viscosidad coarta el crecimiento de las perturbaciones, dando lugar a un retraso en la desintegración del chorro, por lo que la atomización ocurre en regiones más lejos aguas abajo del orificio, donde la velocidad relativa entre el líquido y el gas es menor, por lo que el tamaño de las gotas que se forman será mayor. Además un incremento en la viscosidad del líquido deriva en un aumento de la longitud de onda de las perturbaciones más inestables, la cual determina el tamaño de las gotas.

En muchas aplicaciones, para conseguir la dispersión deseada de las gotas en el gas, se busca generar un spray con forma plana o cónica. Para que la lámina plana o cónica se extienda, se requiere una velocidad mínima del líquido para vencer las fuerzas de tensión superficial que tienden a contraer la lámina. Cualquier perturbación inicial presente en la lámina crecerá debido a las inestabilidades aerodinámicas, dando lugar a perforaciones en la misma cuando ésta se expande y su espesor disminuye. Dichas perforaciones se expanden y se juntan, haciendo que la lámina líquida se rompa en ligamentos. Estos ligamentos a su vez se romperán para formar gotas, cuyo diámetro variará ampliamente debido a la variación del diámetro de los ligamentos. Algunas de las gotas más grandes generadas de esta forma se romperán para dar lugar a gotas más pequeñas. El tamaño medio de las gotas producidas dependerá principalmente del espesor inicial de la lámina líquida, de su velocidad relativa al gas que la rodea, y de la viscosidad y tensión superficial del líquido. Si la lámina líquida se mueve a alta velocidad, se puede desintegrar en ligamentos en ausencia de formación de perforaciones por mecanismos ondulatorios, donde las ondas son generadas por interacción aerodinámica con el gas que rodea a la lámina. Si la velocidad del líquido es muy alta, la lámina se desintegra próxima al lugar de donde emerge. Sin embargo, para todas estas formas de desintegración, el último proceso de atomización consiste en la rotura de los ligamentos originados para formar gotas de acuerdo con el mecanismo de Rayleigh. 


\subsection{Descripción de los atomizadores de presión con giro}

Básicamente, el requerimiento para generar un spray consiste en generar una alta velocidad relativa entre el líquido que se desea atomizar y el gas que lo rodea. Esto se puede conseguir de varias maneras. En el caso de los atomizadores de presión, se aplica una alta presión sobre el líquido, que es descargado al ambiente a través de una pequeña abertura. La energía debida a esta presión se convierte en energía cinética, dando lugar a una alta velocidad del líquido, mientras que el gas que ocupa el ambiente presenta un movimiento lento en relación con el del líquido.

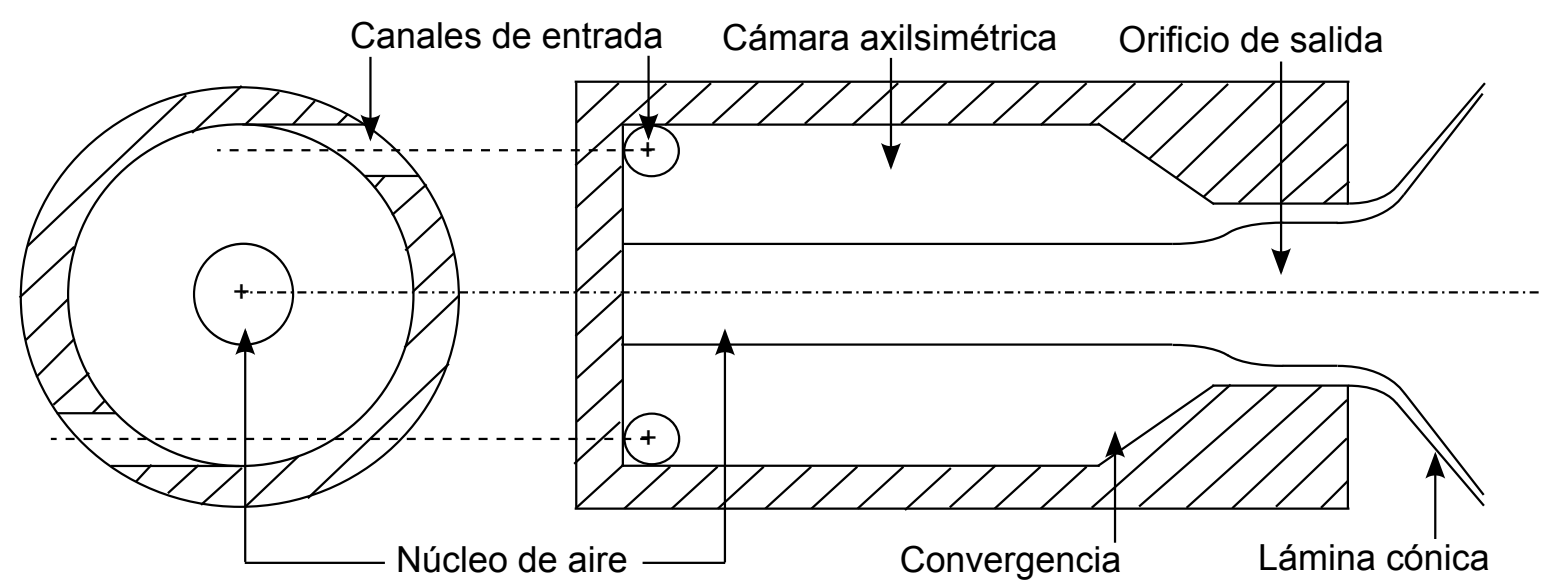

Figura 1.1 - Esquema de un atomizador de presión con giro con 2 canales de entrada.

Los atomizadores de presión con giro generan una lámina cónica giratoria de un líquido que rápidamente se rompe en ligamentos y posteriormente en gotas. Para producir esta lámina, se inyecta el líquido en una cámara axilsimétrica a través de unos canales tangentes a la pared de dicha cámara, con el objetivo de proporcionar un movimiento de giro al fluido, en forma de un torbellino hueco con un núcleo de aire que se extiende a lo largo del eje de dicha cámara. Este giro produce un gradiente radial de presión que equilibra a las fuerzas centrífugas. Tras recorrer la cámara, el líquido converge hacia un orificio de salida concéntrico con el eje de la misma, y emerge formando una película giratoria anular que enseguida se vuelve cónica cuando su movimiento radial deja de estar restringido por las paredes del atomizador. Dependiendo de la aplicación, el ángulo de este cono puede variar desde $30^{\circ}$ hasta casi $180^{\circ}$ (Lefebvre 1989). Los tamaños más finos de las gotas que se forman al romperse la lámina cónica se producen cuanto mayores son la presión 
suministrada y el ángulo del cono. Un esquema de la configuración de estos atomizadores se muestra en la figura 1.1.

Las principales características del flujo en un atomizador son el espesor de la lámina que forma el líquido cuando sale del atomizador, el ángulo de la lámina cónica que se forma a cierta distancia aguas abajo, y el coeficiente de descarga, que es el caudal del líquido inyectado en el atomizador escalado con el producto del área del orificio de salida y la raiz cuadrada del doble de la sobrepresión en la cámara dividida por la densidad del líquido. Todas estas características dependen del flujo giratorio en el interior de la cámara, que es bastante complejo.

\subsection{Influencia de las propiedades del líquido y del gas}

Las características del spray generado por el atomizador están influidas por la densidad, la tensión superficial y la viscosidad del líquido a atomizar. Sin embargo, la importancia de la densidad disminuye debido a que la mayoría de los líquidos empleados en aplicaciones de atomización tienen valores similares de la misma. Además, los datos disponibles de su efecto en las características del spray sugieren que su influencia es muy pequeña (Lefebvre 1989).

Un efecto resultante de la atomización es el incremento del área de la superficie del líquido. Antes de que se produzca la rotura, dicha área será la de la lámina líquida en el momento en que emerge del orificio, y después de la atomización será la suma de las áreas de las superficies de todas las gotas. Esta relación es importante en fenómenos como la evaporación de las gotas. Así pues, la tensión superficial será relevante en la atomización, pues representa la fuerza que resiste el incremento del área de la superficie del líquido.

En muchos aspectos, la propiedad del líquido más importante en el proceso de atomización es la viscosidad, ya que no sólo afecta al tamaño de las gotas generadas, sino también al caudal, para una sobrepresión dada, y a la forma del spray. Un incremento en la viscosidad disminuye el número de Reynolds y atenúa la formación de inestabilidades en la lámina líquida, lo que da lugar a un retraso en la desintegración de la misma y a un tamaño mayor de las gotas formadas. El caudal puede aumentar para incrementos moderados de la viscosidad, debido al incremento del espesor de la lámina líquida en el orificio de salida. Sin embargo, para altos valores de la viscosidad el caudal suele disminuir cuando ésta aumenta (Lefebvre 1989). Un aumento de la viscosidad también da lugar a una disminución del ángulo de la lámina cónica que se forma, llegando a colapsar en un chorro recto para muy altos valores de la misma que da lugar a gotas relativamente 
grandes. Además, un incremento en la viscosidad del líquido da lugar a un aumento de las pérdidas por viscosidad, luego la energía disponible será menor. Así pues, el incremento de la viscosidad del líquido tiene un efecto adverso en el proceso de atomización.

A su vez, las características del spray generado también se verán afectadas por las propiedades del gas del ambiente. El ángulo que forma la lámina cónica líquida disminuye cuando aumenta la densidad del gas, hasta que se alcanza un ángulo mínimo más allá del cual las variaciones en la densidad del gas apenas tienen efecto (Lefebvre 1989). La densidad del gas también tiene un efecto importante en el tamaño de las gotas que se generan cuando se desintegra la lámina líquida. Además, la presión y la temperatura del gas pueden variar ampliamente, sobre todo en ciertas aplicaciones como sistemas de combustión.

\subsection{Revisión bibliográfica}

Como indicamos antes, las principales características del flujo en un atomizador son el espesor de la lámina que forma el líquido cuando sale del atomizador, el ángulo de la lámina cónica que se forma a cierta distancia aguas abajo, y el coeficiente de descarga, las cuales dependen del flujo giratorio en el interior de la cámara, que es muy complejo. Sin embargo, se ha obtenido mucha información usando modelos irrotacionales de este flujo. Varios autores (Abramovich 1944, Novikov 1948, Taylor 1948, Giffen \& Muraszew 1953, Nieuwkamp 1985, Bayvel \& Orzechowski 1993; una revisión se incluye en Chinn 2009) han estudiado el problema con la aproximación adicional de flujo cuasi-unidireccional, en el sentido de que consideran que la componente radial de la velocidad del líquido es nula a lo largo del orificio de salida, y la componente axial es uniforme. En estas condiciones las incógnitas del problema son, para una cierta presión a la que se inyecta el líquido en la cámara, el caudal suministrado, el espesor de la lámina líquida en el orificio de salida, y la velocidad axial del líquido en dicho orificio. Una primera relación entre estas tres variables la obtienen igualando el caudal al producto de la velocidad axial del líquido en el orificio de salida por el área de la sección de la lámina anular líquida en dicha región. Otra relación entre la velocidad axial y el espesor de la lámina líquida en el orificio la suministra la ecuación de Bernoulli aplicada en la superficie libre del líquido en el orificio de salida. Sin embargo, sólo se dispone de dos ecuaciones y se cuenta con tres incógnitas. La condición adicional que emplean estos modelos es que el flujo en el orificio de salida ha de ser crítico, en el sentido de que la velocidad axial del líquido en dicho orificio ha de coincidir con la velocidad de propagación de ondas en la superficie libre, lo que fue demostrado en los 
estudios realizados por Binnie \& Hookings (1948) y Binnie (1949) para descarga de flujos giratorios a través de una tobera. Para dar cuenta de esta condición varios autores (Taylor 1948, Giffen \& Muraszew 1953, Bayvel \& Orzechowski 1993) imponen que el caudal ha de ser máximo, obteniendo una ecuación adicional resultado de igualar a cero la derivada de la expresión del caudal como función del espesor de la lámina líquida en el orificio. Otros autores (Nieuwkamp 1985) consideran el problema del flujo en un atomizador de presión con giro análogo al del flujo sobre un rebosadero, y aplican la expresión para la velocidad del líquido en la cresta del rebosadero a la velocidad axial del líquido en el orificio de salida del atomizador, sustituyendo la aceleración de la gravedad por la aceleración centrífuga debida al giro del líquido. De todos estos modelos resulta que las características del flujo dependen únicamente de un parámetro adimensional, la constante del atomizador, que se define como el área de la sección transversal de los canales a través de los cuales se inyecta el líquido en la cámara escalada con el producto de los diámetros de la cámara y del orificio de salida, obteniendo que al aumentar este parámetro aumentan el coeficiente de descarga y el espesor de la lámina líquida en el orificio y disminuye el ángulo de la lámina cónica que se forma a la salida.

El problema irrotacional también ha sido objeto de estudios numéricos con el fin de analizar la influencia en las características del flujo de los demás parámetros geométricos del atomizador, aparte de la constante del atomizador. Tal es el caso de las simulaciones numéricas llevadas a cabo por Park \& Heister (2006), en las que realizaron un modelo basado en el método de los elementos de contorno (BEM, Boundary Element Method).

Los resultados obtenidos en los estudios del problema irrotacional serán de aplicación cuando la viscosidad del líquido sea muy pequeña. Sin embargo, debido a la viscosidad se generan capas límite en las paredes del atomizador y alrededor del núcleo de aire. Taylor (1948) comentó que las capas límite que aparecen a cada lado de la lámina líquida anular que emerge del orificio de salida pueden cubrir una parte importante del espesor de la lámina en condiciones típicas, lo que limita la validez de los modelos irrotacionales. Estas capas límite fueron estudiadas mediante métodos integrales por Taylor (1950a), Binnie \& Harris (1950) y Cooke (1952).

Tanto Taylor (1950a) como Cooke (1952) estudiaron el crecimiento de la capa límite que se forma sobre la superficie interior de un cono lleno de un líquido en rotación, y la velocidad con la cual el líquido en la capa límite fluye hacia el vértice. Como simplificación supusieron que la componente axial de velocidad fuera de la capa límite puede ser despreciada, considerando en esta región únicamente el movimiento de giro. En la capa límite sí se presentará dicha componente axial, debido a que el flujo es dirigido hacia el 
vértice por bombeo de Ekman. El flujo estudiado difiere del flujo en un atomizador de presión con giro en que, en este último caso, la velocidad fuera de la capa límite también presenta una componente paralela a las paredes. Sin embargo, en la mayoría de los atomizadores dicha componente es comparativamente pequeña respecto a la componente azimutal, excepto cerca del orificio de salida. Así pues, en la región donde se genera la capa límite la aproximación usada por Taylor (1950a) y Cooke (1952) es suficientemente buena, y el espesor de la capa límite a un radio correspondiente al del orificio de salida puede proporcionar una idea de si la mayoría del flujo sale por el orificio desde la capa límite o desde el exterior de la misma. El trabajo de estos autores muestra que gran parte del líquido, o incluso todo, dependiendo de las propiedades del mismo, abandona el orificio de salida a través de la capa límite.

Por su parte, Binnie \& Harris (1950) estudiaron el flujo de un líquido viscoso giratorio a través de una tobera convergente-divergente, pero a diferencia de Taylor (1950a) y Cooke (1952) sí tuveron en cuenta la componente axial de velocidad fuera de la capa límite. El ángulo de dicha tobera cónica es pequeño, de forma que la componente radial de la velocidad puede ser despreciada. En el estudio de la corriente principal fuera de la capa límite se obtuvo que la componente axial de la velocidad es uniforme en cada sección transversal, siendo su valor en la garganta de la tobera el correspondiente a la velocidad de una onda moviéndose en la superficie del núcleo de aire. Se consideraron los efectos de la tensión superficial sobre el núcleo de aire y se mostró que los mismos son despreciables. Los resultados obtenidos para el caso de flujo con giro y sin giro se compararon con los obtenidos por Taylor (1950a), donde no había velocidad axial en el flujo principal, obteniéndose menores espesores para la capa límite que en este último caso.

Los anteriores estudios permiten entender la evolución de la capa límite en las paredes del atomizador, pero no consideran la capa límite que se forma alrededor del núcleo de aire. Los experimentos realizados por Binnie, Hookings \& Kamel (1957) sobre flujos giratorios en toberas de Perspex muestran valores altos de la velocidad axial del líquido en la región próxima al núcleo de aire, lo que confirma la presencia de la capa límite alrededor del núcleo de aire debido al bombeo de Ekman sobre el líquido en la capa límite formada sobre la pared de la cámara opuesta al orificio de salida, y del giro del fluido cuando alcanza la superficie libre y su redirección hacia la salida. Estos experimentos también muestran una disminución en el radio del núcleo de aire y en la sobrepresión en la cámara y, por tanto, un aumento del coeficiente de descarga, respecto a los valores estimados por la teoría irrotacional, efecto atribuido a la reducción de la velocidad de giro debida a la 
viscosidad.

Para entender la formación de la capa límite en la base de la cámara y alrededor del núcleo de aire son importantes los estudios de Burggraf, Stewartson \& Belcher (1971) y Bloor \& Ingham (1977). Estos autores estudiaron la capa límite laminar que se forma sobre un disco circular cuyo eje es concéntrico con el de un torbellino de velocidad azimutal $w \sim r^{-1}$. En el estudio de Bloor \& Ingham (1977) se consideró además un flujo radial hacia el eje de la forma $v \sim r^{-m}$. Realizaron un estudio numérico de las ecuaciones de capa límite comenzando por la periferia del disco y avanzando hacia el eje hasta mostrar las características del flujo terminal. También propusieron una solución asintótica de las ecuaciones de capa límite cerca del eje, que se ajusta bastante bien a los resultados numéricos que obtuvieron. Dicha solución asintótica presenta una estructura de doble capa. En las proximidades del disco, una región interior que se extiende sobre el disco una distancia del orden del radio del mismo es dominada por un flujo radial hacia el eje debido al fuerte gradiente de presión. La velocidad azimutal en esta región, aunque grande, es pequeña comparada con la velocidad radial. Sobre esta zona hay una región exterior donde el flujo es principalmente no viscoso, y donde la velocidad azimutal del fluido aumenta desde cero hasta la de un torbellino potencial según la distancia al disco aumenta de cero a infinito.

Por otro lado, Dumouchel et al. (1992) estudiaron el problema del flujo en la capa límite en el orificio de salida de un atomizador de presión con giro. Para ello adaptaron el análisis del flujo en la capa límite sobre un disco plano circular realizado por Bloor \& Ingham (1977) al flujo giratorio en una tobera cónica. Definiendo el parámetro $K$ como la relación entre las componentes azimutal y radial de la velocidad en la base del cono, observaron que los perfiles de velocidad radial en la capa límite varían muy poco para $K>5$, y que los perfiles de velocidad azimutal son totalmente independientes de $K$. Variando el valor de la presión de inyección, encontraron que $K$ es función únicamente de la geometría de la tobera. Determinaron las relaciones entre el espesor de la capa límite y el espesor de la lámina líquida, y entre el caudal en la capa límite y el caudal total, observando que ambas relaciones son función del radio del orificio de salida, del radio de los canales de entrada a la tobera y de la presión de inyección. Concluyeron que la capa límite constituye una parte importante del flujo total cuando estos tres parámetros son relativamente pequeños. Cuando aumentan, la importancia del flujo en la capa límite disminuye comparada con el flujo principal, pudiendo llegar a despreciarse en algunos casos.

Una variedad de técnicas experimentales y numéricas se han empleado para caracte- 
rizar el flujo interno en la cámara de un atomizador de presión con giro. Se han realizado experimentos con varias configuraciones geométricas, propiedades del líquido y diferentes valores de la presión de inyección y/o del caudal (Dombrowski \& Hasson 1969, Rizk \& Lefebvre 1985, Horvay \& Leuckel 1986, Suyari \& Lefebvre 1986, Kim et al. 2009), que han permitido clarificar los efectos de estos parámetros en las características del atomizador, y que han dado lugar a una extensión de los resultados predichos por la teoría irrotacional. Se han empleado modelos a escala aumentada, fabricados con materiales transparentes, que permiten el acceso óptico y la obtención de medidas con mayor resolución espacial mediante velocimetría laser Doppler, velocimetría de imagen de partículas, fluorescencia inducida por laser y técnicas de vídeo de alta velocidad (Horvay \& Leuckel 1986, Holtzclaw et al. 1997, Wang et al. 1999, Donjat et al. 2003). Estos experimentos han revelado la complejidad del flujo en la cámara, con regiones con velocidades axial y azimutal muy diferentes, recirculación, comportamiento no estacionario, ausencia de axisimetría y ondas propagándose por la entrefase entre el líquido y el gas.

Dombrowski \& Hasson (1969) realizaron una serie de experimentos con la idea de determinar los efectos de la constante del atomizador y de las relaciones entre el diámetro de la cámara y el del orificio y entre la longitud y el diámetro de dicho orificio en las características del flujo. Para ello fabricaron una serie de toberas con diferentes geometrías y aplicaron diferentes presiones de inyección. Como resultado de sus ensayos obtuvieron que, para un valor dado de la constante del atomizador, al aumentar la relación entre el diámetro de la cámara y el del orificio, aumenta el coeficiente de descarga y disminuye el ángulo del spray, mientras que al aumentar la relación entre la longitud del orificio y su diámetro disminuyen el coeficiente de descarga y el ángulo del spray.

Por su parte, Rizk \& Lefebvre (1985) derivaron una expresión para el espesor de la lámina líquida que se ajusta bien a los valores medidos experimentalmente en estudios previos, para un amplio rango de dimensiones del atomizador y de presiones de inyección del líquido. Esta expresión muestra que el espesor de la lámina líquida aumenta cuando lo hace el diámetro del orificio de salida y la viscosidad del líquido, y disminuye cuando aumentan la densidad del líquido y la presión de inyección del mismo. Midieron experimentalmente el diámetro medio de las gotas generadas, y vieron que la relación entre dicho diámetro y el espesor de la lámina líquida dado por la anterior expresión se aproxima bastante a los resultados de estudios anteriores. Además obtuvieron una correlación para el coeficiente de descarga en función de las principales dimensiones del atomizador, que también se ajusta satisfactoriamente con los resultados experimentales presentes en la literatura previa. 
Horvay \& Leuckel (1986) llevaron a cabo unos experimentos sobre un modelo a escala aumentada de atomizador. Estudiaron varias geometrías diferentes, y midieron las velocidades por medio de anemometría laser-Doppler. Con el objeto de abarcar un amplio rango de valores de viscosidad, emplearon varios líquidos diferentes para atomizar. En sus experimentos observaron en particular la forma y tamaño del núcleo de aire alrededor del eje de la cámara. Para números de Reynolds bajos el núcleo de aire sólo se extiende a lo largo de la zona próxima al orificio de salida, y desaparece para números de Reynolds muy bajos. Los perfiles de velocidad azimutal observados muestran una distribución de un torbellino potencial, excepto en las proximidades del eje, y apenas varía a lo largo de la cámara, con la región con distribución potencial cada vez menos extensa según se avanza a lo largo de la región convergente de la cámara. Cuanto mayor es el número de Reynolds, más se aproxima la distribución de velocidad azimutal a la de un torbellino potencial. Los perfiles de velocidad axial son bastante planos cerca de la pared, y presentan un máximo en las inmediaciones del eje. Según se avanza aguas abajo de la cámara, la velocidad axial aumenta, debido a la disminución de la sección a lo largo de la región convergente. La desviación observada del comportamiento del flujo frente al de la teoría irrotacional en las inmediaciones del eje de la cámara se debe a la presencia de la capa límite que rodea al núcleo de aire y que genera una disminución de la velocidad azimutal y un aumento de la velocidad axial en su interior.

Los experimentos realizados por Suyari \& Lefebvre (1986) estaban orientados a estudiar la influencia de la presión de inyección en el espesor de la lámina líquida a la salida del atomizador. Para determinar dicho espesor emplearon una técnica consistente en hacer fluir agua a través del atomizador y medir la conductividad eléctrica entre dos electrodos localizados en el orificio de salida. De sus resultados derivaron expresiones para estimar el espesor de la lámina líquida y el diámetro medio de las gotas formadas en función de la caída de presión en el atomizador, las cuales son consistentes con los resultados obtenidos por anteriores investigadores.

Kim et al. (2009) realizaron una serie de experimentos con unos modelos de atomizador con varios valores de la longitud del orificio de salida y del diámetro y longitud de la cámara. La técnica que emplearon para medir el espesor de la lámina líquida en el orificio de salida fue la misma que la utilizada por Suyari \& Lefebvre (1986). Los experimentos los realizaron inyectando agua a varias presiones. Obtuvieron que el espesor de la lámina líquida aumenta con la longitud del orificio de salida, y derivaron una expresión empírica para este espesor en la que aparece dicho parámetro geométrico, ausente en las expresiones dadas anteriormente por Rizk \& Lefebvre (1985) y Suyari \& Lefebvre (1986). También 
estudiaron la formación del núcleo de aire tomando imágenes con una cámara de alta velocidad, lo que les permitió observar las ondas que se forman en la superficie del mismo.

Holtzclaw et al. (1997) estudiaron el flujo en el interior de la cámara de un atomizador mediante técnicas experimentales y numéricas. Sus experimentos los realizaron con un modelo a escala aumentada en el que midieron las velocidades del fluido empleando velocimetría de imagen de partículas (PIV, Particle Image Velocimetry). La región convergente de la cámara presentaba dos configuraciones diferentes, con el fin de analizar la influencia del ángulo de la misma. Para el estudio numérico construyeron un modelo axisimétrico en el que emplearon una técnica de malla adaptativa para capturar la posición de la superficie libre del líquido. Los resultados que obtuvieron mediante estas dos técnicas son muy similares, lo que permite validar el modelo numérico. Observaron que la velocidad azimutal aumenta según nos acercamos al eje de la cámara, pero no presenta su valor máximo en la superficie libre, lo que indica la presencia de la capa límite que rodea el núcleo de aire. Los resultados mostraron también la presencia de regiones de recirculación próximas a la pared de la cámara. En cuanto a la influencia del ángulo de la región convergente, la lámina líquida en el orificio de salida presentaba un mayor espesor, y el ángulo del cono que forma el spray era más pequeño, para la configuración con mayor valor de dicho ángulo.

Para el estudio experimental del flujo en el interior de la cámara de un atomizador realizado por Wang et al. (1999), estos autores fabricaron un modelo a escala aumentada en el que midieron las velocidades del fluido empleando velocimetría de imagen de partículas. Este modelo presentaba valores diversos del área de los canales de entrada, del diámetro del orificio de salida y del ángulo de la región convergente, y los ensayos se hicieron para varios valores del caudal, con el fin de estudiar la influencia de estos parámetros. Observaron que los perfiles de velocidad azimutal y axial eran muy similares en diferentes secciones axiales de la cámara, excepto cerca de los canales de entrada y del orificio de salida. De estos experimentos resultó que tanto al aumentar el caudal como al disminuir el área de los canales de entrada, se producía un aumento del ángulo del spray, debido al incremento de la velocidad azimutal, mientras que al disminuir el diámetro del orificio de salida dicho ángulo disminuía, pues aumentaba la velocidad axial. A su vez, al variar el ángulo de la región convergente se obtuvieron diferentes ángulos del spray, aumentando éste cuando se disminuía el anterior. Este efecto es debido a que la relación entre la longitud y el diámetro del orificio de salida del modelo ensayado era pequeña, por lo que la trayectoria que sigue el fluido cuando sale por el orificio se ve afectada por el ángulo de la región convergente. 
Donjat et al. (2003) estudiaron mediante ensayos experimentales las oscilaciones en la superficie del núcleo de aire generado en el interior de la cámara del atomizador. La visualización y las medidas las realizaron mediante técnicas de anemometría Laser Doppler (LDA, Laser Doppler Anemometry) y cámaras de alta velocidad. Observaron que el flujo interno en la cámara está compuesto de dos chorros helicoidales principales, uno próximo a la pared de la cámara, alimentado directamente por los canales de entrada, y otro en las inmediaciones del núcleo de aire, que presenta una mayor velocidad que el anterior. En estos experimentos también se puso de manifiesto el movimiento de rotación del núcleo de aire. En la entrefase entre el líquido y el gas se observaron perturbaciones que son el resultado de la combinación de varias oscilaciones: un movimiento de precesión del núcleo de aire debido a la difusión del chorro de entrada, unas ondas helicoidales en la superficie del mismo y unas pequeñas fluctuaciones de la superficie asociadas con ondas capilares. Se muestran dos frecuencias principales, la más baja asociada con el movimiento de precesión del núcleo de aire, siendo la otra resultado de la onda helicoidal giratoria. Estos autores además realizaron unas simulaciones numéricas tridimensionales empleando un algoritmo de captura de entrefase del tipo VOF (Volume Of Fluid), en el cual la entrefase viene determinada por la fracción del volumen de cada celda numérica ocupado por el líquido. Dichas simulaciones, realizadas empleando el código comercial FLUENT, confirmaron los resultados experimentales.

En cuanto a los estudios numéricos, Yule \& Chinn (2000) calcularon el flujo interno en la cámara de un atomizador, reproduciendo el generado en modelos a escala aumentada con los que experimentaron, y el de los experimentos realizados por Horvay \& Leuckel (1986). En sus simulaciones, realizadas mediante el código comercial FLUENT en modelos axisimétricos, emplearon un método aproximado para localizar el núcleo de aire. Dicho método consideraba que en todo el dominio computacional sólo había presente una fase, y después identificaba la entrefase uniendo los puntos de la malla en los cuales la presión era la atmosférica. Posteriormente recalculaban la solución generando una nueva malla teniendo en cuenta la entrefase calculada y considerando la misma como una frontera con deslizamiento. Los resultados que obtuvieron concordaban con los datos experimentales disponibles. La componente azimutal de la velocidad en el interior de la cámara presentaba la distribución de un torbellino, excepto en las regiones próximas al núcleo de aire y a la pared. A su vez, el flujo axial se concentraba en estas dos regiones. Sus simulaciones también muestran la aparición de torbellinos de Görtler en la capa límite de la pared de la cámara cuando el tiempo de residencia del flujo cerca de la pared es suficientemente grande. La intensidad de este flujo secundario será mayor cuanto más abrupta sea la 
convergencia entre la cámara y el orificio de salida. Además observaron la formación de ondas en la superficie del núcleo de aire.

Jeng, Jog \& Benjamin (1998) estudiaron el flujo en la cámara de un atomizador y la entrefase entre el líquido y el gas mediante técnicas numéricas y experimentales, determinando las características de la lámina líquida que emerge por el orificio de salida, y comparando sus resultados con las predicciones de la teoría irrotacional y con las correlaciones propuestas por Rizk \& Lefebvre (1985). En los estudios numéricos, realizados sobre un modelo axisimétrico, la posición de la entrefase entre el líquido y el gas es desconocida, y forma parte de la solución. Para la captura de la misma se empleó un método consistente en dos pasos: en el primer paso los nodos de la malla con la que se ha discretizado el dominio fluido se mueven con la misma velocidad que el fluido, de forma que no hay intercambio de masa entre las celdas, y los nodos que se encuentran en la entrefase entre el líquido y el gas siempre permanecen en la misma, y en el segundo paso, para evitar que la malla se deforme excesivamente, se genera una nueva malla y se calculan la masa y la cantidad de movimiento para cada una de las nuevas celdas. Los ensayos experimentales, realizados mediante métodos ópticos sobre modelos a escala aumentada, sirvieron para validar el código numérico. Los resultados indicaron que la teoría irrotacional sobreestima significativamente el valor del ángulo del cono del spray generado, mientras que predice valores más bajos del espesor de la lámina líquida a la salida.

En trabajos posteriores, Sakman et al. (2000) y Xue et al. (2004) exploraron numéricamente la dependencia en las características del flujo de los parámetros geométricos no tenidos en cuenta por la teoría irrotacional.

Sakman et al. (2000) realizaron un estudio numérico del efecto de ciertos parámetros geométricos del atomizador en las características del flujo mediante un modelo axilsimétrico basado en el mismo método numérico que Jeng, Jog \& Benjamin (1998), y validado mediante datos experimentales. Los parámetros geométricos que estudiaron fueron las relaciones entre la longitud y el diámetro de la cámara, entre la longitud y el diámetro del orificio de salida y entre los diámetros de la cámara y del orificio de salida. Obtuvieron que un aumento de la relación entre la longitud y el diámetro de la cámara se traduce en un aumento en el espesor de la lámina líquida en el orificio de salida, una disminución del ángulo del cono del spray generado y de las componentes axial y azimutal de la velocidad del líquido a la salida, y una ligera disminución seguida de un aumento del coeficiente de descarga. Un aumento en la relación entre la longitud y el diámetro del orificio de salida da lugar a una disminución del espesor de la lámina líquida, del ángulo del cono del spray, del coeficiente de descarga, y de las componentes axial y azimutal de la velocidad del líquido 
a la salida. Finalmente, un aumento de la relación entre los diámetros de la cámara y del orificio de salida origina una disminución del espesor de la lámina líquida y del coeficiente de descarga, un aumento de las componentes axial y azimutal de la velocidad del líquido a la salida, y un aumento del ángulo del cono del spray seguido de una disminución del mismo.

El modelo numérico desarrollado por Xue et al. (2004) también estaba basado en el mismo método numérico empleado por Jeng, Jog \& Benjamin (1998), y fue también validado mediante datos experimentales. El modelo del atomizador estudiado constaba de unos canales de entrada cuyos ejes formaban un ángulo variable con el eje de la cámara, así como una sección divergente al final del orificio de salida, la cual se emplea para limitar el ángulo del cono del spray generado. Se estudió la influencia de cuatro parámetros geométricos: el ángulo de los canales de entrada respecto al eje de la cámara, el ángulo de la sección convergente anterior al orificio de salida, el ángulo de la sección divergente posterior al mismo, y la relación entre la longitud de dicha sección divergente y el diámetro del orificio de salida. Los resultados muestran que un incremento del ángulo de los canales de entrada respecto al eje de la cámara resulta en una disminución del espesor de la lámina líquida a la salida y del coeficiente de descarga, y un aumento del ángulo del cono del spray generado. Un incremento en el ángulo de la sección convergente se traduce en un incremento del espesor de la lámina líquida y del coeficiente de descarga, y una disminución del ángulo del cono del spray. El ángulo de la sección divergente tiene poca influencia en el coeficiente de descarga, mientras que al incrementarlo aumenta el ángulo del cono del spray y disminuye el espesor de la lámina líquida. Y tanto el ángulo del cono del spray como el espesor de la lámina líquida disminuyen cuando se aumenta la longitud de la sección divergente, sin que se vea afectado el coeficiente de descarga.

El efecto ejercido por varios parámetros geométricos y por el número de Reynolds en el núcleo de aire ha sido también investigado por Halder, Dash \& Som (2003) y NouriBorujerdi \& Kebriaee (2012), mientras que Hansen et al. (2002), Maatje, von Lavante \& Albina (2002) y von Lavante, Maatje \& Albina (2002) han llevado a cabo cálculos tridimensionales y de flujo no estacionario.

En los estudios numéricos y experimentales realizados por Halder, Dash \& Som (2003) sobre el núcleo de aire que se origina en el interior de un atomizador, se observó el aumento del diámetro del mismo en el orificio de salida debido al aumento de la velocidad axial en dicha región. Estos autores analizaron al efecto del número de Reynolds, concluyendo que para valores bajos del mismo, al incrementarlo aumenta el diámetro del núcleo de aire, debido al aumento de la velocidad azimutal. Sin embargo, para números de Reynolds 
elevados, la variación del mismo no tiene influencia en el diámetro del núcleo de aire, pues el efecto del incremento de la velocidad azimutal es contrarrestado por el efecto adverso de la resistencia de fricción. Además, observaron que el diámetro del núcleo de aire aumenta cuando lo hacen la relación entre los diámetros del orificio de salida y de la cámara o el ángulo de la sección convergente, o cuando disminuyen las relaciones entre los diámetros de los canales de entrada y de la cámara o entre la longitud del orificio y el diámetro de la cámara.

Nouri-Borujerdi \& Kebriaee (2012) estudiaron numéricamente el flujo en el interior del atomizador mediante simulaciones de flujo laminar y turbulento, y las compararon con resultados experimentales. Los resultados mostraron que las simulaciones realizadas con flujo laminar representan mejor el comportamiento del flujo en el atomizador que las realizadas con flujo turbulento, posiblemente debido a una sobreestimación de la viscosidad turbulenta.

Hansen et al. (2002) llevaron a cabo estudios numéricos y experimentales del flujo interno en un atomizador. El estudio numérico lo realizaron sobre un modelo tridimensional multifásico empleando el código comercial CFX-4.3, mientras que los ensayos experimentales los realizaron midiendo las velocidades mediante técnicas de anemometría Laser Doppler (LDA, Laser Doppler Anemometry). Las simulaciones mostraron la estructura de torbellino del flujo interno, con la formación del núcleo de aire, y la presencia de regiones de recirculación a la entrada de la sección convergente anterior al orificio de salida.

Maatje, von Lavante \& Albina (2002) y von Lavante, Maatje \& Albina (2002) estudiaron numéricamente el flujo en un atomizador mediante modelos bidimensionales y tridimensionales. El estudio lo realizaron empleando los códigos comerciales FLUENT y COMET, usando un algoritmo de captura de la entrefase del tipo VOF (Volume Of Fluid). Las simulaciones mostraron que las regiones de mayor velocidad axial del líquido se encontraban cerca del núcleo de aire y en la pared, pasando aproximadamente la mitad del líquido a través de estas zonas de la cámara a pesar de su reducida área. También mostraron la formación de torbellinos de Görtler en la pared cóncava de la cámara, y ondas de pequeña amplitud en la superficie del núcleo de aire que se originan en la pared opuesta al orificio de salida y viajan aguas abajo hacia el mismo. 


\section{Capítulo 2}

\section{Aproximación de flujo no viscoso irrotacional}

En el presente capítulo se estudiará el flujo en un atomizador de presión con giro considerando que el fluido de operación es no viscoso. Se comenzará formulando el problema. Veremos que el flujo se puede considerar prácticamente irrotacional y definiremos dos parámetros relevantes: el coeficiente de descarga y la constante del atomizador. Ésta última es un parámetro adimensional en el que se apoyan muchos de los estudios realizados previamente sobre flujo no viscoso, en los que se emplea la hipótesis adicional de flujo cuasi-unidireccional (Abramovich 1944, Novikov 1948, Taylor 1948, Giffen \& Muraszew 1953, Nieuwkamp 1985, Bayvel \& Orzechowski 1993). Estos estudios, de los que se incluirá un breve resumen, concluyen que las características del flujo en un atomizador dependen exclusivamente de la constante del atomizador. Para estudiar el efecto de otros parámetros geométricos se calculará numéricamente la solución del problema del flujo irrotacional sin ninguna hipótesis adicional, empleando el método de los elementos de contorno para resolver la ecuación de Laplace. Se estudiará también el fenómeno no estacionario de la propagación de ondas en la superficie del núcleo de aire que se genera en el interior del atomizador, y cuya formación ha sido observada en experimentos y simulaciones numéricas (Donjat et al. 2003, Yule \& Chinn 2000, Maatje, von Lavante \& Albina 2002, von Lavante, Maatje \& Albina 2002).

\subsection{Formulación del problema}

El flujo que se produce en el interior de un atomizador es debido a la diferencia de presión que existe a través del mismo, desde los canales de entrada al orificio de salida. 
Si estos canales de entrada son perpendiculares a un plano meridiano, entonces el flujo producido presenta inicialmente una componente azimutal de la velocidad únicamente, y las componentes axial y radial de la velocidad se generan debido al llenado de la cámara, sin ser impuestas por los canales de entrada. Sin embargo, si las direcciones de los canales de entrada forman un ángulo distinto de $90^{0}$ con un plano meridiano, entonces el flujo a la entrada presenta unas componentes axial y/o radial de velocidad, además de la componente azimutal.

Para un atomizador sencillo, con los canales de entrada perpendiculares a un plano meridiano, la velocidad axial que se establece en la cámara es debida a la conservación de la masa del fluido. El movimiento de giro impuesto al líquido es resultado de la posición tangencial de los canales de entrada, y se mantiene a través de la cámara y del orificio de salida. Idealmente, para líquidos poco viscosos, el número de Reynolds es grande comparado con la unidad, $R e \gg 1$, por lo que las fuerzas viscosas desempeñan un papel despreciable en la ecuación del movimiento en casi todo el campo fluido. Las fuerzas viscosas son importantes en ciertas capas delgadas, pero la presencia de estas capas no modifica drásticamente el flujo en la cámara del atomizador. Debido a que las fuerzas de viscosidad son despreciables en la mayor parte de la cámara, y a que las fuerzas de presión por unidad de masa derivan de un potencial, pues estamos considerando que el fluido es incompresible, la aceleración deriva de un potencial. Debido a ello, la circulación de la aceleración a lo largo de cualquier línea fluida cerrada es nula y la circulación de la velocidad se mantiene constante, y los tubos de vorticidad, que son superficies fluidas, mantienen su circulación en su evolución. Si además el movimiento es irrotacional a la entrada del atomizador, se mantendrá irrotacional. Así pues, este movimiento de giro se puede considerar prácticamente irrotacional, es decir, $\tilde{\omega}=\nabla \times \tilde{\mathbf{v}}=0$, y por tanto $\tilde{\mathbf{v}}=\nabla \phi$, donde $\vec{v}$ es la velocidad del líquido y $\phi$ es el potencial de velocidad.

Además el flujo se puede considerar axilsimétrico en la mayor parte de la cámara del atomizador. En coordenadas cilíndricas $(x, r, \vartheta)$, donde $x$ es la distancia a lo largo del eje de la cámara y $r$ es la distancia al eje, y llamando $(u, v, w)$ a las correspondientes componentes de la velocidad,

$$
\nabla \times \tilde{\mathbf{v}}=\left(\frac{1}{r} \frac{\partial(r w)}{\partial r},-\frac{\partial w}{\partial x}, \frac{\partial v}{\partial x}-\frac{\partial u}{\partial r}\right)=(0,0,0)
$$

De la segunda componente de la expresión para la vorticidad (2.1) se observa que la velocidad azimutal $w$ es independiente de la coordenada axial $x$. Teniendo en cuenta esto, de la primera componente de (2.1) se obtiene:

$$
w r=\frac{\Gamma}{2 \pi}
$$


donde $\Gamma$ es la circulación sobre cualquier curva cerrada simple que rodee al eje de la cámara. Así pues, la componente azimutal de la velocidad es $w=\Gamma /(2 \pi r)$, que representa un torbellino potencial. La velocidad azimutal es menor cerca de la pared de la cámara y mayor en las proximidades del eje de la misma. Dado que la velocidad azimutal crece sin límite al aproximarse al eje de la cámara, la presión debe disminuir, como se verá posteriormente en la ecuación de Bernoulli. Como la cámara está abierta, y en su exterior hay un gas a una cierta presión $p_{a}$, esta disminución de la presión hace que el gas entre en la cámara durante el funcionamiento del atomizador, ocupando una cierta región en torno al eje, llamada núcleo de aire, donde, despreciando las variaciones de presión debidas al movimiento del gas, la presión tiene el valor uniforme $p_{a}$. El radio del núcleo de aire debe calcularse como parte de la solución. La presencia del núcleo de aire asegura que el líquido en el orificio de salida forme una lámina anular. Una partícula fluida describirá una trayectoria helicoidal en su paso a través de dicho orificio de salida.

Llamamos $R_{w}(x)$ al radio de la pared interna del atomizador, que toma los valores $R_{s}$ y $R_{0}$ en la cámara y en el orificio de salida, respectivamente, $r_{a c}(x)$ al radio del núcleo de aire, cuyos valores en el interior de la cámara y en el orificio de salida son $r_{s}$ y $r_{0}, R_{i}$ a la distancia del eje de los canales de entrada al eje de la cámara, $L_{s}$ y $L_{0}$ a las longitudes de la cámara y del orificio de salida, respectivamente, y $\alpha$ al ángulo de la pared convergente. Un esquema se muestra en la figura 2.1.

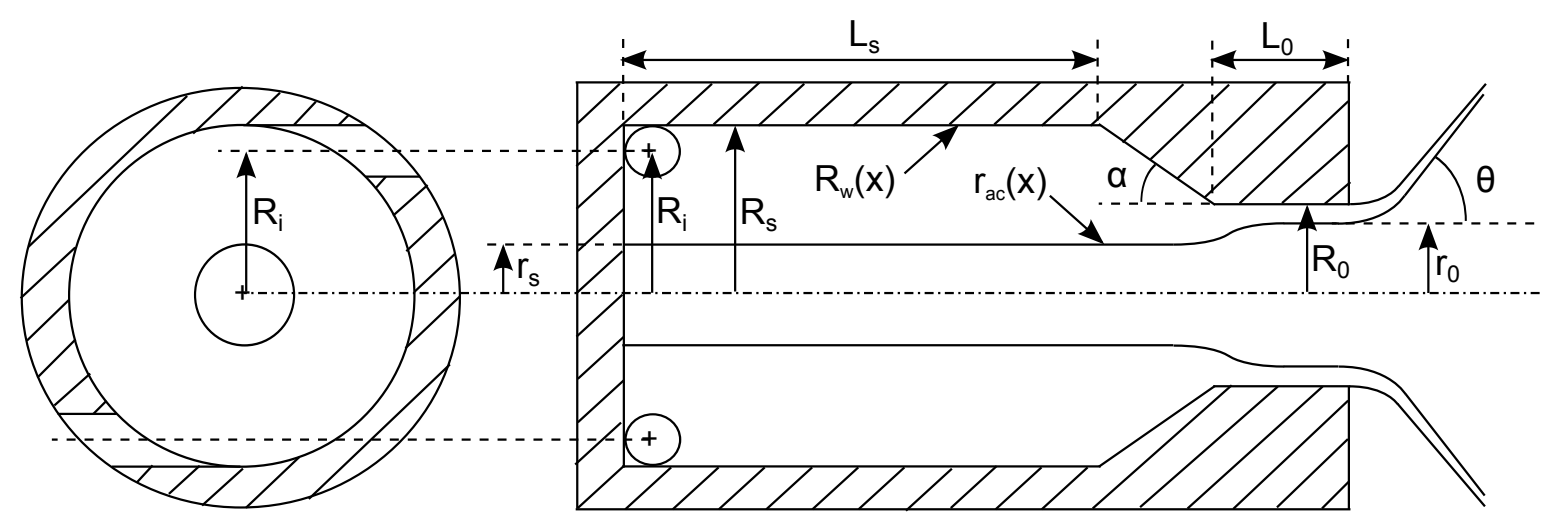

Figura 2.1 - Esquema de un atomizador de presión con giro con 2 canales de entrada.

El flujo real no será axilsimétrico cerca de los canales de entrada a la cámara. Sin embargo, si (2.2) se extiende hasta estos canales, usando valores efectivos de su área 
transversal total, $A_{i}$, y de su distancia al eje de la cámara, $R_{i}$, se obtiene:

$$
w_{i}=\frac{Q}{A_{i}} \quad \text { y } \quad \frac{\Gamma}{2 \pi}=w_{i} R_{i}
$$

donde $Q$ es el caudal de líquido que se introduce en la cámara y $w_{i}$ debe entenderse como un valor efectivo de la velocidad azimutal de entrada, que se obtiene donde el flujo puede empezar a considerarse axilsimétrico. De esta forma, la velocidad azimutal en cualquier punto del líquido viene dada por la siguiente expresión:

$$
w=\frac{Q}{A_{i}} \frac{R_{i}}{r}
$$

Usando estos resultados puede escribirse

$$
\phi=\frac{\Gamma}{2 \pi} \vartheta+\varphi(x, r)
$$

con:

$$
u=\frac{\partial \varphi}{\partial x} \quad \mathrm{y} \quad v=\frac{\partial \varphi}{\partial r}
$$

La ecuación de continuidad $\nabla \cdot \tilde{\mathbf{v}}=0$ se reduce a la ecuación de Laplace:

$$
\nabla^{2} \varphi=\frac{\partial^{2} \varphi}{\partial x^{2}}+\frac{1}{r} \frac{\partial}{\partial r}\left(r \frac{\partial \varphi}{\partial r}\right)=0
$$

mientras que la ecuación de cantidad de movimiento se reduce a la ecuación de EulerBernoulli:

$$
\frac{\partial \varphi}{\partial t}+\frac{1}{2}|\nabla \varphi|^{2}+\frac{\Gamma^{2}}{8 \pi^{2} r^{2}}+\frac{p}{\rho}=C(t)
$$

donde $C(t)$ es una función del tiempo y se han despreciado las fuerzas másicas. Para un flujo estacionario se obtiene la ecuación de Bernoulli:

$$
\frac{1}{2}|\nabla \varphi|^{2}+\frac{\Gamma^{2}}{8 \pi^{2} r^{2}}+\frac{p}{\rho}=\frac{p_{0}}{\rho}
$$

donde $p_{0}$ es la presión de remanso del líquido aportado a la cámara.

La ecuación de Bernoulli particularizada en la superficie del núcleo de aire es:

$$
\frac{1}{2}\left|\nabla \varphi_{a c}\right|^{2}+\frac{\Gamma^{2}}{8 \pi^{2} r_{a c}^{2}}=\frac{p_{0}-p_{a}}{\rho}
$$

donde el subíndice $a c$ indica condiciones en la superficie.

Cuando el líquido entra en la parte convergente de la cámara la componente axial de la velocidad aumenta, mientras que la velocidad azimutal es la misma para un radio dado. Como la velocidad total aumenta, debido al incremento de la componente axial, la 
presión del líquido debe disminuir para un radio dado. Por otra parte, como la presión es constante en la superficie del núcleo de aire, (2.7) muestra que el aumento de la velocidad está asociado a un aumento del radio del núcleo de aire.

La lámina de líquido que alcanza el orificio de salida comienza a diverger radialmente aguas abajo del mismo, donde ya no está confinada por las paredes de la cámara, y acaba rompiéndose en ligamentos y posteriormente en gotas a cierta distancia de la cámara. $\mathrm{Si}$ esta distancia es suficientemente grande, la lámina, que es estacionaria hasta poco antes del punto de rotura, toma una forma cónica. La presión del líquido en la lámina tiende rápidamente a $p_{a}$, la componente azimutal de la velocidad tiende a cero al crecer el radio de la lámina, y la velocidad del líquido tiende al valor $U_{\infty}=\sqrt{2\left(p_{0}-p_{a}\right) / \rho}$, dado por la ecuación de Bernoulli (2.6). El espesor de la lámina cónica, obtenido de la ecuación de continuidad, es $h(x)=Q /\left(2 \pi U_{\infty} x \tan \theta\right)$, donde $\theta$ es el semiángulo del cono, que es desconocido. Todo esto es válido para el caso en que se desprecie la tensión superficial $\sigma$. Sin embargo, el efecto de la tensión superficial sí se tendrá en cuenta en capítulos posteriores.

Sea $F(x, r, t)=0$ la ecuación de la superficie del líquido, con $F<0$ en el líquido. Esta superficie es desconocida e incluye la superficie del núcleo de aire en el interior de la cámara y las dos caras de la lámina líquida en el exterior. Para calcular $F(x, r, t)$ y el potencial de velocidades $\varphi(x, r, t)$ debe resolverse el siguiente problema de frontera libre

$$
\nabla^{2} \varphi=0
$$

en el líquido, $F<0$, con las condiciones de contorno

$$
\frac{D F}{D t}=0 \quad \text { y } \quad \frac{D \varphi}{D t}=\frac{1}{2}|\nabla \varphi|^{2}-\frac{\Gamma^{2}}{8 \pi^{2} r^{2}}-\frac{p_{a}}{\rho}+C
$$

en la superficie libre $F=0$, donde $D(\bullet) / D t=\partial(\bullet) / \partial t+\nabla \varphi \cdot \nabla(\bullet)$,

$$
\mathbf{n} \cdot \nabla \varphi=0
$$

en las paredes internas de la cámara, siendo $\mathbf{n}$ la normal exterior al líquido, y

$$
\mathbf{n} \cdot \nabla \varphi=-\frac{Q}{A_{i}}
$$

en las secciones efectivas de los canales de entrada.

La primera ecuación (2.9) expresa la condición de que la superficie del líquido es una superficie fluida, y la segunda se obtiene particularizando la ecuación de Euler-Bernoulli (2.5) en la superficie del líquido, donde $p=p_{a}$. La función desconocida $C(t)$ puede hacerse cero redefiniendo $\varphi$, o bien determinarse con la condición adicional de que $\varphi$ tome un valor específico en algún punto del líquido, por ejemplo, $\varphi=0$ en la línea de contacto de la superficie libre con la pared de la cámara opuesta al orificio de salida. 


\section{El coeficiente de descarga}

El coeficiente de descarga $c_{d}$ es un parámetro adimensional que se define como la relación entre el caudal real y el correspondiente a un flujo en el que el líquido emerja del orificio como un chorro sin núcleo de aire y sin giro, por lo que el flujo tendrá sólo componente axial, $u=U_{\infty}$ :

$$
c_{d}=\frac{Q}{\pi R_{0}^{2} U_{\infty}}
$$

donde $U_{\infty}=\sqrt{2\left(p_{0}-p_{a}\right) / \rho}$. Así pues, se obtiene la siguiente expresión para el coeficiente de descarga:

$$
c_{d}=\frac{Q}{\pi R_{0}^{2} \sqrt{\frac{2\left(p_{0}-p_{a}\right)}{\rho}}}
$$

También se puede expresar $c_{d}$ de otra forma teniendo en cuenta que $U_{\infty}=\Gamma /\left(2 \pi r_{s}\right)$, donde $r_{s}$ es el radio del núcleo de aire en la pared de la cámara opuesta al orificio de salida:

$$
c_{d}=\frac{2 \pi Q}{\Gamma R_{0}} \frac{r_{s} / R_{0}}{\pi}
$$

donde $(2 \pi Q) /\left(\Gamma R_{0}\right)$ es el caudal adimensional, luego el coeficiente de descarga $c_{d}$ es directamente proporcional al caudal adimensional y al radio del núcleo de aire en la cámara, $r_{s}$, adimensionalizado con el radio del orificio de salida, $R_{0}$.

\section{La constante del atomizador}

Muchos de los estudios realizados sobre el flujo no viscoso en atomizadores de presión con giro se apoyan en un parámetro adimensional conocido como la constante del atomizador. Este parámetro ha sido definido de diversas formas por los diferentes autores. En este estudio se ha considerado la siguiente:

$$
\Delta=\frac{A_{i}}{4 R_{i} R_{0}}
$$

De la expresión (2.3) puede expresarse el área total de los canales de entrada en función del caudal, $A_{i}=Q / w_{i}$. La velocidad azimutal a la entrada es $w_{i}=\Gamma /\left(2 \pi R_{i}\right)$, por lo que el área de entrada en función del caudal es:

$$
A_{i}=\frac{2 \pi Q R_{i}}{\Gamma}
$$

Sustituyendo (2.16) en (2.15) se obtiene una expresión para la constante del atomizador en función del caudal:

$$
\Delta=\frac{1}{4} \frac{2 \pi Q}{\Gamma R_{0}}=\frac{Q}{\pi R_{0}^{2} U_{\infty}} \frac{\pi^{2}}{2} \frac{U_{\infty}}{\Gamma / R_{0}}
$$

Esto es, la constante del atomizador es igual a la cuarta parte del caudal adimensional. 


\subsection{El flujo sobre un rebosadero y a la salida de la cámara de un atomizador: la condición de caudal máximo}

Muchos de los estudios del flujo irrotacional en un atomizador de presión con giro consideran condiciones críticas en el orificio de salida, en el sentido de que la velocidad ha de ser la misma que la de propagación de ondas en la superficie del líquido, de forma que las mismas no se puedan propagar aguas arriba. Como veremos, esta condición implica que la velocidad y el espesor de la lámina líquida en el orificio de salida se ajustan de forma que el caudal es mayor de lo que sería para cualquier otro valor de la velocidad de salida del líquido. Esta idea surge del comportamiento del flujo sobre un rebosadero.

Un rebosadero es básicamente un obstáculo sumergido perpendicular a la corriente de un río o un canal, que se usa para medir el caudal de la corriente. El agua se remansa delante del rebosadero, aumentando su nivel, fluye sobre el mismo y se vierte aguas abajo. El caudal sobre la cima del rebosadero es el mismo que fluye aguas arriba y aguas abajo del mismo. Sin embargo, el flujo es más rápido sobre el rebosadero debido al menor área de la sección transversal del volumen de agua. El nivel de la superficie del agua sobre el rebosadero es menor que el nivel aguas arriba. En un rebosadero cuya altura varíe suavemente en la dirección de la corriente, este nivel, y la velocidad del agua, se ajustan de manera que el flujo es crítico en el punto más alto del rebosadero y el caudal es mayor que lo que sería de no cumplirse esta condición.

En un atomizador la cámara es análoga a la región aguas arriba del rebosadero, y el orificio de salida se corresponde con el rebosadero. El diámetro del núcleo de aire cambia entre la cámara y el orificio de salida, ajustándose en esta última región, además de la velocidad axial, de forma que el flujo sea crítico.

\subsubsection{El flujo sobre un rebosadero}

En el flujo sobre un rebosadero la forma de la superficie y el movimiento del líquido dependen de la fuerza de gravedad. En el flujo en un atomizador de presión con giro la forma de la superficie y el movimiento del líquido dependen de la fuerza centrífuga. A continuación se detalla la condición de caudal máximo aplicada al flujo sobre un rebosadero.

Consideremos el flujo en un canal de anchura B con un rebosadero, el cual es un obstáculo de altura $H(x)$ sobre el fondo del canal. La profundidad del líquido, entre su 
superficie libre y el fondo del canal o la parte superior del rebosadero, es $h(x)$, y su velocidad es $u(x)$, siendo dichas magnitudes constantes, de valores $h_{1}$ y $u_{1}$ respectivamente, aguas arriba del rebosadero. Un esquema del mismo se puede observar en la figura 2.2:

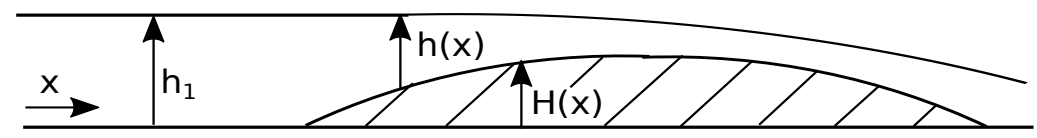

Figura 2.2 - Flujo, de izquierda a derecha, de un líquido sobre un rebosadero.

La ecuación de Bernoulli para este flujo, aplicada en la superficie libre del líquido sobre el rebosadero, es la siguiente:

$$
\rho g(h+H)+\frac{1}{2} \rho u^{2}=\rho g h_{1}+\frac{1}{2} \rho u_{1}^{2}
$$

Si $h(x)$ es pequeña frente a la longitud del obstáculo sumergido, la velocidad $u$ se puede considerar uniforme en cada sección $x$ constante. El caudal viene dado por:

$$
Q=u h B
$$

Derivando con respecto a $x$ la ecuación (2.18):

$$
\frac{\mathrm{d} h}{\mathrm{~d} x}+\frac{\mathrm{d} H}{\mathrm{~d} x}+\frac{u}{g} \frac{\mathrm{d} u}{\mathrm{~d} x}=0
$$

Haciendo lo mismo con la ecuación (2.19), y teniendo en cuenta que $\mathrm{d} Q / \mathrm{d} x=0$ :

$$
u \frac{\mathrm{d} h}{\mathrm{~d} x}+h \frac{\mathrm{d} u}{\mathrm{~d} x}=0
$$

Eliminando $\mathrm{d} h / \mathrm{d} x$ entre las expresiones (2.20) y (2.21) se obtiene:

$$
\left(u^{2}-g h\right) \frac{\mathrm{d} u}{\mathrm{~d} x}=0
$$

en el punto más alto del obstáculo sumergido, donde $\mathrm{d} H / \mathrm{d} x=0$. Para que la anterior expresión se cumpla la velocidad sobre el rebosadero ha de ser igual a un valor $u_{c}$ definido como:

$$
u_{c}=\sqrt{g h}
$$

que coincide con la velocidad de propagación de las ondas gravitatorias respecto al líquido. 
Por otra parte, (2.18) y (2.19) pueden interpretarse como dos ecuaciones que determinan $u$ y $Q$ como funciones de la profundidad $h$ en el punto más alto del rebosadero. Derivando (2.19) respecto a $h$ :

$$
\frac{\partial Q}{\partial h}=B u+B h \frac{\partial u}{\partial h}
$$

Haciendo lo mismo con (2.18):

$$
g+u \frac{\partial u}{\partial h}=0
$$

Despejando $\partial u / \partial h$ de (2.25) y sustituyendo en (2.24):

$$
\frac{\partial Q}{\partial h}=\frac{B}{u}\left(u^{2}-g h\right)
$$

Teniendo en cuenta que la velocidad en el punto más alto del rebosadero ha de ser $u_{c}$, como hemos visto anteriormente, se llega finalmente a:

$$
\frac{\partial Q}{\partial h}=0
$$

Así pues, la altura y velocidad del líquido sobre el rebosadero se ajustarán de forma que el caudal $Q$ sea máximo, en el sentido de la derivación anterior.

\subsubsection{El flujo a la salida de la cámara de un atomizador}

Si el espesor de la lámina líquida en el orificio de salida es mucho menor que la longitud de dicho orificio, se puede considerar que la velocidad axial, $u_{0}$, es constante en una sección transversal, $\partial u_{0} / \partial r=0$, y la velocidad radial, $v_{0}$, es despreciable frente a las velocidades axial y azimutal, $u_{0}$ y $w_{0}$. El caudal $Q$ puede expresarse:

$$
Q=u_{0} \pi\left(R_{0}^{2}-r_{0}^{2}\right)
$$

Teniendo en cuenta esto, la ecuación de Bernoulli (2.6) aplicada en la superficie del núcleo de aire en el orificio de salida se reduce a:

$$
\frac{1}{2} u_{0}^{2}+\frac{\Gamma^{2}}{8 \pi^{2} r_{0}^{2}}=\frac{p_{0}-p_{a}}{\rho}
$$

De (2.29) se llega a la siguiente expresión para la velocidad axial $u_{0}$ :

$$
u_{0}=\sqrt{\frac{2\left(p_{0}-p_{a}\right)}{\rho}-\frac{\Gamma^{2}}{4 \pi^{2} r_{0}^{2}}}
$$


Derivando la expresión (2.28) respecto al radio del núcleo de aire en el orificio de salida, $r_{0}$ :

$$
\frac{\partial Q}{\partial r_{0}}=\pi\left(R_{0}^{2}-r_{0}^{2}\right) \frac{\partial u_{0}}{\partial r_{0}}-2 u_{0} \pi r_{0}
$$

Derivando respecto a $r_{0}$ la velocidad axial $u$ definida en (2.30):

$$
\frac{\partial u_{0}}{\partial r_{0}}=\frac{1}{u_{0}} \frac{\Gamma^{2}}{4 \pi^{2} r_{0}^{3}}
$$

Sustituyendo (2.32) en (2.31):

$$
\frac{\partial Q}{\partial r_{0}}=\frac{2 \pi r_{0}}{u_{0}}\left(\frac{\Gamma^{2}\left(R_{0}^{2}-r_{0}^{2}\right)}{8 \pi^{2} r_{0}^{4}}-u_{0}^{2}\right)
$$

Así pues, el caudal será máximo, en el sentido de que $\partial Q / \partial r_{0}=0$, cuando la velocidad $u_{0}$ tome un valor $u_{c}$ definido como:

$$
u_{c}^{2}=\frac{\Gamma^{2}\left(R_{0}^{2}-r_{0}^{2}\right)}{8 \pi^{2} r_{0}^{4}}
$$

La anterior expresión se puede escribir como:

$$
\frac{u_{c}}{\Gamma /\left(2 \pi R_{0}\right)}=\frac{1}{\left(r_{0} / R_{0}\right)^{2}} \sqrt{\frac{1-\left(r_{0} / R_{0}\right)^{2}}{2}}
$$

que, como veremos en la Sección 2.5, coincide con la velocidad de propagación de las ondas en el orificio de salida en el límite $\left(k r_{0}, k R_{0}\right) \ll 1$, donde $k$ es el número de onda.

\subsection{Revisión de la aproximación cuasi-unidireccional}

La aproximación del problema mediante modelos irrotacionales ha permitido obtener mucha información sobre las principales características del flujo. Muchos de estos modelos se basan en la aproximación de flujo cuasi-unidireccional, complementada con la condición de que el flujo es crítico a la salida, en el sentido en que su velocidad axial es igual que la de propagación de ondas, y que en el orificio de salida la velocidad no tiene componente radial, por lo que, de la tercera componente de la expresión de la vorticidad (2.1) se obtiene $\partial u / \partial r=0$, es decir, que la velocidad axial es uniforme en cada sección transversal. Para este flujo crítico el diámetro del núcleo de aire y la velocidad a la salida se ajustan de forma que el caudal sea máximo. Esta condición deriva de la analogía entre el flujo en un atomizador de presión con giro y el flujo sobre un rebosadero, que se explicó más arriba. La teoría cuasi-unidireccional predice que los resultados dependen únicamente de la constante del atomizador, y son independientes del resto de parámetros geométricos. 


\subsubsection{Coeficiente de descarga y espesor de la lámina}

Para calcular las características del flujo en el orificio, Taylor (1948) aplicó la ecuación de Bernoulli en la superficie libre del líquido en el orificio de salida, donde la presión es la atmosférica:

$$
U_{\infty}^{2}=u_{0}^{2}+\left(\frac{\Gamma}{2 \pi r_{0}}\right)^{2}
$$

donde $U_{\infty}=\sqrt{2\left(p_{0}-p_{a}\right) / \rho}$. En la superficie libre del líquido en el interior de la cámara, en la pared opuesta al orificio de salida, la velocidad axial es nula, $u_{s}=0$, luego el radio del núcleo de aire en el interior de la cámara es $r_{s}=\Gamma /\left(2 \pi U_{\infty}\right)$. Si el radio del núcleo de aire en el orificio tuviese el mismo valor, como aseguraban autores anteriores a Taylor erroneamente, entonces, de la ecuación de Bernoulli se derivaría que la velocidad axial en el orificio sería nula, es decir, no habría flujo a través del orificio. Taylor adimensionalizó la ecuación de Bernoulli (2.36) usando las variables $x=u_{0} / U_{\infty}, y=\Gamma /\left(2 \pi R_{0} U_{\infty}\right)=r_{s} / R_{0}$, $z=r_{0} / R_{0}$, es decir:

$$
1=x^{2}+\frac{y^{2}}{z^{2}}
$$

Se puede expresar el caudal, dependiente de la velocidad de salida y del radio del núcleo de aire en el orificio, $Q=\pi u_{0}\left(R_{0}^{2}-r_{0}^{2}\right)$, en función de las anteriores variables:

$$
Q=\pi R_{0}^{2} U_{\infty}\left(1-z^{2}\right) x=\pi R_{0}^{2} U_{\infty}\left(1-z^{2}\right) \sqrt{1-\frac{y^{2}}{z^{2}}}
$$

y el coeficiente de descarga, $c_{d}=Q / \pi R_{0}^{2} U_{\infty}$ :

$$
c_{d}=\left(1-z^{2}\right) \sqrt{1-\frac{y^{2}}{z^{2}}}
$$

La constante del atomizador se puede expresar en función de las variables $(x, y, z)$ introduciendo la expresión para el caudal (2.38) en (2.17):

$$
\Delta=\frac{\pi}{4} \frac{1-z^{2}}{y} \sqrt{1-\frac{y^{2}}{z^{2}}}
$$

Si se fija la variable $y$, se necesitaría una ecuación más para determinar el problema. Taylor emplea la condición adicional de que el flujo en la salida ha de ser crítico, es decir, el caudal ha de satisfacer $\partial Q / \partial z=0$, de donde se obtiene el valor del radio del núcleo de aire en el orificio en función de su valor en el interior de la cámara:

$$
z^{2}=\frac{y^{2}}{4}+\sqrt{\frac{y^{4}}{16}+\frac{y^{2}}{2}}
$$


Despejando $y^{2}$ de $(2.41)$ :

$$
y^{2}=\frac{2 z^{4}}{1+z^{2}}
$$

Sustituyendo (2.42) en (2.39) se obtiene el coeficiente de descarga $c_{d}$ en función de $z$ :

$$
c_{d}=\left[\frac{\left(1-z^{2}\right)^{3}}{1+z^{2}}\right]^{1 / 2}
$$

y sustituyendo (2.42) en (2.40) se obtiene la constante del atomizador $\Delta$ en función de $z$ :

$$
\Delta=\frac{\pi}{4 \sqrt{2}} \frac{\left(1-z^{2}\right)^{3 / 2}}{z^{2}}
$$

El espesor adimensional de la lámina líquida en el orificio de salida, escalado con el radio de dicho orificio $R_{0}$, es:

$$
1-\frac{r_{0}}{R_{0}}=1-z
$$

Los resultados obtenidos por Taylor se muestran en la figura 2.3.

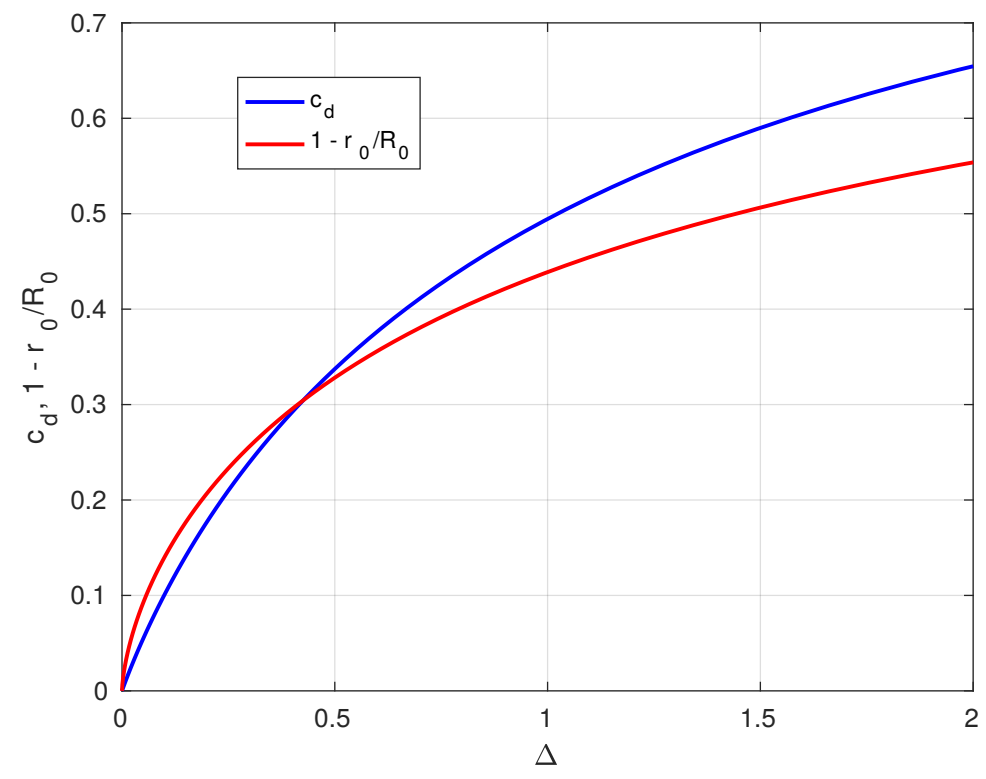

Figura 2.3 - Coeficiente de descarga $c_{d}$ (azul) y espesor adimensional de la lámina líquida en el orificio de salida $1-r_{0} / R_{0}$ (rojo) en función de la constante del atomizador $\Delta$ (Taylor 1948).

Giffen \& Muraszew (1953) propusieron una ecuación que relaciona el radio del núcleo de aire en el orificio de salida $\left(r_{0}\right)$, el coeficiente de descarga $\left(c_{d}\right)$ y una constante del atomizador modificada que definen como $\Delta_{G M}=A_{i} /\left(\pi R_{s} R_{0}\right)=4 R_{i} \Delta /\left(\pi R_{s}\right)$. Consideraron 
que la velocidad azimutal a la entrada, $w_{i}=Q / A_{i}$, se produce a un radio igual al de la cámara, $R_{s}$, luego $R_{i}=R_{s}$, y $\Delta_{G M}=(4 / \pi) \Delta$. Planteando la ecuación de Bernoulli en la superficie del núcleo de aire en el orificio de salida, donde la presión es la atmosférica:

$$
u_{0}^{2}+w_{0}^{2}=\frac{2\left(p_{0}-p_{a}\right)}{\rho}
$$

La expresión para la velocidad azimutal es:

$$
w=\frac{Q R_{s}}{A_{i}} \frac{1}{r}
$$

En concreto, en la superficie del núcleo de aire en el orificio de salida:

$$
w_{0}=\frac{Q R_{s}}{A_{i}} \frac{1}{r_{0}}
$$

Teniendo en cuenta que la velocidad axial es uniforme en una sección transversal en el orificio de salida, la misma se puede expresar de la siguiente forma:

$$
u_{0}=\frac{Q}{\pi\left(R_{0}^{2}-r_{0}^{2}\right)}
$$

Sustituyendo (2.48) y (2.49) en la ecuación de Bernoulli (2.46):

$$
\frac{Q^{2}}{\pi^{2}\left(R_{0}^{2}-r_{0}^{2}\right)^{2}}+\frac{Q^{2} R_{s}^{2}}{A_{i}^{2}} \frac{1}{r_{0}^{2}}=\frac{2\left(p_{0}-p_{a}\right)}{\rho}
$$

Introduciendo las expresiones para el coeficiente de descarga $c_{d},(2.13)$, la constante del atomizador modificada $\Delta_{G M}$, y considerando la variable $\zeta=r_{0}^{2} / R_{0}^{2}$, que es equivalente al cuadrado de la variable $z$ introducida por Taylor (1948), $\zeta=z^{2}$ :

$$
\frac{1}{c_{d}^{2}}=\frac{1}{(1-\zeta)^{2}}+\frac{1}{\Delta_{G M}^{2} \zeta}
$$

La ecuación (2.51) contiene dos incógnitas, $\zeta$ y $c_{d}$. Giffen \& Muraszew emplearon también la condición de que el caudal ha de ser máximo a la salida, luego el coeficiente de descarga $c_{d}$ expresado en función de $\zeta$ debe ser máximo, o $1 / c_{d}^{2}$ ha de ser mínimo. Derivando $\partial\left(1 / c_{d}^{2}\right) / \partial \zeta=0$ :

$$
\frac{2}{(1-\zeta)^{3}}-\frac{1}{\Delta_{G M}^{2} \zeta^{2}}=0
$$

de donde se obtiene:

$$
\Delta_{G M}=\left[\frac{(1-\zeta)^{3}}{2 \zeta^{2}}\right]^{1 / 2}
$$

Sustituyendo (2.53) en (2.51):

$$
c_{d}=\left[\frac{(1-\zeta)^{3}}{1+\zeta}\right]^{1 / 2}
$$


El espesor de la lámina líquida en el orificio de salida, adimensionalizado con el radio de dicho orificio $R_{0}$, es:

$$
1-\frac{r_{0}}{R_{0}}=1-\sqrt{\zeta}
$$

Los resultados obtenidos por Giffen \& Muraszew se muestran en la figura 2.4.

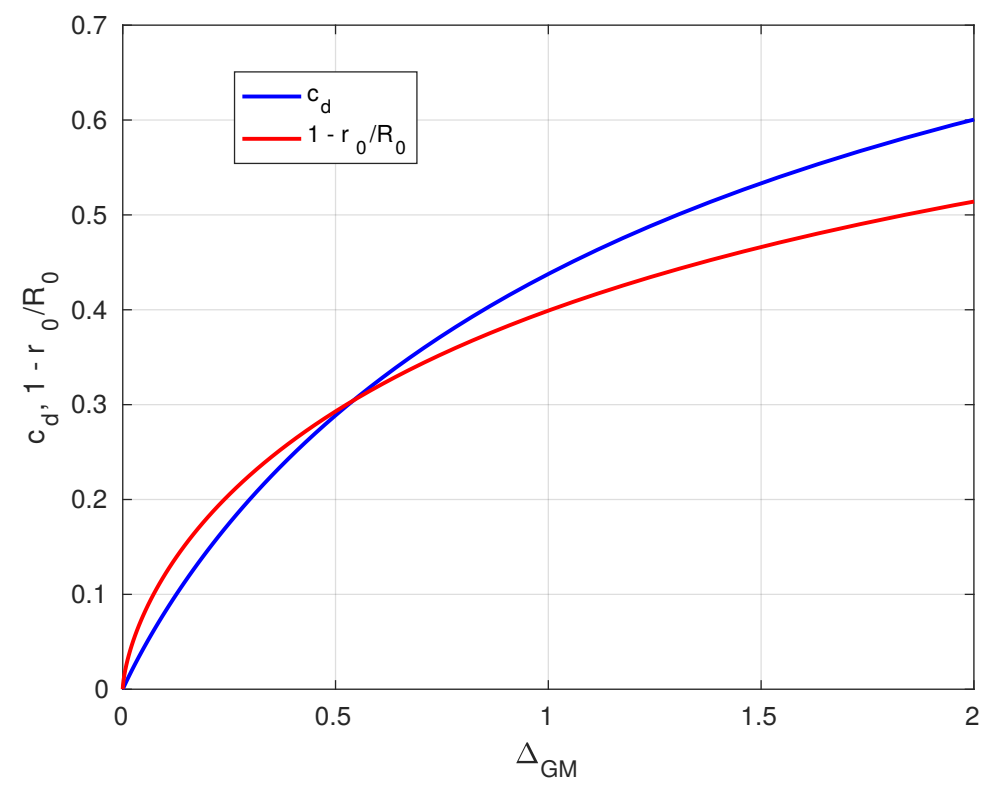

Figura 2.4 - Coeficiente de descarga $c_{d}$ (azul) y espesor adimensional de la lámina líquida en el orificio de salida $1-r_{0} / R_{0}$ (rojo) en función de la constante del atomizador $\Delta_{G M}$ (Giffen \& Muraszew 1953).

Nieuwkamp (1985) asumió en su estudio que en el atomizador la componente azimutal de la velocidad es mucho mayor que las otras, $v \ll w$ y $u \ll w$, de forma que la ecuación de cantidad de movimiento en dirección radial se reduce a:

$$
\frac{w^{2}}{r}=\frac{1}{\rho} \frac{\partial p}{\partial r}
$$

Introduciendo el valor de la velocidad azimutal correspondiente a la de un torbellino $w=\Gamma /(2 \pi r)$ en $(2.56)$, y refiriendo las presiones a la presión del gas exterior $\left(p_{a}=0\right.$ en las ecuaciones anteriores), se obtiene la siguiente expresión para la presión:

$$
p=\frac{\rho \Gamma^{2}}{8 \pi^{2}}\left(\frac{1}{r_{s}^{2}}-\frac{1}{r^{2}}\right)
$$

donde $r_{s}$ es el radio del núcleo de aire en el interior de la cámara. El caudal viene dado por $Q=A_{i} w_{i}$, donde Nieuwkamp consideró que la velocidad azimutal de entrada $w_{i}$ se 
produce a un radio correspondiente al que se encuentra el centro de los canales de entrada, luego $w_{0} r_{0}=w_{i} R_{i}$, por lo que la expresión para el caudal queda:

$$
Q=A_{i} \frac{w_{0} r_{0}}{R_{i}}
$$

Introduciendo (2.57) en la expresión (2.49) para la velocidad axial en la salida del orificio $u_{0}$ :

$$
u_{0}=\frac{A_{i} w_{0} r_{0}}{\pi\left(R_{0}^{2}-r_{0}^{2}\right) R_{i}}
$$

Para las condiciones de contorno en la salida, Nieuwkamp plantea una analogía con el flujo sobre un rebosadero. Sobre el punto más alto de un rebosadero, el líquido fluye a la velocidad de las ondas en la superficie, la cual depende de la gravedad y de la altura del líquido sobre el rebosadero de la forma $u^{*}=\sqrt{g h^{*}}$. Sustituyendo la gravedad $g$ por la aceleración centrífuga $w_{0}^{2} / r_{0}$ y la altura del líquido sobre el rebosadero $h^{*}$ por el espesor de la lámina líquida en el orificio de salida $R_{0}-r_{0}$ se obtiene una nueva expresión para la componente axial de la velocidad:

$$
u_{0}=\sqrt{\frac{w_{0}^{2}}{r_{0}}\left(R_{0}-r_{0}\right)}
$$

Combinando las ecuaciones (2.58) y (2.59) se obtiene la siguiente expresión para el radio del núcleo de aire en el orificio:

$$
\sqrt{\frac{R_{0}-r_{0}}{r_{0}}}\left(R_{0}^{2}-r_{0}^{2}\right) \frac{\pi R_{i}}{A_{i} r_{0}}-1=0
$$

Teniendo en cuenta la expresión (2.15), la relación (2.60) puede expresarse en función de la constante del atomizador, $\Delta$, y del radio del núcleo de aire a la salida adimensionalizado con el radio del orificio, $r_{0} / R_{0}$ :

$$
\Delta=\frac{\pi}{4} \frac{1-\left(r_{0} / R_{0}\right)^{2}}{r_{0} / R_{0}} \sqrt{\frac{1-r_{0} / R_{0}}{r_{0} / R_{0}}}
$$

Los resultados obtenidos por Nieuwkamp se muestran en la figura 2.5. 


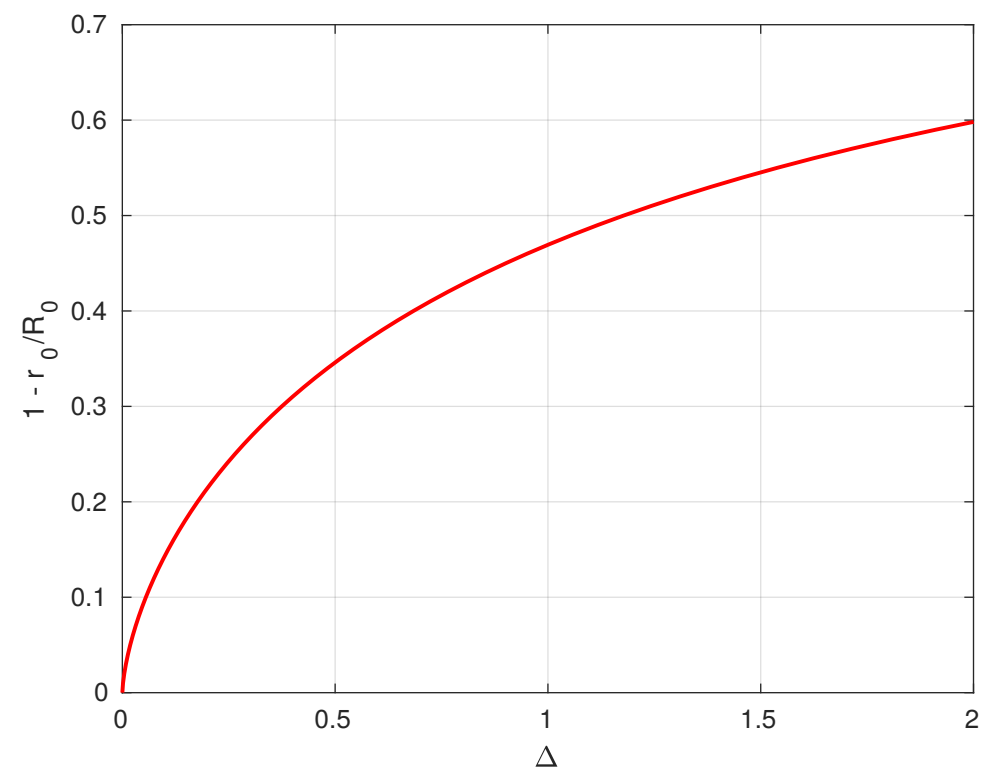

Figura 2.5 - Espesor adimensional de la lámina líquida en el orificio de salida $1-r_{0} / R_{0}$ en función de la constante del atomizador $\Delta$ (Nieuwkamp 1985).

Bayvel \& Orzechowski (1993) consideraron en su estudio que la velocidad azimutal a la entrada, $w_{i}=Q / A_{i}$, se produce a un radio correspondiente al que se encuentra el centro de los canales de entrada, $R_{i}$, luego la expresión para la velocidad azimutal es la anteriormente definida en (2.4). La ecuación de Bernoulli es entonces:

$$
\frac{Q^{2}}{\pi^{2}\left(R_{0}^{2}-r_{0}^{2}\right)^{2}}+\frac{Q^{2} R_{i}^{2}}{A_{i}^{2}} \frac{1}{r_{0}^{2}}=\frac{2\left(p_{0}-p_{a}\right)}{\rho}
$$

La constante del atomizador que emplearon la definieron como $\Delta_{B O}=\pi R_{i} R_{0} / A_{i}=$ $\pi /(4 \Delta)$. También definieron el parámetro adimensional $\epsilon$, que llamaron eficiencia de llenado, como $\epsilon=\pi\left(R_{0}^{2}-r_{0}^{2}\right) / \pi R_{0}^{2}=1-\left(r_{0} / R_{0}\right)^{2}$, cuya relación con las variables $\zeta$ de Giffen $\&$ Muraszew y $z$ de Taylor es $\epsilon=1-\zeta=1-z^{2}$. Sustituyendo en la ecuación de Bernoulli (2.62) se obtiene una expresión para el coeficiente de descarga $c_{d}$ en función de $\Delta_{B O}$ y $\epsilon$ :

$$
c_{d}=\left[\frac{1}{\epsilon^{2}}+\frac{\Delta_{B O}^{2}}{1-\epsilon}\right]^{-1 / 2}
$$

Aplicando también la condición de caudal máximo, $\partial c_{d} / \partial \epsilon=0$, luego:

$$
-\frac{1}{2}\left(\frac{\Delta_{B O}^{2}}{1-\epsilon}+\frac{1}{\epsilon^{2}}\right)^{-3 / 2}\left(\frac{\Delta_{B O}^{2}}{(1-\epsilon)^{2}}-\frac{2}{\epsilon^{3}}\right)=0
$$


de donde se obtiene:

$$
\Delta_{B O}=\frac{(1-\epsilon) \sqrt{2}}{\epsilon^{3 / 2}}
$$

Introduciendo (2.65) en (2.63):

$$
c_{d}=\frac{\epsilon^{3 / 2}}{(2-\epsilon)^{1 / 2}}
$$

El espesor de la lámina líquida en el orificio de salida, adimensionalizado con el radio de dicho orificio $R_{0}$, es:

$$
1-\frac{r_{0}}{R_{0}}=1-\sqrt{1-\epsilon}
$$

Los resultados obtenidos por Bayvel \& Orzechowski se muestran en la figura 2.6.

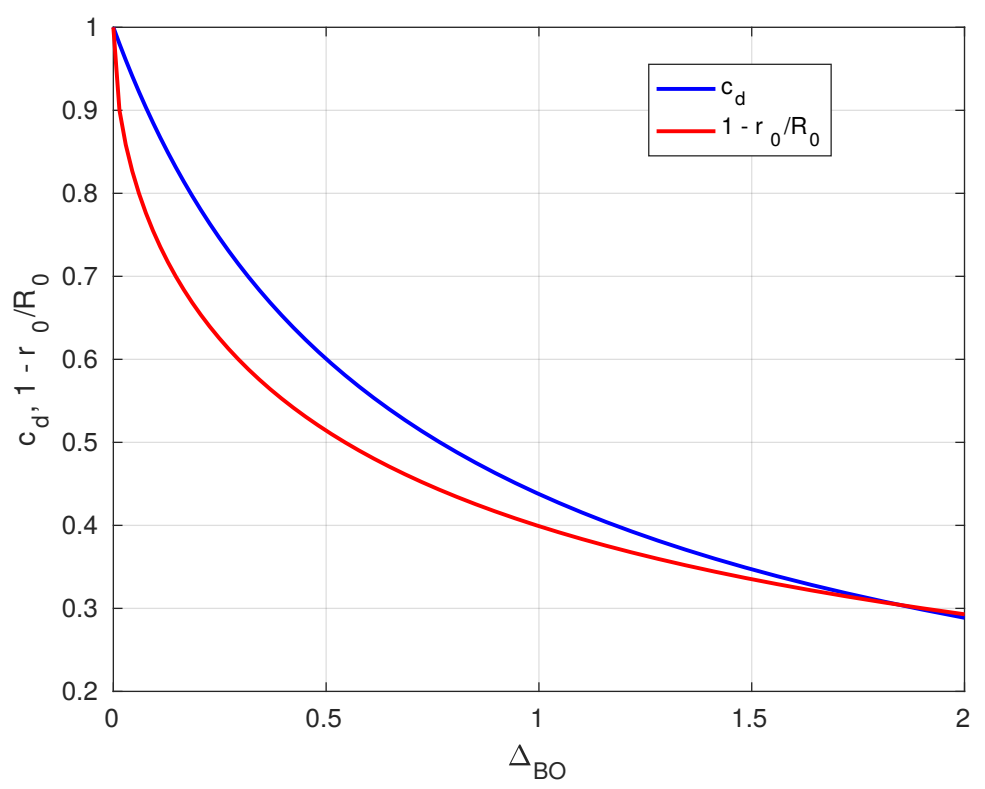

Figura 2.6 - Coeficiente de descarga $c_{d}$ (azul) y espesor adimensional de la lámina líquida en el orificio de salida $1-r_{0} / R_{0}$ (rojo) en función de la constante del atomizador $\Delta_{B O}$ (Bayvel \& Orzechowski 1993).

Todos estos autores emplean la condición de flujo crítico. Tanto Giffen \& Muraszew (1953) como Bayvel \& Orzechowski (1993) la aplican imponiendo que la velocidad y el espesor a la salida se han de adaptar de forma que el caudal sea máximo, como ya hizo Taylor (1948), por lo que es de esperar que los resultados obtenidos sean los mismos. Efectivamente, sustituyendo en las expresiones (2.55) y (2.66) para el coeficiente de descarga $c_{d}$ las equivalencias de las variables $\zeta$ y $\epsilon$ en función de la variable $z$, se obtiene la expresión (2.43) para el coeficiente de descarga $c_{d}$ calculada por Taylor (1948). Sin 
embargo, Nieuwkamp (1985) emplea la condición de flujo crítico por analogía con el flujo en un rebosadero, sustituyendo la aceleración de la gravedad por la aceleración centrífuga. Nieuwkamp considera un valor constante para la aceleración centrífuga, en concreto el que ésta tiene en la superficie del líquido en el orificio de salida. Pero sin embargo la aceleración centrífuga varía con la distancia radial, por lo que sus resultados sólo serán de aplicación en el caso de que el espesor de la lámina líquida en el orificio de salida sea pequeño. El espesor de la lámina líquida es menor cuando la constante del atomizador $\Delta$ es pequeña, por lo que los resultados obtenidos por Nieuwkamp se aproximarán más a los obtenidos por los otros autores según disminuya $\Delta$, como se observa en la figura 2.7.

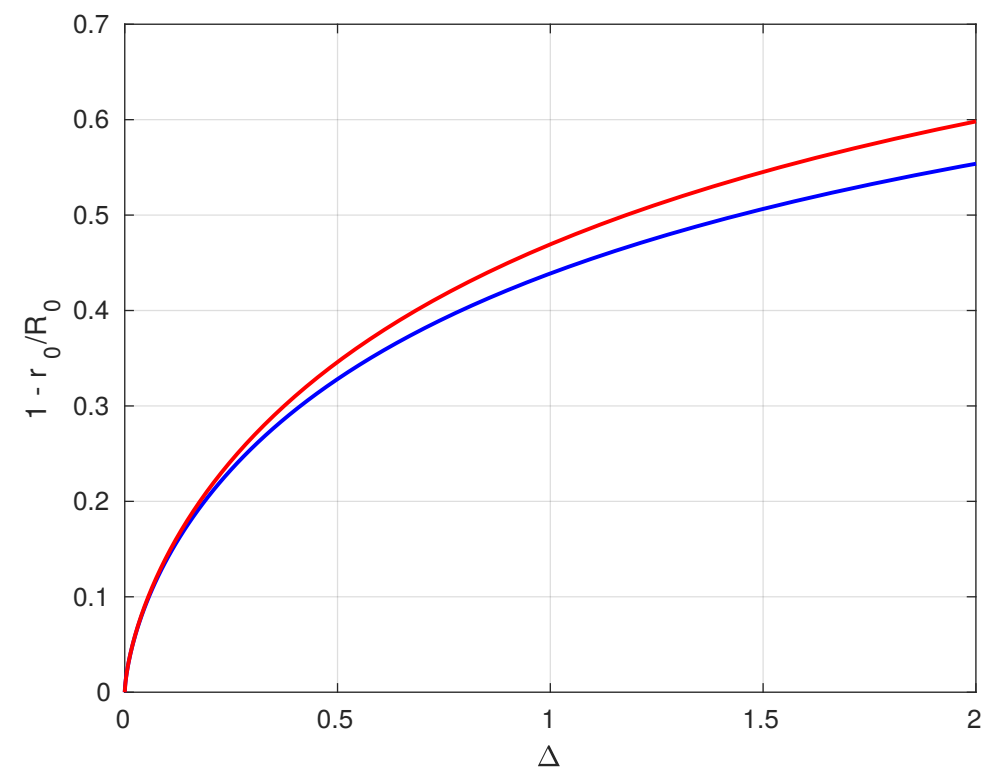

Figura 2.7 - Espesor adimensional de la lámina líquida en el orificio de salida $1-r_{0} / R_{0}$ en función de la constante del atomizador $\Delta$. Reultados obtenidos por Taylor (1948), Giffen \& Muraszew (1953) y Bayvel \& Orzechowski (1993) (azul) frente a los obtenidos por Nieuwkamp (1985) (azul).

Además, como ya se anticipó en la Sección 2.2, la velocidad axial en el orificio de salida, $u_{0}$, obtenida mediante la condición de caudal máximo, coincide con la velocidad de propagación de ondas largas dada por (2.120). En variables de Taylor, la velocidad axial es $u_{0}=x \Gamma /\left(2 \pi r_{s}\right)=x\left(R_{0} / r_{s}\right) \Gamma /\left(2 \pi R_{0}\right)=(x / y) \Gamma /\left(2 \pi R_{0}\right)$. Despejando $x$ de $(2.37)$ y sustituyendo el valor de $y$ obtenido en (2.42) se tiene:

$$
\frac{u_{0}}{\Gamma /\left(2 \pi R_{0}\right)}=\frac{1}{z^{2}} \sqrt{\frac{1}{2}\left(1-z^{2}\right)}
$$


que coincide con la velocidad de propagación de ondas que obtendremos en la Sección 2.5 para ondas en el orificio de salida en el límite $\left(k r_{0}, k R_{0}\right) \ll 1$, donde $k$ es el número de onda.

\subsubsection{Semiángulo de la lámina cónica}

Para calcular el semiángulo $\theta$ del cono que forma la lámina líquida, Taylor (1948) aplicó el balance de la cantidad de movimiento en dirección axial a un volúmen de control que comprende la lámina líquida entre el orificio de salida y una sección aguas abajo donde la contribución del término de presiones sea despreciable:

$$
\rho Q\left(u_{\infty}-u_{0}\right)=\int_{r_{0}}^{R_{0}} 2 \pi r p \mathrm{~d} r
$$

donde $u_{\infty}$ es la componente axial de la velocidad lejos del orificio de salida. La distribución de presión a lo largo del espesor de la lámina líquida en el orificio viene dada por la ecuación de Bernoulli:

$$
p=\frac{1}{2} \rho U_{\infty}^{2}\left(1-\frac{y^{2}}{r^{2} / R_{0}^{2}}-x^{2}\right)
$$

Introduciendo (2.69) en (2.68) resulta:

$$
\frac{u_{\infty}}{U_{\infty}}=x+\frac{y \sqrt{2} z^{2}}{\left(1-z^{2}\right)^{3 / 2}}\left[\frac{1}{2}\left(\frac{1}{z^{2}}-1\right)+\ln z\right]
$$

donde:

$$
\cos \theta=\frac{u_{\infty}}{U_{\infty}}
$$

El semiángulo de la lámina cónica calculado por Taylor se muestra en la figura 2.8. 


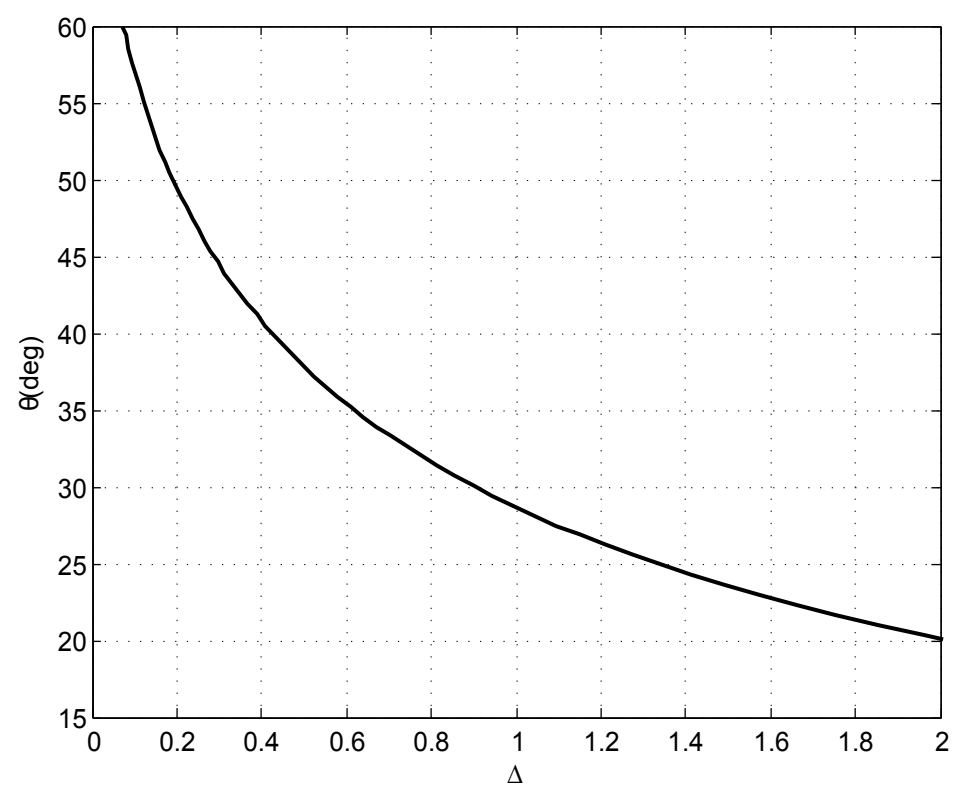

Figura 2.8 - Semiángulo de la lámina cónica $\theta$ en función de la constante del atomizador $\Delta$ (Taylor 1948).

Giffen \& Muraszew (1953) propusieron la siguiente expresión para el semiángulo de la lámina cónica:

$$
\sin \theta=\frac{\bar{w}_{0}}{U_{\infty}}
$$

donde $\bar{w}_{0}$ es la velocidad azimutal media, calculada dividiendo el transporte total de cantidad de movimiento de giro por el caudal:

$$
\bar{w}_{0}=\frac{\int_{r_{0}}^{R_{0}} 2 \pi r \rho u_{0} w \mathrm{~d} r}{\rho u_{0} \pi\left(R_{0}^{2}-r_{0}^{2}\right)}
$$

Teniendo en cuenta la definición (2.47) de Giffen \& Muraszew para la velocidad azimutal $w$, la expresión (2.73) se reduce a:

$$
\bar{w}_{0}=\frac{2 Q R_{s}}{A_{i}\left(R_{0}+r_{0}\right)}
$$

La velocidad azimutal media $\bar{w}_{0}$ obtenida en la expresión (2.74) coincide con la velocidad azimutal en el radio medio de la lámina líquida, $r=\left(R_{0}+r_{0}\right) / 2$. Expresando $U_{\infty}$ en función del coeficiente de descarga $c_{d}, U_{\infty}=Q /\left(\pi R_{0}^{2} c_{d}\right)$, y sustituyendo en (2.72), junto con (2.74), resulta:

$$
\sin \theta=\frac{2 \pi c_{d} R_{0}^{2} R_{s}}{A_{i}\left(R_{0}+r_{0}\right)}
$$


que en variables adimensionales es:

$$
\sin \theta=\frac{2 c_{d}}{\Delta_{G M}(1+\sqrt{\zeta})}
$$

Introduciendo el valor de la constante del atomizador $\Delta_{G M}$ y del coeficiente de descarga $c_{d}$ de las expresiones (2.53) y (2.55), respectivamente, en (2.76):

$$
\sin \theta=\frac{2 \sqrt{2} \zeta}{(1+\sqrt{\zeta}) \sqrt{1+\zeta}}
$$

El semiángulo de la lámina cónica calculado por Giffen \& Muraszew se muestra en la figura 2.9 .

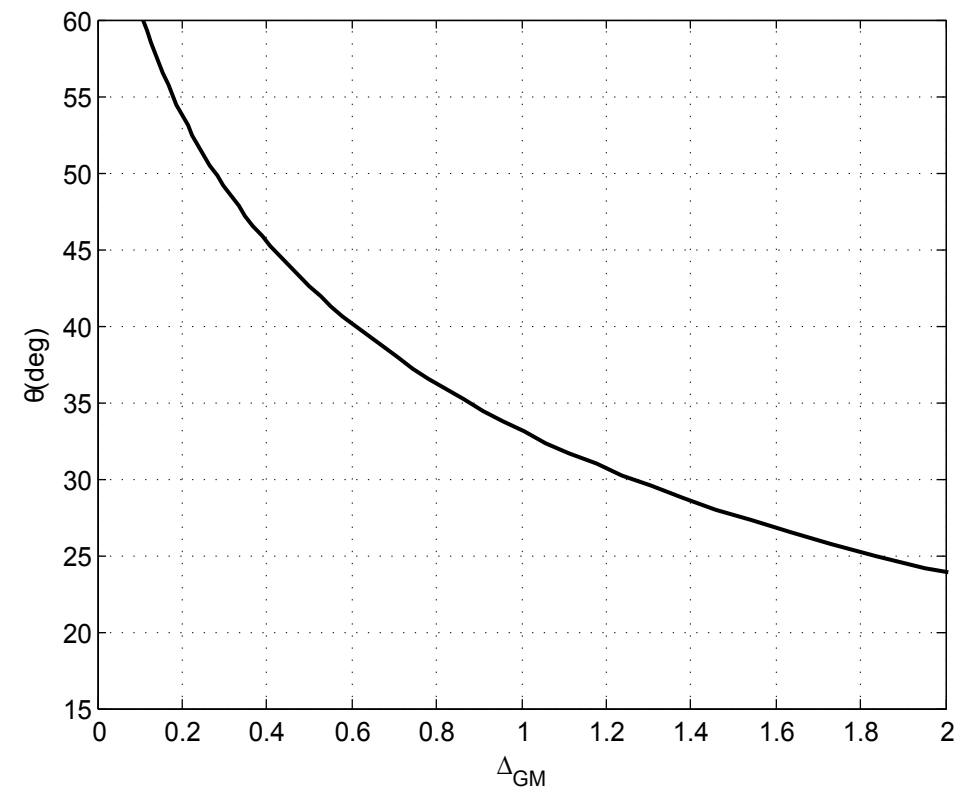

Figura 2.9 - Semiángulo de la lámina cónica $\theta$ en función de la constante del atomizador $\Delta_{G M}$ (Giffen \& Muraszew 1953).

Bayvel \& Orzechowski (1993) calcularon el semiángulo de la lámina cónica como la arcotangente de la relación entre las velocidades azimutal y axial en el orificio de salida:

$$
\tan \theta=\frac{\bar{w}_{0}}{u_{0}}
$$

donde, al igual que Giffen \& Muraszew, consideran que la velocidad azimutal media $\bar{w}_{0}$ se produce en el radio medio del espesor de la lámina, $r=\left(R_{0}+r_{0}\right) / 2$. Teniendo en cuenta las expresiones (2.4) y (2.49) para las velocidades azimutal y axial, respectivamente, se obtiene:

$$
\tan \theta=\frac{2 \pi\left(R_{0}-r_{0}\right) R_{i}}{A_{i}}
$$


Expresando (2.79) en función de la constante del atomizador $\Delta_{B O}$ y de la variable $\epsilon$ :

$$
\tan \theta=2 \Delta_{B O}(1-\sqrt{1-\epsilon})
$$

Sustituyendo (2.65) en (2.80):

$$
\tan \theta=\frac{2 \sqrt{2}(1-\epsilon)}{\sqrt{\epsilon}(1+\sqrt{1-\epsilon})}
$$

El semiángulo de la lámina cónica calculado por Bayvel \& Orzechowski se muestra en la figura 2.10 .

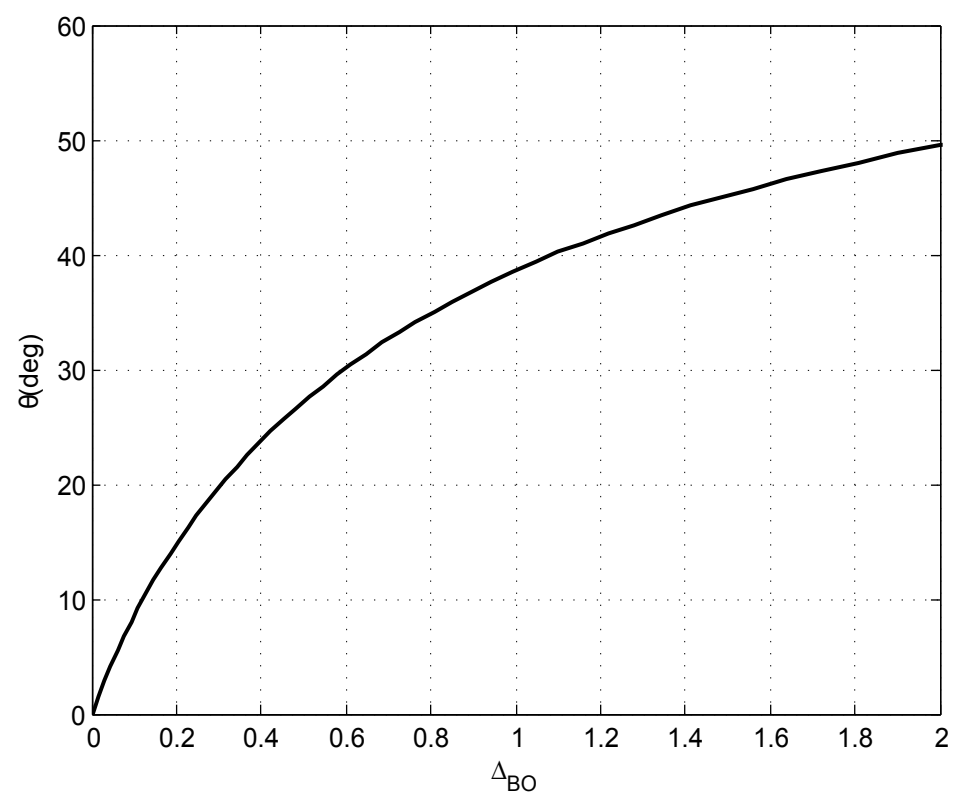

Figura 2.10 - Semiángulo de la lámina cónica $\theta$ en función de la constante del atomizador $\Delta_{B O}$ (Bayvel \& Orzechowski 1993).

Los resultados obtenidos por los autores anteriores para el semiángulo de la lámina cónica aparecen comparados en la figura 2.11 en función de la constante del atomizador $\Delta$. Puede observarse que los valores obtenidos para dicho semiángulo por Bayvel \& Orzechowski (1993) son superiores a los obtenidos por Taylor (1948) y por Giffen \& Muraszew (1953). Esto es debido a que Bayvel \& Orzechowski consideran que la velocidad axial en el infinito, $u_{\infty}$, es la misma que la velocidad axial en el orificio de salida, $u_{0}$. En los análisis de los otros autores, $u_{\infty}$ es mayor que $u_{0}$, por lo que obtienen valores más pequeños del semiángulo de la lámina cónica. Considerar que estas velocidades son iguales equivale a despreciar la distribución de presión a través de la lámina líquida en el orificio de salida, lo cual será más realista cuanto más delgada sea dicha lámina. Así pues, los resultados de 
Bayvel \& Orzechowski se aproximarán a los de los otros autores cuando el radio del núcleo de aire tienda al radio del orificio de salida, es decir, cuando la constante del atomizador tienda a cero, como se muestra en la figura 2.11.

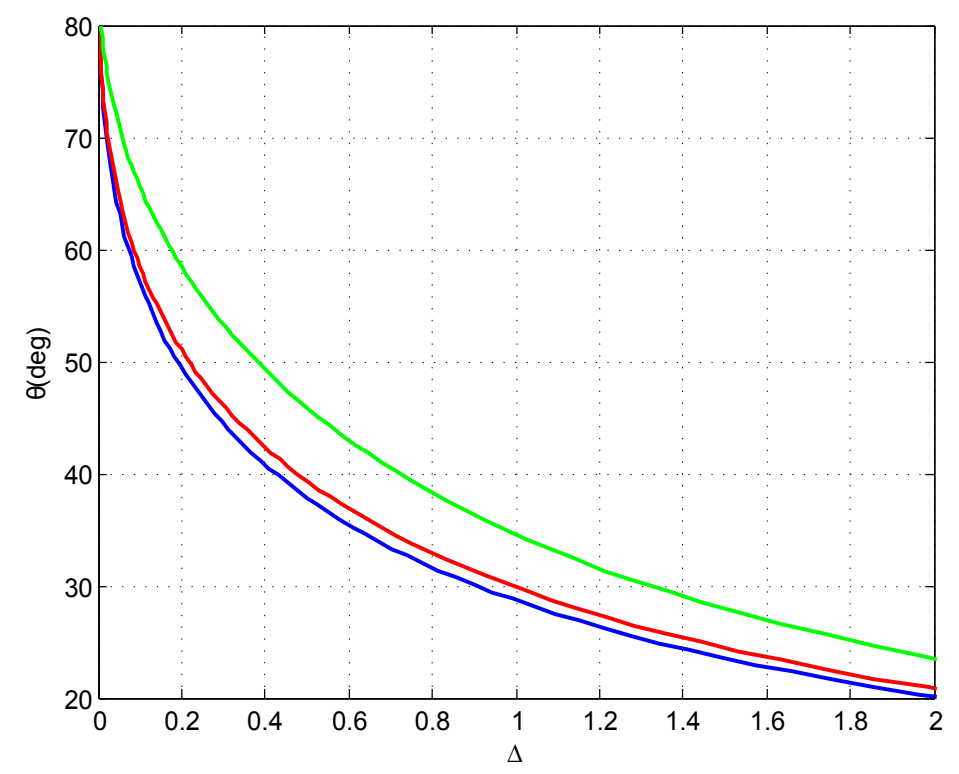

Figura 2.11 - Semiángulo de la lámina cónica $\theta$ en función de la constante del atomizador $\Delta$. Reultados obtenidos por Taylor (1948) (azul), Giffen \& Muraszew (1953) (rojo) y Bayvel \& Orzechowski (1993) (verde).

\subsection{Estudio numérico}

La aproximación cuasi-unidireccional predice que las características del flujo dependen únicamente de la constante del atomizador, y son independientes del resto de parámetros geométricos. Sin embargo, dichos parámetros pueden tener efecto en los resultados del problema irrotacional. Para analizar dicho efecto, se ha realizado un estudio numérico, para lo que se ha construido un modelo basado en el método de los elementos de contorno. En dicho estudio, se han escalado las variables espaciales con $R_{0}$ y las velocidades con $\Gamma /\left(2 \pi R_{0}\right)$.

La solución del problema (2.8) - (2.11), complementado con condiciones iniciales apropiadas, nunca es estacionaria porque la lámina se rompe a una cierta distancia del atomizador. En este capítulo analizaremos únicamente el flujo en la cámara del atomizador y el comienzo de la lámina. En el tratamiento numérico, para restringir el problema al interior 
de la cámara y a una región exterior donde el flujo es estacionario, se recurre a truncar artificialmente la lámina a una cierta distancia de la cámara, que se elige con la condición de que la lámina ya haya alcanzado la forma cónica mencionada antes. Se comprueba, en todo caso, que la solución estacionaria obtenida de este modo es independiente de la distancia a la que se trunca la lámina.

\subsubsection{El método de los elementos de contorno}

El método de los elementos de contorno (BEM, boundary-element method) es un método numérico válido para resolver la ecuación de Laplace (Pozrikidis 2002). La principal ventaja del método de los elementos de contorno frente a otros métodos alternativos es la reducción de la dimensión del problema, al transformar la ecuación de Laplace en una ecuación integral sobre la frontera del dominio donde se busca la solución. En contraste, otros métodos alternativos, como pueden ser los métodos de diferencias finitas o los métodos de elementos finitos, requieren discretizar todo el dominio, por lo que requieren mucho más cálculo para un mismo nivel de precisión.

La idea en que se basa el método de los elementos de contorno es expresar la solución como distribuciones de soluciones fundamentales de la ecuación de Laplace sobre la frontera del dominio. Estas soluciones fundamentales son funciones de Green que expresan el campo debido a una fuente o un dipolo localizados. Las densidades de estas distribuciones se calculan de forma que se satisfagan las condiciones de contorno especificadas.

\subsubsection{Formulación del método}

El problema a resolver está gobernado por la ecuación de Laplace para una función $\varphi(\mathbf{x}), \nabla^{2} \varphi=0$, definida en un volumen $V$ limitado por una superficie $\Sigma$, cuya normal exterior es $\mathbf{n}$. Sea $G\left(\mathbf{x}-\mathbf{x}^{\prime}\right)=1 /\left(4 \pi\left|\mathbf{x}-\mathbf{x}^{\prime}\right|\right)$ el potencial de una fuente puntual de intensidad unidad localizada en $\mathbf{x}^{\prime}$, que es la solución de $\nabla^{2} G=\delta\left(\mathbf{x}-\mathbf{x}^{\prime}\right)$. Las funciones $\varphi$ y $G$ satisfacen la primera identidad de Green:

$$
\nabla \cdot(G \nabla \varphi)=\nabla G \nabla \varphi+G \nabla^{2} \varphi
$$

Intercambiando los papeles de las funciones $\varphi$ y $G$ se obtiene:

$$
\nabla \cdot(\varphi \nabla G)=\nabla \varphi \nabla G+\varphi \nabla^{2} G
$$

Sustrayendo la expresión (2.83) a (2.82) se obtiene la segunda identidad de Green:

$$
G \nabla^{2} \varphi-\varphi \nabla^{2} G=\nabla \cdot(G \nabla \varphi-\varphi \nabla G)
$$


Si se tiene en cuenta que la función $\varphi$ satisface la ecuación de Laplace, y si se considera la definición de la función $G$, la expresión (2.84) da lugar a la siguiente relación:

$$
\nabla \cdot(\varphi \nabla G-G \nabla \varphi)=\varphi \delta\left(\mathbf{x}-\mathbf{x}^{\prime}\right)
$$

Integrando sobre el volumen $V$ y aplicando el teorema de Gauss:

$$
\int_{\Sigma} \varphi \mathbf{n} \cdot \nabla G \mathrm{~d} \sigma-\int_{\Sigma} G \mathbf{n} \cdot \nabla \varphi \mathrm{d} \sigma=\left\{\begin{array}{lll}
\varphi\left(\mathbf{x}^{\prime}\right) & \text { si } & \mathbf{x}^{\prime} \in V \\
0 & \text { si } & \mathbf{x}^{\prime} \notin V
\end{array}\right.
$$

Si $\mathbf{x}^{\prime}$ tiende a $\Sigma$ desde el interior de $V$, la primera integral de la expresión (2.85) puede descomponerse en la contribución de una semiesfera de centro $\mathbf{x}^{\prime}$ y radio $\epsilon$ muy pequeño, y la contribución del resto de $\Sigma$ (ver figura 2.12). La contribución de la semiesfera es la siguiente:

$$
\int_{\text {Semiesfera }} \varphi \mathbf{n} \cdot \nabla G \mathrm{~d} \sigma \approx \varphi\left(\mathbf{x}^{\prime}\right) \int_{\text {Semiesfera }} \frac{1}{4 \pi \epsilon^{2}} \mathrm{~d} \sigma=\frac{1}{2} \varphi\left(\mathbf{x}^{\prime}\right)
$$

Así pues:

$$
\int_{\Sigma} \varphi \mathbf{n} \cdot \nabla G \mathrm{~d} \sigma=\frac{1}{2} \varphi\left(\mathbf{x}^{\prime}\right)+\int_{P V} \varphi \mathbf{n} \cdot \nabla G \mathrm{~d} \sigma
$$

En la expresión (2.86) $P V$ designa el valor principal de la integral, que es la contribución de $\Sigma$ excluyendo un disco de centro $\mathrm{x}^{\prime}$ y radio $\epsilon \rightarrow 0$. Si se lleva esta expresión a la expresión (2.85) se obtiene la siguiente ecuación integral:

$$
\varphi\left(\mathbf{x}^{\prime}\right)=2 \int_{P V_{\Sigma}} \varphi \mathbf{n} \cdot \nabla G \mathrm{~d} \sigma-2 \int_{\Sigma} G \mathbf{n} \cdot \nabla \varphi \mathrm{d} \sigma \quad \text { con } \quad \mathbf{x}^{\prime} \in \Sigma
$$

Sin embargo, si $\mathbf{x}^{\prime}$ tiende a una arista de $\Sigma$ en la que dicha superficie forma un ángulo $\alpha$ visto desde el interior de $V$, entonces la superficie que rodea a $\mathbf{x}^{\prime}$ no es una semiesfera sino un sector esférico (ver figura 2.12), y la ecuación integral se vería modificada de la siguiente manera:

$$
\begin{gathered}
\int_{\text {Sector }} \varphi \mathbf{n} \cdot \nabla G \mathrm{~d} \sigma \approx \varphi\left(\mathbf{x}^{\prime}\right) \int_{\text {Sector }} \frac{1}{4 \pi \epsilon^{2}} \mathrm{~d} \sigma=\left(1-\frac{\alpha}{2 \pi}\right) \varphi\left(\mathbf{x}^{\prime}\right) \\
\varphi\left(\mathbf{x}^{\prime}\right)=\frac{2 \pi}{\alpha} \int_{P V_{\Sigma}} \varphi \mathbf{n} \cdot \nabla G \mathrm{~d} \sigma-\frac{2 \pi}{\alpha} \int_{\Sigma} G \mathbf{n} \cdot \nabla \varphi \mathrm{d} \sigma \quad \text { con } \quad \mathbf{x}^{\prime} \in \Sigma
\end{gathered}
$$



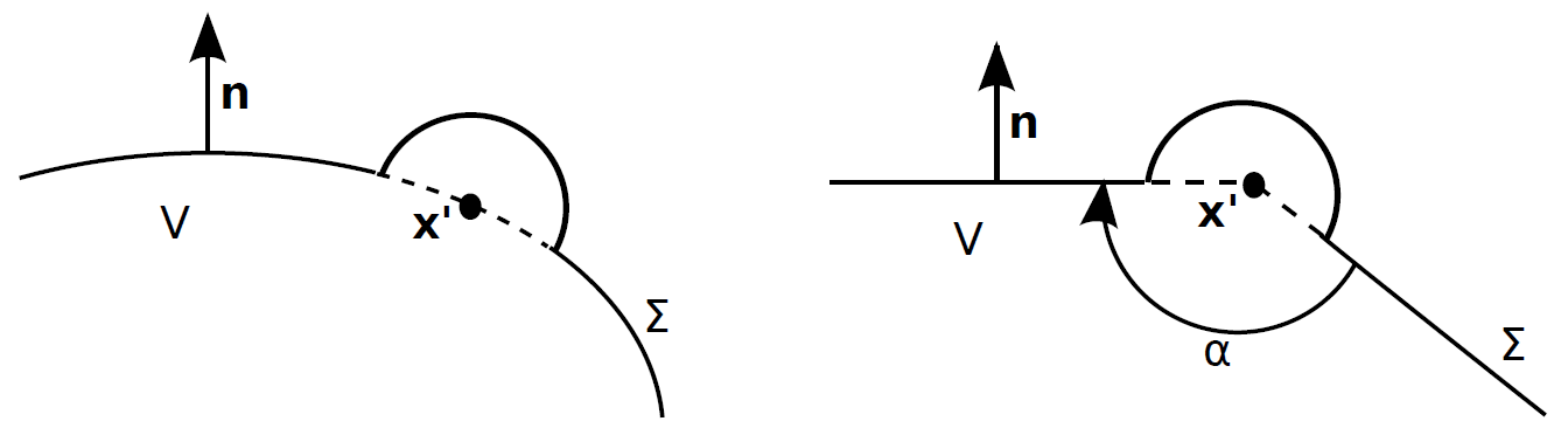

Figura 2.12 - Descomposición de la superficie $\Sigma$ en una parte principal y una semiesfera de radio $\epsilon$ alrededor del punto $\mathbf{x}^{\prime}$. A la derecha se muestra que si el punto $\mathbf{x}^{\prime}$ se encuentra en una arista de la superficie $\Sigma$ entonces la superficie esférica que lo rodea no es una semiesfera.

Si la función $\varphi$ y la superficie $\Sigma$ tienen simetría de revolución alrededor de un eje $x$, y se usan coordenadas cilíndricas $(x, r, \phi)$, las ecuaciones de superficie en $(2.87)$ pueden reducirse a integrales de línea sobre una generatriz de $\Sigma$ :

$$
\varphi\left(x^{\prime}, r^{\prime}\right)=\oint_{C} \frac{\partial \varphi}{\partial n}(x, r) \hat{G}\left(x, r, x^{\prime}, r^{\prime}\right) r \mathrm{~d} s-\oint_{P V_{C}} \varphi(x, r) \frac{\partial \hat{G}}{\partial n}\left(x, r, x^{\prime}, r^{\prime}\right) r \mathrm{~d} s
$$

En la anterior expresión, la función $\hat{G}$ y su derivada respecto a la dirección normal se definen de la siguiente manera:

$$
\begin{gathered}
\hat{G}=\frac{2}{\pi} \frac{K(m)}{\sqrt{\left(x-x^{\prime}\right)^{2}+\left(r+r^{\prime}\right)^{2}}} \\
\frac{\partial \hat{G}}{\partial n}=\frac{1}{\sqrt{\left(x-x^{\prime}\right)^{2}+\left(r+r^{\prime}\right)^{2}}} \frac{1}{\pi r}\left(\frac{r^{\prime 2}-r^{2}+\left(x-x^{\prime}\right)^{2}}{\left(x-x^{\prime}\right)^{2}+\left(r-r^{\prime}\right)^{2}} E(m)-K(m)\right) n_{r} \\
+\frac{2}{\pi} \frac{1}{\sqrt{\left(x-x^{\prime}\right)^{2}+\left(r+r^{\prime}\right)^{2}}} \frac{\left(x^{\prime}-x\right) E(m) n_{x}}{\left(x-x^{\prime}\right)^{2}+\left(r-r^{\prime}\right)^{2}} \\
m=\frac{4 r r^{\prime}}{\left(x-x^{\prime}\right)^{2}+\left(r+r^{\prime}\right)^{2}}
\end{gathered}
$$

Las funciones $\mathrm{K}(\mathrm{m})$ y $\mathrm{E}(\mathrm{m})$ son las integrales elípticas completas de primera y segunda especie, respectivamente:

$$
\begin{gathered}
K(m)=\int_{0}^{\pi / 2} \frac{\mathrm{d} \phi}{\sqrt{1-m \sin ^{2} \phi}} \\
E(m)=\int_{0}^{\pi / 2} \sqrt{1-m \sin ^{2} \phi} \mathrm{d} \phi
\end{gathered}
$$




\subsubsection{Simulación}

La frontera del líquido tiene varias partes, donde los datos e incógnitas son distintos. En las paredes internas del atomizador y en la sección de entrada el potencial de velocidades $\varphi$ es desconocido, mientras que son conocidos los valores de su derivada respecto a la normal, $\partial \varphi / \partial n$. En la superficie libre lo que se conoce es $\varphi$, siendo incógnita $\partial \varphi / \partial n$. Para resolver el problema, partiendo de una posición inicial de la superficie libre, se resuelve la ecuación de Laplace para el potencial de velocidad, con lo que se obtendrá la distribución de velocidades en la superficie libre. Con esta distribución de velocidades se recalculará la posición de la superficie libre, como se explica más adelante, y se repite el proceso hasta obtener la posición estacionaria de la misma.

Para resolver la ecuación de Laplace para el potencial de velocidad, se ha discretizado toda la frontera del líquido en un número $n$ de nodos. A cada uno de estos nodos, $j=1, \ldots, n$, le corresponderán unas coordenadas espaciales, $x\left(s_{j}\right)$ y $r\left(s_{j}\right)$, un valor del potencial de velocidades, $\varphi\left(s_{j}\right)$, y un valor de la derivada del mismo respecto a la normal, $\partial \varphi\left(s_{j}\right) / \partial n$, donde $s_{j}$ es el valor que toma el parámetro longitud de arco $s$ en el nodo $j$. Los valores de las coordenadas espaciales para puntos situados entre dos nodos consecutivos se obtienen mediante interpolación por splines cúbicos.

Para calcular las integrales se ha usado integración gaussiana en cada intervalo. Para reducir las integrales al intervalo $[-1,1]$, expresamos $s$ en función de una nueva variable $\xi$ tal que $s=\left(s_{j}-s_{j-1}\right) \xi / 2+\left(s_{j}+s_{j-1}\right) / 2$ :

$$
\int_{s_{j-1}}^{s_{j}} f(s) \mathrm{d} s=\frac{s_{j}-s_{j-1}}{2} \int_{-1}^{1} f(s(\xi)) \mathrm{d} \xi=\frac{s_{j}-s_{j-1}}{2} \sum_{k=1}^{N} W_{k} f\left(s\left(\xi_{k}\right)\right) \quad \text { con } \quad \sum_{k=1}^{N} W_{k}=2
$$

siendo $W_{k}$ y $\xi_{k}$ pesos y puntos de colocación conocidos, respectivamente. Las integrales sobre los intervalos adyacentes al punto $\left(x^{\prime}, r^{\prime}\right)$ requieren un tratamiento especial para tener en cuenta las singularidades de la función $\hat{G}$ y su derivada cuando $(x, r) \rightarrow\left(x^{\prime}, r^{\prime}\right)$. Definiendo $\delta x=x-x^{\prime} \rightarrow 0$ y $\delta r=r-r^{\prime} \rightarrow 0$, entonces se tiene que:

$$
\begin{gathered}
m=1-\frac{1}{4} \frac{(\delta x)^{2}+(\delta r)^{2}}{r^{2}}+\ldots \\
K(m)=-\frac{1}{2} \ln \left[(\delta x)^{2}+(\delta r)^{2}\right]+O(1)=-\ln (\delta s)+O(1)
\end{gathered}
$$

Así pues, las contribuciones de las partes singulares de $\hat{G}$ y su derivada normal $\partial \hat{G} / \partial n$ a las integrales sobre los intervalos $\left(s_{i-1}, s_{i}\right)$ y $\left(s_{i}, s_{i+1}\right)$ en la ecuación integral aplicada al nodo $\left[x\left(s_{i}\right), r\left(s_{i}\right)\right]$ son:

$$
\int_{s_{i-1}}^{s_{i}} \frac{\partial \varphi}{\partial n} \frac{2}{\pi} \frac{-\ln \left(s_{i}-s\right)}{2 r} r \mathrm{~d} s=-\frac{1}{\pi} \frac{\partial \varphi\left(s_{i}\right)}{\partial n}\left(s_{i}-s_{i-1}\right)\left[\ln \left(s_{i}-s_{i-1}\right)-1\right]
$$




$$
\begin{aligned}
& \int_{s_{i}}^{s_{i+1}} \frac{\partial \varphi}{\partial n} \frac{2}{\pi} \frac{-\ln \left(s-s_{i}\right)}{2 r} r \mathrm{~d} s=-\frac{1}{\pi} \frac{\partial \varphi\left(s_{i}\right)}{\partial n}\left(s_{i+1}-s_{i}\right)\left[\ln \left(s_{i+1}-s_{i}\right)-1\right] \\
& \int_{s_{i-1}}^{s_{i}} \varphi \frac{2}{\pi} \frac{-\ln \left(s_{i}-s\right)}{(2 r)^{2}} n_{r} r \mathrm{~d} s=-\frac{n_{r}}{2 \pi r} \varphi\left(s_{i}\right)\left(s_{i}-s_{i-1}\right)\left[\ln \left(s_{i}-s_{i-1}\right)-1\right] \\
& \int_{s_{i}}^{s_{i+1}} \varphi \frac{2-\ln \left(s-s_{i}\right)}{\pi} n_{r} r \mathrm{~d} s=-\frac{n_{r}}{2 \pi r} \varphi\left(s_{i}\right)\left(s_{i+1}-s_{i}\right)\left[\ln \left(s_{i+1}-s_{i}\right)-1\right]
\end{aligned}
$$

Si las integrales sobre estos intervalos se evalúan con el mismo método numérico que las integrales sobre los demás intervalos, no se obtienen los resultados correctos mostrados antes, sino:

$$
\begin{aligned}
& \int_{s_{i-1}}^{s_{i}} \frac{\partial \varphi}{\partial n} \frac{2}{\pi} \frac{-\ln \left(s_{i}-s\right)}{2 r} r \mathrm{~d} s=-\frac{1}{\pi} \frac{\partial \varphi\left(s_{i}\right)}{\partial n}\left(s_{i}-s_{i-1}\right)\left[\ln \left(s_{i}-s_{i-1}\right)+\frac{1}{2} \sum_{k=1}^{N} W_{k} \ln \frac{1+\xi_{k}}{2}\right] \\
& \int_{s_{i}}^{s_{i+1}} \frac{\partial \varphi}{\partial n} \frac{2}{\pi} \frac{-\ln \left(s-s_{i}\right)}{2 r} r \mathrm{~d} s=-\frac{1}{\pi} \frac{\partial \varphi\left(s_{i}\right)}{\partial n}\left(s_{i+1}-s_{i}\right)\left[\ln \left(s_{i+1}-s_{i}\right)+\frac{1}{2} \sum_{k=1}^{N} W_{k} \ln \frac{1+\xi_{k}}{2}\right] \\
& \int_{s_{i-1}}^{s_{i}} \varphi \frac{2}{\pi} \frac{-\ln \left(s_{i}-s\right)}{(2 r)^{2}} n_{r} r \mathrm{~d} s=-\frac{n_{r}}{2 \pi r} \varphi\left(s_{i}\right)\left(s_{i}-s_{i-1}\right)\left[\ln \left(s_{i}-s_{i-1}\right)+\frac{1}{2} \sum_{k=1}^{N} W_{k} \ln \frac{1+\xi_{k}}{2}\right] \\
& \int_{s_{i}}^{s_{i+1}} \varphi \frac{2}{\pi} \frac{-\ln \left(s-s_{i}\right)}{(2 r)^{2}} n_{r} r \mathrm{~d} s=-\frac{n_{r}}{2 \pi r} \varphi\left(s_{i}\right)\left(s_{i+1}-s_{i}\right)\left[\ln \left(s_{i+1}-s_{i}\right)+\frac{1}{2} \sum_{k=1}^{N} W_{k} \ln \frac{1+\xi_{k}}{2}\right]
\end{aligned}
$$

Así pues, en estos intervalos las integrales calculadas numéricamente deben corregirse añadiendo el término adecuado.

Aplicando la ecuación integral (2.89) a cada uno de los $n$ nodos se obtiene un sistema de $n$ ecuaciones lineales en función de $\operatorname{los} n$ valores del potencial $\varphi$ y de su derivada respecto a la normal normal $\partial \varphi / \partial n$ en cada nodo. En las paredes internas del atomizador y en la sección de entrada son conocidos los valores de $\partial \varphi / \partial n$, mientras que en la superficie libre se conoce el potencial $\varphi$. Así pues, se tiene un sistema de $n$ ecuaciones con $n$ incógnitas: los valores de $\varphi$ en los nodos de las paredes internas del atomizador y de la sección de entrada, y los valores de $\partial \varphi / \partial n$ en los nodos de la superficie libre.

El método de resolución que se ha seguido es el siguiente:

1. Se resuelve el sistema, obteniéndose los valores de $\varphi$ en los nodos de las paredes internas del atomizador y de la sección de entrada, y los valores de $\partial \varphi / \partial n$ en los nodos de la superficie libre.

2. Con los valores obtenidos de $\partial \varphi / \partial n$ en los nodos de la superficie libre, se calculan las componentes axial y radial de la velocidad en dichos nodos. Expresándolas en 
función de las componentes tangencial, $v_{t}$, y normal, $v_{n}$, a la superficie libre del líquido, se obtiene:

$$
\begin{aligned}
& u=v_{t} \cos \theta+v_{n} \sin \theta \\
& v=v_{t} \sin \theta-v_{n} \cos \theta
\end{aligned}
$$

donde $\theta$ es el ángulo que forma la tangente a la superficie libre con la dirección axial:

$$
\begin{aligned}
\cos \theta & =\frac{\partial x / \partial s}{\sqrt{(\partial x / \partial s)^{2}+(\partial r / \partial s)^{2}}} \\
\sin \theta & =\frac{\partial r / \partial s}{\sqrt{(\partial x / \partial s)^{2}+(\partial r / \partial s)^{2}}}
\end{aligned}
$$

Expresando las componentes tangencial y normal en función de las derivadas del potencial $\varphi$ :

$$
\begin{gathered}
v_{t}=\frac{1}{\sqrt{(\partial x / \partial s)^{2}+(\partial r / \partial s)^{2}}} \frac{\partial \varphi}{\partial s} \\
v_{n}=\frac{\partial \varphi}{\partial n}
\end{gathered}
$$

Así pues, se obtiene finalmente:

$$
\begin{aligned}
& u=\frac{1}{\sqrt{(\partial x / \partial s)^{2}+(\partial r / \partial s)^{2}}}\left(\frac{\partial x / \partial s}{\sqrt{(\partial x / \partial s)^{2}+(\partial r / \partial s)^{2}}} \frac{\partial \varphi}{\partial s}+(\partial r / \partial s) \frac{\partial \varphi}{\partial n}\right) \\
& v=\frac{1}{\sqrt{(\partial x / \partial s)^{2}+(\partial r / \partial s)^{2}}}\left(\frac{\partial r / \partial s}{\sqrt{(\partial x / \partial s)^{2}+(\partial r / \partial s)^{2}}} \frac{\partial \varphi}{\partial s}-(\partial x / \partial s) \frac{\partial \varphi}{\partial n}\right)
\end{aligned}
$$

3. Con los valores obtenidos de $\partial \varphi / \partial n$ en los nodos de la superficie libre, se calcula la variación con respecto al tiempo del potencial de velocidades, $\partial \varphi / \partial t$, en dichos nodos, aplicando la ecuación de Bernoulli no estacionaria.

$$
\frac{\mathrm{d} \varphi}{\mathrm{d} t}=\frac{1}{2}\left[\left(\frac{1}{\sqrt{(\partial x / \partial s)^{2}+(\partial r / \partial s)^{2}}} \frac{\partial \varphi}{\partial s}\right)^{2}+\left(\frac{\partial \varphi}{\partial n}\right)^{2}-\frac{1}{r^{2}}+\frac{1}{r_{s}^{2}}+\left(\frac{\partial \varphi}{\partial n}\right)_{s}^{2}\right]
$$

4. Una vez calculados los valores de las componentes axial y radial de la velocidad, $u, v$, y de la variación del potencial de velocidades con respecto al tiempo, $\partial \varphi / \partial t$, en los nodos de la superficie libre, se obtienen las posiciones de dichos nodos y su potencial en el siguiente paso de tiempo.

5. Con la nueva posición de la superficie libre definida, se redistribuyen los nodos a lo largo de la misma.

6. Se repiten todos los anteriores pasos hasta que la superficie libre alcance su posición estacionaria. 


\subsubsection{Resultados del estudio numérico}

Se ha estudiado, mediante el modelo numérico antes descrito, el efecto de los diferentes parámetros geométricos del atomizador. En concreto, se ha analizado el efecto de la longitud de la cámara $\left(L_{s}\right)$, del radio de la cámara $\left(R_{s}\right)$ y de la longitud del orificio de salida $\left(L_{0}\right)$, todos ellos escalados con el radio del orificio de salida $\left(R_{0}\right)$, así como el efecto del ángulo de la contracción $(\alpha)$. Para ello, se ha modificado el valor de cada uno de estos parámetros manteniendo constantes los valores de los demás y de la constante del atomizador, para poder observar el efecto de todos ellos independientemente.

\section{Validación del modelo numérico}

Como ya se mencionó, la solución del problema nunca es estacionaria, pues la lámina líquida se extiende continuamente con el tiempo aguas abajo de la cámara, por lo que se ha recurrido a truncar artificialmente dicha lámina para restringir el problema al interior de la cámara y a una región exterior donde el flujo es estacionario. La distancia de la cámara a la que se trunca la lámina se ha elegido con la condición de que dicha lámina ya haya alcanzado su forma cónica. Primeramente, se ha comprobado que la solución estacionaria obtenida de este modo es independiente de la distancia a la que se ha truncado la lámina. En la figura 2.13 se muestra la superficie libre del líquido para varios valores de dicha distancia. Puede observarse que los resultados obtenidos son los mismos para las distintas distancias de truncamiento simuladas.

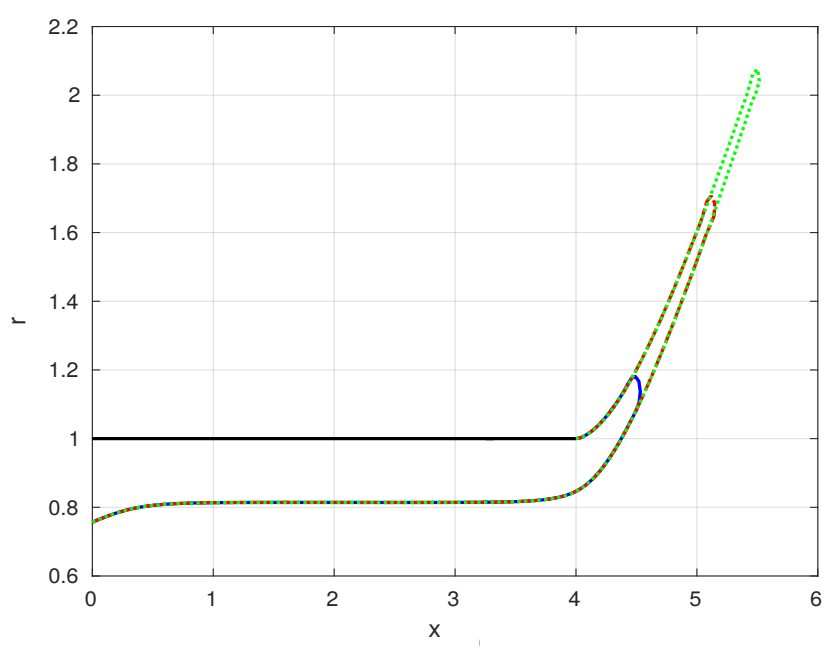

Figura 2.13 - Superficie libre del líquido para varios valores de la distancia de corte de la lámina cónica, con: $\Delta=0,196, L_{s}=2, R_{s}=2, L_{0}=4, \alpha=75$. 
La teoría cuasi-unidireccional predice que los resultados dependen únicamente de la constante del atomizador. Para poder ver esta dependencia con nuestro modelo numérico, y para comprobar su validez, se ha simulado una configuración del atomizador con sus parámetros geométricos fijos, y se ha variado el valor del caudal adimensional, que se traduce en una variación de la constante del atomizador. Los resultados obtenidos para las características del flujo se muestran en la figura 2.14, en la que puede observarse que se ajustan bastante bien a los valores que predice la teoría cuasi-unidireccional de Taylor (1948).
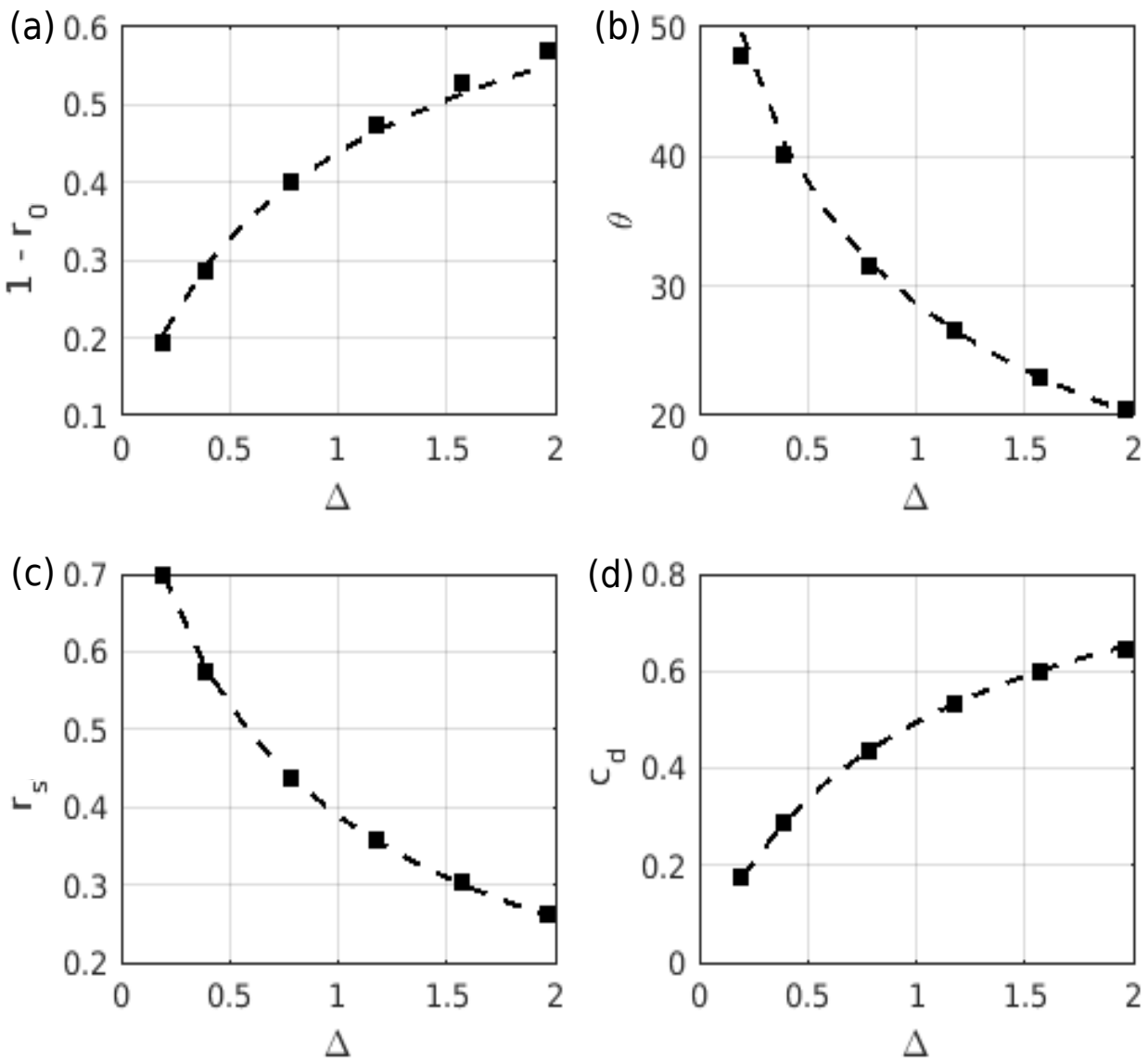

Figura 2.14 - (a) Espesor adimensional de la lámina líquida en el orificio de salida, $1-r_{0}$, (b) semiángulo de la lámina cónica, $\theta$, (c) radio adimensional del núcleo de aire en la cámara, $r_{s}$, y (d) coeficiente de descarga, $c_{d}$, en función de la constante del atomizador. Resultados numéricos (cuadrados) frente a resultados de la teoría cuasiunidireccional (Taylor 1948) (líneas discontínuas). El resto de los parámetros: $L_{s}=2$, $R_{s}=2, L_{0}=1, \alpha=75$. 


\section{Condición de flujo crítico a la salida}

El flujo en el orificio de salida se aproxima más al flujo unidireccional cuanto más esbelta sea la lámina líquida en dicho orificio, esto es, cuando la relación entre su espesor y la longitud del orificio de salida sea menor. Como se observa en la figura 2.14(a), este espesor disminuye cuando lo hace la constante del atomizador. En la figura 2.15 se representa la velocidad axial $u_{0}$ a lo largo del orificio de salida, tanto en la superficie del líquido como en la pared de dicho orificio, además de una velocidad axial media definida como el cociente entre el caudal y el área de cada sección transversal. Puede observarse que la velocidad axial en el orifico de salida es más uniforme cuanto menor es la constante del atomizador, esto es, cuanto más delgada es la lámina líquida.
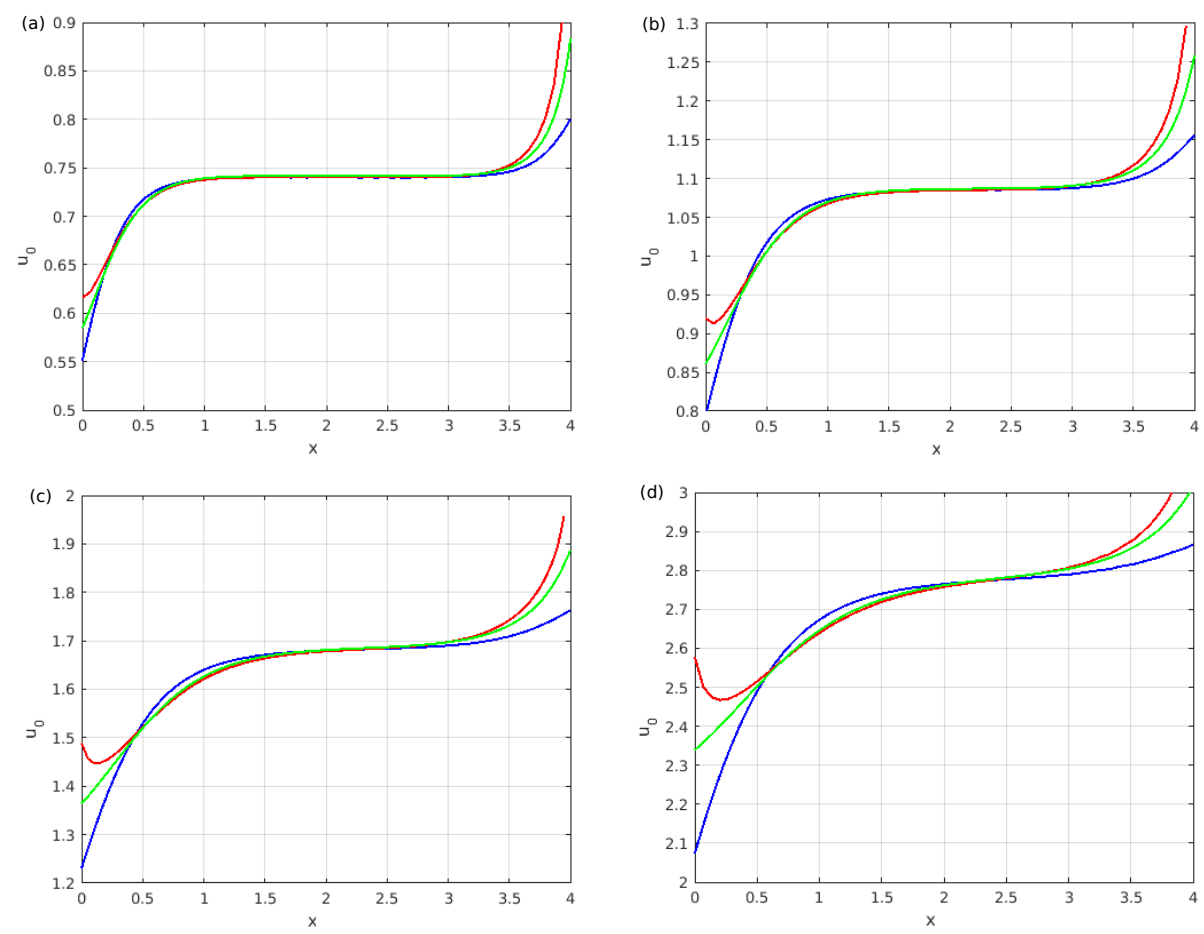

Figura 2.15 - Velocidad axial $u_{0}$ a lo largo del orificio de salida, para varios valores de la constante del atomizador: (a) $\Delta=0,1963$, (b) $\Delta=0,3927$, (c) $\Delta=0,7854$, (d) $\Delta=1,5708$. En azul, velocidad axial en la superficie del líquido; en rojo, velocidad axial en la pared del orificio; en verde, velocidad axial media. El resto de los parámetros: $L_{s}=2, R_{s}=2, L_{0}=4, \alpha=75$.

Para que el flujo sea crítico, la velocidad axial del mismo en dicho orificio, $u_{0}$, ha de coincidir con la velocidad de propagación de ondas $c_{0}$ obtenida en la Sección 2.5 y dada por 
la expresión (2.120). En la figura 2.16 se muestra la diferencia entre $u_{0} \mathrm{y} c_{0}$ en función del radio del núcleo de aire en el orificio de salida. Se observa que esta diferencia disminuye cuando aumenta el radio del núcleo de aire en el orificio de salida, es decir, cuando la lámina líquida en dicho orificio es más esbelta y el flujo se aproxima más al unidireccional. Sin embargo, debido a limitaciones de capacidad de cálculo, en las simulaciones no se ha conseguido que la velocidad en el orificio de salida alcance la velocidad de propagación de las ondas, pues para ello se habrían de simular casos con la película líquida a la salida más esbelta, lo que implica aumentar el número de nodos del modelo considerablemente.

Los valores relativamente grandes de $\left(c_{0}-u_{0}\right) / u_{0}$ en la figura 2.16 para los mayores valores de $r_{0}$ para los que se ha calculado la solución se deben probablemente a que tanto $u_{0}$ como $c_{0}$ tienden a cero cuando $r_{0} \rightarrow 1$. La solución en este límite asintótico puede calcularse fácilmente teniendo en cuenta que pequeños valores de $1-r_{0}$ están asociados a pequeños valores de $1-r_{s}$, siendo $r_{s}$ el radio adimensional del núcleo de aire en la base de la cámara, donde $\tilde{\mathbf{v}}=0$, y por tanto a pequeños valores de $1-r_{a c}$ en cualquier punto de la superficie del líquido. Usando la aproximación $1 /\left(2 r_{a c}^{2}\right) \approx 1 / 2-\left(r_{a c}-1\right)$ en el término de fuerza másica aparente de la ecuación de Bernoulli sobre la superficie del líquido, esta ecuación se reduce a

$$
\frac{1}{2}\left|\nabla \varphi_{a c}\right|^{2}+1-r_{a c}=1-r_{s}
$$

en las variables adimensionales de esta sección. En esta aproximación, la fuerza másica aparente debida a la velocidad azimutal del líquido es uniforme, siendo estrictamente análoga a la gravedad en el flujo sobre un rebosadero (con $g=1$ en variables adimensionales). La velocidad del líquido a la salida de la cámara, donde $r_{a c}=r_{0}$, es $u_{0}=\sqrt{2\left(r_{0}-r_{s}\right)}$, mientras que la velocidad de las ondas en esta superficie es $c_{0}=\sqrt{1-r_{0}}$. La condición de que el flujo sea crítico $\left(u_{0}=c_{0}\right)$ determina

$$
1-r_{0}=\frac{2}{3}\left(1-r_{s}\right)
$$

de modo que

$$
u_{0}=c_{0}=\sqrt{\frac{2}{3}\left(1-r_{s}\right)}
$$

El caudal adimensionalizado con $\Gamma R_{0} / 2 \pi$ es $2 \pi u_{0}\left(1-r_{0}\right)=2 \pi(2 / 3)^{3 / 2}\left(1-r_{s}\right)^{3 / 2}$, que corresponde a un valor $\Delta=(\pi / 2)(2 / 3)^{3 / 2}\left(1-r_{s}\right)^{3 / 2}$ de la constante del atomizador. Así pues:

$$
1-r_{s}=\frac{3}{2}\left(\frac{2 \Delta}{\pi}\right)^{2 / 3} ; 1-r_{0}=\left(\frac{2 \Delta}{\pi}\right)^{2 / 3} ; u_{0}=\left(\frac{2 \Delta}{\pi}\right)^{1 / 3} ; \quad c_{0}=\frac{4 \Delta}{\pi} \quad \text { cuando } \Delta \ll 1
$$


En el límite opuesto, $r_{s} \ll 1$, la velocidad azimutal es del orden de su máximo $\left(1 / r_{s}\right)$ a distancias de orden $r_{s}$ del eje de la cámara, pero puede despreciarse a distancias mayores. La sobrepresión en la cámara adimensionalizada con $\rho\left(\Gamma /\left(2 \pi R_{0}\right)\right)^{2}$ es $1 /\left(2 r_{s}^{2}\right)$, la descarga ocurre aproximadamente como si el líquido no girase y no hubiese núcleo de aire, lo que da lugar a un coeficiente de descarga $c_{d 0}$ dependiente únicamente de la forma de la contracción y aproximadamente igual a la unidad si $L_{0}$ es moderadamente grande comparado con $R_{0}$ en la figura 2.1. En función de la constante del atomizador, $r_{s}=\pi c_{d 0} /(4 \Delta)$. Obsérvese que estos resultados se obtienen sin emplear ninguna condición de criticalidad a la salida de la cámara.

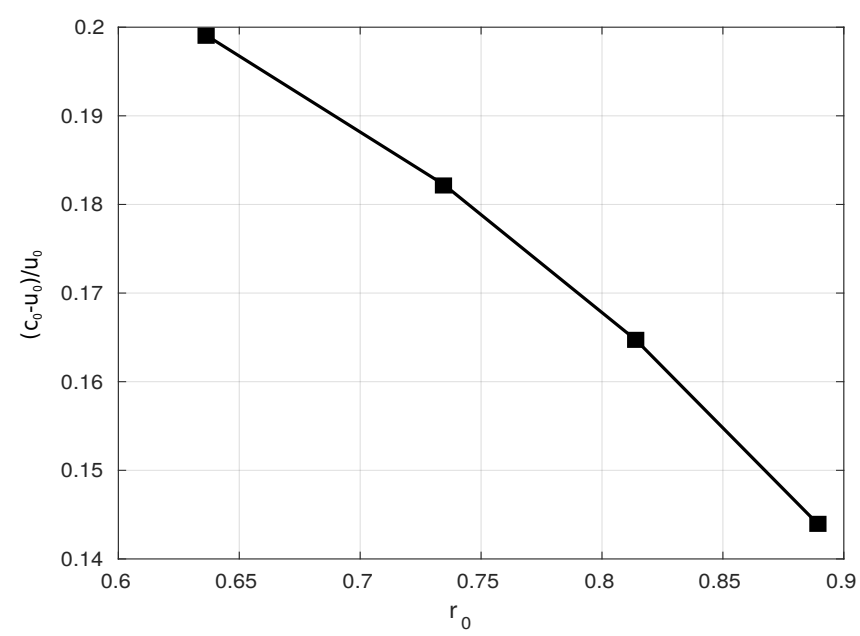

Figura 2.16 - Desviación de la velocidad en el orificio de salida, $u_{0}$, respecto a la velocidad de propagación de ondas en dicho orificio, $c_{0}$, para varios valores del radio del núcleo de aire, $r_{0}$. El resto de parámetros: $L_{s}=2, R_{s}=2, L_{0}=4, \alpha=75$.

\section{Efectos de la geometría del atomizador}

En la figura 2.17(a) se muestra la superficie libre del líquido para varios valores de la longitud del orificio de salida, comparando el espesor de la lámina líquida a la salida con el valor que predice la teoría cuasi-unidireccional (Taylor 1948). En el orificio de salida el flujo se acelera, por lo que, por la ecuación de Bernoulli, la presión disminuye, dando lugar a un aumento del radio del núcleo de aire en esta región. Durante la transición de la superficie libre del líquido desde el valor del radio del núcleo de aire en la cámara al que tiene en el orificio de salida, se genera una componente radial de la velocidad. Si el orificio de salida es suficientemente largo, esta velocidad radial desaparece y el espesor de la lámina líquida en el orificio de salida se aproxima al que predice la teoría cuasi- 
unidireccional. Pero si el orificio no es suficientemente largo, el líquido abandonará el atomizador con una componente radial en su velocidad, lo que no está de acuerdo con la teoría cuasi-unidireccional. En la figura 2.18 se representa la componente radial de la velocidad en la superficie del líquido dentro del atomizador para dos longitudes diferentes del orificio de salida: $L_{0}=2$ y $L_{0}=4$. Esta velocidad radial es positiva en la superficie del líquido, pues el radio del núcleo de aire está creciendo con $x$, pero será negativa cerca de la pared de la cámara, por la presencia de la pared convergente. Se puede observar que dicha velocidad crece según nos aproximamos al orificio de salida (cuyo comienzo se encuentra en $x=0$ ), y luego va disminuyendo a lo largo del mismo. Para el caso con el orificio más corto (cuyo extremo está situado en $x=2$ ), el líquido mantiene a lo largo del mismo un valor apreciable de la velocidad radial, mientras que para el caso del orificio más largo (cuyo estremo está situado en $x=4$ ) esta velocidad prácticamente se anula antes de alcanzar la salida.
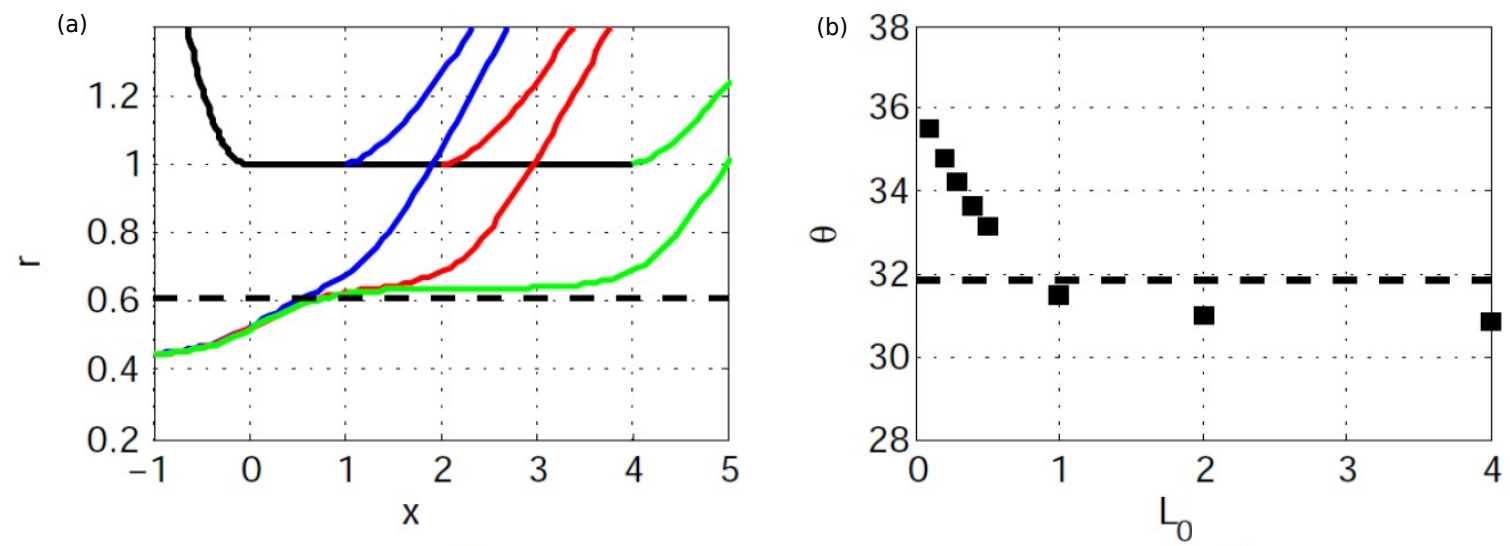

Figura 2.17 - (a) Superficie libre del líquido para diferentes valores de la longitud del orificio de salida: $L_{0}=1$ (azul), $L_{0}=2$ (rojo), $L_{0}=4$ (verde). (b) Variación del semiángulo de la lámina cónica con la longitud del orificio de salida. Resultados numéricos (cuadrados) frente a resultados de la teoría cuasi-unidireccional (Taylor 1948) (líneas discontínuas). El resto de los parámetros: $\Delta=0,785, L_{s}=2, R_{s}=2, \alpha=75$.

Debido a esta velocidad radial, en el sentido positivo de $r$, el ángulo que forma la lámina cónica será mayor para valores pequeños de la longitud del orificio, como se muestra en la figura 2.17(b). Según aumenta la longitud del orificio de salida, el ángulo de la lámina cónica disminuye, de forma cada vez menos marcada, y tiende a aproximarse al valor que predice la teoría cuasi-unidireccional. 


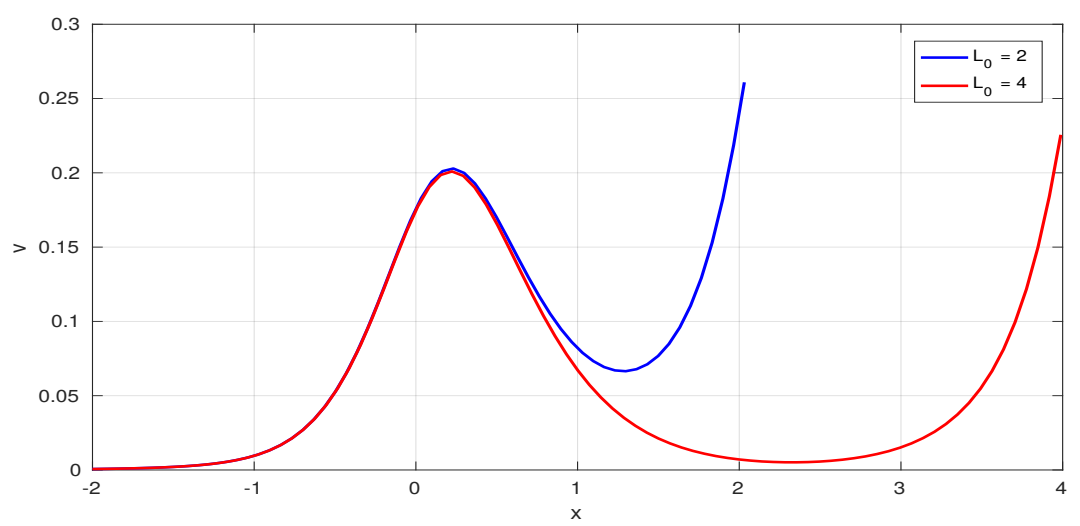

Figura 2.18 - Velocidad radial en la superficie del líquido para varios valores de la longitud del orificio de salida: $L_{0}=2$ (azul), $L_{0}=4$ (rojo). El resto de parámetros: $\Delta=0,785, L_{s}=2, R_{s}=2, \alpha=75$.
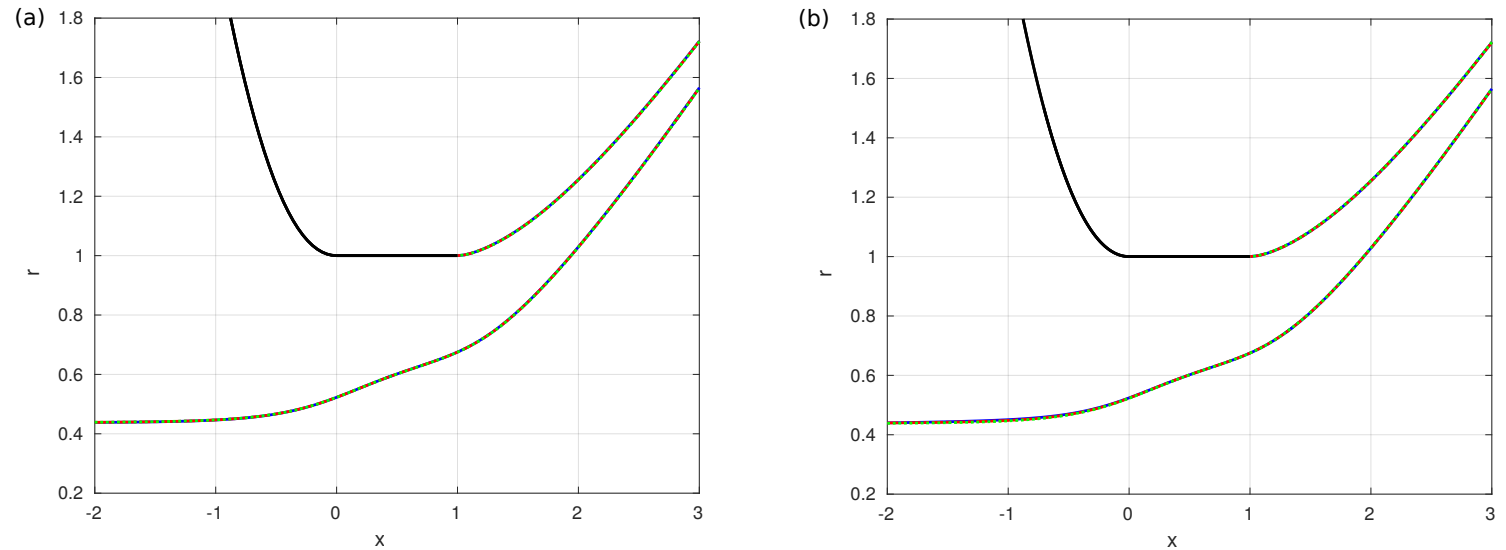

Figura 2.19 - (a) Superficie libre del líquido para diferentes valores de la longitud de la cámara del atomizador: $L_{s}=1$ (azul), $L_{s}=2$ (rojo), $L_{s}=5$ (verde). El resto de los parámetros: $\Delta=0,785, R_{s}=2, L_{0}=1, \alpha=75$. (b) Superficie libre del líquido para diferentes valores del radio de la cámara del atomizador: $R_{s}=1,5$ (azul), $R_{s}=2$ (rojo), $R_{s}=5$ (verde). El resto de los parámetros: $\Delta=0,785, L_{s}=2, L_{0}=1, \alpha=75$.

En la figura 2.19 se muestra la superficie libre del líquido para varios valores de la longitud (a la izquierda) y del radio (a la derecha) de la cámara del atomizador. Puede observarse que las curvas se superponen, por lo que se puede afirmar que dichos parámetros no tienen ningún efecto en las características del flujo, en el marco de la teoría irrotacional.

En la figura 2.20 se muestra la superficie libre del líquido en la región de entrada del orificio de salida para diferentes valores del ángulo de la contracción. La forma que 
adquiere la superficie libre apenas cambia con la variación de dicho ángulo. Sólo se observan pequeñas variaciones en la región antes mencionada: para mayores valores del ángulo de la contracción, el espesor de la película líquida en esta región es ligeramente mayor, debido al mayor giro que tiene que realizar el flujo. Esta pequeña variación no afecta a las características del flujo a la salida del atomizador, pues desaparece rápidamente aguas abajo de la región donde se presentan.

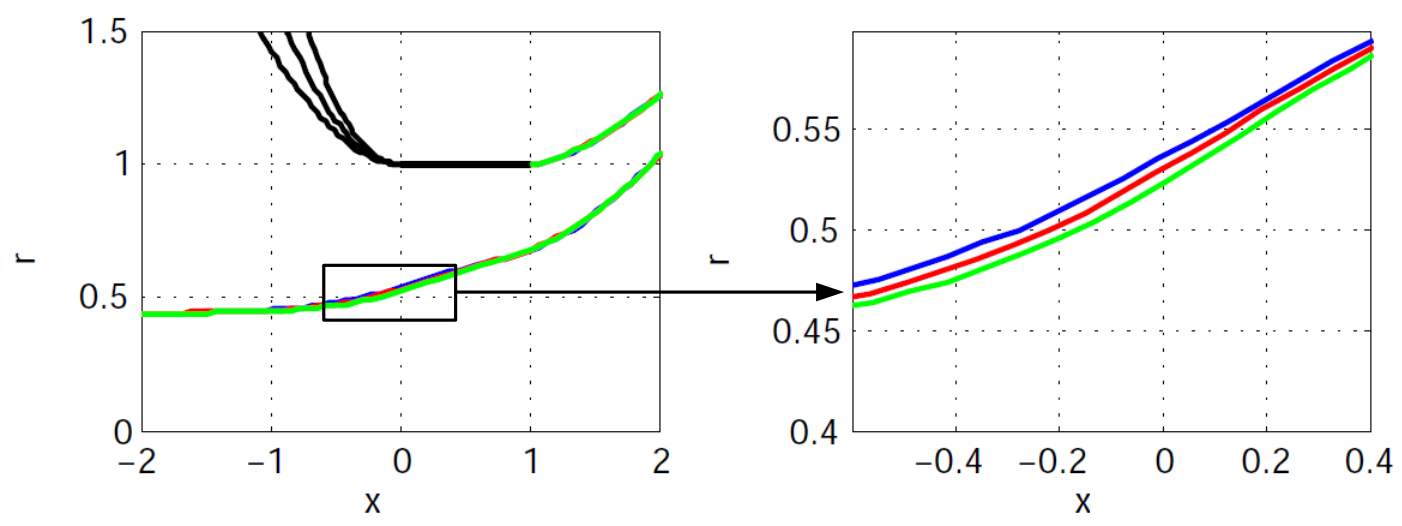

Figura 2.20 - Superficie libre del líquido en la región de entrada al orificio de salida para diferentes valores del ángulo de la contracción: $\alpha=45^{\circ}$ (azul), $\alpha=60^{\circ}$ (rojo), $\alpha=75^{\circ}$ (verde). El resto de los parámetros: $\Delta=0,785, L_{s}=2, R_{s}=2, L_{0}=1$.
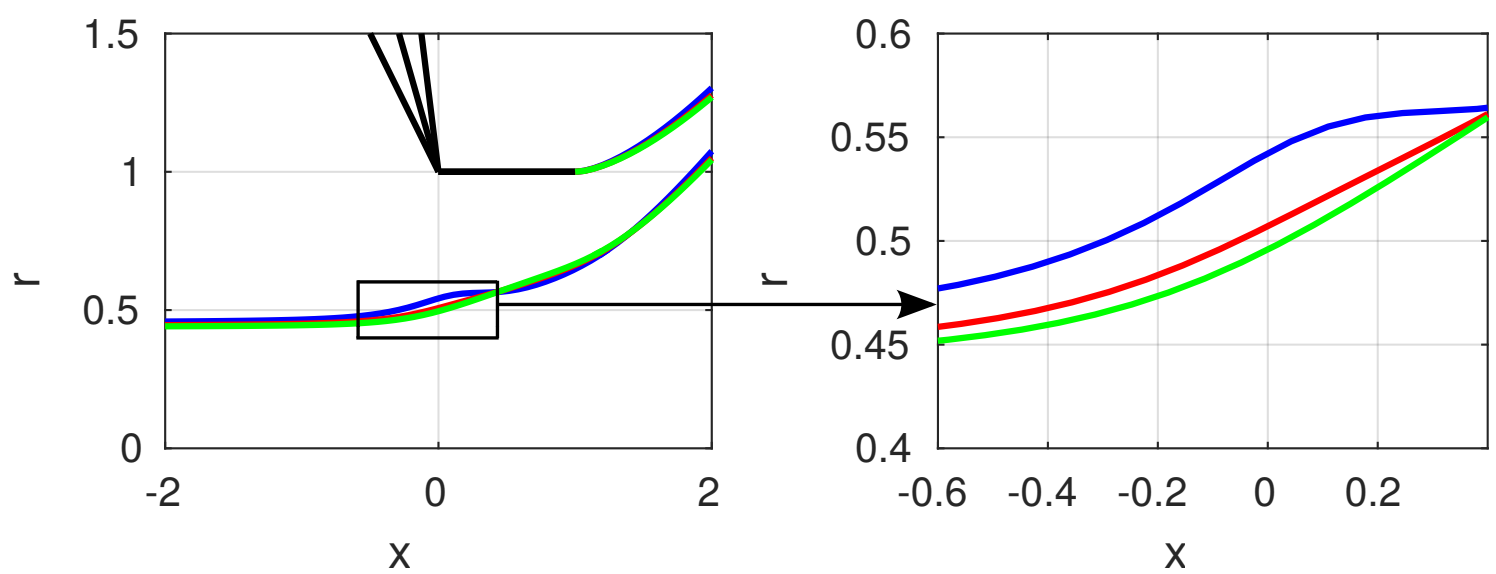

Figura 2.21 - Superficie libre del líquido en la región de entrada al orificio de salida, cuando la transición entre la contracción y dicho orificio es brusca, para diferentes valores del ángulo de la contracción: $\alpha=45^{\circ}$ (azul), $\alpha=60^{\circ}$ (rojo), $\alpha=75^{\circ}$ (verde). El resto de los parámetros: $\Delta=0,785, L_{s}=2, R_{s}=2, L_{0}=1$. 
El efecto anterior puede ser más acusado si la transición entre la contracción y el orificio de salida es más brusca, pues hasta ahora se ha estado usando en el modelo una transición suave. En la figura 2.21 se muestra la superficie libre del líquido en la región de entrada del orificio de salida para diferentes valores del ángulo de la contracción, cuando la transición de ésta al orificio de salida es brusca. Se observa que el efecto del giro del flujo es mayor debido a la mayor componente radial que presenta la velocidad a la entrada del orificio.
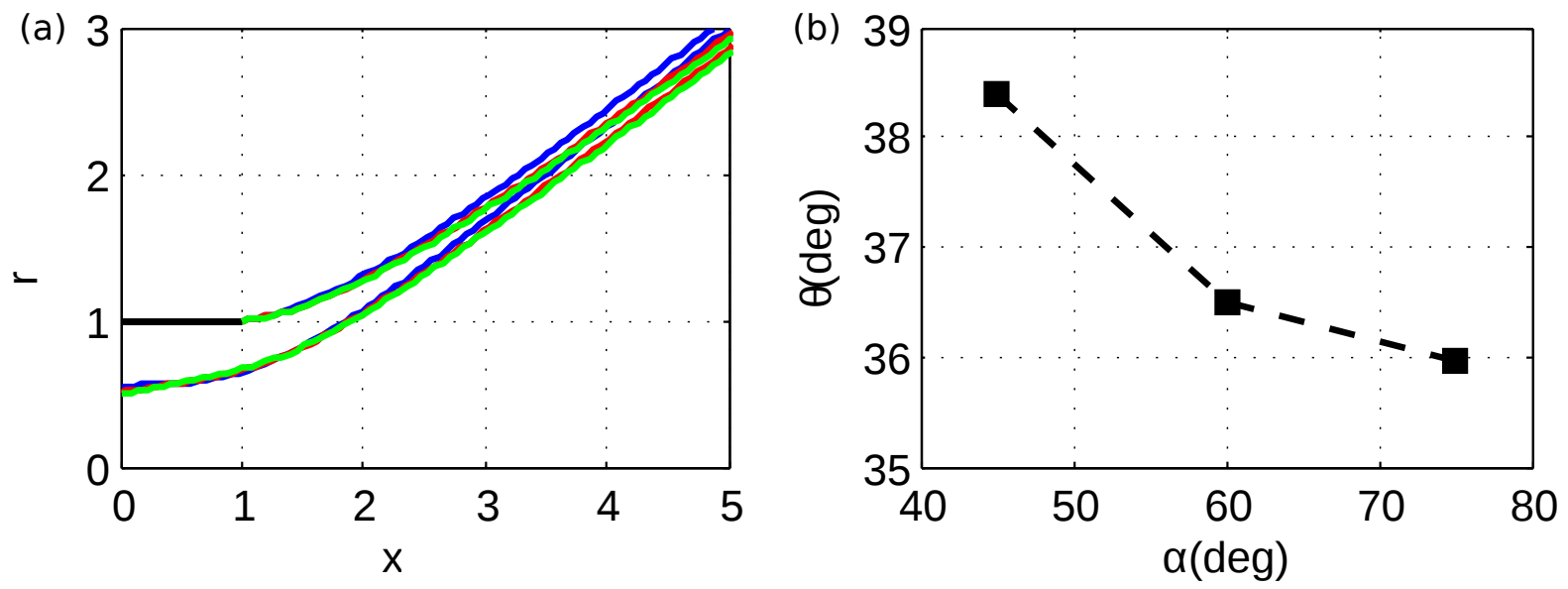

Figura 2.22 - (a) Superficie libre del líquido a la salida del atomizador, cuando la transición de la contracción al orificio de salida es brusca, para diferentes valores del ángulo de la contracción: $\alpha=45^{\circ}$ (azul), $\alpha=60^{\circ}$ (rojo), $\alpha=75^{\circ}$ (verde). (b) Variación del semiángulo de la lámina cónica $\theta$ con el ángulo de la contracción $\alpha$. El resto de los parámetros: $\Delta=0,785, L_{s}=2, R_{s}=2, L_{0}=1$.

El giro del flujo al pasar de la contracción al orificio de salida genera una componente radial negativa (hacia el eje) de la velocidad en la zona próxima a la pared de la cámara, que será mayor cuanto mayor sea el ángulo de la contracción. Esta velocidad radial, cuando la transición entre la contracción y el orificio de salida es brusca, puede seguir presente a la salida del orificio, afectando al ángulo del cono que forma la lámina líquida, como se muestra en la figura 2.22. En la misma puede observarse que el ángulo de la lámina cónica disminuye cuando aumenta el ángulo de la contracción, debido a la mayor componente radial de velocidad. 


\subsection{Propagación de ondas en la superficie del núcleo de aire}

El flujo en el interior de un atomizador de presión con giro es un ambiente idóneo para la propagación de ondas, pues el mismo presenta una superficie libre (la superficie del núcleo de aire), y una fuerza másica aparente (la debida a la aceleración centrípeta). Estudiaremos la propagación de ondas en el interior de la cámara del atomizador y en el orificio de salida, así como la transmisión y reflexión de las mismas en la zona de transición entre ambas regiones, usando simplificaciones derivadas de la diferencia de escalas típica de estos dispositivos.

Empezaremos admitiendo que el radio de la pared de la cámara, $R_{s}$, es mucho mayor que el radio del núcleo de aire $r_{s_{s t}}$ en el interior de la misma en régimen estacionario, $R_{s} \gg r_{s_{s t}}$. Esto permite suponer que la pared se encuentra en el infinito y despreciar la componente axial de la velocidad del líquido en la cámara frente a su componente azimutal. Cerca del orificio de salida, sin embargo, el radio de la pared $R_{0}$ es del orden del radio del núcleo de aire $r_{0_{s t}}$ en el interior del mismo en régimen estacionario, $R_{0} \sim r_{0_{s t}}$, y la velocidad axial del líquido $u_{0_{s t}}$ en régimen estacionario no se puede despreciar. Debido a ello, el radio del núcleo de aire cambia a través de la región de transición. El valor de dicho radio en el orificio de salida puede obtenerse en función de su valor en la cámara y de la velocidad axial del líquido en el orificio por medio de la ecuación de Bernoulli:

$$
\frac{\Gamma^{2}}{4 \pi^{2} r_{s_{s t}}^{2}}=u_{0_{s t}}^{2}+\frac{\Gamma^{2}}{4 \pi^{2} r_{0_{s t}}^{2}}
$$

Introducimos pequeñas perturbaciones en el radio del núcleo de aire y en el potencial de velocidades, que designaremos como $\eta_{s} \mathrm{y} \varphi_{s_{p}}$ en la cámara, y $\eta_{0} \mathrm{y} \varphi_{0_{p}}$ en la región en torno al orificio de salida, tales que $\left(\eta_{s}, \eta_{0}\right) \ll\left(r_{s}, r_{0}\right)$ y $\left(\left|\nabla \varphi_{s_{p}}\right|,\left|\nabla \varphi_{0_{p}}\right|\right) \ll\left[\Gamma /\left(2 \pi r_{s}\right), \Gamma /\left(2 \pi r_{0}\right)\right]$. Los valores del radio del núcleo de aire perturbado son $r_{s}=r_{s_{s t}}+\eta_{s}$ y $r_{0}=r_{0_{s t}}+\eta_{0}$.

El problema a resolver es el problema de frontera libre definido por la ecuación de Laplace (2.8), con las correspondientes condiciones de contorno (2.9) y (2.10). De momento supondremos que la cámara es infinitamentemente larga, para no preocuparnos por las condiciones de contorno en sus extremos, que se analizarán posteriormente. De esta forma tenemos, en el interior de la cámara:

$$
\begin{gathered}
\frac{\partial^{2} \varphi_{s_{p}}}{\partial x^{2}}+\frac{1}{r} \frac{\partial}{\partial r}\left(r \frac{\partial \varphi_{s_{p}}}{\partial r}\right)=0 \\
\frac{\partial r_{s}}{\partial t}+\frac{\partial \varphi_{s_{p}}}{\partial x} \frac{\partial r_{s}}{\partial x}-\frac{\partial \varphi_{s_{p}}}{\partial r}=0 \quad \text { en } \quad r=r_{s}
\end{gathered}
$$




$$
\begin{gathered}
\frac{\partial \varphi_{s_{p}}}{\partial t}+\frac{1}{2}\left(\frac{\partial \varphi_{s_{p}}}{\partial x}\right)^{2}+\frac{1}{2}\left(\frac{\partial \varphi_{s_{p}}}{\partial r}\right)^{2}+\frac{\Gamma^{2}}{8 \pi^{2} r_{s}^{2}}+\frac{p_{a}}{\rho}=C_{s}(t) \text { en } r=r_{s} \\
\nabla \varphi_{s_{p}} \rightarrow 0 \text { en } r \rightarrow \infty
\end{gathered}
$$

mientras que en la región en torno al orificio de salida se tiene:

$$
\begin{gathered}
\frac{\partial^{2} \varphi_{0_{p}}}{\partial x^{2}}+\frac{1}{r} \frac{\partial}{\partial r}\left(r \frac{\partial \varphi_{0_{p}}}{\partial r}\right)=0 \\
\frac{\partial r_{0}}{\partial t}+\left(u_{0_{s t}}+\frac{\partial \varphi_{0_{p}}}{\partial x}\right) \frac{\partial r_{0}}{\partial x}-\frac{\partial \varphi_{0_{p}}}{\partial r}=0 \quad \text { en } \quad r=r_{0} \\
\frac{\partial \varphi_{0_{p}}}{\partial t}+\frac{1}{2}\left(u_{0_{s t}}+\frac{\partial \varphi_{0_{p}}}{\partial x}\right)^{2}+\frac{1}{2}\left(\frac{\partial \varphi_{0_{p}}}{\partial r}\right)^{2}+\frac{\Gamma^{2}}{8 \pi^{2} r_{0}^{2}}+\frac{p_{a}}{\rho}=C_{0}(t) \quad \text { en } \quad r=r_{0} \\
\frac{\partial \varphi_{0_{p}}}{\partial r}=0 \quad \text { en } r=R_{0}
\end{gathered}
$$

El desarrollo completo de este análisis se incluye en el Apéndice A. Escalando las variables espaciales con $R_{0}$, las velocidades con $\Gamma /\left(2 \pi R_{0}\right)$, las presiones con $\rho \Gamma^{2} /\left(4 \pi^{2} R_{0}^{2}\right)$ y el tiempo con $2 \pi R_{0}^{2} / \Gamma$, linealizando las ecuaciones y expresando la solución de la siguiente forma:

$$
\begin{array}{cll}
\varphi_{s_{p}}=\hat{\varphi}_{s}(r) e^{-i k_{s} x+i \omega t}+c c \quad ; \quad & \varphi_{0_{p}}=\hat{\varphi}_{0}(r) e^{-i k_{0} x+i \omega t}+c c \\
\eta_{s}=\hat{\eta}_{s} e^{-i k_{s} x+i \omega t}+c c \quad ; \quad & \eta_{0}=\hat{\eta}_{0} e^{-i k_{0} x+i \omega t}+c c
\end{array}
$$

donde $c c$ indica complejo conjugado, y $k_{s}$ y $k_{0}$ son los números de onda asociados a una cierta frecuencia $\omega$ mediante unas relaciones de dispersión que se calcularán más adelante, se llega, tras eliminar las variables $\eta_{s}$ y $\eta_{0}$, al siguiente problema para el flujo en el interior de la cámara:

$$
\begin{gathered}
\frac{1}{r} \frac{\mathrm{d}}{\mathrm{d} r}\left(r \frac{\mathrm{d} \hat{\varphi}_{s}}{\mathrm{~d} r}\right)-k_{s}^{2} \hat{\varphi}_{s}=0 \\
-\omega^{2} \hat{\varphi}_{s}-\frac{1}{r_{s_{s t}}^{3}} \frac{\mathrm{d} \hat{\varphi}_{s}}{\mathrm{~d} r}=0 \quad \text { en } \quad r=r_{s_{s t}} \\
\hat{\varphi}_{s} \rightarrow 0 \quad \text { en } \quad r \rightarrow \infty
\end{gathered}
$$

mientras que en la región en torno al orificio se tiene:

$$
\begin{gathered}
\frac{1}{r} \frac{\mathrm{d}}{\mathrm{d} r}\left(r \frac{\mathrm{d} \hat{\varphi}_{0}}{\mathrm{~d} r}\right)-k_{0}^{2} \hat{\varphi}_{0}=0 \\
-\left(\omega-u_{0_{s t}} k_{0}\right)^{2} \hat{\varphi}_{0}-\frac{1}{r_{0_{s t}}^{3}} \frac{\mathrm{d} \hat{\varphi}_{0}}{\mathrm{~d} r}=0 \quad \text { en } \quad r=r_{0_{s t}} \\
\frac{\mathrm{d} \hat{\varphi}_{0}}{\mathrm{~d} r}=0 \quad \text { en } \quad r=1
\end{gathered}
$$


Las soluciones generales de las ecuaciones (2.98) y (2.101) son:

$$
\begin{aligned}
& \hat{\varphi}_{s}=a_{s} K_{0}\left(k_{s} r\right)+b_{s} I_{0}\left(k_{s} r\right) \\
& \hat{\varphi}_{0}=a_{0} K_{0}\left(k_{0} r\right)+b_{0} I_{0}\left(k_{0} r\right)
\end{aligned}
$$

donde las funciones $I_{0}$ y $K_{0}$ son las funciones de Bessel modificadas de orden cero de primera y segunda especie, respectivamente, y las constantes $a_{s}, b_{s}, a_{0}$ y $b_{0}$ se determinarán imponiendo las condiciones de contorno correspondientes. Las expresiones $\hat{\eta}_{s}$ y $\hat{\eta}_{0}$ quedan:

$$
\begin{gathered}
\hat{\eta}_{s}=i \omega r_{s_{s t}}^{3} \hat{\varphi}_{s} \\
\hat{\eta}_{0}=i\left(\omega-u_{0_{s t}} k_{0}\right) r_{0_{s t}}^{3} \hat{\varphi}_{0}
\end{gathered}
$$

\subsubsection{Ondas en la cámara}

El potencial de velocidades en la cámara es (ver Apéndice A):

$$
\hat{\varphi}_{s}=a_{s} K_{0}\left(k_{s} r\right)
$$

mientras que la relación de dispersión es:

$$
\omega^{2}=-\frac{k_{s}}{r_{s_{s t}}^{3}} \frac{K_{0}^{\prime}\left(k_{s} r_{s_{s t}}\right)}{K_{0}\left(k_{s} r_{s_{s t}}\right)}
$$

En el caso de longitudes de onda grandes frente al radio del núcleo de aire, $k_{s} r_{s_{s t}} \ll 1$, la relación de dispersión se reduce a:

$$
\omega^{2}\left[-\ln \left(\frac{k_{s} r_{s_{s t}}}{2}\right)-\gamma\right]=\frac{1}{r_{s_{s t}}^{4}}
$$

donde $\gamma$ es la constante de Euler-Mascheroni, de valor aproximado $\gamma \approx 0,5772$. De aquí se obtiene la siguiente velocidad de fase de las ondas:

$$
c_{s}=\frac{\omega}{k_{s}}=\frac{ \pm 1}{k_{s} r_{s_{s t}}^{2}}\left[-\ln \left(\frac{k_{s} r_{s_{s t}}}{2}\right)-\gamma\right]^{-1 / 2}
$$

mientra que la velocidad de grupo es:

$$
c_{g_{s}}=\frac{\mathrm{d} \omega}{\mathrm{d} k_{s}}=\frac{ \pm 1}{2 k_{s} r_{s_{s t}}^{2}}\left[-\ln \left(\frac{k_{s} r_{s_{s t}}}{2}\right)-\gamma\right]^{-3 / 2}
$$

Obsérvese que la velocidad de fase de las ondas dada por la expresión (2.111) varía con la longitud de onda, luego el sistema es dispersivo, incluso en este límite de onda larga. Los 
modos asociados a diferentes números de onda $k_{s}$ se propagarán a diferente velocidad, lo que dará lugar a la deformación de la onda. En concreto, la velocidad será menor cuanto mayor sea la longitud de onda. El resultado obtenido en (2.111) es específico de este problema, y diferente del problema de las ondas gravitatorias en el agua, donde la velocidad de las ondas es constante en el límite de aguas someras (longitud de onda grande frente a la profundidad).

En el caso contrario, cuando $k_{s} r_{s_{s t}} \gg 1$, la relación de dispersión (2.109) puede expresarse como:

$$
\omega^{2}=\frac{k_{s}}{r_{s_{s t}}^{3}}
$$

y la velocidad de fase de las ondas:

$$
c_{s}=\frac{\omega}{k_{s}}=\frac{ \pm 1}{\sqrt{k_{s} r_{s t}^{3}}}
$$

mientra que la velocidad de grupo es:

$$
c_{g_{s}}=\frac{\mathrm{d} \omega}{\mathrm{d} k_{s}}=\frac{ \pm 1}{2 \sqrt{k_{s} r_{s_{s t}}^{3}}}
$$

En este caso, la velocidad de fase de las ondas dada por la expresión (2.114) también varía con la longitud de onda, luego el sistema también es dispersivo, pero esta vez es $c \sim k^{-1 / 2}$, por lo que el problema es análogo al de las ondas gravitatorias en aguas profundas.

\subsubsection{Ondas cerca del orificio de salida}

El potencial de velocidades cerca del orificio de salida es (ver Apéndice A):

$$
\hat{\varphi}_{0}=a_{0}\left(\frac{K_{0}\left(k_{0} r\right)}{K_{0}^{\prime}\left(k_{0}\right)}-\frac{I_{0}\left(k_{0} r\right)}{I_{0}^{\prime}\left(k_{0}\right)}\right)
$$

mientras que la relación de dispersión es:

$$
\left(\omega-u_{0_{s t}} k_{0}\right)^{2}=-\frac{k_{0}}{r_{0_{s t}}^{3}} \frac{K_{0}^{\prime}\left(k_{0} r_{0_{s t}}\right) I_{0}^{\prime}\left(k_{0}\right)-K_{0}^{\prime}\left(k_{0}\right) I_{0}^{\prime}\left(k_{0} r_{0_{s t}}\right)}{K_{0}\left(k_{0} r_{0_{s t}}\right) I_{0}^{\prime}\left(k_{0}\right)-K_{0}^{\prime}\left(k_{0}\right) I_{0}\left(k_{0} r_{0_{s t}}\right)}
$$

En el límite $\left(k_{0} r_{0_{s t}}, k_{0}\right) \ll 1$, la relación de dispersión se reduce a:

$$
\left(\omega-u_{0_{s t}} k_{0}\right)^{2}=k_{0}^{2} \frac{1-r_{0_{s t}}^{2}}{2 r_{0_{s t}}^{4}}
$$

De la relación de dispersión (2.118) se obtiene que la velocidad de las ondas relativa a la cámara es igual a la velocidad de grupo, y de valor:

$$
c_{0}=c_{g_{0}}=u_{0_{s t}} \pm \frac{1}{r_{0_{s t}}^{2}} \sqrt{\frac{1}{2}\left(1-r_{0_{s t}}^{2}\right)}
$$


mientras que la velocidad de las ondas relativa al líquido es:

$$
\frac{\omega-u_{0_{s t}} k_{0}}{k_{0}}=\frac{ \pm 1}{r_{0_{s t}}^{2}} \sqrt{\frac{1}{2}\left(1-r_{0_{s t}}^{2}\right)}
$$

La velocidad de las ondas de la expresión (2.119) es independiente de la longitud de onda, luego se trata de ondas no dispersivas, las cuales se propagan sin deformarse. El resultado es análogo al del problema de propagación de ondas en aguas poco profundas, donde la velocidad de propagación es $c=\sqrt{g h}$, siendo $h$ la profundidad del líquido.

En el caso en que $k_{0} r_{0_{s t}} \ll 1$, pero $k_{0} \sim O(1)$, podemos despreciar el segundo sumando del numerador de la expresión de la relación de dispersión (2.117), por lo que la misma se reduce a:

$$
\left(\omega-u_{0_{s t}} k_{0}\right)^{2}=\frac{1 / r_{0_{s t}}^{4}}{-\left[\ln \left(\frac{k_{0} r_{0 s t}}{2}\right)+\gamma\right]-K_{0}^{\prime}\left(k_{0}\right) / I_{0}^{\prime}\left(k_{0}\right)}
$$

En la figura 2.23 se representan las velocidades de fase y de grupo de las ondas en función del número de onda, para varios valores del radio adimensional del núcleo de aire. Puede observarse que la velocidad es mayor cuanto menor es el número de onda, esto es, las ondas de mayor longitud de onda se propagan más rápido. En dicha figura también puede verse que para valores pequeños del número de onda ambas velocidades coinciden, como se había visto anteriormente en el límite $\left(k_{0} r_{0_{s t}}, k_{0}\right) \ll 1$. Además, las velocidades son menores cuanto mayor sea el radio del núcleo de aire.
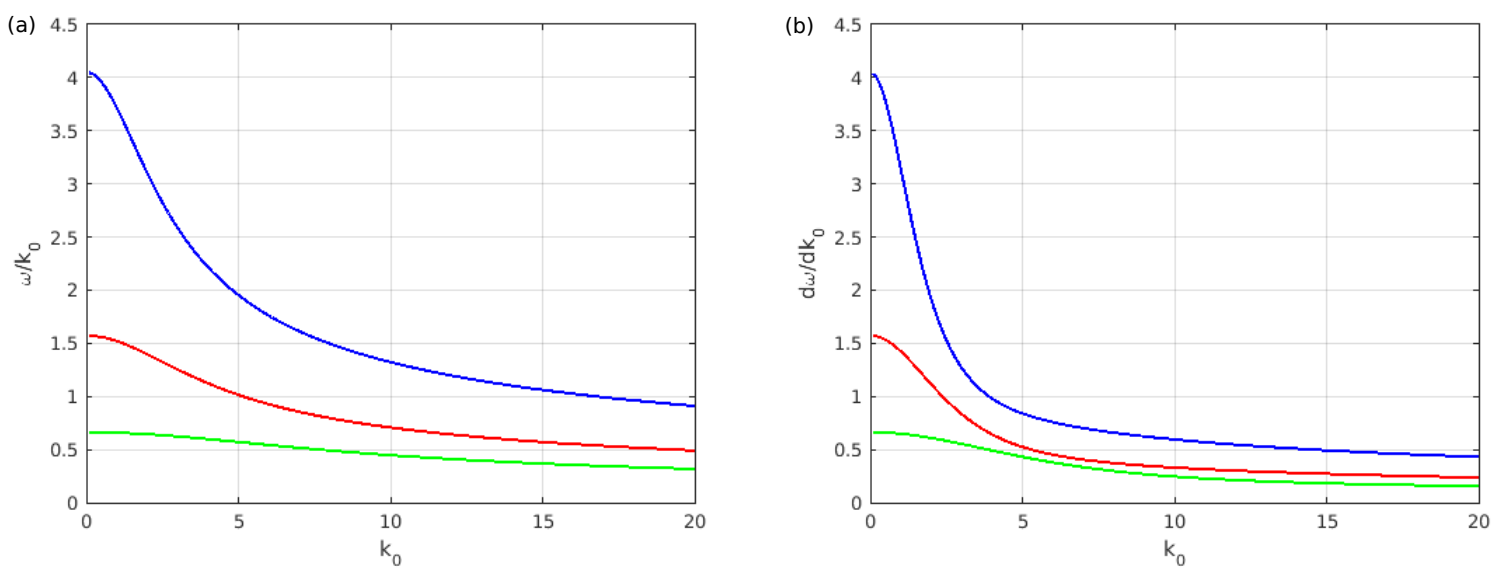

Figura 2.23 - (a) Velocidad de fase y (b) velocidad de grupo de las ondas en función del número de onda, para varios valores del radio adimensional del núcleo de aire: $r_{0_{s t}}=0,4$ (azul), $r_{0_{s t}}=0,6$ (rojo) y $r_{0_{s t}}=0,8$ (verde). 


\subsubsection{Reflexión y transmisión de ondas en la región de transición entre la cámara y el orificio de salida}

Entre la cámara y el orificio de salida se produce un cambio de sección que, para simplificar el análisis, consideraremos compacto. Un cambio de sección compacto es aquel en el que el tamaño característico de la región de transición donde la sección cambia es pequeño comparado con la longitud de onda característica de la onda incidente, o que la frecuencia de las ondas es pequeña comparada con la relación entre la velocidad de onda característica y la longitud característica de la región de transición. Para ondas de sonido en un tubo $u$ ondas de gravedad en aguas poco profundas en un canal, esto implica que el flujo es cuasi-estacionario en la región de transición y la variación de presión y el caudal asociados a las ondas son continuos a través de la transición (Lighthill 1978). Consideraremos que dicha región de transición está localizada en $x=0$.

Estos resultados serían aplicables a las ondas en la cámara de un atomizador de presión con giro cuando $\left(k_{s} r_{s_{s t}}, k_{s} R_{s}, k_{0} r_{0_{s t}}, k_{0}\right) \ll 1$, donde $k_{s}$ y $k_{0}$ son los números de onda asociados a una cierta frecuencia $\omega$ mediante las relaciones de dispersión (2.109) y (2.117) en cada una de las dos zonas. Aunque la condición $k_{s} R_{s} \ll 1$ es contraria a lo supuesto al comienzo de esta sección, y sólo tendría sentido para una cámara de longitud grande frente a $R_{s}$, que no es realista, discutiremos brevemente este caso.

De la expresión (2.116), y teniendo en cuenta las aproximaciones de las funciones de Bessel modificadas en el límite $\left(k_{s} r_{s_{s t}}, k_{s} R_{s}, k_{0} r_{0_{s t}}, k_{0}\right) \ll 1$, se obtienen las siguientes expresiones para las funciones $\hat{\varphi}_{s} \mathrm{y} \hat{\varphi}_{0}$ en los potenciales de velocidades en la cámara y cerca del orificio:

$$
\hat{\varphi}_{s}=-\frac{2 a_{s}}{k_{s} R_{s}} \quad ; \quad \hat{\varphi}_{0}=-\frac{2 a_{0}}{k_{0}}
$$

mientras que las funciones $\hat{u}_{s}=-i k_{s} \hat{\varphi}_{s}$ y $\hat{u}_{0}=-i k_{0} \hat{\varphi}_{0}$, que aparecerían en expresiones análogas a (2.5) para la perturbación de la componente axial de la velocidad del líquido, que es constante en cada sección transversal, son:

$$
\hat{u}_{s}=-i \frac{2 a_{s}}{R_{s}} \quad ; \quad \hat{u}_{0}=-i 2 a_{0}
$$

Introduciendo (2.122) en (2.106) y (2.107) se obtiene, para la deformación de la superficie libre:

$$
\hat{\eta}_{s}=-i \frac{2 a_{s} \omega r_{s_{s t}}^{3}}{k_{s} R_{s}} ; \quad \hat{\eta}_{0}=-i \frac{2 a_{0}\left(\omega-u_{0_{s t}} k_{0}\right) r_{0_{s t}}^{3}}{k_{0}}
$$

Llamaremos $p_{s_{p}}$ y $p_{0_{p}}$ a las perturbaciones de la presión en la cámara y en la región en torno al orificio de salida, respectivamente, debidas a las ondas. Estas perturbaciones 
están relacionadas con $\varphi_{s_{p}}$ y $\varphi_{0_{p}}$ mediante la ecuación de Euler-Bernoulli linealizada en el interior del líquido, que da:

$$
p_{s_{p}}=-\frac{\partial \varphi_{s_{p}}}{\partial t}
$$

en la cámara, y

$$
p_{0_{p}}=-\frac{\partial \varphi_{0_{p}}}{\partial t}-u_{0_{s t}} \frac{\partial \varphi_{0_{p}}}{\partial x}
$$

en la región del orificio. La continuidad del exceso de presión debido a las ondas en la región de transición implica que la suma del exceso de presión de las ondas incidente y reflejada, de amplitudes $a_{s}$ y $d_{s}$ respectivamente, sea igual al de la onda transmitida, de amplitud $a_{0}$. Teniendo en cuenta las expresiones (2.125) y (2.126), esto se traduce en:

$$
i \frac{2 \omega}{k_{s} R_{s}}\left(a_{s} e^{i \omega t}+d_{s} e^{i \omega t}\right)=i \frac{2}{k_{0}}\left(\omega-k_{0} u_{0_{s t}}\right) a_{0} e^{i \omega t}
$$

De la conservación del caudal, $A_{s} u_{s}=A_{0} u_{0}$, donde $A_{s}$ y $A_{0}$ son las áreas de una sección transversal del volumen ocupado por el líquido en la cámara y en el orificio de salida, respectivamente, se obtiene la siguiente ecuación:

$$
-\pi\left(R_{s}^{2}-r_{s_{s t}}^{2}\right) \frac{2 i}{R_{s}}\left(a_{s} e^{i \omega t}-d_{s} e^{i \omega t}\right)=-\pi\left(1-r_{0_{s t}}^{2}\right) 2 i a_{0} e^{i \omega t}
$$

Las expresiones (2.127) y (2.128) se reducen al siguiente sistema de dos ecuaciones con dos incógnitas, las amplitudes de la onda reflejada, $d_{s}$, y de la onda transmitida, $a_{0}$ :

$$
\begin{gathered}
a_{s}+d_{s}=\frac{k_{s} R_{s}}{k_{0}} \frac{\omega-k_{0} u_{0_{s t}}}{\omega} a_{0} \\
a_{s}-d_{s}=R_{s} \frac{1-r_{0_{s t}}^{2}}{R_{s}^{2}-r_{s_{s t}}^{2}} a_{0}
\end{gathered}
$$

Del sistema formado por (2.129) y (2.130) se obtiene:

$$
\begin{gathered}
a_{0}=a_{s} \frac{2}{R_{s}} \frac{R_{s}^{2}-r_{s_{s t}}^{2}}{1-r_{0_{s t}}^{2}}\left[\frac{k_{s}}{k_{0}} \frac{R_{s}^{2}-r_{s_{s t}}^{2}}{1-r_{0_{s t}}^{2}} \frac{\omega-k_{0} u_{0_{s t}}}{\omega}+1\right]^{-1} \\
d_{s}=a_{s}\left[\frac{k_{s}}{k_{0}} \frac{R_{s}^{2}-r_{s_{s t}}^{2}}{1-r_{0_{s t}}^{2}} \frac{\omega-k_{0} u_{0_{s t}}}{\omega}-1\right]\left[\frac{k_{s}}{k_{0}} \frac{R_{s}^{2}-r_{s_{s t}}^{2}}{1-r_{0_{s t}}^{2}} \frac{\omega-k_{0} u_{0_{s t}}}{\omega}+1\right]^{-1}
\end{gathered}
$$

Cuando $k_{s} r_{s_{s t}} \ll 1$ con $k_{s} R_{s}$ de orden unidad o mayor en la cámara y $r_{0_{s t}} \sim 1$ en el orificio de salida, la región de transición actúa como una pared cerrada excepto a una distancia de orden $O\left(r_{0_{s t}}\right)$ del orificio. La amplitud de la onda reflejada es igual a la amplitud de la onda incidente en una primera aproximación, mientras que el exceso de presión alrededor del orificio y la deformación de la superficie libre son el doble de las de 
la onda incidente. Teniendo en cuenta esto y la expresión (2.106), la deformación de la superficie es:

$$
\hat{\eta}_{s}=2 i \omega r_{s t}^{3} \hat{\varphi}_{s}\left(r_{s_{s t}}\right)
$$

donde la función $\hat{\varphi}_{s}\left(r_{s_{s t}}\right)$ en la expresión del potencial viene dada por (2.116):

$$
\hat{\varphi}_{s}\left(r_{s_{s t}}\right)=a_{s}\left(\frac{K_{0}\left(k_{s} r_{s_{s t}}\right)}{K_{0}^{\prime}\left(k_{s} R_{s}\right)}-\frac{I_{0}\left(k_{s} r_{s_{s t}}\right)}{I_{0}^{\prime}\left(k_{s} R_{s}\right)}\right) \approx-a_{s}\left(\frac{\ln \left(\frac{k_{s} r_{s s t}}{2}\right)+\gamma}{K_{0}^{\prime}\left(k_{s} R_{s}\right)}+\frac{1}{I_{0}^{\prime}\left(k_{s} R_{s}\right)}\right)
$$

La deformación ha de ser continua a través de la región de transición, $\hat{\eta}_{s}=\hat{\eta}_{0}$, luego, de (2.133) y (2.107):

$$
2 i \omega r_{s_{s t}}^{3} \hat{\varphi}_{s}\left(r_{s_{s t}}\right)=i\left(\omega-U_{0} k_{0}\right) r_{0_{s t}}^{3} \hat{\varphi}_{0}\left(r_{0_{s t}}\right)
$$

Así pues, la amplitud de la onda transmitida será:

$$
a_{0}=a_{s} k_{0} \frac{\omega}{\omega-u_{0 s t} k_{0}} \frac{r_{s_{s t}}^{3}}{r_{0_{s t}}^{3}}\left(\frac{\ln \left(\frac{k_{s} r_{s t}}{2}\right)+\gamma}{K_{0}^{\prime}\left(k_{s} R_{s}\right)}+\frac{1}{I_{0}^{\prime}\left(k_{s} R_{s}\right)}\right)
$$

La onda incidente cederá una parte de su energía a la onda transmitida, por lo que la onda reflejada tendrá ligeramente menos energía que la onda incidente. Esta onda reflejada se propagará hacia el final de la cámara y regresará hacia el orificio como onda incidente, pero de menor energía que la anterior. Parte de su energía la volverá a ceder a la onda transmitida, por lo que se reflejará con una energía menor. Así pues, la energía de la onda de la cámara se irá disipando.

Para calcular la amplitud de la onda reflejada se aplicará la conservación de la energía. Esto es, de acuerdo con Lighthill (1978):

$$
\int_{r_{s_{s t}}+\eta_{s}}^{R_{s}} \overline{p_{s_{p}} u_{s_{p}}} 2 \pi r \mathrm{~d} r=\int_{r_{0_{s t}}+\eta_{0}}^{1} \overline{p_{0_{p}}\left(u_{0_{s t}}+u_{0_{p}}\right)} 2 \pi r \mathrm{~d} r
$$

donde la línea sobre las variables indica valor promedio, y $u_{s_{p}}$ y $u_{0_{p}}$ son las perturbaciones en la velocidad axial del líquido en la cámara y cerca del orificio de salida, respectivamente. El desarrollo de este análisis se incluye en el Apéndice B. De la relación (2.136) se obtiene la siguiente expresión para la disipación:

$$
\begin{aligned}
& 1-\frac{\left|d_{s}\right|^{2}}{\left|a_{s}\right|^{2}}= \\
& \quad \frac{2\left(\omega-u_{0 s t} k_{0}\right)}{\omega k_{s} k_{0}} \frac{\left|a_{0}\right|^{2}}{\left|a_{s}\right|^{2}}\left(1-r_{0_{s t}}^{2}+\frac{2 r_{0_{s t}}^{4} u_{0_{s t}}\left(\omega-u_{0 s t} k_{0}\right)}{k_{0}}\right)\left(\int_{r_{s t}}^{R_{s}} F_{s}^{2}(r) r \mathrm{~d} r\right)^{-1}
\end{aligned}
$$


2.5. Propagación de ondas en la superficie del núcleo de aire en una cámara de sección lentamente variable

La pérdida de energía en cada reflexión se puede asimilar a una parte imaginaria de $\omega$. Teniendo en cuenta que las ondas se propagan en la cámara a una velocidad $c_{s}=\omega / k_{s}$, el tiempo que tarda la onda en viajar desde el orificio a la pared opuesta de la cámara y volver es $\Delta t=2 L_{s} k_{s} / \omega$, donde $L_{s}$ es la longitud de la cámara, escalada con el radio del orificio $R_{0}$. Así pues, esta parte imaginaria de $\omega$ será igual a la pérdida de energía por cada periodo, esto es:

$$
\omega_{\text {dis }}=\frac{1}{2}\left(1-\frac{\left|d_{s}\right|^{2}}{\left|a_{s}\right|^{2}}\right) \frac{1}{\Delta t}
$$

En la figura 2.24 se representa $\omega_{\text {dis }}$ en función del número de onda para varios valores de la velocidad en el orificio y del radio del núcleo de aire en la cámara. Puede observarse que el ritmo de disipación disminuye cuando aumentan $u_{0_{s t}} \mathrm{y} r_{s_{s t}}$.
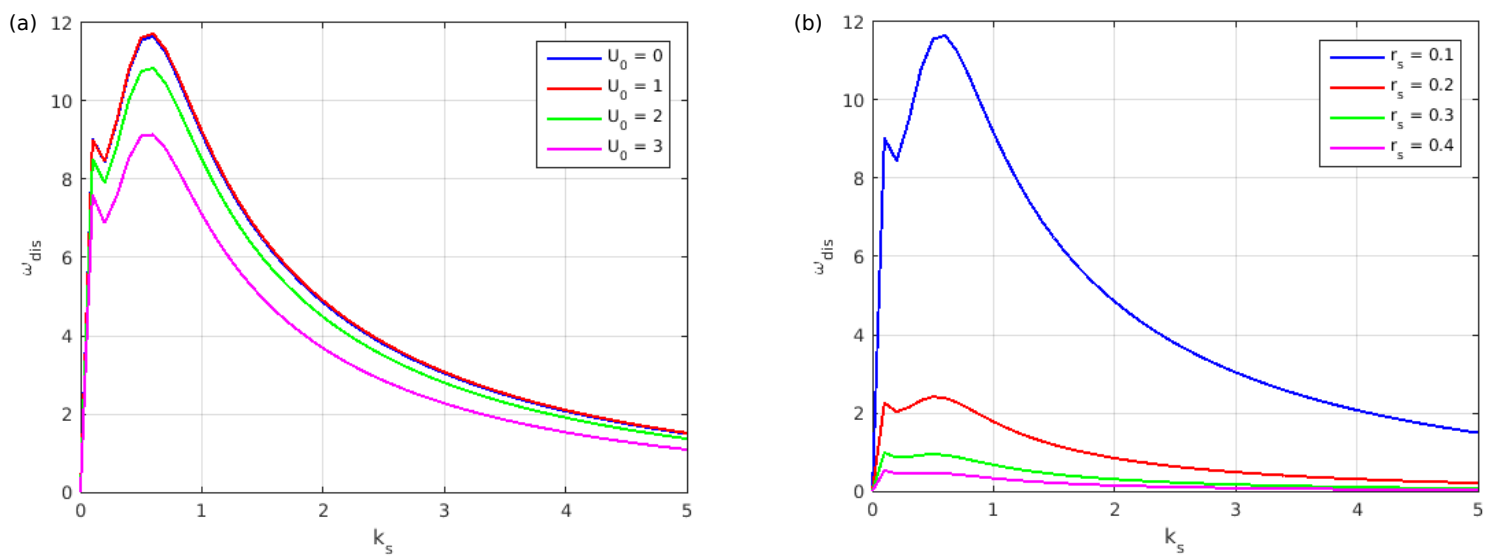

Figura 2.24 - (a) Ritmo de disipación $\omega_{\text {dis }}$ en función de la velocidad axial en el orificio $u_{0_{s t}}$. El resto de los parámetros son: $r_{s_{s t}}=0,1, R_{s}=4$. (b) Ritmo de disipación $\omega_{d i s}$ en función del radio del núcleo de aire en la cámara $r_{s t}$. El resto de los parámetros son: $u_{0_{s t}}=0, R_{s}=4$.

\subsection{Propagación de ondas en la superficie del núcleo de aire en una cámara de sección lentamente va- riable}

En el caso en que la sección de la cámara sea lentamente variable, la región de transición entre la cámara y el orificio no se puede considerar compacta. De una manera similar al estudio de propagación de ondas en aguas de profundidad no uniforme realizado por 
Keller (1958), se ha propuesto una solución asintótica para la propagación de ondas en la cámara de un atomizador cuyo radio varía suavemente. Sea $R_{w}(x)$ el radio de la pared de la cámara, variable con la distancia axial, de forma que la longitud característica de variación de dicho radio es grande frente a la longitud de onda, y $r_{a c}(x)$ el radio del núcleo de aire. El problema a resolver es el problema de frontera libre definido por la ecuación de Laplace (2.8), con las correspondientes condiciones de contorno (2.9) y (2.10), esto es:

$$
\begin{gathered}
\frac{\partial^{2} \varphi}{\partial x^{2}}+\frac{1}{r} \frac{\partial}{\partial r}\left(r \frac{\partial \varphi}{\partial r}\right)=0 \\
\frac{\partial r_{a c}}{\partial t}+\frac{\partial \varphi}{\partial x} \frac{\partial r_{a c}}{\partial x}-\frac{\partial \varphi}{\partial r}=0 \quad \text { en } \quad r=r_{a c} \\
\frac{\partial \varphi}{\partial t}+\frac{1}{2}\left(\frac{\partial \varphi}{\partial x}\right)^{2}+\frac{1}{2}\left(\frac{\partial \varphi}{\partial r}\right)^{2}+\frac{\Gamma^{2}}{8 \pi^{2} r_{a c}^{2}}+\frac{p_{a}}{\rho}=C(t) \quad \text { en } \quad r=r_{a c} \\
\frac{\partial \varphi}{\partial r}-\frac{\mathrm{d} R_{w}}{\mathrm{~d} x} \frac{\partial \varphi}{\partial x}=0 \quad \text { en } \quad r=R_{w}
\end{gathered}
$$

donde $\varphi, \rho, \Gamma$ y $p_{a}$ son el potencial de velocidades y la densidad del líquido, la circulación y la presión del ambiente, respectivamente.

El desarrollo completo de este análisis se incluye en el Apéndice C. Llamando $u_{s}, R_{s} \mathrm{y}$ $r_{s}$ a la velocidad axial del líquido, el radio de la pared de la cámara y el radio del núcleo de aire aguas arriba de la región donde la sección varía, el caudal será $Q=\pi u_{s}\left(R_{s}^{2}-r_{s}^{2}\right)$. Escalando la distancia radial con $R_{s}$, la distancia axial con $\beta R_{s}$, siendo $\beta \gg 1$, el potencial de velocidad con $\Gamma /(2 \pi)$ y el tiempo con $2 \pi R_{s}^{2} / \Gamma$, las ecuaciones (2.139) - (2.142) quedan, en variables adimensionales:

$$
\begin{gathered}
\frac{1}{\beta^{2}} \frac{\partial^{2} \varphi}{\partial x^{2}}+\frac{1}{r} \frac{\partial}{\partial r}\left(r \frac{\partial \varphi}{\partial r}\right)=0 \\
\frac{\partial r_{a c}}{\partial t}-\frac{\partial \varphi}{\partial r}+\frac{1}{\beta^{2}} \frac{\partial \varphi}{\partial x} \frac{\partial r_{a c}}{\partial x}=0 \quad \text { en } r=r_{a c} \\
\frac{\partial \varphi}{\partial t}+\frac{1}{2}\left(\frac{\partial \varphi}{\partial r}\right)^{2}+\frac{1}{2 r_{a c}^{2}}+\frac{1}{2 \beta^{2}}\left(\frac{\partial \varphi}{\partial x}\right)^{2}=\frac{1}{2} u_{s}^{2}+\frac{1}{2 r_{s}^{2}} \quad \text { en } \quad r=r_{a c} \\
\frac{\partial \varphi}{\partial r}-\frac{1}{\beta^{2}} \frac{\mathrm{d} R_{w}}{\mathrm{~d} x} \frac{\partial \varphi}{\partial x}=0 \quad \text { en } \quad r=R_{w}
\end{gathered}
$$

Descomponemos la solución del problema en una componente estacionaria, $\varphi_{s t} \mathrm{y} r_{a c_{s t}}, \mathrm{y}$ otra dependiente del tiempo, $\varphi_{p} \mathrm{y} \eta$, de forma que $\varphi_{p} \ll \varphi_{s t} \mathrm{y} \eta \ll r_{a c_{s t}}$ :

$$
\varphi=\varphi_{s t}(x, r)+\varphi_{p}(x, r, t) \quad \mathrm{y} \quad r_{a c}=r_{a c_{s t}}(x)+\eta(x, t)
$$


Las ecuaciones (2.143) - (2.146) en régimen estacionario quedan:

$$
\begin{gathered}
\frac{1}{\beta^{2}} \frac{\partial^{2} \varphi_{s t}}{\partial x^{2}}+\frac{1}{r} \frac{\partial}{\partial r}\left(r \frac{\partial \varphi_{s t}}{\partial r}\right)=0 \\
-\frac{\partial \varphi_{s t}}{\partial r}+\frac{1}{\beta^{2}} \frac{\partial \varphi_{s t}}{\partial x} \frac{\mathrm{d} r_{a c_{s t}}}{\mathrm{~d} x}=0 \quad \text { en } \quad r=r_{a c_{s t}} \\
\frac{1}{2}\left(\frac{\partial \varphi_{s t}}{\partial r}\right)^{2}+\frac{1}{2 r_{a c_{s t}}^{2}}+\frac{1}{2 \beta^{2}}\left(\frac{\partial \varphi_{s t}}{\partial x}\right)^{2}=\frac{1}{2} u_{s}^{2}+\frac{1}{2 r_{s}^{2}} \quad \text { en } \quad r=r_{a c_{s t}} \\
\frac{\partial \varphi_{s t}}{\partial r}-\frac{1}{\beta^{2}} \frac{\mathrm{d} R_{w}}{\mathrm{~d} x} \frac{\partial \varphi_{s t}}{\partial x}=0 \quad \text { en } \quad r=R_{w}
\end{gathered}
$$

Desarrollando $\varphi_{s t}$ y $r_{a c_{s t}}$ en potencias de $\beta^{-1}$ :

$$
\varphi_{s t}=\beta\left(\varphi_{s t 0}+\frac{1}{\beta^{2}} \varphi_{s t 2}+\ldots\right) \quad ; \quad r_{a c_{s t}}=r_{a c_{s t 0}}+\frac{1}{\beta^{2}} r_{a c_{s t 2}}+\ldots
$$

La solución estacionaria del potencial de velocidades queda:

$$
\varphi_{s t}=\beta f_{0}(x)+\frac{1}{\beta}\left(-\frac{r^{2}}{4} \frac{\mathrm{d}^{2} f_{0}(x)}{\mathrm{d} x^{2}}+f_{2}(x)+g_{2}(x) \ln r\right)+\ldots
$$

Introduciendo (2.153) en (2.149) - (2.151), y tomando los términos de mayor orden, se llega al siguiente sistema de dos ecuaciones para el radio del núcleo de aire, $r_{a c_{s t 0}}$, y para la velocidad axial, $u_{s t 0}=\mathrm{d} f_{0} / \mathrm{d} x$ :

$$
\begin{aligned}
& u_{s t 0}^{2}+\frac{1}{r_{a c_{s t 0}}^{2}}=u_{s}^{2}+\frac{1}{r_{s}^{2}} \\
& u_{s t 0}\left(R_{w}^{2}-r_{a c_{s t 0}}^{2}\right)=\frac{Q}{\pi}
\end{aligned}
$$

Combinando (2.154) y (2.155) se obtiene la siguiente expresión para $r_{a c_{s t 0}}$ :

$$
u_{s}^{2}\left(\frac{1-r_{s}^{2}}{R_{w}^{2}-r_{a c_{s t 0}}^{2}}\right)^{2}+\frac{1}{r_{a c_{s t 0}}^{2}}=u_{s}^{2}+\frac{1}{r_{s}^{2}}
$$

La figura 2.25 muestra la evolución de la velocidad axial $u_{s t 0}$ y del radio del núcleo de aire $r_{a c_{s t 0}}$ con el radio de la pared de la cámara $R_{w}$. Se observa que cuando dicho radio disminuye, aumenta la velocidad axial, lo que da lugar a una disminución de la presión, por lo que también aumenta el radio del núcleo de aire. 

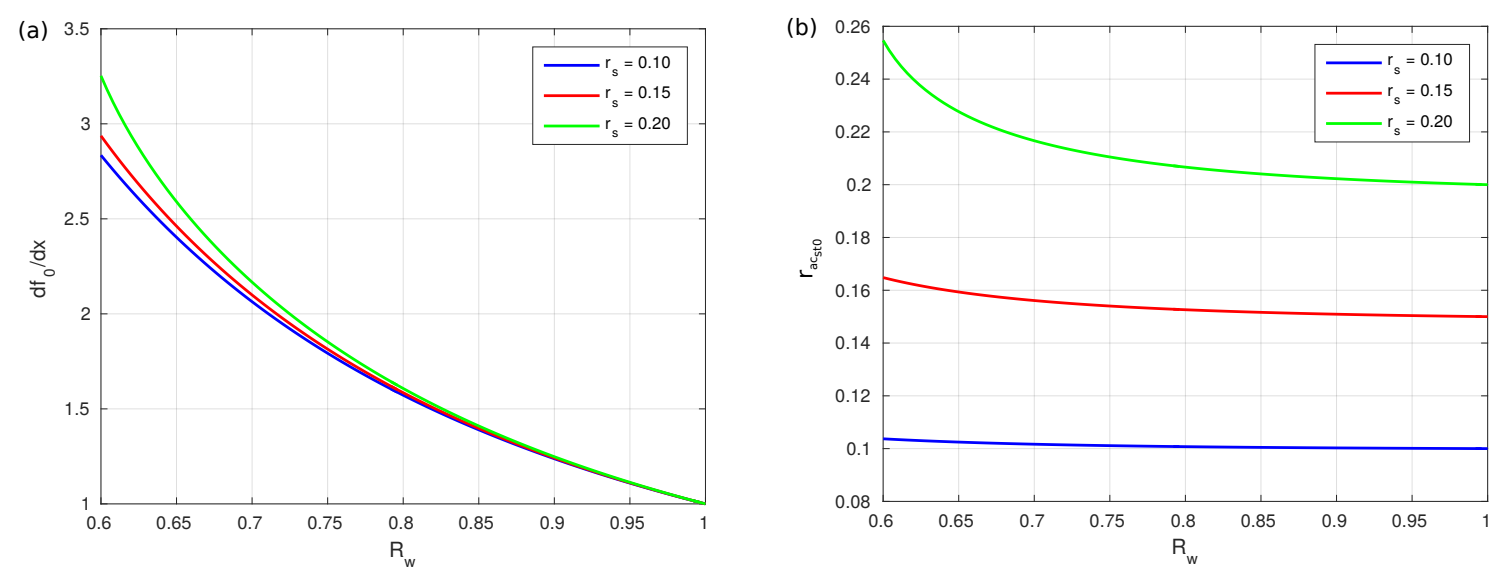

Figura 2.25 - (a) Velocidad axial y (b) radio del núcleo de aire en función del radio de la cámara, para diferentes valores de $r_{s}\left(u_{s}=1\right)$.

Las ecuaciones que gobiernan el problema dependiente del tiempo, linealizadas, son las siguientes:

$$
\begin{gathered}
\frac{1}{\beta^{2}} \frac{\partial^{2} \varphi_{p}}{\partial x^{2}}+\frac{1}{r} \frac{\partial}{\partial r}\left(r \frac{\partial \varphi_{p}}{\partial r}\right)=0 \\
\frac{\partial \eta}{\partial t}-\frac{\partial \varphi_{p}}{\partial r}+\frac{1}{\beta^{2}}\left(\frac{\partial \varphi_{s t}}{\partial x} \frac{\partial \eta}{\partial x}+\frac{\partial \varphi_{p}}{\partial x} \frac{\mathrm{d} r_{a c_{s t}}}{\mathrm{~d} x}\right)=0 \quad \text { en } \quad r=r_{a c_{s t 0}} \\
\frac{\partial \varphi_{p}}{\partial t}+\frac{\partial \varphi_{s t}}{\partial r} \frac{\partial \varphi_{p}}{\partial r}+\frac{1}{\beta^{2}} \frac{\partial \varphi_{s t}}{\partial x} \frac{\partial \varphi_{p}}{\partial x}-\frac{\eta}{r_{a c_{s t}}^{3}}=0 \quad \text { en } \quad r=r_{a c_{s t 0}} \\
\frac{\partial \varphi_{p}}{\partial r}-\frac{1}{\beta^{2}} \frac{\mathrm{d} R_{w}}{\mathrm{~d} x} \frac{\partial \varphi_{p}}{\partial x}=0 \quad \text { en } \quad r=R_{w}
\end{gathered}
$$

Como los coeficientes de las ecuaciones del problema lineal son independientes del tiempo, podemos buscar soluciones de la forma:

$$
\varphi_{p}=\hat{\varphi}_{p}(x, r) e^{i \omega t}+c c \quad \text { y } \quad \eta=\hat{\eta}(x) e^{i \omega t}+c c
$$

donde $c c$ indica complejo conjugado. Se resolverá el problema aplicando el método WKB. Para ello introducimos tres nuevas funciones desconocidas, $A(x, r), D(x)$ y $s(x)$, y expresamos $\hat{\varphi}_{p} \mathrm{y} \hat{\eta}$ en términos de estas funciones mediante las relaciones:

$$
\hat{\varphi}_{p}=A(x, r) e^{i \beta s(x)} \quad \text { y } \quad \hat{\eta}=D(x) e^{i \beta s(x)}
$$

Para valores grandes de $\beta$, las funciones $A(x, r)$ y $D(x)$ tienen unos desarrollos asintóticos de la siguiente forma:

$$
A \sim A_{0}(x, r)+\sum_{n=1}^{\infty} \frac{A_{n}(x, r)}{(i \beta)^{n}} \quad \text { y } \quad D \sim D_{0}(x)+\sum_{n=1}^{\infty} \frac{D_{n}(x)}{(i \beta)^{n}}
$$


2.6. Propagación de ondas en la superficie del núcleo de aire en una cámara de sección lentamente variable

Introduciendo (2.161) - (2.163) en (2.157) - (2.160), y tomando los términos de mayor orden de $\beta$, se obtiene:

$$
A_{0}=a(x) F(x, r)
$$

con

$$
F(x, r)=\frac{K_{0}(k r)}{K_{0}^{\prime}\left(k R_{w}\right)}-\frac{I_{0}(k r)}{I_{0}^{\prime}\left(k R_{w}\right)}
$$

donde $k=|\mathrm{d} s / \mathrm{d} x|$. La relación entre $\omega$ y $k$ es:

$$
\left(\omega+u_{s t 0} k\right)^{2}=-\frac{k}{r_{a c_{s t 0}}^{3}} \frac{K_{0}^{\prime}\left(k r_{a c_{s t 0}}\right) I_{0}^{\prime}\left(k R_{w}\right)-K_{0}^{\prime}\left(k R_{w}\right) I_{0}^{\prime}\left(k r_{a c_{s t 0}}\right)}{K_{0}\left(k r_{a c_{s t 0}}\right) I_{0}^{\prime}\left(k R_{w}\right)-K_{0}^{\prime}\left(k R_{w}\right) I_{0}\left(k r_{a c_{s t 0}}\right)}
$$

La expresión (2.166) coincide con la relación de dispersión para una cámara de radio constante igual al valor local de $R_{w}$, dada por (2.117).

En la figura 2.26 se muestra la variación del número de onda $k$ con el radio de la cámara $R_{w}$, dada por la expresión (2.166), para varios valores de $r_{s} \mathrm{y}$ de $\omega$. Se observa que $k$ disminuye cuando $R_{w}$ aumenta, y aumenta cuando lo hacen $r_{s} \mathrm{y} \omega$.
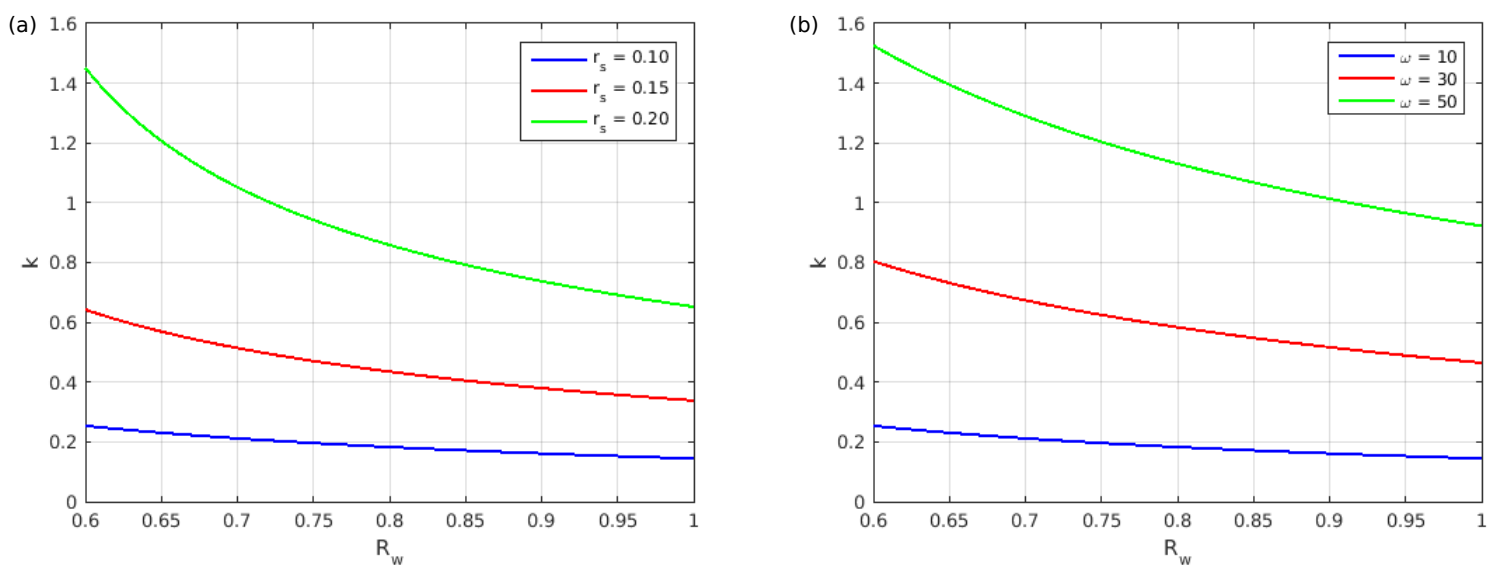

Figura 2.26 - Variación del número de onda $k$ en función del radio de la pared de la cámara $R_{w}$, para varios valores de (a) $r_{s}\left(\omega=10, u_{s}=0,5\right)$ y de (b) $\omega\left(r_{s}=0,1\right.$, $\left.u_{s}=0,5\right)$.

Tomando ahora los términos de orden $\beta^{-1}$ se llega a la siguiente expresión para la variación de la amplitud de las perturbaciones:

$$
\frac{\partial}{\partial x}\left[a^{2}\left(k \int_{r_{a c_{s t 0}}}^{R_{w}} F^{2} r \mathrm{~d} r-F^{2}\left(x, r_{a c_{s t 0}}\right) r_{a c_{s t 0}}^{4} u_{s t 0}\left(\omega+u_{s t 0} k\right)\right)\right]=0
$$


Esta expresión es equivalente a (ver Apéndice C):

$$
\frac{\partial}{\partial x}\left(\frac{\left(u_{s t 0}+c_{g}\right) E}{\omega+u_{s t 0} k}\right)=0
$$

donde $c_{g}$ es la velocidad de grupo y $\left(u_{s t 0}+c_{g}\right) E$ es el flujo de energía de las ondas, que se define, de acuerdo con Lighthill (1978), de la siguiente manera:

$$
\left(u_{s t 0}+c_{g}\right) E=\int_{r_{a c_{s t}+\eta}}^{R_{w}} \overline{p_{p} \frac{\partial \varphi}{\partial x}} 2 \pi r \mathrm{~d} r
$$

donde la línea sobre las variables indica valor promedio, y $p_{p}$ es la perturbación en la presión, que viene dada por:

$$
p_{p}=-\frac{\partial \varphi_{p}}{\partial t}-\frac{\partial \varphi_{s t}}{\partial r} \frac{\partial \varphi_{p}}{\partial r}-\frac{1}{\beta^{2}} \frac{\partial \varphi_{s t}}{\partial x} \frac{\partial \varphi_{p}}{\partial x}
$$

Esto es, el flujo de la acción de ondas, definido como $\left(u_{s t 0}+c_{g}\right) E /\left(\omega+u_{s t 0} k\right)$, se conserva. En otras palabras, la relación entre el flujo de energía de las ondas y su frecuencia permanece constante a lo largo de la cámara. En una determinada sección de la cámara, la energía de las ondas aumentará si se aumenta la frecuencia. Asímismo, para una frecuencia fija, la energía de las ondas aumentará al disminuir la sección de la cámara.

Llamando $a_{s}$ y $k_{s}$ a los valores que toman, respectivamente, la amplitud y el número de onda en la sección donde $R_{w}=1$ :

$$
\frac{a}{a_{s}}=\frac{k_{s} \int_{r_{s}}^{1} F^{2} r \mathrm{~d} r-F^{2}\left(x, r_{s}\right) r_{s}^{4} u_{s}\left(\omega+u_{s} k_{s}\right)}{k \int_{r_{a c_{s t 0}}}^{R_{w}} F^{2} r \mathrm{~d} r-F^{2}\left(x, r_{a c_{s t 0}}\right) r_{a c_{s t 0}}^{4} u_{s t 0}\left(\omega+u_{s t 0} k\right)}
$$

donde $F(x, r)$ y $k(x)$ vienen dadas por (2.165) y (2.166), respectivamente.
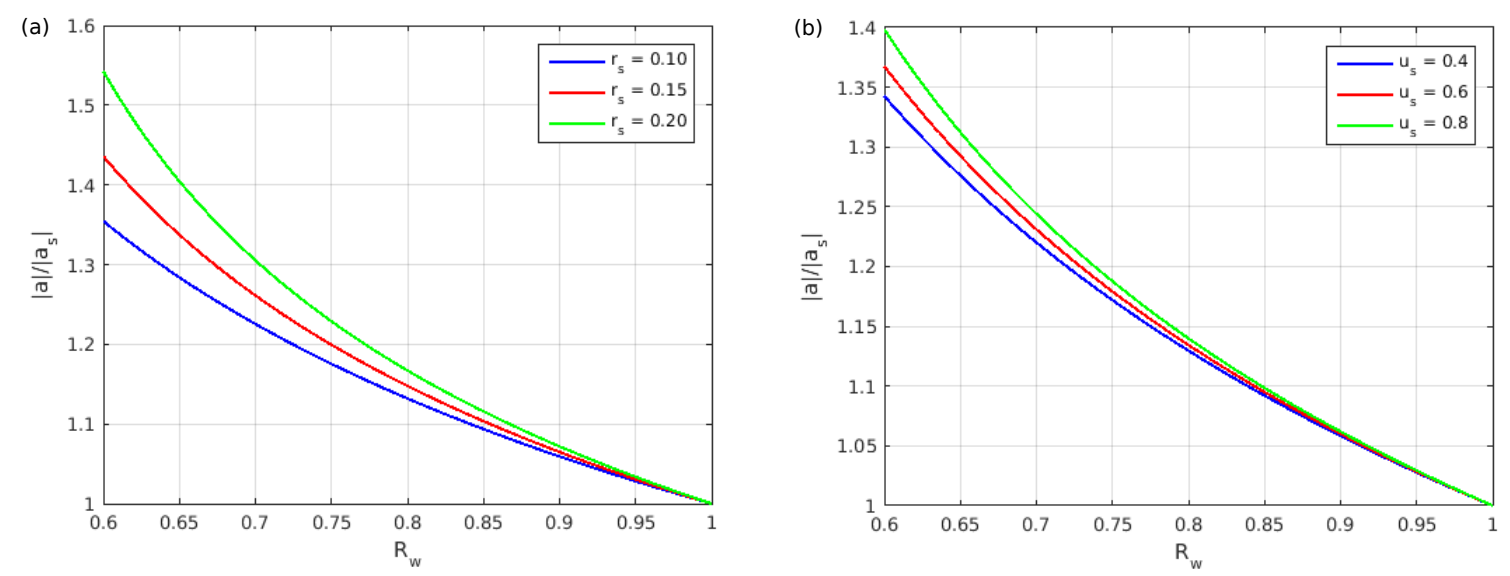

Figura 2.27 - Variación de la amplitud de las perturbaciones en función del radio de la pared de la cámara $R_{w}$, para varios valores de $(\mathrm{a}) r_{s}(\omega=10)$ y de $(\mathrm{b}) \omega\left(r_{s}=0,1\right)$. 
2.6. Propagación de ondas en la superficie del núcleo de aire en una cámara de sección lentamente variable

En la figura 2.27 se muestra la variación de la amplitud en función del radio de la cámara $R_{w}$, para varios valores de $r_{s} \mathrm{y}$ de $\omega$. Se observa que, efectivamente, el valor de la amplitud aumenta cuando lo hace la frecuencia en una determinada sección de la cámara, y también aumenta cuando disminuye la sección de la misma para un valor fijo de la frecuencia. 


\section{Capítulo 3}

\section{Efecto de la viscosidad}

En el capítulo anterior se han estudiado modelos irrotacionales para el flujo en el atomizador. Dichos modelos no tienen en cuenta la viscosidad. Si se consideran los efectos de la misma, se formarán capas límite en las paredes internas del atomizador. El gradiente radial de presión que acompaña al giro del líquido en el interior del atomizador también es impuesto en estas capas límite. Sin embargo, la velocidad de giro cae a cero en las paredes, por lo que la aceleración centrípeta se reduce en las capas límite, de manera que no puede equilibrar el gradiente de presión radial en las paredes que no son paralelas al eje del atomizador. Debido a ello, el gradiente de presión empuja el líquido hacia el eje del atomizador. Este efecto se conoce como bombeo de Ekman. En la capa límite de la pared convergente de la cámara el líquido se mueve hacia el orificio de salida, y en la capa límite de la pared opuesta al orificio de salida el líquido se mueve hacia el núcleo de aire y, cuando lo alcanza, gira y fluye rodeándolo hacia el orificio de salida. Las capas límite que aparecen de esta forma a ambos lados de la lámina anular que emerge del orificio pueden cubrir una parte importante del espesor de la lámina, como ya indicó Taylor (1948), lo que limita la validez de los modelos irrotacionales. Las capas límite han sido analizadas mediante métodos analíticos (Binnie \& Harris 1950; Taylor 1950; Cooke 1952), experimentales (Binnie, Hookings \& Kamel 1957) y numéricos (Burggraf, Stewartson \& Belcher 1971; Bloor \& Ingham 1977; Dumouchel et al. 1992).

Los análisis realizados por Binnie \& Harris (1950), Taylor (1950) y Cooke (1952) ofrecen bastante información sobre el crecimiento de la capa límite que se forma sobre la pared de la cámara, confirmando la posibilidad de que gran parte del líquido abandona el inyector a través de la capa límite. Por otro lado, los experimentos realizados por Binnie, Hookings \& Kamel (1957) sobre flujos giratorios en toberas mostraron la presencia de valores altos de la componente axial de la velocidad del líquido en las proximidades del 
núcleo de aire, lo que indica la existencia de la capa límite alrededor del mismo. Los estudios numéricos llevados a cabo por Burggraf, Stewartson \& Belcher (1971) y Bloor \& Ingham (1977) acerca del flujo giratorio sobre un disco plano dieron una idea de la estructura de la capa límite en la base de la cámara. Estos estudios fueron adaptados por Dumouchel et al. (1992) al caso del flujo giratorio en una tobera cónica para analizar la influencia de ciertos parámetros geométricos y de operación en el crecimiento de la capa límite en las paredes de la cámara. Una descripción más detallada de estos estudios se incluye en la Sección 1.3.

Las características del flujo antes descritas sugieren analizar el problema mediante un modelo de capa límite (Higuera \& Pereña 2014). Sin embargo, en la cámara del atomizador se genera recirculación para valores bajos y moderados de la constante del atomizador, según se ha observado mediante experimentos (Binnie et al. 1957; Wang et al. 1999; Donjat et al. 2003) y simulaciones numéricas (Jeng et al. 1998; Yule \& Chinn 2000; NouriBorujerdi \& Kebriaee 2012). Esta recirculación es difícil de calcular mediante un modelo de capa límite. El tamaño de la región de recirculación y la dirección de la circulación del líquido dependen de las condiciones de entrada. Esta dirección es contraria en simulaciones realizadas inyectando el líquido con una componente radial de velocidad (Jeng et al. 1998; Yule \& Chinn 2000; Xue et al. 2004) frente a simulaciones en las que el líquido se inyecta con una componente axial de velocidad (Nouri-Borujerdi \& Kebriaee 2012). Sin embargo estas diferencias no dan lugar a un efecto importante en las características del atomizador, probablemente porque las componentes de la velocidad del flujo recirculatorio en una sección axial de la cámara son pequeñas comparadas con la velocidad azimutal en otras regiones de la cámara y con la velocidad en el orificio de salida. Estos resultados sugieren que un modelo de capa límite puede capturar muchas características importantes del flujo en la cámara del atomizador, y todo ello a una fracción del coste que supondría una simulación numérica empleando las ecuaciones de Navier-Stokes completas.

Otra limitación de la aproximación de capa límite se debe a la presencia de inestabilidades centrífugas en el flujo giratorio en la capa límite sobre la pared curva de la cámara (Rayleigh 1916). Es aplicable el razonamiento clásico para partículas fluidas que se mueven siguiendo trayectorias circulares. Sean $r$ y $w$ el radio y la velocidad azimutal de una partícula fluida. Es necesaria una fuerza centrípeta, de magnitud $\rho w^{2} / r$ por unidad de volumen, para que el movimiento sea estacionario, y esta fuerza es aportada por el gradiente de presión $-\partial p / \partial r$. Consideremos ahora una partícula fluida con velocidad azimutal $w_{1}$ a un radio $r_{1}$, y supongamos que dicha partícula se desplaza a un radio $r_{2}$ que es mayor que $r_{1}$. Si el momento angular de la partícula permanece invariable, su nueva velocidad será 
$r_{1} w_{1} / r_{2}$. Para que la partícula estuviera en equilibrio en su nuevo radio, se requeriría una fuerza centrípeta de valor $\rho r_{1}^{2} w_{1}^{2} / r_{2}^{3}$. Sin embargo, el gradiente de presión a un radio $r_{2}$ ejerce una fuerza hacia el interior de magnitud $\rho w_{2}^{2} / r_{2}$, donde $w_{2}$ es la velocidad a un radio $r_{2}$ y esta fuerza no es igual a la fuerza centrípeta requerida por la partícula fluida recién llegada. De hecho, $\rho w_{2}^{2} / r_{2}<\rho r_{1}^{2} w_{1}^{2} / r_{2}^{3}$, pues $w_{2}<r_{1} w_{1} / r_{2}$, por la presencia de la capa límite. Debido a ello, la partícula tenderá a alejarse de su posición, por lo que el flujo es inestable. Este problema fue estudiado teóricamente por Görtler (1940), quien mostró que esta inestabilidad genera torbellinos cuyos ejes son paralelos a la pared, como se muestra en la figura 3.1. La confirmación experimental de esta inestabilidad fue encontrada por Gregory \& Walker (1950).

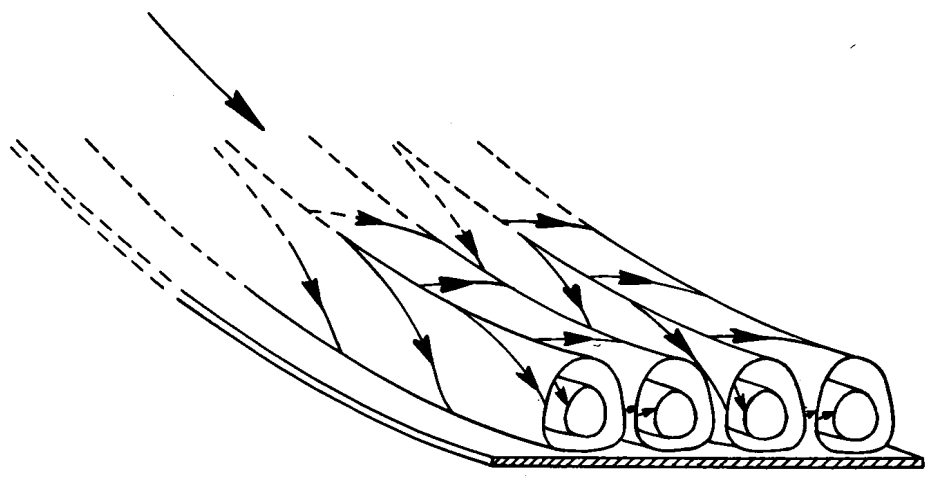

Figura 3.1 - Torbellinos generados en el flujo a lo largo de una pared cóncava. Görtler (1940).

Como han notado Yule \& Chinn (2000), esta inestabilidad se origina en la región cilíndrica que precede a la convergencia hacia el orificio de salida, pues aquí no se produce bombeo de Ekman y la velocidad axial es muy pequeña para barrer las perturbaciones. Debido a ello, para prevenir esta inestabilidad, la superficie cilíndrica ha sido reemplazada en el análisis de este capítulo por una superficie ligeramente cónica y convergente, situada aguas arriba de la convergencia principal.

\subsection{Formulación de un modelo cuasi-cilíndrico}

La figura 3.2 muestra un esquema de la cámara axilsimétrica de un atomizador de presión con giro, de radio $R_{w}(x)$, donde $x$ es la distancia axial a lo largo de la cámara. El líquido es inyectado en la cámara a un caudal constante $Q$ a través de canales tangenciales localizados en la periferia cerca de la base de la cámara opuesta al orificio de salida (no 
mostrada en la figura), lo cual induce una alta velocidad azimutal, y se descarga a través de un orificio central de radio $R_{0}$.

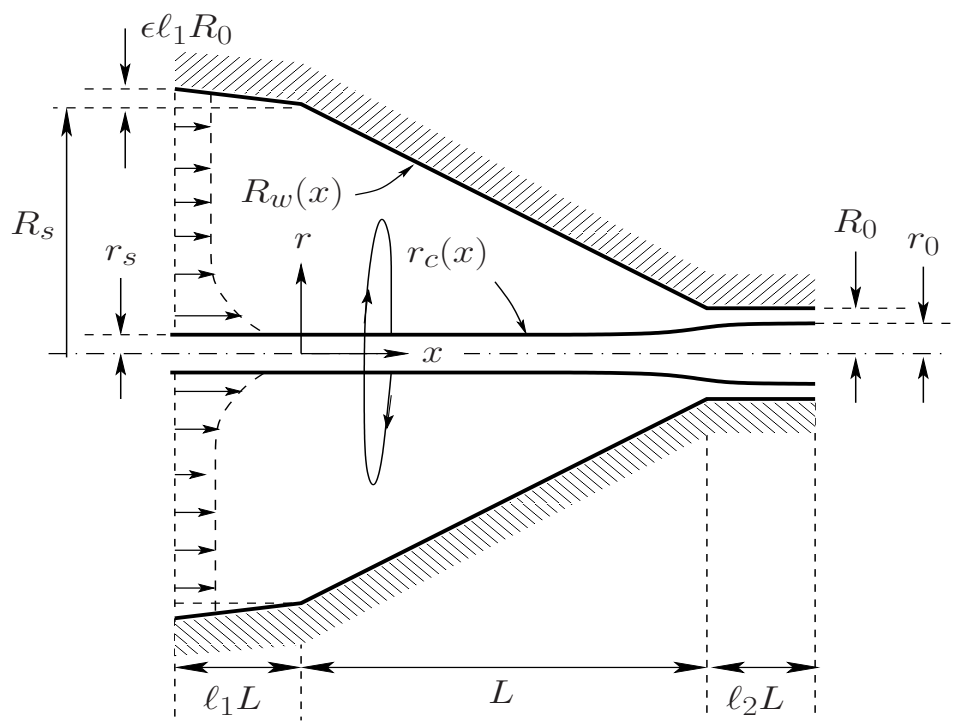

Figura 3.2 - Esquema de la cámara del atomizador

Para el análisis se empleará una aproximación cuasi-cilíndrica para flujos axisimétricos giratorios, que es válida cuando el flujo es esbelto $\left(L \gg R_{s}\right.$ y $u \gg v$, ver figura 3.2) y la mayor contribución a la componente axial de la aceleración se debe al movimiento azimutal del líquido $\left(w \gg u R_{s} / L\right)$. En estas condiciones, las derivadas axiales en los términos viscosos de las ecuaciones de cantidad de movimiento se pueden despreciar, y la ecuación de cantidad de movimiento en dirección radial se reduce al equilibrio entre la fuerza de presión y la aceleración radial debida al giro. En coordenadas cilíndricas, las ecuaciones que gobiernan el problema son las siguientes (Batchelor 1967):

$$
\begin{gathered}
\frac{\partial u}{\partial x}+\frac{1}{r} \frac{\partial}{\partial r}(r v)=0 \\
u \frac{\partial u}{\partial x}+v \frac{\partial u}{\partial r}=-\frac{1}{\rho} \frac{\partial p}{\partial x}+\nu \frac{1}{r} \frac{\partial}{\partial r}\left(r \frac{\partial u}{\partial r}\right) \\
\frac{1}{\rho} \frac{\partial p}{\partial r}=\frac{w^{2}}{r} \\
u \frac{\partial}{\partial x}(r w)+v \frac{\partial}{\partial r}(r w)=\nu r \frac{\partial}{\partial r}\left(\frac{1}{r} \frac{\partial}{\partial r}(r w)\right)
\end{gathered}
$$

donde $\nu$ representa la viscosidad cinemática del líquido. Escalamos la distancia axial con $L$, la distancia radial con $R_{0}$, las velocidades axial y azimutal con $\Gamma /\left(2 \pi R_{0}\right)$, la 
velocidad radial con $\Gamma /(2 \pi L)$ y la presión con $\rho \Gamma^{2} /\left(4 \pi^{2} R_{0}^{2}\right)$, donde $L$ es la longitud del tramo convergente (ver figura 3.2). Definiendo el número de Reynolds efectivo como $R e=$ $(\Gamma /(2 \pi \nu))\left(R_{0} / L\right)$, las ecuaciones adimensionales son las siguientes:

$$
\begin{gathered}
\frac{\partial u}{\partial x}+\frac{1}{r} \frac{\partial}{\partial r}(r v)=0 \\
u \frac{\partial u}{\partial x}+v \frac{\partial u}{\partial r}=-\frac{\partial p}{\partial x}+\frac{1}{R e} \frac{1}{r} \frac{\partial}{\partial r}\left(r \frac{\partial u}{\partial r}\right) \\
\frac{\partial p}{\partial r}=\frac{w^{2}}{r} \\
u \frac{\partial}{\partial x}(r w)+v \frac{\partial}{\partial r}(r w)=\frac{1}{R e} r \frac{\partial}{\partial r}\left(\frac{1}{r} \frac{\partial}{\partial r}(r w)\right)
\end{gathered}
$$

Se han despreciado las variaciones de presión en el aire y los esfuerzos viscosos del aire sobre la superficie del líquido, así como la tensión superficial. Las condiciones de contorno en la pared de la cámara, $r=R_{w}(x)$, y en la superficie de separación entre el líquido y el aire, $r=r_{a c}(x)$, la cual se ha de hallar como parte de la solución, son:

$$
\begin{gathered}
r=R_{w}(x): \quad u=v=w=0 \\
r=r_{a c}(x): \quad v-u \frac{\mathrm{d} r_{a c}}{\mathrm{~d} x}=p=\frac{\partial u}{\partial r}=\frac{\partial}{\partial r}\left(\frac{w}{r}\right)=0
\end{gathered}
$$

La región de la cámara donde se aplican las ecuaciones (3.5)-(3.8) consiste en una región suavemente convergente de longitud adimensional $\ell_{1}$ (escalada con $L$ ) que finaliza en un radio adimensional $R_{s}$ (escalado con $R_{0}$ ), seguida de una convergencia cónica mucho más pronunciada de longitud y radio final adimensionales de valor unidad, y de una región cilíndrica de longitud adimensional $\ell_{2}$ (escalada con $L$ ) y radio adimensional unidad. De esta manera, situando el origen de la coordenada espacial axial al comienzo de la convergencia cónica fuerte, la pared de la cámara queda definida de la siguiente manera:

$$
R_{w}(x)=\left\{\begin{array}{lll}
R_{s}-\epsilon x & \text { si } & -\ell_{1}<x<0 \\
R_{s}-\left(R_{s}-1\right) x & \text { si } & 0<x<1 \\
1 & \text { si } & 1<x<1+\ell_{2}
\end{array}\right.
$$

El parámetro $\epsilon$ indica la suave contración de la cámara. El valor $\epsilon=0$ correspondería con una cámara cilíndrica antes de la contracción cónica. En los cálculos posteriores se emplearán valores de $\epsilon=0,1-0,2$ para evitar la inestabilidad centrífuga mencionada antes.

Las condiciones de contorno a la entrada y a la salida requieren alguna discusión adicional. 


\subsubsection{Condición de contorno a la entrada}

Debido a que la aproximación cuasi-cilíndrica no puede describir el flujo alrededor de los canales de entrada y en la capa límite en la pared de la cámara opuesta al orificio de salida, se han impuesto unas condiciones en $x=-\ell_{1}$ que aproximan el flujo real. Estas condiciones son:

$$
x=-\ell_{1}: \quad u=u_{s 0}+u_{j e t}(r), \quad w=\frac{1}{r}-w_{j e t}(r)
$$

En la expresión anterior, $u_{s 0}$ representa una velocidad axial uniforme, y $u_{j e t}(r)$ y $w_{j e t}(r)$ son perfiles similares a un chorro que dan cuenta del exceso de velocidad axial y del defecto de velocidad azimutal alrededor del núcleo de aire debidos a la capa límite que se desarrolla sobre la base de la cámara, que converge radialmente sobre esta base y se reorienta al alcanzar el núcleo de aire. Dichos perfiles han de obtenerse de un análisis de dicha capa límite, que ha sido llevado a cabo a varios niveles de detalle considerando que la base de la cámara es un disco plano y que el líquido se inyecta simétricamente. Sin embargo, el flujo alrededor de la base de la cámara puede ser muy complejo para otras configuraciones de la pared y de los canales de entrada. Esto supone una limitación adicional de la aproximación cuasi-cilíndrica. En el análisis que sigue se empleará la siguiente aproximación para los perfiles de velocidad:

$$
u_{j e t}(r)=u_{c} \exp \left[-\frac{\left(r-r_{a c}\left(-\ell_{1}\right)\right)^{2}}{\delta^{2}}\right], \quad w_{j e t}(r)=\frac{1}{r} \exp \left[-\frac{\left(r-r_{a c}\left(-\ell_{1}\right)\right)^{2}}{\delta^{2}}\right]
$$

Las constantes de la expresión anterior cumplen $u_{c}=O(1)$ y $\delta=O\left(R e^{-1 / 2}\right)$. El caudal adimensional $Q$ (escalado con $\Gamma R_{0}$ ) es conocido, y cumple:

$$
Q=\int_{r_{a c}\left(-\ell_{1}\right)}^{R_{w}\left(-\ell_{1}\right)} 2 \pi r\left(u_{s 0}+u_{j e t}(r)\right) \mathrm{d} r
$$

De la anterior expresión se puede obtener la parte uniforme de la velocidad de entrada:

$$
u_{s 0}=\frac{Q-\int_{r_{a c}\left(-\ell_{1}\right)}^{R_{w}\left(-\ell_{1}\right)} 2 \pi r u_{j e t}(r) \mathrm{d} r}{\pi\left[R_{w}\left(-\ell_{1}\right)^{2}-r_{a c}\left(-\ell_{1}\right)^{2}\right]}
$$

\subsubsection{Condición de contorno a la salida}

Se necesita una condición de contorno al final del orificio $\left(x=1+\ell_{2}\right)$ porque el modelo cuasi-cilíndrico no es parabólico, a pesar de la aproximación de capa límite. Esto está relacionado con el hecho de que el gradiente de presión en la ecuación (3.6) no es dado sino que forma parte de la solución, lo que hace posible la propagación de las ondas aguas 
arriba. El modelo de capa límite falla en una región próxima al final del orificio de salida debido a que aquí el radio de curvatura de las líneas de corriente es del orden del espesor de la capa. La condición de contorno buscada expresa el comportamiento de la solución de (3.5)-(3.8) al aproximarse a esta región. Es una extensión directa de la condición de flujo máximo empleada en los modelos irrotacionales. Esta condición establece que, aproximándose al final del orificio de salida, el flujo se debe acelerar tanto como pueda, en anticipación a la mayor aceleración que experimenta más lejos aguas abajo, donde el movimiento radial del líquido no está restringido por las paredes de la cámara. Bajo la acción del gradiente de presión antes mencionado, los efectos viscosos son confinados a una subcapa cuyo espesor tiende a cero cuando $x \rightarrow 1+\ell_{2}$, mientras que la solución en el resto de la lámina líquida es esencialmente no viscosa, pero con vorticidad. Llamando $r_{0}<1$ al valor límite del radio del núcleo de aire al final del orificio (pero donde (3.5)(3.8) son aún aplicables), y $u^{*}(r), w^{*}(r)$ y $p^{*}(r)$ a las distribuciones límite de velocidad axial, velocidad azimutal y presión, y teniendo en cuenta que cerca del final del orificio las distribuciones de velocidad y presión difieren poco de dichas distribuciones límite, los perfiles fuera de la subcapa viscosa pueden expresarse como desarrollos en potencias de la distancia al final del orificio de la siguiente manera:

$$
\begin{gathered}
u=u^{*}(r)+\left(1+\ell_{2}-x\right)^{\lambda} U(r)+\ldots \\
v=\left(1+\ell_{2}-x\right)^{\lambda-1} V(r)+\ldots \\
w=w^{*}(r)+\left(1+\ell_{2}-x\right)^{\lambda} W(r)+\ldots \\
p=p^{*}(r)+\left(1+\ell_{2}-x\right)^{\lambda} P(r)+\ldots \\
r_{c}=r_{0}^{*}+\left(1+\ell_{2}-x\right)^{\lambda} R+\ldots
\end{gathered}
$$

donde $0<\lambda<1$ ha de ser determinado. Sustituyendo (3.15) en (3.5)-(3.8):

$$
-\lambda U+\frac{(r V)^{\prime}}{r}=0
$$

$$
\begin{aligned}
& \lambda u^{*} U-u^{*^{\prime}} V+\lambda P+\left(1+\ell_{2}-x\right)^{\lambda}\left(\lambda U^{2}-U^{\prime} V\right) \\
&+\left(1+\ell_{2}-x\right)^{1-\lambda} \frac{1}{R e} \frac{\left(r u^{*^{\prime}}\right)^{\prime}}{r}+\left(1+\ell_{2}-x\right) \frac{1}{R e} \frac{\left(r U^{\prime}\right)^{\prime}}{r}=0 \\
& p^{*^{\prime}}-\frac{w^{* 2}}{r}+\left(1+\ell_{2}-x\right)^{\lambda}\left(P^{\prime}-\frac{2 w^{*} W}{r}\right)-\left(1+\ell_{2}-x\right)^{2 \lambda} \frac{W^{2}}{r}=0
\end{aligned}
$$

$$
\begin{aligned}
\lambda r u^{*} W-V & \left(r w^{*}\right)^{\prime}-\left(1+\ell_{2}-x\right)^{\lambda}\left(-\lambda r U W+V(r W)^{\prime}\right) \\
& +\left(1+\ell_{2}-x\right)^{1-\lambda} \frac{1}{R e} r\left(\frac{\left(r w^{*}\right)^{\prime}}{r}\right)^{\prime}+\left(1+\ell_{2}-x\right) \frac{1}{R e} r\left(\frac{(r W)^{\prime}}{r}\right)^{\prime}=0
\end{aligned}
$$


Tomando en las anteriores expresiones los principales términos de los desarrollos:

$$
\begin{gathered}
-\lambda U+\frac{(r V)^{\prime}}{r}=0 \\
\lambda u^{*} U-u^{*^{\prime}} V+\lambda P=0 \\
P^{\prime}-\frac{2 w^{*} W}{r}=0 \\
\lambda r u^{*} W-V\left(r w^{*}\right)^{\prime}=0
\end{gathered}
$$

Derivando en (3.21):

$$
\lambda\left(u^{*} U\right)^{\prime}-\left(u^{*^{\prime}} V\right)^{\prime}+\lambda P^{\prime}=0
$$

Despejando $U, P^{\prime}$ y $W$ de (3.20), (3.22) y (3.23) respectivamente, y sustituyendo en (3.24), se llega a la siguiente expresión para $V$ :

$$
\left[\frac{u^{* 2}}{r}\left(r \frac{V}{u^{*}}\right)^{\prime}\right]^{\prime}+\frac{\left[\left(r w^{*}\right)^{2}\right]^{\prime}}{r^{3}} \frac{V}{u^{*}}=0
$$

Procediendo de la misma forma para las condiciones de contorno (3.10) se tiene:

$$
\frac{V}{u^{*}}=\lambda R \quad \text { y } \quad\left(r \frac{V}{u^{*}}\right)^{\prime}=\lambda \frac{w^{* 2}}{u^{* 2}} R \quad \text { en } \quad r=r_{0}
$$

La velocidad límite ha de ser de la forma $u^{*} \approx \alpha(1-r)^{\sigma}$ y $w^{*} \approx \beta(1-r)^{\tau}$ para ciertos valores $(\sigma, \tau)<1$ cuando $(1-r) \ll 1$, debido al esfuerzo de cortadura infinito que se requiere para hacer frente al gradiente de presión infinito. Un análisis local de la ecuación diferencial (3.26) cuando $(1-r) \ll 1$ muestra que tiene dos soluciones linealmente independientes de la forma $V \sim(1-r)^{\sigma}$ y $V \sim(1-r)^{1-\sigma}$. De la ecuación de continuidad (3.20) se deduce que:

$$
U \sim \frac{1}{(1-r)^{1-\sigma}} \quad \text { y } \quad U \sim \frac{1}{(1-r)^{\sigma}} \quad \text { para } \quad(1-r) \ll 1
$$

El espesor característico $\delta_{v}$ de la subcapa viscosa y una primera relación entre las constantes $\sigma$ y $\lambda$ pueden obtenerse del balance del orden de magnitud de las fuerzas de inercia, de presión y viscosas en la ecuación de cantidad de movimiento axial (3.6):

$$
\frac{u_{v}^{2}}{1+\ell_{2}-x} \sim \frac{\Delta p}{1+\ell_{2}-x} \sim \frac{u_{v}}{R e \delta_{v}^{2}}
$$

donde la velocidad característica $u_{v}$ ha de satisfacer $u_{v} \sim \delta_{v}^{\sigma}$ para poder acoplar la solución con $u^{*}(r)$, y $\Delta p=\left(1+\ell_{2}-x\right)^{\lambda} P(1)$. Estas dos condiciones dan $\delta_{v} \sim\left[\left(1+\ell_{2}-x\right) / R e\right]^{1 /(\sigma+2)}$ y $\lambda=2 \sigma /(\sigma+2)$. 
Introducimos la nueva variable $\eta$, que definimos como:

$$
\eta=\frac{1-r}{\left(\frac{1+\ell_{2}-x}{R e}\right)^{1 /(\sigma+2)}}
$$

y la función de corriente $\psi$, tal que $u=\partial \psi / \partial r$ y $v=-\partial \psi / \partial x$. Teniendo en cuenta que $u_{v} \sim\left[\left(1+\ell_{2}-x\right) / R e\right]^{\sigma /(\sigma+2)}$, buscamos la función de corriente de la siguiente manera:

$$
\psi=-\left(\frac{1+\ell_{2}-x}{R e}\right)^{(\sigma+1) /(\sigma+2)} g(\eta)
$$

Expresando la ecuación de cantidad de movimiento en dirección axial (3.6) en términos de la función de corriente:

$$
\frac{\partial \psi}{\partial r} \frac{\partial^{2} \psi}{\partial x \partial r}-\frac{\partial \psi}{\partial x} \frac{\partial^{2} \psi}{\partial r^{2}}=-\frac{\partial p}{\partial x}+\frac{1}{R e} \frac{1}{r} \frac{\partial}{\partial r}\left(r \frac{\partial^{2} \psi}{\partial r^{2}}\right)
$$

Sustituyendo $\partial p / \partial x=-(2 \sigma / \sigma+2)\left(1+\ell_{2}-x\right)^{(\sigma-2) /(\sigma+2)} P(1)$ y las derivadas de la función de corriente se llega a una ecuación diferencial para $g(\eta)$ :

$$
(\sigma+2) g^{\prime \prime \prime}+\sigma g^{\prime 2}-(\sigma+1) g g^{\prime \prime}+2 \sigma R e^{2 \sigma /(\sigma+2)} P(1)=0
$$

con las condiciones de contorno

$$
g=g^{\prime}=0 \quad \text { en } \quad \eta=0
$$

y la condición de acoplamiento

$$
g^{\prime} \sim \alpha \eta^{\sigma} \quad \text { en } \quad \eta \rightarrow \infty
$$

Para resolver este problema empezaremos determinando la forma de la función $g$ para valores grandes de $\eta$. De la condición de acoplamiento se deduce que el desarrollo de $g$ será de la forma:

$$
g \simeq \frac{\alpha}{\sigma+1} \eta^{\sigma+1}+\tilde{g} \quad \text { para } \quad \eta \rightarrow \infty
$$

donde $\tilde{g} \ll O\left(\eta^{\sigma+1}\right)$. Sustituyendo este desarrollo en la ecuación (3.31) se obtiene, en primera aproximación:

$$
(\sigma+2) \tilde{g}^{\prime \prime \prime}+2 \alpha \sigma \eta^{\sigma} \tilde{g}^{\prime}-\alpha(\sigma+1) \sigma \eta^{\sigma-1} \tilde{g}-\alpha \eta^{\sigma+1} \tilde{g}^{\prime \prime}+2 \sigma R e^{2 \sigma /(\sigma+2)} P(1)=0
$$

Para determinar las posibles soluciones de esta ecuación lineal de tercer orden, probaremos en primer lugar soluciones del tipo $\tilde{g} \sim \beta \eta^{\mu}$, que conducen a:

$$
\begin{aligned}
& \beta(\sigma+2) \mu(\mu-1)(\mu-2) \eta^{\mu-3}+2 \alpha \beta \sigma \mu \eta^{\sigma+\mu-1} \\
& \quad-\alpha \beta(\sigma+1) \sigma \eta^{\sigma+\mu-1}-\alpha \beta \mu(\mu-1) \eta^{\sigma+\mu-1}+2 \sigma R e^{2 \sigma /(\sigma+2)} P(1)=0
\end{aligned}
$$


donde el primer término puede despreciarse para cualquier $\sigma>-2$. Una solución particular que dé cuenta del término forzante se obtiene para $\mu=1-\sigma \mathrm{y}$ :

$$
2 \alpha \beta \sigma(1-2 \sigma)+2 \sigma R e^{2 \sigma /(\sigma+2)} P(1)=0
$$

Dos soluciones linealmente independientes de la ecuación homogénea se obtienen imponiendo que $2 \sigma \mu-\sigma(\sigma+1)-\mu(\mu-1)=0$, que tiene las soluciones $\mu=\sigma$ y $\mu=\sigma+1$. La tercera solución de la ecuación homogénea es de la forma $\tilde{g} \sim b \exp (s)$, donde $s$ es un desarrollo en potencias de $\eta$. Sustituyendo en la ecuación (3.31) y eliminando los términos de menor orden:

$$
(\sigma+2) s^{\prime 3}-\alpha \eta^{\sigma+1} s^{\prime 2}=0
$$

de donde se obtiene $s=\left(\alpha /(\sigma+2)^{2}\right) \eta^{\sigma+2}$. Esta solución diverge exponencialmente para $\eta \rightarrow \infty$, por lo que el coeficiente $b$ ha de ser nulo.

Así pues, el desarrollo de la función $g$ para $\eta \rightarrow \infty$ es:

$$
g \simeq \frac{\alpha}{\sigma+1} \eta^{\sigma+1}+\frac{R e^{2 \sigma /(\sigma+2)} P(1)}{\alpha(1-2 \sigma)} \eta^{1-\sigma}+c \eta^{\sigma}
$$

donde $c$ es una constante libre.

La ecuación (3.31) se resuelve numéricamente partiendo de la condición (3.39) para $\eta \rightarrow \infty$, y buscando valores de $c$ y $\sigma$ para los que se cumplan las condiciones de contorno (3.32).

El problema (3.31) - (3.33) no tiene solución para $\sigma>1 / 2$. Para $\sigma \leq 1 / 2$ el segundo término del desarrollo (3.39) acopla con la segunda solución de $U$ en (3.27), que es la menos singular de las dos, mientras que la primera no es posible en la región exterior. Se obtiene que la ecuación sólo tiene solución cuando $\sigma=\sigma_{c} \approx 0,364$ (Higuera 1994).

\subsubsection{Estimación del ángulo de la lámina cónica}

La conservación de la cantidad de movimiento y del momento de la cantidad de movimiento de la lámina líquida anular que emerge del atomizador implica que, mientras las fuerzas aerodinámicas sean despreciables, la lámina tiende asintóticamente a un cono, y la velocidad de giro tiende a anularse, lejos del orificio de salida. Para un líquido sin viscosidad, el ángulo del cono puede determinarse sin necesidad de un análisis detallado de la evolución de la lámina aguas abajo de la cámara (Taylor 1948), pero no parece que esto sea posible para un líquido real con viscosidad. Para determinar dicho ángulo se empleará la aproximación que se muestra en las siguientes líneas: 
- Primero, se aplica la conservación de la cantidad de movimiento axial al volumen de control que comprende la lámina líquida entre el final del orificio de salida y una sección lejos aguas abajo de dicho orificio, donde la viscosidad ha uniformizado la velocidad a través de la lámina y la presión es igual a la del aire ( $p=0$ en variables adimensionales). Llamamos $r_{\infty_{1}}$ y $r_{\infty_{2}}$ a los radios de las superficies exterior e interior de la lámina líquida en esta sección, respectivamente.

$$
\int_{r_{0}}^{1} 2 \pi r u^{2} \mathrm{~d} r+\int_{r_{0}}^{1} 2 \pi r p \mathrm{~d} r=\int_{r_{\infty_{2}}}^{r_{\infty_{1}}} 2 \pi r u_{\infty}^{2} \mathrm{~d} r
$$

Teniendo en cuenta la conservación de masa:

$$
\int_{r_{0}}^{1} 2 \pi r u \mathrm{~d} r=\int_{r_{\infty_{2}}}^{r_{\infty_{1}}} 2 \pi r u_{\infty} \mathrm{d} r
$$

Sustituyendo (3.41) en (3.40):

$$
\int_{r_{0}}^{1} 2 \pi r u^{2} \mathrm{~d} r+\int_{r_{0}}^{1} 2 \pi r p \mathrm{~d} r=u_{\infty} \int_{r_{0}}^{1} 2 \pi r u \mathrm{~d} r
$$

De la anterior expresión se puede obtener la velocidad axial lejos aguas abajo en función de las variables a la salida del orificio:

$$
u_{\infty}=\frac{\phi_{c m}+\phi_{p}}{\phi_{m}}
$$

donde:

$$
\phi_{c m}=\int_{r_{0}}^{1} 2 \pi r u^{2} \mathrm{~d} r, \quad \phi_{p}=\int_{r_{0}}^{1} 2 \pi r p \mathrm{~d} r, \quad \phi_{m}=\int_{r_{0}}^{1} 2 \pi r u \mathrm{~d} r
$$

- Segundo, se integra la ecuación de la energía cinética del líquido sobre el mismo volumen de control:

$$
\int_{r_{0}}^{1} 2 \pi r\left[\frac{u^{2}+w^{2}}{2}+p\right] u \mathrm{~d} r+\Phi=\int_{r_{\infty_{2}}}^{r_{\infty_{1}}} 2 \pi r \frac{u_{\infty}^{2}+v_{\infty}^{2}}{2} u_{\infty} \mathrm{d} r
$$

donde $v_{\infty}$ es la velocidad radial del líquido lejos aguas abajo del orificio, escalada como la velocidad axial, y $\Phi$ es la disipación viscosa integrada sobre el volumen de control. Si se desprecia esta última cantidad y se sustituye la expresión (3.41) en (3.45):

$$
\int_{r_{0}}^{1} 2 \pi r\left[\frac{u^{2}+w^{2}}{2}+p\right] u \mathrm{~d} r=\frac{u_{\infty}^{2}+v_{\infty}^{2}}{2} \int_{r_{0}}^{1} 2 \pi r u \mathrm{~d} r
$$

De esta última expresión se puede obtener la velocidad en la dirección de la lámina cónica lejos aguas abajo en función de las variables a la salida del orificio:

$$
\sqrt{u_{\infty}^{2}+v_{\infty}^{2}}=\sqrt{\frac{2 \phi_{H}}{\phi_{m}}}
$$


donde:

$$
\phi_{H}=\int_{r_{0}}^{1} 2 \pi r\left[\frac{u^{2}+w^{2}}{2}+p\right] u \mathrm{~d} r
$$

- Finalmente, el coseno del semiángulo de la lámina cónica se obtiene de la relación entre la velocidad axial y la velocidad en la dirección de la lámina cónica lejos aguas abajo del orificio:

$$
\cos \theta=\frac{u_{\infty}}{\sqrt{u_{\infty}^{2}+v_{\infty}^{2}}}
$$

de donde:

$$
\theta=\arccos \frac{\phi_{c m}+\phi_{p}}{\sqrt{2 \phi_{m} \phi_{H}}}
$$

La aproximación (3.50) es similar a las aproximaciones propuestas por Datta \& Som (2000), Yule \& Chinn (2000) y Nouri-Borujerdi \& Kebriaee (2012).

Yule \& Chinn (2000) estiman los valores del semiángulo $\theta$ de la lámina cónica calculando los valores medios de las componentes de la velocidad obtenidas a lo largo de la salida:

$$
\bar{u}_{0}=\frac{2 \pi \int_{r_{0}}^{1} r u_{0} \mathrm{~d} r}{\pi\left(1-r_{0}^{2}\right)}, \quad \bar{v}_{0}=\frac{2 \pi \int_{r_{0}}^{1} r v_{0} \mathrm{~d} r}{\pi\left(1-r_{0}^{2}\right)}, \quad \bar{w}_{0}=\frac{2 \pi \int_{r_{0}}^{1} r w_{0} \mathrm{~d} r}{\pi\left(1-r_{0}^{2}\right)}
$$

luego:

$$
\theta=\arctan \frac{\sqrt{\bar{v}_{0}^{2}+\bar{w}_{0}^{2}}}{\bar{u}_{0}}
$$

La aproximación empleada por Datta \& Som (2000) y Nouri-Borujerdi \& Kebriaee (2012) es:

$$
\theta=\arccos \frac{\bar{u}_{0}}{\sqrt{\bar{u}_{0}^{2}+\bar{v}_{0}^{2}+\bar{w}_{0}^{2}}}
$$

con:

$$
\bar{u}_{0}=\frac{Q}{\pi\left(1-r_{0}^{2}\right)}, \quad \bar{v}_{0}=\frac{\int_{r_{0}}^{1} r u_{0} v_{0} \mathrm{~d} r}{\int_{r_{0}}^{1} r u_{0} \mathrm{~d} r}, \quad \bar{w}_{0}=\frac{\int_{r_{0}}^{1} r u_{0} w_{0} \mathrm{~d} r}{\int_{r_{0}}^{1} r u_{0} \mathrm{~d} r}
$$

\subsubsection{Simulación numérica}

El problema presenta una singularidad cuando $x \rightarrow 1+\ell_{2}$. Para tenerla en cuenta en el tratamiento numérico se ha usado la siguiente variable:

$$
\xi=\left(1+\ell_{2}-x\right)^{\kappa} \quad \text { con } \quad \kappa<\lambda \quad \text { tal que } \quad \frac{\partial}{\partial \xi}\left(u, w, p, r_{c}\right) \rightarrow 0 \quad \text { cuando } \quad \xi \rightarrow 0
$$

El problema se ha reescrito en términos de las variables $\xi$ y $\zeta=\left(R_{w}-r\right) /\left(R_{w}-r_{c}\right)$, que satisface $0<\zeta<1$. En términos de estas variables la solución es regular cuando $\xi \rightarrow 0$ si $\kappa$ es apropiado. 
Las ecuaciones que resultan tras este cambio de variable se han discretizado usando diferencias finitas. Se ha empleado una iteración pseudotransitoria, que consiste en añadir a las ecuaciones de cantidad de movimiento axial y azimutal los términos transitorios $\partial u / \partial t$ y $\partial w / \partial t$ respectivamente.

El dominio ha sido discretizado de tal forma que la concentración de nodos en las regiones donde se generen capas límite sea mayor, debido a los mayores gradientes presentes en estas zonas.

\subsection{Resultados}

En la figura 3.3 se muestran algunas características del flujo en el atomizador para $R e=2000, R_{s}=3, \ell_{1}=0, \ell_{2}=0,2, u_{c}=1$ y diferentes valores de la constante del atomizador $\Delta$ y del parámetro $\delta$ introducido en (3.13) que mide el espesor de la capa límite alrededor del núcleo de aire en la sección de entrada $x=-\ell_{1}$. Se representan secciones axiales de tubos de corriente (líneas continuas) e isobaras (líneas discontinuas).
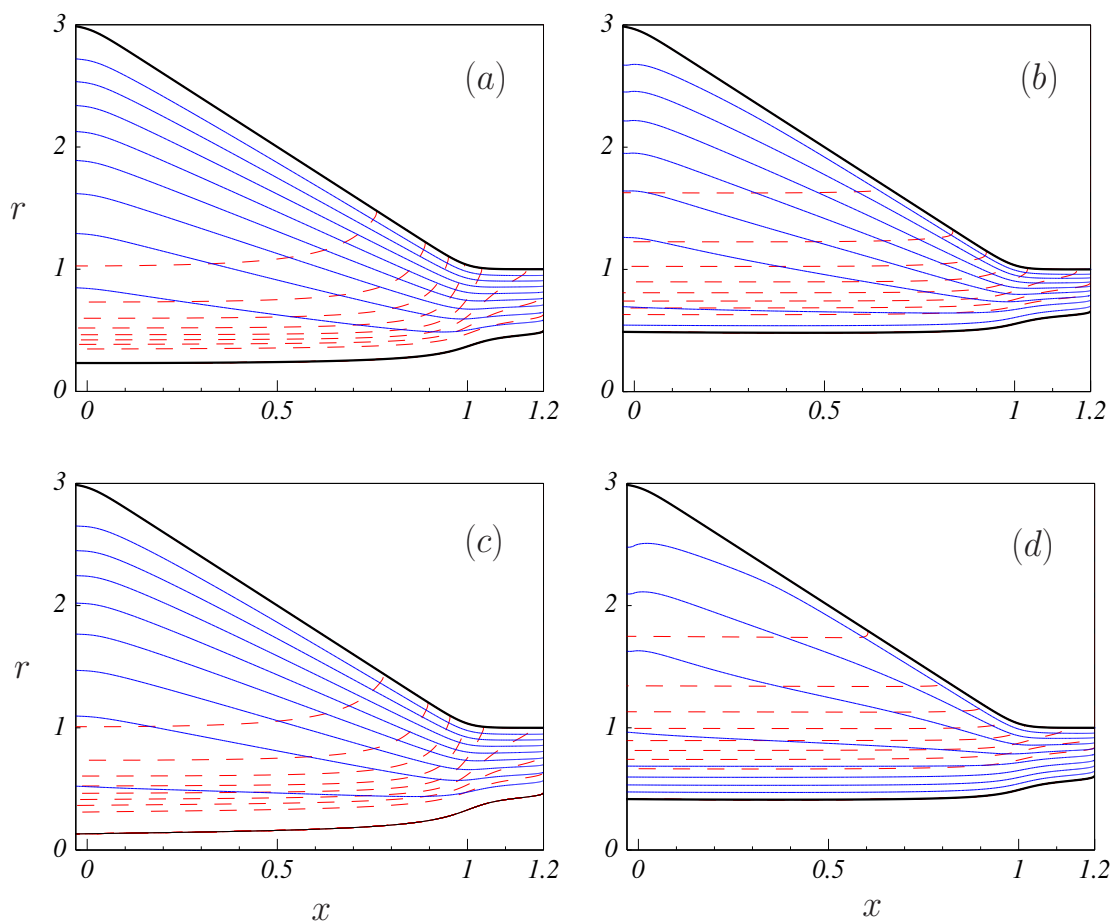

Figura 3.3 - Líneas de corriente (líneas continuas) e isobaras (líneas discontinuas) para: (a) $\Delta=1,25, \delta=0,1$; (b) $\Delta=0,4, \delta=0,1$; (c) $\Delta=1,25, \delta=0,245$; (d) $\Delta=0,3925$, $\delta=0,245$. Los valores de otros parámetros son: $R e=2000, R_{s}=3, \ell_{1}=0, \ell_{2}=0,2$, $u_{c}=1$. 
En buen acuerdo con los resultados numéricos y experimentales conocidos, las isobaras son prácticamente cilíndricas en el interior de la cámara, lo que indica un efecto dominante del giro del líquido cuya aceleración centrípeta produce la variación radial de presión. En las proximidades del orificio se genera un gradiente axial de presión que empuja al líquido fuera de la cámara. La velocidad axial es pequeña comparada con la velocidad azimutal excepto en la proximidad del orificio y en las capas límite alrededor de la pared convergente de la cámara y del núcleo de aire. El radio del núcleo de aire es prácticamente uniforme en el interior de la cámara y aumenta en el orificio de salida, debido a la disminución de presión en el mismo.

\subsubsection{Efecto de la constante del atomizador}

En la figura 3.4(a) se muestra el coeficiente de descarga, $c_{d}=Q /\left[2^{1 / 2} \pi\left(p_{0}-p_{a}\right)^{1 / 2}\right]$, en función de la constante del atomizador $\Delta$. La sobrepresión adimensional en la cámara, $p_{0}-p_{a}$, se ha tomado como $p_{0}-p_{a}=\max _{r}\left[p+\left(u^{2}+w^{2}\right) / 2\right]$ en la sección de entrada $x=-\ell_{1}$ pero fuera de la capa límite que rodea el núcleo de aire. Como puede observarse, el coeficiente de descarga calculado es ligeramente superior al que predice la teoría irrotacional, lo que se traduce en una sobrepresión en la cámara más pequeña que la obtenida por la teoría irrotacional para un caudal adimensional dado. Esto es un efecto del exceso de velocidad axial inducido por el bombeo de Ekman en las capas límite, que da cuenta de una parte del caudal sin requerir ninguna fuerza de presión en la cámara. La fracción del caudal que está en la capa límite aumenta cuando la constante del atomizador disminuye, por lo que la diferencia entre el coeficiente de descarga obtenido y el que predice la teoría irrotacional aumenta. La figura 3.4(a) también muestra que esta diferencia disminuye cuando lo hace $\delta$, pues entonces el espesor de la capa límite alrededor del núcleo de aire disminuye. Los cuadrados en esta figura representan resultados experimentales obtenidos por Dombrowski \& Hasson (1969) para $R_{s}=6, \ell_{1}=0, \ell_{2}=0,029$ (cuadrados llenos) y para $R_{s}=9, \ell_{1}=0, \ell_{2}=0,018$ (cuadrados vacíos). Los círculos llenos representan resultados experimentales obtenidos por Yule \& Widger (1996) para $R_{s}=6,54, \ell_{1}=4,94$, $\ell_{2}=1,18$, y los círculos vacíos por Yule \& Chinn (2000) para $R_{s}=3,31, \ell_{1}=4,69$, $\ell_{2}=1,05$. Los rombos son resultados numéricos obtenidos por Jeng et al. (1998) para $R_{s}=4,22, \ell_{1}=3,6, \ell_{2}=1,1, \mathrm{y}$ los asteriscos son resultados experimentales de Rizk \& Lefebvre (1985) para $R_{s}=4, \ell_{1}=0,33, \ell_{2}=1,3$. 
(a)

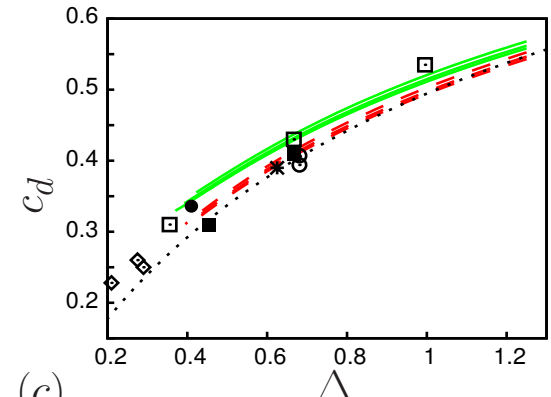

(c)

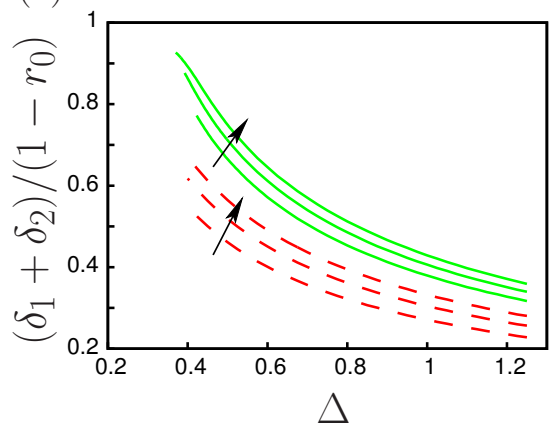

(b)

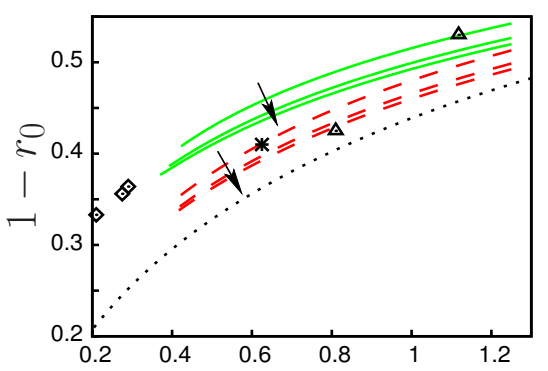

(d)

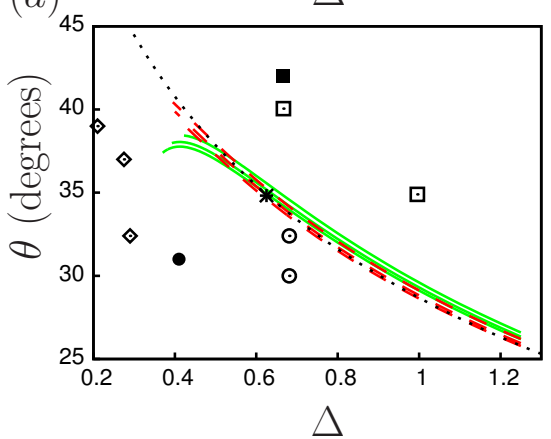

Figura 3.4 - Características del atomizador en función de la constante del atomizador $\Delta$ para $\delta=0,245$ (líneas continuas) y $\delta=0,1$ (líneas discontinuas), y $\ell_{2}=0,0,2,0,4$, incrementando como indican las flechas. Los valores de otros parámetros son: $R e=2000$, $R_{s}=3, \ell_{1}=0, u_{c}=1$. Las curvas punteadas muestran los resultados obtenidos por Taylor (1948). Los símbolos representan resultados experimentales y numéricos obtenidos por Dombrowski \& Hasson (1969) (cuadrados), Yule \& Widger (1996) (círculos rellenos), Yule \& Chinn (2000) (círculos vacíos), Halder et al. (2003) (triángulos), Jeng et al. (1998) (rombos) y Rizk \& Lefebvre (1985) (asteriscos). (a) Coeficiente de descarga $\left(c_{d}\right)$. (b) Espesor de la lámina líquida al final del orificio $\left(1-r_{0}\right)$. (c) Suma de los espesores de las capas límite al final del orificio escalada con el espesor local de la lámina líquida $\left(\left(\delta_{1}+\delta_{2}\right) /\left(1-r_{0}\right)\right)$. (d) Semiángulo de la lámina cónica $(\theta)$.

El valor calculado del espesor de la lámina líquida al final del orificio de salida se muestra en la figura 3.4(b) junto con la predicción de la teoría irrotacional, resultados numéricos obtenidos por Jeng et al. (1998) (rombos), y resultados experimentales obtenidos por Rizk \& Lefebvre (1985) (asteriscos) y Halder et al. (2003) (triángulos, con $R_{s}=4$ y los dos valores $\ell_{2}=0,49$ y $\left.\ell_{2}=0,97\right)$. El espesor de la lámina en el orificio de salida es mayor que el obtenido mediante la teoría irrotacional debido principalmente a la capa límite alrededor del núcleo de aire. Este mayor espesor aumenta cuando se incrementa $\delta$. Las distancias a la pared del orificio y a la interfaz entre el líquido y el aire donde $r w=0,99$ se han usado para definir los espesores de las capas límite, $\delta_{1}$ y $\delta_{2}$ respecti- 
vamente. La relación entre la suma de dichos espesores y el espesor de la lámina líquida al final del orificio se ha representado en la figura 3.4(c) en función de la constante del atomizador. Esta relación aumenta cuando la constante del atomizador disminuye, y las capas límite cubren gran parte del espesor de la lámina líquida para el valor más pequeño de la constante del atomizador para el cual se ha calculado una solución cuando $\delta=0,245$. Esto está de acuerdo con las estimaciones de Taylor (1948) y Dumouchel et al. (1992). La presencia de estas capas límite también da lugar a valores del radio del núcleo de aire en el interior de la cámara más pequeños que los obtenidos por la teoría irrotacional. Este radio aumenta cuando $\delta$ disminuye, y también cuando la constante del atomizador disminuye, pues disminuye la sobrepresión en la cámara.

El valor reducido de $r w$ en la capa límite alrededor del núcleo de aire tiene un efecto importante en los resultados porque es en esta región donde se produce el mayor incremento radial de presión de acuerdo con la teoría irrotacional, debido a los menores valores del radio. Debido a ello la concentración de isobaras en la proximidad del núcleo de aire mostradas en la figura 3.3(a), para $\delta=0,1$, cuando la capa con velocidad de giro reducida es fina, puede compararse con el mayor espaciado que muestra la figura 3.3(c), para $\delta=0,245$, donde existe una región más ancha de baja presión alrededor del núcleo de aire. El espesor de la capa límite al final del orificio de salida y en el interior de la cámara aumenta cuando la constante del atomizador disminuye, lo que merma la capacidad del atomizador de generar la sobrepresión requerida. Por ejemplo, el ángulo que forman las isobaras con las líneas de corriente en las proximidades del orificio de salida es mayor en las figuras 3.3(a,c) que en las figuras 3.3(b,d), lo que sugiere que la fuerza de presión es más eficiente para acelerar el líquido en el primer caso, con mayor valor de la constante del atomizador, que en el segundo.

El valor aproximado (3.50) del semiángulo del cono se muestra en la figura 3.4(d) en función de la constante del atomizador. El resultado es próximo a la predicción de la teoría irrotacional, en la que no hay disipación viscosa, excepto para valores pequeños de la constante del atomizador, para los cuales (3.50) cae por debajo de la predicción irrotacional. La diferencia se debe al aumento del espesor de las capas límite de velocidad azimutal reducida cuando la constante del atomizador disminuye. La conservación de la constante de Bernoulli en el flujo irrotacional se traduce aproximadamente en una conversión de la velocidad azimutal al final del orificio de salida en velocidad radial lejos aguas abajo. Debido a que en las capas límite se reduce la velocidad azimutal, la velocidad radial aguas abajo del orificio de salida también se reduce, por lo que el semiángulo de la lámina cónica disminuye. 


\subsubsection{Efectos de otros parámetros geométricos $\left(R_{s}, \ell_{1}, \ell_{2}\right)$}

Los efectos de otros parámetros geométricos $\left(R_{s}, \ell_{1}, \ell_{2}\right)$ en el coeficiente de descarga, el espesor de la lámina líquida al final del orificio de salida y el ángulo de la lámina cónica aguas abajo de dicho orificio se muestran en la figura 3.5, donde dichas características del flujo se han escalado con los valores calculados por Taylor (1948) incluidos en la Sección 2.3. Las variaciones calculadas son pequeñas comparadas con las debidas a la variación de la constante del atomizador $\Delta$ discutidas anteriormente. Estos resultados están de acuerdo con cálculos previos (Sakman et al. 2000) y resultados experimentales (Dombrowski \& Hasson 1969; Rizk \& Lefebvre 1985; Suyari \& Lefebvre 1986), aunque las variaciones del coeficiente de descarga $c_{d}$, del espesor de la lámina líquida al final del orificio de salida $1-r_{0}$ y del ángulo $\theta$ del cono que forma la lámina líquida mostradas en la figura 3.5 son significativamente más pequeñas que las variaciones calculadas por Sakman et al. (2000) para $\Delta=0,3$. Esto se debe probablemente a diferencias importantes en el flujo interno, que no pueden ser calculadas mediante la aproximación cuasi-cilíndrica para la combinación de valores grandes de $\ell_{1}$ y valores pequeños de $\Delta$ empleada por estos autores, pues esta combinación parece dar lugar a inestabilidad centrífuga y alta recirculación.

Debido a la forma en que se modela la velocidad axial a la entrada en (3.12) y (3.13), la fracción del flujo en la capa límite alrededor del núcleo de aire aumenta cuando el radio $R_{s}$ disminuye, manteniendo $\delta$ y $u_{c}$ constantes. La evolución aguas abajo de esta capa también depende de $R_{s}$. La velocidad axial en la cámara no es mucho más pequeña que la velocidad axial en la capa límite para los valores más pequeños de $R_{s}$ en la figura 3.5, luego las dos aumentan más o menos al mismo ritmo bajo la acción de la fuerza de presión requerida para mantener el caudal constante en la cámara convergente, y el espesor de la capa límite decrece suavemente con la distancia en el sentido de la corriente. Sin embargo, cuando el radio $R_{s}$ aumenta, la velocidad axial es mucho más pequeña en la cámara que en la capa límite. En este caso, una fuerza de presión pequeña, que tiene poco efecto en la capa límite, basta para acelerar el flujo en casi toda la cámara. La caída de presión más grande ocurre en las proximidades del orificio de salida, donde el espesor de la capa límite disminuye rápidamente. Resulta que el espesor de la capa límite al final del orificio de salida, y la fracción del caudal que se encuentra en dicha capa, aumenta cuando el radio $R_{s}$ disminuye hacia el valor mínimo mostrado en la figura 3.5. La figura 3.5(b) muestra que el espesor de la lámina líquida a la salida tambien aumenta, pero menos marcadamente que el espesor de la capa límite, luego la relación entre ambos aumenta, como muestra la figura 3.5(c). Debido a la velocidad de giro reducida en la capa límite 
alrededor del núcleo de aire, el incremento radial de presión a través de la lámina líquida al final del orificio de salida decrece con el radio $R_{s}$. Debido a ello decrece la sobrepresión requerida en la cámara para generar el flujo, por lo que aumenta el coeficiente de descarga, como muestra la figura 3.5(a). La figura 3.5(d) muestra que el ángulo de la lámina cónica también disminuye con $R_{s}$ para valores pequeños de este parámetro, lo cual es debido al incremento de la fracción del flujo que alcanza el final del orificio de salida con una velocidad de giro reducida. Todas estas variaciones aquí discutidas para valores pequeños de $R_{s}$ se hacen menores cuando se disminuye el espesor de la capa límite alrededor del núcleo de aire disminuyendo el parámetro $\delta$.

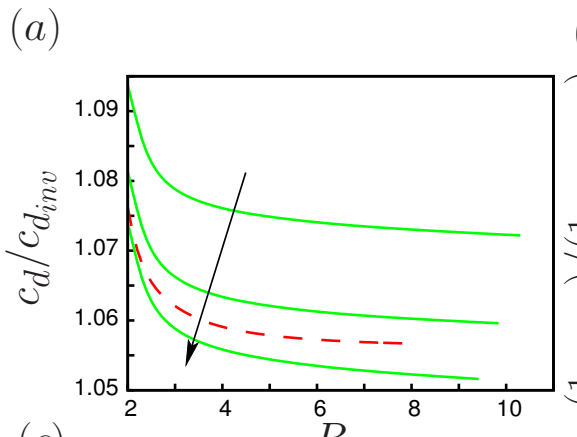

(c)

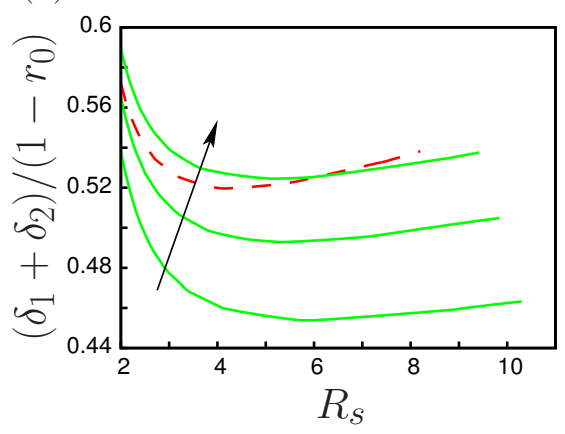

(b)
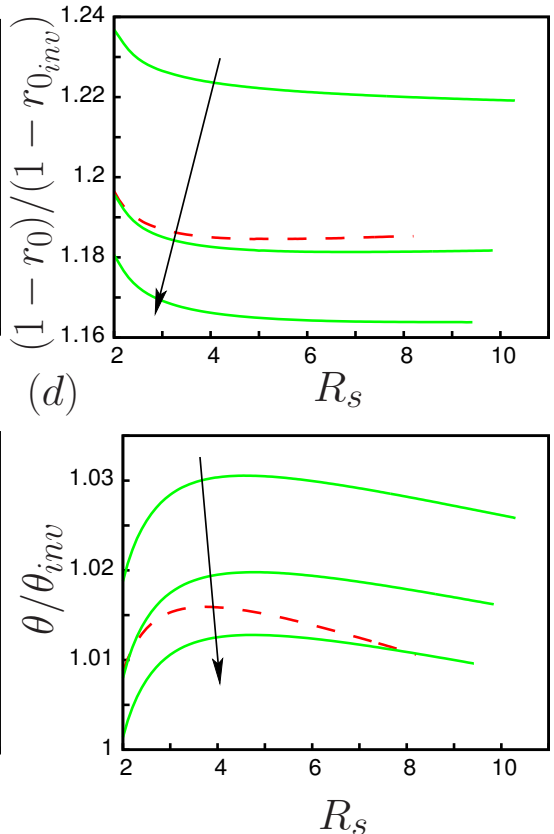

Figura 3.5 - Características del atomizador en función del radio de la cámara $R_{s}$ para $\ell_{1}=0$ y $\ell_{2}=0,0,2,0,4$, incrementando como indican las flechas (líneas continuas), y para $\ell_{1}=0,5$ y $\ell_{2}=0,2$ (líneas discontinuas). Los valores de otros parámetros son: $R e=2000, \Delta=0,75, \epsilon=0,2, u_{c}=1, \delta=0,245$. (a) Coeficiente de descarga escalado con el valor calculado por Taylor $(1948),\left(c_{d} / c_{d_{i n v}}\right)$. (b) Espesor de la lámina líquida al final del orificio escalada con el valor calculado por Taylor $(1948),\left(\left(1-r_{0}\right) /\left(1-r_{0_{i n v}}\right)\right)$. (c) Suma de los espesores de las capas límite al final del orificio escalada con el espesor local de la lámina líquida $\left(\left(\delta_{1}+\delta_{2}\right) /\left(1-r_{0}\right)\right)$. (d) Semiángulo de la lámina cónica escalado con el valor calculado por Taylor (1948), $\left(\theta / \theta_{\text {inv }}\right)$. 
La capa límite alrededor de la pared convergente de la cámara juega un papel importante para valores altos del radio $R_{s}$. El espesor de esta capa límite aumenta casi linealmente con $R_{s}$ (Binnie \& Harris 1950; Taylor 1950a; Binnie et al. 1957; Burggraf et al. 1971; Bloor \& Ingham 1977), dando lugar al suave incremento en la relación entre la suma de los espesores de las capas límite en el orificio de salida y el espesor de la lámina líquida en el mismo mostrada en la figura 3.5(c), y a la disminución del ángulo de la lámina cónica indicada en la figura 3.5(d), cuando aumenta el valor de $R_{s}$.

La velocidad axial del flujo en la cámara disminuye cuando aumenta el radio $R_{s}$, lo que hace que el flujo sea más sensible a las pequeñas variaciones de presión generadas cuando la capa límite sobre la pared entra en la parte convergente de la cámara, donde el bombeo de Ekman da lugar a altas velocidades. Cuando $R_{s}$ aumenta aparece recirculación en esta capa límite, como se muestra en la figura 3.6(b). La aproximación cuasi-cilíndrica puede dar cuenta de una recirculación moderada, pero falla cuando el tamaño de la región de recirculación aumenta. Esto ocurre en los puntos finales de las curvas de la figura 3.5, que marcan el límite a partir del cual no se puede usar esta formulación simplificada.

La figura 3.5 también muestra los efectos de las longitudes adimensionales del orificio, $\ell_{2}$, y de la región casi cilíndrica de la cámara, $\ell_{1}$, en las características del atomizador. El efecto de ambos parámetros es moderado. El efecto de $\ell_{2}$ se puede entender teniendo en cuenta que el espesor de la capa límite en el orificio de salida aumenta con $\ell_{2}$, como indica la figura 3.5(c). Esto aumenta la región de velocidad de giro reducida al final del orificio, lo que da lugar a la disminución del ángulo de la lámina cónica que se ve en la figura 3.5(d). La presión en la cámara debe aumentar para mantener el caudal constante cuando el espesor de la capa límite y la fuerza de fricción aumentan, dando lugar a la disminución del coeficiente de descarga que aparece en la figura 3.5(a). El espesor total de la lámina líquida al final del orificio de salida disminuye cuando aumenta $\ell_{2}$, como se ve en la figura 3.5(b), a pesar del incremento del espesor de la capa límite. Esto es un efecto de la mayor fuerza de presión que actúa en el líquido fuera de la capa límite, que aumenta la velocidad al final del orificio. Todos estos resultados están de acuerdo con los resultados obtenidos por Sakman et al. (2000) y las referencias incluidas en su estudio.

Como se ve comparando la línea continua central con la línea discontinua en las figuras 3.5(c,d), el espesor de la capa límite de la pared (donde la velocidad de giro es reducida) aumenta, y el ángulo de la lámina cónica disminuye, cuando aumenta $\ell_{1}$. El coeficiente de descarga disminuye ligeramente cuando aumenta $\ell_{1}$ de 0 a 0,5 , aunque esta tendencia cambia para valores altos de $\ell_{1}$ de acuerdo con los resultados obtenidos por Sakman et al. (2000). De cualquier manera, el incremento del espesor de la capa límite al final del orificio 
de salida supera la pequeña variación de velocidad fuera de la capa límite y da lugar al ligero aumento del espesor de la lámina líquida con $\ell_{1}$ mostrado en la figura 3.5(b).
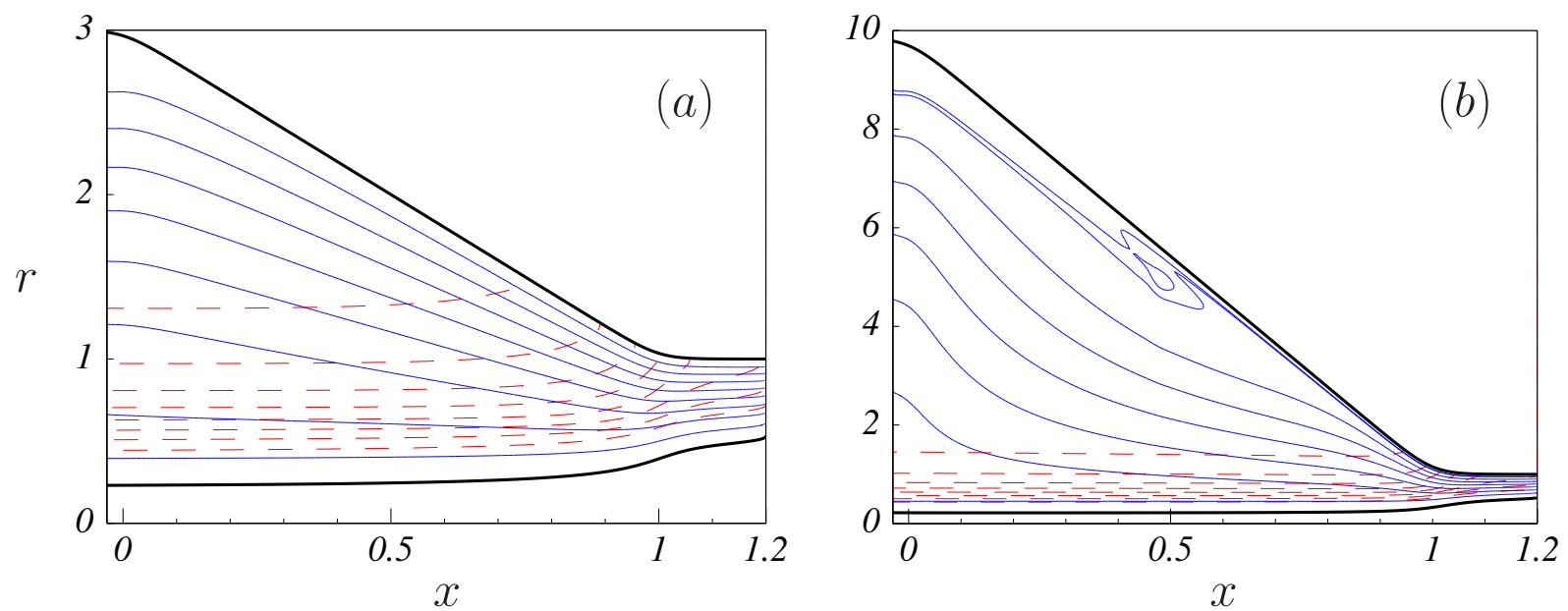

Figura 3.6 - Líneas de corriente (líneas continuas) e isobaras (líneas discontinuas) para: (a) $R_{s}=3$ y (b) $R_{s}=9,84$. Los valores de otros parámetros son: $R e=2000$, $\Delta=0,75, \ell_{1}=0, \ell_{2}=0,2, u_{c}=1, \delta=0,245$.

\subsubsection{Efectos del número de Reynolds}

Se han realizado cálculos adicionales variando el número de Reynolds dentro del rango 500 - 5000. En buen acuerdo con resultados experimentales y numéricos conocidos (Dombrowski \& Hasson 1969; Jeng et al. 1998; Halder et al. 2003), estos cálculos muestran que el espesor de las capas límite al final del orificio de salida aumenta cuando el número de Reynolds disminuye, pero el efecto del número de Reynolds en el coeficiente de descarga, el espesor de la lámina líquida y el ángulo del cono que forma la misma a la salida del atomizador es pequeño.

Wimmer \& Brenn (2013) indicaron que, para valores moderados de la viscosidad del líquido y para una geometría del atomizador y una diferencia de presión dadas, una mayor viscosidad del líquido resulta en un mayor caudal suministrado. En términos adimensionales, cuando la diferencia de presión $p_{0}-p_{a}$ se usa como variable de control en lugar del caudal $Q$, la viscosidad del líquido aparece a través del número de Reynolds modificado, $\widetilde{R e}=\pi\left[2 \rho\left(p_{0}-p_{a}\right)\right]^{1 / 2} R_{0}^{2} /(L \mu)=4 R e \Delta / c_{d}$. Los resultados de Wimmer \& Brenn (2013) vienen a decir que el coeficiente de descarga $c_{d}$ disminuye cuando aumenta el Reynolds modificado $\widetilde{R e}$. Para comprobar que la aproximación cuasi-cilíndrica reproduce estos resultados, se han realizado simulaciones en las cuales se ha variado el Reynolds $R e$ manteniendo constante $R e \delta^{2}=12$ y fijando los otros parámetros adimensionales a los 
valores $\Delta=0,7, R_{s}=3, \ell_{1}=0, \ell_{2}=0,2$ y $u_{c}=1$. Los resultados obtenidos de estas simulaciones se recogen en la tabla 3.1 .

\begin{tabular}{ccc}
$R e$ & $c_{d}$ & $\widetilde{R e}$ \\
\hline 14000 & 0.4468 & 87735 \\
18000 & 0.4434 & 113667 \\
22000 & 0.4410 & 139683 \\
26000 & 0.4390 & 165831 \\
\hline
\end{tabular}

Tabla 3.1 - Valores del coeficiente de descarga $c_{d}$ en función del número de Reynolds Re.

Se muestra que el coeficiente de descarga $c_{d}$ disminuye cuando aumenta el Reynolds modificado $\widetilde{R e}$, de acuerdo con los resultados obtenidos por Wimmer \& Brenn (2013). También de acuerdo con los resultados de estos autores, al aumentar el Reynolds modificado $\widetilde{R e}$, el radio del núcleo de aire al final del orificio aumenta, y la velocidad axial en la superficie del líquido disminuye. Sin embargo, la velocidad axial media calculada, $c_{d} /\left(1-r_{0}^{2}\right)$, aumenta ligeramente con $\widetilde{R e}$. Cousin \& Nuglish (2001) y Moon, Abo-Serie \& Bae (2010) observaron que el número de Reynolds deja de tener efecto cuando aumenta a valores muy por encima de los empleados en estas simulaciones o en el trabajo de Wimmer \& Brenn (2013). 


\section{Capítulo 4}

\section{Forma y estabilidad de la lámina líquida}

Cuando la lámina líquida anular alcanza el orificio de salida comienza a diverger radialmente aguas abajo del mismo, donde ya no está confinada por las paredes de la cámara, y acaba rompiéndose en ligamentos y posteriormente en gotas a cierta distancia de la cámara. Las propiedades de la lámina a la salida del orificio están determinadas por la solución en el interior de la cámara, la cual es función del caudal $Q$ (o de la diferencia de presiones $p_{0}-p_{a}$, siendo $p_{0}$ la presión de remanso a la que se inyecta el líquido y $p_{a}$ la presión del gas del ambiente) y de la geometría del atomizador. Al aumentar la distancia al atomizador, en tanto el efecto de la tensión superficial se mantenga despreciable, la lámina toma una forma cónica. La presión del líquido en la lámina tiende entonces a la presión ambiente, $p_{a}$, la componente azimutal de la velocidad tiende a cero al crecer el radio de la lámina, y la velocidad del líquido tiende al valor $U_{\infty}=\sqrt{2\left(p_{0}-p_{a}\right) / \rho}$, donde $\rho$ es la densidad del líquido.

La fragmentación de una lámina líquida para producir un spray de gotas es un proceso que ocurre en varias etapas, generándose primero ligamentos que después se rompen en gotas, pero no existe una descripción completa del mismo que muestre las relaciones entre las inestabilidades responsables de cada etapa. Es sabido que la lámina oscila lateralmente, como una bandera bajo la acción del viento, cuando la velocidad del líquido relativa al medio que lo rodea es suficientemente alta. La oscilación lateral se debe a una inestabilidad de Kelvin-Helmholtz, con un amplio rango de longitudes de onda inestables (Taylor 1960; Huang 1970; Lin 2003). Squire (1953), York, Stubbs \& Tek (1953), Hagerty \& Shea (1955) y Lin (2003) presentaron análisis lineales de esta inestabilidad para una lámina en movimiento entre dos fases en reposo, mientras que Asare, Takahashi \& Hoffman (1981) y 
Villermaux \& Clanet (2002) mostraron que los resultados de estos análisis están en buen acuerdo con lo observado en sus experimentos. Más recientemente, Bremond, Clanet \& Villermaux (2007) han llevado a cabo experimentos controlados en los que la lámina se fuerza con una frecuencia y una amplitud conocidas, obteniendo oscilaciones nítidas que permiten estudiar el crecimiento de las perturbaciones y su efecto en etapas posteriores de la fragmentación de la lámina. Este efecto ha sido objeto de controversia. York, Stubbs \& Tek (1953), Fraser et al. (1962) y Dombrowski \& Johns (1963), entre otros, sostienen que la rotura se debe al crecimiento de perturbaciones alineadas con el borde de la lámina, con un espaciado igual a la mitad de la longitud de onda más inestable de la inestabilidad de Kelvin-Helmholtz, pero esta propuesta no explica la existencia de ligamentos perpendiculares al borde de la lámina, que se observan frecuentemente (Mansour \& Chigier 1990; Lozano, García-Olizares \& Dopazo 1998; Kim \& Sirignano 2000; Park et al. 2004). Bremond, Clanet \& Villermaux (2007) muestran, apoyándose en sus experimentos y en el trabajo previo de Villermaux \& Clanet (2002), que las aceleraciones transitorias del líquido perpendiculares a la lámina, provocadas por la inestabilidad oscilatoria de Kelvin-Helmholtz, son capaces de excitar una inestabilidad secundaria de Rayleigh-Taylor cuando la amplitud de las ondas y su velocidad relativa al líquido superan ciertos valores. Esta inestabilidad secundaria da lugar a variaciones azimutales del espesor de la lámina y es la causa de los ligamentos longitudinales mencionados antes. Observan que las escalas de longitud y tiempo deducidas de este mecanismo permiten explicar el tamaño de la lámina y su variación con el número de Weber observada experimentalmente. Muestran asímismo que la distribución de tamaños de gotas del spray resultante de la rotura de estos ligamentos podría predecirse si se dispusiese de información adicional sobre el número de ligamentos que aparecen en el borde de la lámina.

En este capítulo estudiaremos la estructura estacionaria de la lámina generada a la salida de la cámara de un atomizador, su inestabilidad primaria (de Kelvin-Helmholtz) y las condiciones que dan lugar a la inestabilidad secundaria de Rayleigh-Taylor.

\subsection{Lámina líquida estacionaria}

Empezaremos calculando la forma estacionaria de la lámina. La velocidad axial del líquido a la salida de la cámara del atomizador es $u_{0} \sim U_{\infty}$, mientras que el espesor de la lámina anular puede considerarse del orden del radio $R_{0}$ del orificio de salida. El efecto de la tensión superficial en esta región es pequeño porque el número de Weber

$$
W=\frac{\rho U_{\infty}^{2} R_{0}}{\sigma}
$$


es grande. Esta es la condición que permite la formación de una lámina líquida. Sin embargo, el efecto de la tensión superficial crece cuando la lámina diverge, y llega a cambiar la forma cónica de la lámina a cierta distancia del inyector (si la lámina no se rompe antes). Sean $r_{c}$ y $h_{c}$ los valores característicos de la distancia al inyector y el espesor de la lámina en la región donde el efecto de la tensión superficial es importante. Estas magnitudes se pueden estimar como sigue:

1. Teniendo en cuenta que la velocidad del líquido se mantiene de orden $U_{\infty}$, aunque cambia de valor entre la salida del atomizador y esta región lejana, la ecuación de continuidad proporciona la relación $r_{c} h_{c} \sim R_{0}^{2}$.

2. El salto de presión a través de cada superficie de la lámina debido a la tensión superficial es de orden $\sigma / r_{c}$, y la condición de que la fuerza resultante, perpendicular a la lámina, cause variaciones apreciables del flujo de cantidad de movimiento en la lámina proporciona la relación $\rho U_{\infty}^{2} h_{c} / r_{c} \sim \sigma / r_{c}$.

De estas dos relaciones se obtiene:

$$
\frac{r_{c}}{R_{0}} \sim W \quad \text { y } \quad \frac{h_{c}}{R_{0}} \sim \frac{1}{W}
$$

de modo que el número de Weber local, $W e=\rho U_{\infty}^{2} h / \sigma$, es de orden unidad en esta región.

La componente azimutal de la velocidad del líquido a la salida del atomizador es del orden de la componente axial $\left(\Gamma / R_{0} \sim u_{0}\right)$. Sin embargo, como la circulación se conserva en la evolución de las líneas fluidas cerradas que rodean al eje de simetría, la componente azimutal de la velocidad es sólo de orden $U_{\infty} / W$ a distancias de orden $r_{c}$ del atomizador, y la variación de presión a través de la lámina debida al giro del líquido es de orden $\rho\left(\Gamma / r_{c}\right)^{2} h_{c} / r_{c} \sim\left(\sigma / r_{c}\right) / W^{2}$, que es pequeña frente a la debida a la tensión superficial.

Usaremos coordenadas esféricas con origen en el vértice aparente de la lámina cónica que se obtiene sin tensión superficial, de forma que $r$ y $\theta$ representan la distancia a este origen y el ángulo con el eje de simetría, respectivamente. Escribiremos la ecuación de la superficie media de la lámina (la línea media en un plano meridional) como $\theta=\theta_{c}(r)$, mientras que $\theta=\theta_{c}+\Delta \theta_{s_{ \pm}}$denotarán las superficies exterior e interior de dicha lámina (ver figura 4.1). 

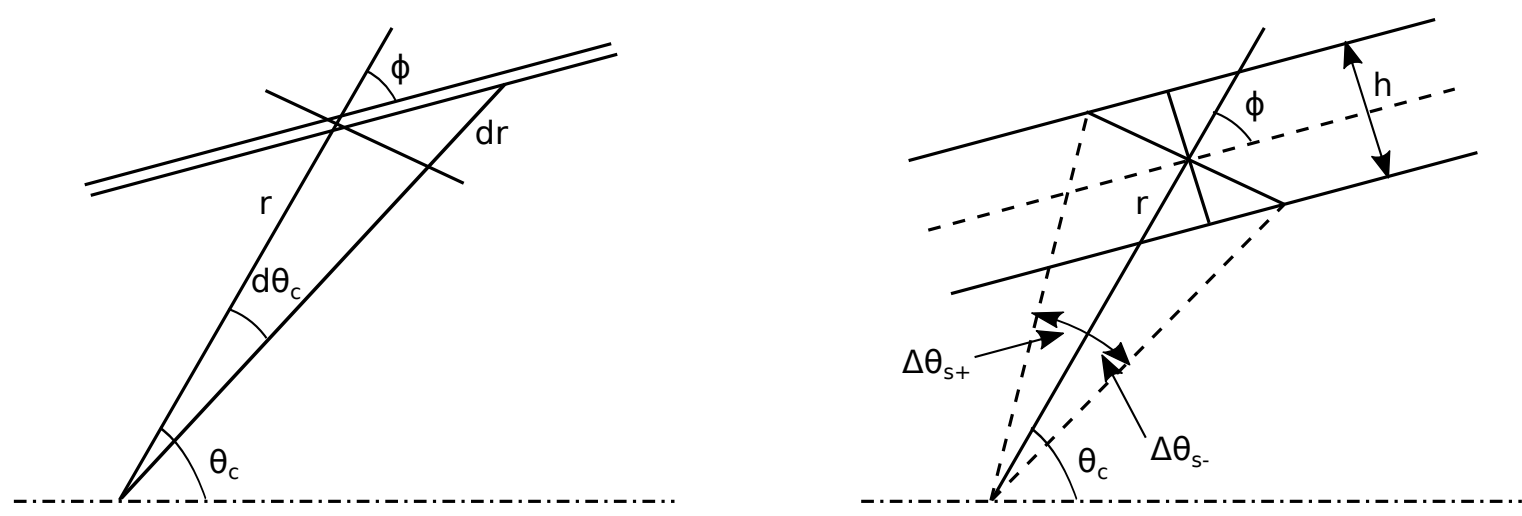

Figura 4.1 - Esquema de los parámetros usados para analizar la forma de la lámina líquida.

Llamamos $\phi$ al ángulo que forma la tangente a la línea media con la dirección radial local (ver figura 4.1). Así pues:

$$
\tan \phi=-\frac{r \mathrm{~d} \theta_{c}}{\mathrm{~d} r}
$$

El espesor de la lámina $h$ en función del ángulo $\phi$ es:

$$
\frac{h}{2}=r \Delta \theta_{s} \cos \phi
$$

de manera que:

$$
h=2 r \Delta \theta_{s}\left[1+r^{2}\left(\frac{\mathrm{d} \theta_{c}}{\mathrm{~d} r}\right)^{2}\right]^{-1 / 2}
$$

El problema a resolver es un problema axilsimétrico gobernado por la ecuación de Laplace que, en coordenadas esféricas, es la siguiente:

$$
\frac{1}{r^{2}} \frac{\partial}{\partial r}\left(r^{2} \frac{\partial \varphi_{s t}}{\partial r}\right)+\frac{1}{r^{2} \sin \theta} \frac{\partial}{\partial \theta}\left(\sin \theta \frac{\partial \varphi_{s t}}{\partial \theta}\right)=0
$$

donde $\varphi_{s t}$ es el potencial de velocidades del líquido en régimen estacionario, tal que las velocidades en dirección radial y angular son, respectivamente, $u=\partial \varphi_{s t} / \partial r$ y $v=$ $r^{-1} \partial \varphi_{s t} / \partial \theta$. La presión en régimen estacionario en el líquido, $p_{s t}$, viene dada por la ecuación de Euler-Bernoulli:

$$
\frac{1}{2}\left[\left(\frac{\partial \varphi_{s t}}{\partial r}\right)^{2}+\left(\frac{1}{r} \frac{\partial \varphi_{s t}}{\partial \theta}\right)^{2}+\left(\frac{\Gamma}{2 \pi r \sin \theta}\right)^{2}\right]+\frac{p_{s t}}{\rho}=\frac{1}{2} U_{\infty}^{2}+\frac{p_{a}}{\rho}
$$

Las condiciones de contorno cinemáticas en las superficies de la lámina líquida son:

$$
r \frac{\partial \theta_{c}}{\partial r} \frac{\partial \varphi_{s t}}{\partial r}=\frac{1}{r} \frac{\partial \varphi_{s t}}{\partial \theta} \quad \text { en } \quad \theta=\theta_{c}+\Delta \theta_{s_{s t \pm}}
$$


donde $\theta_{c}+\Delta \theta_{s_{s t \pm}}$ es la posición angular de las superficies exterior e interior de la lámina. Las condiciones de contorno dinámicas en las mismas son:

$$
p_{s t}-p_{a}=\sigma \nabla \cdot \mathbf{n}_{ \pm} \quad \text { en } \quad \theta=\theta_{c}+\Delta \theta_{s_{s t \pm}}
$$

donde $\mathbf{n}_{ \pm}$es el vector normal a cada una de esas superficies, orientado hacia el gas, y cuya expresión es la siguiente:

$$
\mathbf{n}_{ \pm}= \pm\left(-r \frac{\partial \theta_{c}}{\partial r}, 1\right)\left[1+r^{2}\left(\frac{\partial \theta_{c}}{\partial r}\right)^{2}\right]^{-1 / 2}
$$

Escribiendo $\theta=\theta_{c}+W^{-2} \tilde{\theta}$, con $\tilde{\theta}=O(1)$, y adimensionalizando $r$ con $W R_{0}$, el potencial de velocidades con $W U_{\infty} R_{0}$ y $p-p_{a}$ con $\rho U_{\infty}^{2}$, las ecuaciones (4.4) - (4.7) quedan en variables adimensionales de la siguiente manera:

$$
\begin{aligned}
& W^{4}\left[\left(\frac{\mathrm{d} \theta_{c}}{\mathrm{~d} r}\right)^{2}+\frac{1}{r^{2}}\right] \frac{\partial^{2} \varphi_{s t}}{\partial \tilde{\theta}^{2}}-W^{2}\left[\frac{1}{r^{2}} \frac{\mathrm{d}}{\mathrm{d} r}\left(r^{2} \frac{\mathrm{d} \theta_{c}}{\mathrm{~d} r}\right) \frac{\partial \varphi_{s t}}{\partial \tilde{\theta}}+2 \frac{\mathrm{d} \theta_{c}}{\mathrm{~d} r} \frac{\partial^{2} \varphi_{s t}}{\partial r \partial \tilde{\theta}}-\frac{\cot \theta_{c}}{r^{2}} \frac{\partial \varphi_{s t}}{\partial \tilde{\theta}}\right] \\
& +\frac{1}{r^{2}} \frac{\partial}{\partial r}\left(r^{2} \frac{\partial \varphi_{s t}}{\partial r}\right)-\frac{\tilde{\theta}}{r^{2} \sin ^{2} \theta_{c}} \frac{\partial \varphi_{s t}}{\partial \tilde{\theta}}+O\left(W^{-2}\right)=0 \\
& W^{4} \frac{1}{2}\left[\left(\frac{\mathrm{d} \theta_{c}}{\mathrm{~d} r}\right)^{2}+\frac{1}{r^{2}}\right]\left(\frac{\partial \varphi_{s t}}{\partial \tilde{\theta}}\right)^{2}-W^{2} \frac{\mathrm{d} \theta_{c}}{\mathrm{~d} r} \frac{\partial \varphi_{s t}}{\partial r} \frac{\partial \varphi_{s t}}{\partial \tilde{\theta}}+\left[\frac{1}{2}\left(\frac{\partial \varphi_{s t}}{\partial r}\right)^{2}+p_{s t}-\frac{1}{2}\right] \\
& +W^{-2} \frac{\gamma^{2}}{2 r^{2} \sin ^{2} \theta_{c}}-W^{-4} \frac{\gamma^{2} \tilde{\theta} \cos \theta_{c}}{r^{2} \sin ^{3} \theta_{c}}+O\left(W^{-6}\right)=0 \\
& W^{2}\left[\left(\frac{\mathrm{d} \theta_{c}}{\mathrm{~d} r}\right)^{2}+\frac{1}{r^{2}}\right] \frac{\partial \varphi_{s t}}{\partial \tilde{\theta}}+\frac{\mathrm{d} \theta_{c}}{\mathrm{~d} r}\left(\frac{\partial \tilde{\theta}_{s_{s t \pm}}}{\partial r} \frac{\partial \varphi_{s t}}{\partial \tilde{\theta}}-\frac{\partial \varphi_{s t}}{\partial r}\right)-W^{-2} \frac{\partial \tilde{\theta}_{s_{s t \pm}}}{\partial r} \frac{\partial \varphi_{s t}}{\partial r}=0 \\
& \text { en } \quad \theta=\theta_{c}+W^{-2} \tilde{\theta}_{s_{s t \pm}} \\
& p_{s t} \pm W^{-2}\left[\frac{1}{r^{2}} \frac{\partial}{\partial r}\left(\frac{r^{3} \frac{\mathrm{d} \theta_{c}}{\mathrm{~d} r}}{\left(1+r^{2}\left(\frac{\mathrm{d} \theta_{c}}{\mathrm{~d} r}+W^{-2} \frac{\partial \tilde{\theta}_{s_{s t \pm}}}{\partial r}\right)^{2}\right)^{1 / 2}}\right)-\frac{\frac{\cot \theta_{c}}{r}}{\left(1+r^{2}\left(\frac{\mathrm{d} \theta_{c}}{\mathrm{~d} r}+W^{-2} \frac{\partial \tilde{\theta}_{s t \pm \pm}}{\partial r}\right)^{2}\right)^{1 / 2}}\right] \\
& \pm W^{-4}\left[\frac{1}{r^{2}} \frac{\partial}{\partial r}\left(\frac{r^{3} \frac{\partial \tilde{\theta}_{s t t \pm}}{\partial r}}{\left(1+r^{2}\left(\frac{\mathrm{d} \theta_{c}}{\mathrm{~d} r}+W^{-2} \frac{\partial \tilde{\theta}_{s_{s t \pm}}}{\partial r}\right)^{2}\right)^{1 / 2}}\right)+\frac{\frac{\tilde{\theta}_{s_{s t \pm}}}{r \sin ^{2} \theta_{c}}}{\left(1+r^{2}\left(\frac{\mathrm{d} \theta_{c}}{\mathrm{~d} r}+W^{-2} \frac{\partial \tilde{\theta}_{s t \pm}}{\partial r}\right)^{2}\right)^{1 / 2}}\right] \\
& +O\left(W^{-6}\right)=0 \quad \text { en } \quad \theta=\theta_{c}+W^{-2} \tilde{\theta}_{s_{s t \pm}}
\end{aligned}
$$


donde $\gamma=\Gamma /\left(2 \pi R_{0} U_{\infty}\right)=(\pi / 4) /\left(c_{d} / \Delta\right)$, con $c_{d}$ y $\Delta$ el coeficiente de descarga y la constante del atomizador definidos en $(2.12)$ y $(2.17)$, respectivamente, y $\tilde{\theta}_{s_{s t \pm}}$ es el valor estacionario de $\tilde{\theta}$ en las superficies exterior e interior de la lámina líquida.

Consideraremos que el potencial de velocidades y la presión del líquido en régimen estacionario admiten los siguientes desarrollos:

$$
\begin{gathered}
\varphi_{s t}=\varphi_{s t 0}+W^{-2} \varphi_{s t 1}+W^{-4} \varphi_{s t 2}+O\left(W^{-6}\right) \\
p_{s t}=p_{s t 0}+W^{-2} p_{s t 1}+W^{-4} p_{s t 2}+O\left(W^{-6}\right)
\end{gathered}
$$

Sustituimos los anteriores desarrollos en (4.8) - (4.11) y agrupamos términos del mismo orden. Tomando los términos de órdenes $O\left(W^{4}\right)$ y $O\left(W^{2}\right)$ de la ecuación (4.8), y considerando las condiciones de contorno cinemáticas dadas por (4.10), se obtienen las siguientes expresiones para los primeros términos del desarrollo del potencial de velocidades del líquido (el análisis completo de este problema se presenta en el Apéndice D):

$$
\begin{gathered}
\varphi_{s t 0}=A_{0}(r) \\
\varphi_{s t 1}=A_{1}(r)+B_{1}(r) \tilde{\theta}
\end{gathered}
$$

De las condiciones de contorno dinámicas (4.11) se obtiene el primer término del desarrollo de la presión del líquido:

$$
p_{s t 0}=0
$$

Agrupando los términos de orden $O(1)$ en las ecuaciones (4.9) y (4.10), y teniendo en cuenta los anteriores resultados, se obtiene:

$$
\begin{aligned}
& \frac{\mathrm{d} A_{0}}{\mathrm{~d} r}=r\left[\left(\frac{\mathrm{d} \theta_{c}}{\mathrm{~d} r}\right)^{2}+\frac{1}{r^{2}}\right]^{1 / 2} \\
& B_{1}=\frac{\mathrm{d} \theta_{c}}{\mathrm{~d} r} r\left[\left(\frac{\mathrm{d} \theta_{c}}{\mathrm{~d} r}\right)^{2}+\frac{1}{r^{2}}\right]^{-1 / 2}
\end{aligned}
$$

Tomando ahora los términos de orden $O\left(W^{-2}\right)$ de la ecuación (4.9), y teniendo en cuenta que $p_{s t 1}\left(\tilde{\theta}_{s_{s t}}\right)=-p_{s t 1}\left(-\tilde{\theta}_{s t}\right)$, se obtiene la expresión:

$$
\frac{\mathrm{d} A_{1}}{\mathrm{~d} r}=\frac{-\gamma^{2}}{2 r \sin ^{2} \theta_{c}}\left[\left(\frac{\mathrm{d} \theta_{c}}{\mathrm{~d} r}\right)^{2}+\frac{1}{r^{2}}\right]^{1 / 2}
$$

y el valor de $p_{s t 1}$ :

$$
p_{s t 1}=-r\left[r \frac{\mathrm{d}^{2} \theta_{c}}{\mathrm{~d} r^{2}}+2 \frac{\mathrm{d} \theta_{c}}{\mathrm{~d} r}+r^{2}\left(\frac{\mathrm{d} \theta_{c}}{\mathrm{~d} r}\right)^{3}\right]\left[1+r^{2}\left(\frac{\mathrm{d} \theta_{c}}{\mathrm{~d} r}\right)^{2}\right]^{-2} \tilde{\theta}
$$


Particularizando la expresión para las condiciones de contorno cinemáticas (4.10) en las superficies exterior e interior de la lámina líquida, y tomando los términos de orden $O\left(W^{-2}\right)$, se llega a la siguiente ecuación diferencial para $\tilde{\theta}_{s_{s t}}$ :

$$
\frac{\partial \tilde{\theta}_{s_{s t}}}{\partial r}+\tilde{\theta}_{s_{s t}} \frac{\mathrm{d}}{\mathrm{d} r}\left[r^{2}\left(1+r^{2}\left(\frac{\mathrm{d} \theta_{c}}{\mathrm{~d} r}\right)^{2}\right)^{-1 / 2} \sin \theta_{c}\right]
$$

cuya solución es:

$$
\tilde{\theta}_{s_{s t}}=\frac{c_{d}}{4 r^{2} \sin \theta_{c}}\left[1+r^{2}\left(\frac{\mathrm{d} \theta_{c}}{\mathrm{~d} r}\right)^{2}\right]^{1 / 2}
$$

Seleccionando ahora los términos de orden $O\left(W^{-2}\right)$ de la ecuación (4.11) para las condiciones de contorno dinámicas, y sustituyendo los resultados obtenidos anteriormente, se llega finalmente a la siguiente ecuación diferencial para $\theta_{c}$ :

$$
\begin{aligned}
r\left(r \sin \theta_{c}-\frac{c_{d}}{4}\right) \frac{\mathrm{d}^{2} \theta_{c}}{\mathrm{~d} r^{2}}+\left(3 r \sin \theta_{c}-\frac{c_{d}}{2}\right) & \frac{\mathrm{d} \theta_{c}}{\mathrm{~d} r}-r^{2} \cos \theta_{c}\left(\frac{\mathrm{d} \theta_{c}}{\mathrm{~d} r}\right)^{2} \\
& +r^{2}\left(2 r \sin \theta_{c}-\frac{c_{d}}{4}\right)\left(\frac{\mathrm{d} \theta_{c}}{\mathrm{~d} r}\right)^{3}-\cos \theta_{c}=0
\end{aligned}
$$

La ecuación (4.20) se puede resolver numéricamente a partir del ángulo $\theta_{c 0}$ que forma la lámina cónica a la salida del inyector. Este ángulo está determinado por la solución en el interior de la cámara y el principio de la lámina, y es función decreciente del coeficiente de descarga $c_{d}$ y depende también de los parámetros geométricos de la cámara.

En la figura 4.2 se representa la forma de la lámina líquida dada por la solución de la ecuación (4.20) para diferentes valores del ángulo de dicha lámina a la salida del inyector, donde se ha considerado la relación entre este ángulo y el coeficiente de descarga calculada por Taylor (1948) e incluida en la Sección 2.3. Se observa que inicialmente la forma de la lámina líquida se aproxima a la de un cono, y que según avanza va divergiendo debido al efecto de la tensión superficial. 


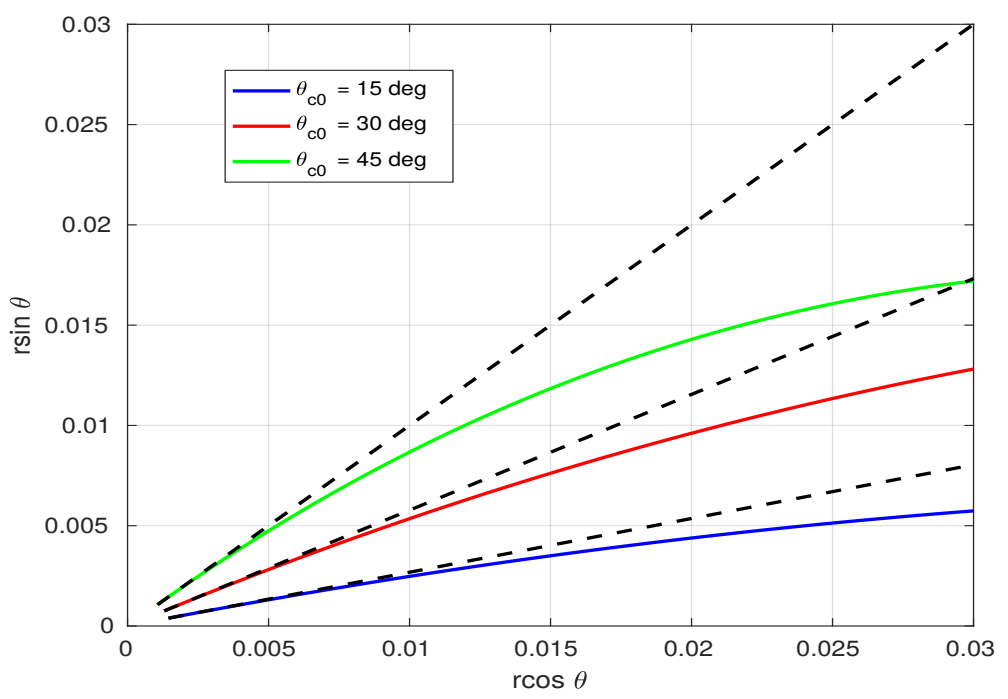

Figura 4.2 - Forma de la línea media de la lámina líquida para varios valores del ángulo de salida (en líneas discontínuas, posición de la lámina líquida si tuviera forma cónica).

\subsection{Estabilidad de la lámina líquida}

En ausencia de los efectos de la tensión superficial y del gas del ambiente, cada partícula fluida de la lámina viaja a velocidad constante, independientemente de la evolución de otras partículas fluidas. En estas condiciones, una perturbación que represente un pequeño cambio de altura de valor $\eta$ sobre la posición no perturbada de la lámina es transportada sin cambio de forma a la velocidad $U_{\infty}$ del líquido. El tiempo característico de paso de esta perturbación por un punto dado es $t_{1}=\lambda / U_{\infty}$, donde $\lambda$ es la longitud de onda característica de la perturbación. El paso de esta perturbación origina velocidades de orden $v_{g}=\eta / t_{1}$ en el gas, como consecuencia de la condición de contorno linealizada $\partial h / \partial t=v_{g}$, con $h \sim \eta \mathrm{y} t \sim t_{1}$. Estas velocidades llevan asociadas variaciones de presión de orden $p_{g}=\rho_{g} \lambda v_{g} / t_{1}=\rho_{g} U_{\infty}^{2} \eta / \lambda$, como consecuencia de la ecuación de Euler-Bernoulli linealizada en el gas $\rho_{g} \partial \varphi_{g} / \partial t+p_{g}=0$, donde $\rho_{g} \mathrm{y} \varphi_{g}$ son la densidad y el potencial de velocidades del gas, con $\varphi_{g} \sim v_{g} \lambda$.

En un sistema de referencia que se mueva con la velocidad $U_{\infty}$ del líquido no perturbado, estas variaciones de presión dan lugar a una aceleración del líquido normal a la lámina que hace crecer las perturbaciones, porque $p_{g}<0$ donde $\eta>0$, y viceversa. El 
tiempo característico del crecimiento de las perturbaciones es $t_{2}$, tal que:

$$
\rho h \frac{\eta}{t_{2}^{2}} \sim p_{g} \quad ; \quad t_{2} \sim \frac{h / U_{\infty}}{\alpha^{1 / 2}}\left(\frac{\lambda}{h}\right)^{1 / 2}
$$

donde $\alpha$ es la relación entre las densidades del gas y del líquido, $\alpha=\rho_{g} / \rho$. Este crecimiento es tanto más rápido cuanto menor sea $\lambda$. Sin embargo, la tensión superficial origina una fuerza $\sigma \partial^{2} h / \partial r^{2} \sim \sigma \eta / \lambda^{2}$ que se opone al crecimiento de las perturbaciones. Esta fuerza es importante cuando $\sigma \eta / \lambda^{2} \sim p_{g}$, es decir, para $\lambda \sim \lambda_{c}=\sigma /\left(\rho_{g} U_{\infty}^{2}\right)=h /(\alpha W e)$, con $W e=\rho U_{\infty}^{2} h / \sigma$, y se hace dominante cuando $\lambda \ll \lambda_{c}$. Para estas perturbaciones de longitud de onda $\lambda_{c}$ se tiene $t_{2} \sim\left(h / U_{\infty}\right) /\left(\alpha W e^{1 / 2}\right)$ y $t_{2} / t_{1} \sim W e^{1 / 2}$. La distancia $L$ a la que crecen las perturbaciones es:

$$
L=U_{\infty} t_{2} \sim \frac{R_{0}^{3 / 2} / L}{\alpha W^{1 / 2} / L^{1 / 2}}
$$

donde se ha usado la condición $h \sim R_{0}^{2} / L$. De esta forma, $L \sim R_{0} /\left(\alpha^{2 / 3} W^{1 / 3}\right), h \sim$ $R_{0} \alpha^{2 / 3} W^{1 / 3}, W e \sim\left(\alpha W^{2}\right)^{2 / 3}, \lambda_{c} \sim R_{0} /(\alpha W)$ y $L / r_{c} \sim\left(\alpha W^{2}\right)^{-2 / 3}$, donde $r_{c} \sim W R_{0}$.

Así pues, las perturbaciones crecen antes de alcanzar el límite de la lámina no perturbada $\left(r \sim r_{c}\right)$ cuando $\alpha W^{2} \gg 1$, en una región donde $W e$ es aún grande. Definimos el parámetro $\beta$ como:

$$
\beta=\alpha W^{2} \gg 1
$$

Si las inestabilidades se producen en la región en que $r \ll W R_{0}$, la lámina líquida sin perturbar se puede considerar cónica, luego $\mathrm{d} \theta_{c} / \mathrm{d} r=0$. Introducimos pequeñas perturbaciones a los potenciales de velocidades del líquido y del gas, $\varphi$ y $\varphi_{g}$, a las distribuciones de presión en el líquido y en el gas, $p$ y $p_{g}$, y a la posición de las superficies libres exterior e interior de la lámina líquida, $\tilde{\theta}_{s_{ \pm}}$, de forma que escribimos:

$$
\begin{gathered}
\varphi(r, \tilde{\theta})=\varphi_{s t}(r, \tilde{\theta})+\varphi_{p}(r, \tilde{\theta}, t) \\
p(r, \tilde{\theta})=p_{s t}(r, \tilde{\theta})+p_{p}(r, \tilde{\theta}, t) \\
\tilde{\theta}_{s_{ \pm}}(r)=\tilde{\theta}_{s_{s t \pm}}(r)+\tilde{\theta}_{s_{p \pm}}(r, t) \\
\varphi_{g}(r, \tilde{\theta})=\varphi_{g_{s t}}(r, \tilde{\theta})+\varphi_{g_{p}}(r, \tilde{\theta}, t) \\
p_{g}(r, \tilde{\theta})=p_{g_{s t}}(r, \tilde{\theta})+p_{g_{p}}(r, \tilde{\theta}, t)
\end{gathered}
$$

donde $\left(\varphi_{s t}, p_{s t}, \tilde{\theta}_{s_{s t \pm}}, \varphi_{g_{s t}}, p_{g_{s t}}\right)$ es la solución estacionaria, y $\left(\varphi_{p}, p_{p}, \tilde{\theta}_{s_{p \pm}}, \varphi_{g_{p}}, p_{g_{p}}\right)$ representan las perturbaciones. El problema en la región del líquido está gobernado por las 
siguientes ecuaciones linealizadas, en variables adimensionales, con $r$ escalada con $W R_{0}$, el potencial de velocidades con $W U_{\infty} R_{0}, p-p_{a}$ con $\rho U_{\infty}^{2}$, y el tiempo con $W R_{0} / U_{\infty}$ :

$$
\begin{gathered}
W^{4} \frac{1}{r^{2}} \frac{\partial^{2} \varphi_{p}}{\partial \tilde{\theta}^{2}}+W^{2} \frac{\cot \theta_{c}}{r^{2}} \frac{\partial \varphi_{p}}{\partial \tilde{\theta}}+\frac{1}{r^{2}} \frac{\partial}{\partial r}\left(r^{2} \frac{\partial \varphi_{p}}{\partial r}\right)-\frac{\tilde{\theta}}{r^{2} \sin ^{2} \theta_{c}} \frac{\partial \varphi_{p}}{\partial \tilde{\theta}}+O\left(W^{-2}\right)=0 \\
W^{4} \frac{1}{r^{2}} \frac{\partial \varphi_{s t}}{\partial \tilde{\theta}} \frac{\partial \varphi_{p}}{\partial \tilde{\theta}}+\left(\frac{\partial \varphi_{p}}{\partial t}+\frac{\partial \varphi_{s t}}{\partial r} \frac{\partial \varphi_{p}}{\partial r}+p_{p}\right)+O\left(W^{-4}\right)=0 \\
W^{2} \frac{1}{r^{2}}\left(\frac{\partial^{2} \varphi_{s t}}{\partial \tilde{\theta}^{2}} \tilde{\theta}_{s_{p \pm}}+\frac{\partial \varphi_{p}}{\partial \tilde{\theta}}\right)-W^{-2}\left[\frac{\partial \tilde{\theta}_{s_{p \pm}}}{\partial t}+\frac{\partial \tilde{\theta}_{s_{p \pm}}}{\partial r} \frac{\partial \varphi_{s t}}{\partial r}+\frac{\partial \tilde{\theta}_{s_{s t \pm}}}{\partial r}\left(\frac{\partial^{2} \varphi_{s t}}{\partial r \partial \tilde{\theta}} \tilde{\theta}_{s_{p \pm}}+\frac{\partial \varphi_{p}}{\partial r}\right)\right] \\
p_{p}+\frac{\partial p_{s t}}{\partial \tilde{\theta}} \tilde{\theta}_{s_{p \pm}}-p_{g_{p}} \pm W^{-4}\left[\frac { 1 } { r ^ { 2 } } \frac { \partial } { \partial r } \left(\begin{array}{c}
\left.\left.r^{3} \frac{\partial \tilde{\theta}_{s_{p \pm}}}{\partial r}\right)+\frac{\tilde{\theta}_{s_{p \pm}}}{r \sin ^{2} \theta_{c}}\right] \\
+O\left(W^{-6}\right)=0 \quad \text { en } \quad \theta=W_{c}+\tilde{\theta}_{s_{ \pm}}
\end{array} \quad(4.25)\right.\right. \\
\theta^{-2} \tilde{\theta}_{s_{ \pm}}
\end{gathered}
$$

donde se ha tenido en cuenta que $\mathrm{d} \theta_{c} / \mathrm{d} r=0$. Consideraremos que las perturbaciones en el potencial de velocidades, en la presión y en la posición de la superficie libre de la lámina líquida admiten los siguientes desarrollos:

$$
\varphi_{p}=W^{-4} \varphi_{p 0}+\ldots \quad ; \quad p_{p}=W^{-4} p_{p 0}+\ldots \quad ; \quad \tilde{\theta}_{s_{p \pm}}=\tilde{\theta}_{s_{p 0 \pm}}+\ldots
$$

Las perturbaciones se pueden descomponer en modos simétrico, en el que $\tilde{\theta}_{s_{p+}}=-\tilde{\theta}_{s_{p-}}$, y antisimétrico, para el cual $\tilde{\theta}_{s_{p+}}=\tilde{\theta}_{s_{p-}}$. De estos modos, el que presenta un mayor grado de inestabilidad es el antisimétrico (Squire, 1953), que es el que se estudiará a continuación.

El análisis completo de este problema se presenta en el Apéndice E. Tomando los términos de orden $O(1)$ en la ecuación (4.23) se obtiene:

$$
\varphi_{p 0}=A_{p 0}(r, t)+B_{p 0}(r, t) \tilde{\theta}
$$

Agrupando los términos de orden $O\left(W^{-2}\right)$ en la ecuación (4.25) y considerando perturbaciones antisimétricas:

$$
B_{p 0}=\frac{\partial\left(r^{2} \tilde{\theta}_{s_{p 0}}\right)}{\partial t}+\frac{\partial\left(r^{2} \tilde{\theta}_{s_{p 0}}\right)}{\partial r}
$$

Por otro lado, tomando los términos de orden $O\left(W^{-4}\right)$ en la expresión (4.24) y particularizando en las superficies exterior e interior de la lámina líquida se obtiene la siguiente relación para $p_{p 0}$ :

$$
p_{p 0}=-\tilde{\theta}_{s_{s t}}\left(\frac{\partial}{\partial t}+\frac{\partial}{\partial r}-\frac{2}{r}\right)\left(\frac{\partial}{\partial t}+\frac{\partial}{\partial r}\right)\left(r^{2} \tilde{\theta}_{s_{p 0}}\right)
$$


Agrupando ahora los términos de orden $O\left(W^{-4}\right)$ de la ecuación (4.26):

$$
p_{p 0}+\frac{\partial p_{s t 2}}{\partial \tilde{\theta}} \tilde{\theta}_{s_{p 0}}-p_{g_{p}}+\frac{1}{r^{2}} \frac{\partial}{\partial r}\left(r^{3} \frac{\partial \tilde{\theta}_{s_{p 0}}}{\partial r}\right)+\frac{\tilde{\theta}_{s_{p 0}}}{r \sin ^{2} \theta_{c}}=0 \quad \text { en } \quad \tilde{\theta}=\tilde{\theta}_{s_{s t \pm}}
$$

donde $p_{s t 2}$ se obtiene de agrupar los términos de orden $O\left(W^{-4}\right)$ en la ecuación (4.9):

$$
p_{s t 2}=-\tilde{\theta}^{2}-\frac{B_{2}^{2}}{2 r^{2}}-\frac{\mathrm{d} A_{2}}{\mathrm{~d} r}-\frac{\gamma^{4}}{8 r^{4} \sin ^{4} \theta_{c}}
$$

Introduciendo (4.30) y (4.32) en (4.31):

$$
\begin{aligned}
-\frac{c_{d}}{4 r^{2} \sin \theta_{c}}( & \left.\frac{\partial}{\partial t}+\frac{\partial}{\partial r}-\frac{2}{r}\right)\left(\frac{\partial}{\partial t}+\frac{\partial}{\partial r}\right)\left(r^{2} \tilde{\theta}_{s_{p 0}}\right) \\
& -\frac{c_{d}}{2 r^{2} \sin \theta_{c}} \tilde{\theta}_{s_{p 0}}-p_{g_{p}}\left(\tilde{\theta}=\tilde{\theta}_{s_{s t}}\right)+\frac{1}{r^{2}} \frac{\partial}{\partial r}\left(r^{3} \frac{\partial \tilde{\theta}_{s_{p 0}}}{\partial r}\right)+\frac{\tilde{\theta}_{s_{p 0}}}{r \sin ^{2} \theta_{c}}=0
\end{aligned}
$$

La longitud de onda de las perturbaciones es larga en comparación con el espesor de la lámina, pero corta en comparación con la extensión de la misma, pues como vimos anteriormente $\lambda_{c} / h \sim \alpha^{-1} \beta^{-2 / 3}$ y $\lambda_{c} / r_{c} \sim \beta^{-1}$. Como estamos considerando perturbaciones de pequeña amplitud, y su variación es lenta con respecto a $r$, podemos buscar soluciones de este problema con el método WKB. Para ello introducimos una nueva función desconocida, $s(r)$, y una nueva constante, $A$, y expresamos $\tilde{\theta}_{s_{p 0}}$ en términos de ellas mediante la relación:

$$
\tilde{\theta}_{s_{p 0}}=A e^{i s(r)-i \omega t}
$$

donde forzamos con la frecuencia $\omega$ constante.

Resta por determinar la perturbación en la presión del gas. Las ecuaciones linealizadas que gobiernan el problema son las siguientes:

$$
\begin{gathered}
\frac{1}{r^{2}} \frac{\partial}{\partial r}\left(r^{2} \frac{\partial \varphi_{g_{p}}}{\partial r}\right)+\frac{1}{r^{2} \sin \theta} \frac{\partial}{\partial \theta}\left(\sin \theta \frac{\partial \varphi_{g_{p}}}{\partial \theta}\right)=0 \\
\alpha \frac{\partial \varphi_{g_{p}}}{\partial t}+p_{g_{p}}=0 \\
\frac{\partial \tilde{\theta}_{s_{p \pm}}}{\partial t}+\frac{\partial \tilde{\theta}_{s_{s t \pm}}}{\partial r} \frac{\partial \varphi_{g_{p}}}{\partial r}=\frac{W^{2}}{r^{2}} \frac{\partial \varphi_{g_{p}}}{\partial \theta} \quad \text { en } \quad \theta=\theta_{c}+W^{-2} \tilde{\theta}_{s_{s t \pm}}
\end{gathered}
$$

donde $\alpha=\rho_{g} / \rho$, siendo $\rho_{g}$ la densidad del gas. Introducimos los siguientes desarrollos para las perturbaciones en el potencial de velocidades y en la presión en el gas:

$$
\varphi_{g_{p}}=W^{-2} \varphi_{g_{p 0}}+\ldots \quad ; \quad p_{g_{p}}=W^{-4} p_{g_{p 0}}+\ldots
$$


Debido a que estamos considerando perturbaciones de pequeña amplitud, y su variación es lenta en el espacio, podemos aplicar también el método WKB al problema del gas, para lo que introducimos la nueva función desconocida $f(r, \theta)$ y expresamos $\varphi_{g_{p 0}}$ en términos de ella como sigue:

$$
\varphi_{g_{p 0}}=f(r, \theta) e^{i s(r)-i \omega t}
$$

Las funciones $s(r)$ y $f(r, \theta)$ admiten desarrollos de la forma $s(r)=s_{0}(r)+s_{1}(r)+\ldots$, con $s_{0}=O(\beta)$ y $s_{1}=O(1)$, y $f(r, \theta)=f_{0}(r, \theta)+f_{1}(r, \theta)+\ldots$, con $f_{0}=O(1)$ y $f_{1}=O\left(\beta^{-1}\right)$. Insertando (4.38) en (4.35) - (4.37) y agrupando términos del mismo orden se obtiene:

$$
\begin{gathered}
f_{0}=B_{g 0} e^{-r \frac{\mathrm{d} s_{0}}{\mathrm{~d} r}\left(\theta-\theta_{c}\right)} \\
f_{1}=\left[A_{g 1}+B_{g 1}\left(\theta-\theta_{c}\right)+C_{g 1}\left(\theta-\theta_{c}\right)^{2}\right] e^{-r \frac{\mathrm{d} s_{0}}{\mathrm{~d} r}\left(\theta-\theta_{c}\right)}
\end{gathered}
$$

con:

$$
\begin{gathered}
B_{g 0}=i \omega r\left(\frac{\mathrm{d} s_{0}}{\mathrm{~d} r}\right)^{-1} A \\
A_{g 1}=i \omega\left(\frac{\mathrm{d} s_{0}}{\mathrm{~d} r}\right)^{-2}\left(\frac{i}{2}-r \frac{\mathrm{d} s_{1}}{\mathrm{~d} r}-\frac{\cot \theta_{c}}{2}\right) A \\
B_{g 1}=\left(\frac{i}{2}-r \frac{\mathrm{d} s_{1}}{\mathrm{~d} r}-\frac{\cot \theta_{c}}{2}\right) B_{g 0} \\
C_{g 1}=-i \frac{r}{2} \frac{\mathrm{d}}{\mathrm{d} r}\left(r \frac{\mathrm{d} s_{0}}{\mathrm{~d} r}\right) B_{g 0}
\end{gathered}
$$

Particularizando la ecuación (4.36) en $\theta=\theta_{c}$

$$
p_{g_{p 0}}=i \beta \omega\left(B_{g 0}+A_{g 1}\right) e^{i s-i \omega t}
$$

Llevando los valores de $B_{g 0}$ y $A_{g 1}$ dados por (4.41) y (4.42) a (4.45) se obtiene finalmente la expresión para la perturbación de la presión en el gas:

$$
p_{g_{p 0}}=i \beta \omega\left[i r \omega\left(\frac{\mathrm{d} s_{0}}{\mathrm{~d} r}\right)^{-1}+i \omega\left(\frac{\mathrm{d} s_{0}}{\mathrm{~d} r}\right)^{-2}\left(\frac{i}{2}-r \frac{\mathrm{d} s_{1}}{\mathrm{~d} r}-\frac{\cot \theta_{c}}{2}\right)\right] A e^{i s-i \omega t}
$$

Introduciendo (4.34) y (4.46) en (4.33), desarrollando y agrupando términos del mismo orden:

$$
\begin{gathered}
\frac{c_{d}}{4 \sin \theta_{c}}\left(-\omega+\frac{\mathrm{d} s_{0}}{\mathrm{~d} r}\right)^{2}-r\left(\frac{\mathrm{d} s_{0}}{\mathrm{~d} r}\right)^{2}+\beta r \omega^{2}\left(\frac{\mathrm{d} s_{0}}{\mathrm{~d} r}\right)^{-1}=0 \\
\frac{c_{d}}{4 \sin \theta_{c}}\left[2\left(-\omega+\frac{\mathrm{d} s_{0}}{\mathrm{~d} r}\right)\left(\frac{\mathrm{d} s_{1}}{\mathrm{~d} r}-\frac{i}{r}\right)-i \frac{\mathrm{d}^{2} s_{0}}{\mathrm{~d} r^{2}}\right]-2 r \frac{\mathrm{d} s_{0}}{\mathrm{~d} r} \frac{\mathrm{d} s_{1}}{\mathrm{~d} r} \\
+3 i \frac{\mathrm{d} s_{0}}{\mathrm{~d} r}+i r \frac{\mathrm{d}^{2} s_{0}}{\mathrm{~d} r^{2}}+\beta \omega^{2}\left(\frac{\mathrm{d} s_{0}}{\mathrm{~d} r}\right)^{-2}\left(\frac{i}{2}-r \frac{\mathrm{d} s_{1}}{\mathrm{~d} r}-\frac{\cot \theta_{c}}{2}\right)=0
\end{gathered}
$$


De (4.47) se obtiene finalmente la siguiente relación de dispersión:

$$
\left(-\Omega+S^{\prime}\right)^{2} S^{\prime}+X\left(\Omega^{2}-S^{\prime 3}\right)=0
$$

donde:

$$
X=\frac{4 r \sin \theta_{c}}{c_{d}} \quad ; \quad \Omega=\frac{\omega}{\beta} \quad ; \quad S^{\prime}=\frac{1}{\beta} \frac{\mathrm{d} s_{0}}{\mathrm{~d} r}
$$

La expresión (4.49) se puede escribir como:

$$
(1-X) S^{\prime 3}-2 \Omega S^{\prime 2}+\Omega^{2} S^{\prime}+X \Omega^{2}=0
$$

El estudio de las raíces de la ecuación (4.51), que relaciona $S^{\prime}$ con $X$ para un valor de $\Omega$ dado permite analizar la estabilidad de la lámina líquida. La lámina es inestable en el caso en el que se presenten raíces complejas con parte imaginaria negativa.

1. Si $X<1$, las raíces de (4.51) pueden ser:

- Una raíz real negativa y dos raíces complejas conjugadas con parte real positiva.

- Tres raíces reales, una negativa y dos positivas.

2. Si $X>1$, las raíces de (4.51) pueden ser:

- Una raíz real positiva y dos raíces complejas conjugadas con parte real negativa.

- Tres raíces reales, una positiva y dos negativas.

Debido a que estamos estudiando la evolución de las perturbaciones en la zona de la lámina próxima a la salida del atomizador, el caso que nos interesa es el de $X<1$. La presencia de raíces complejas en este caso, que es la situación en la que la lámina es inestable, se da para $\Omega<1$, siempre y cuando $X$ sea menor que un cierto valor $X_{c}(\Omega)$ decreciente con $\Omega$. En la figuras 4.3(a) y 4.3(b) se muestran, respectivamente, la parte imaginaria $S_{i m}^{\prime}$ y la parte real $S_{r e}^{\prime}$ de las raíces complejas conjugadas de (4.51) en función de $X$, para varios valores de $\Omega$. El valor de $X_{c}$ se corresponde con el corte con el eje de abscisas de las curvas de $S_{i m}^{\prime}$ representadas. Se observa que al aumentar el valor de $\Omega$ disminuye el de $X_{c}$. En las figuras $4.3(\mathrm{c})$ y $4.3(\mathrm{~d})$ se muestra la parte imginaria de $S=\left(4 \sin \theta_{c}\right) s_{0} /\left(\beta c_{d}\right)$ en función de $X$ y de $\Omega$, con el valor máximo de la misma en función de $\Omega$. 

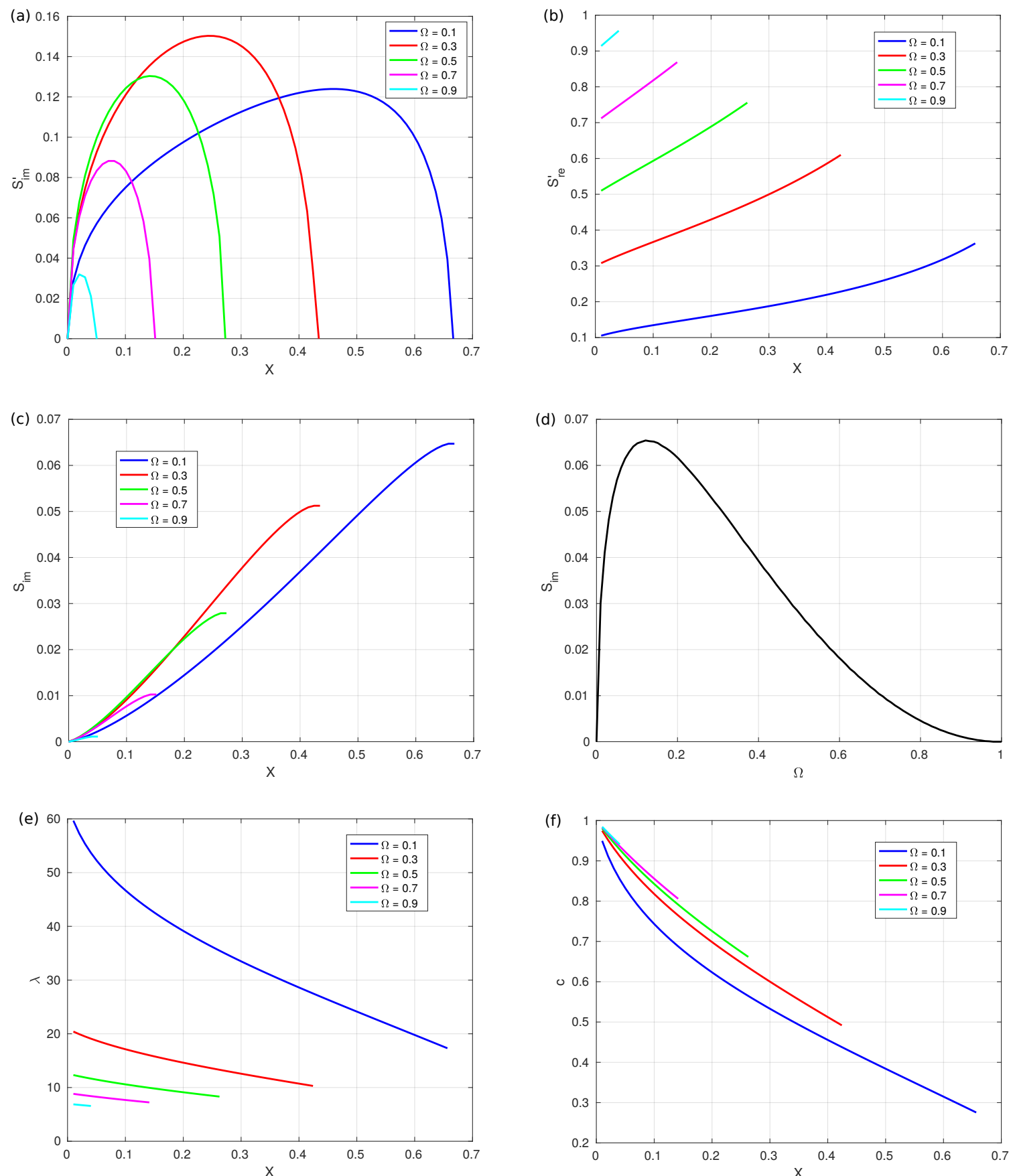

Figura 4.3 - (a) Parte imaginaria de $S^{\prime}$ en función de $X$ y de $\Omega$. (b) parte real de $S^{\prime}$ en función de $X$ y de $\Omega$. (c) Parte imaginaria de $S$ en función de $X$ y de $\Omega$. (d) Valor máximo de la parte imaginaria de $S$ en función de $\Omega$. (e) Longitud de onda $\lambda$ en función de $X$ y de $\Omega$. (f) Velocidad de propagación de las ondas $c$ respecto al atomizador en función de $X$ y de $\Omega$. 
En ausencia del gas del ambiente, esto es, haciendo $\beta=0$ en la relación de dispersión dada por la ecuación (4.51):

$$
(1-X)\left(\frac{\mathrm{d} s_{0}}{\mathrm{~d} r}\right)^{2}-2 \omega \frac{\mathrm{d} s_{0}}{\mathrm{~d} r}+\omega^{2}=0
$$

que da lugar a:

$$
\frac{\mathrm{d} s_{0}}{\mathrm{~d} r}=\omega \frac{1 \pm \sqrt{X}}{1-X}
$$

Esto es, las ondas en la lámina son estables, pues los valores de $s_{0}$ son reales.

Llamamos $k=\left|S_{r e}^{\prime}\right|$, y la longitud de onda $\lambda=2 \pi / k$. Ésta se representa en la figura 4.3(e) en función de $X$ y de $\Omega$. Se observa que la longitud de onda disminuye según la onda avanza por la lámina líquida, y cuando aumenta la frecuencia con la que se fuerza. La figura 4.3(f) muestra que la velocidad de propagación de las ondas con respecto al atomizador, $c=\Omega / k$, disminuye con la distancia recorrida a lo largo de la lámina, $\mathrm{y}$ aumenta con $\Omega$. Sin embargo, la velocidad de propagación de las ondas con respecto al fluido, $1-c$, es nula a la salida del atomizador y aumenta según las ondas se alejan del mismo. Estos resultados están de acuerdo con los obtenidos experimentalmente por Bremond, Clanet \& Villermaux (2007).

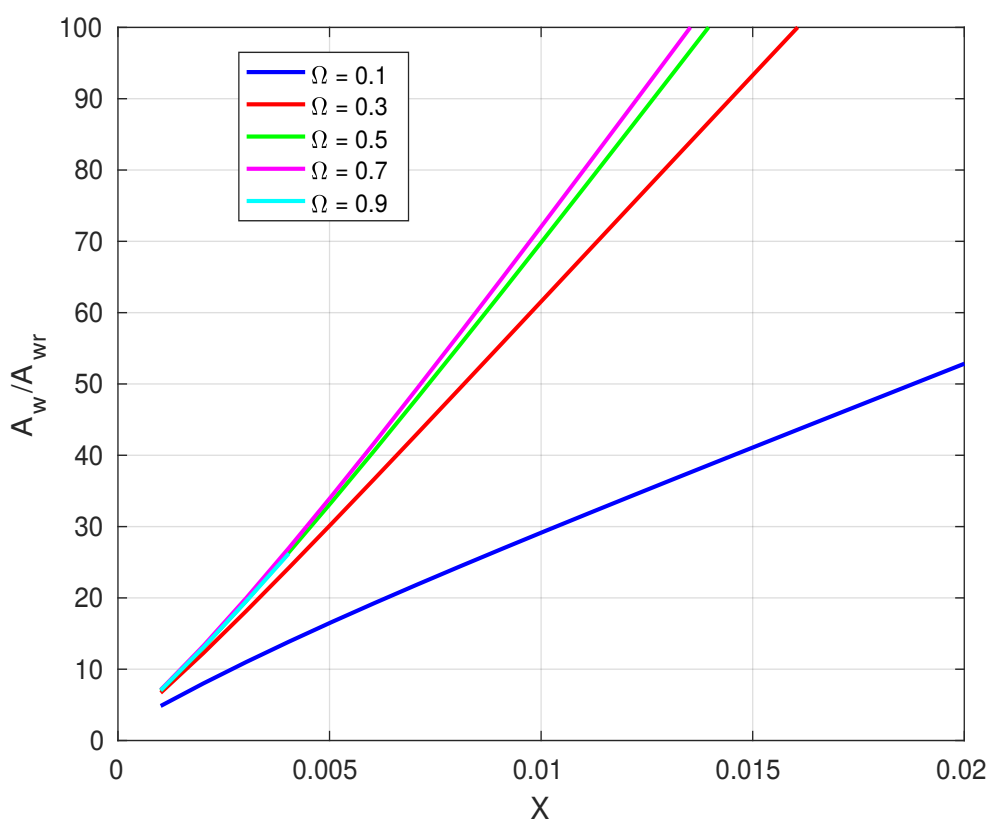

Figura 4.4 - Variación de la amplitud de las ondas con la distancia, para varios valores de la frecuencia $\left(\theta_{c}=45\right)$. 
Por otro lado, de la relación (4.48) se extrae:

$$
\begin{aligned}
& {\left[2 S^{\prime 2}\left(-\Omega+S^{\prime}\right)-X\left(\Omega^{2}+2 S^{\prime 3}\right)\right] \frac{\mathrm{d} s_{1}}{\mathrm{~d} X}} \\
& \quad=2 i \frac{S^{\prime 2}}{X}\left(-\Omega+S^{\prime}\right)+\frac{i}{3}(1-X) \frac{\mathrm{d} S^{\prime 3}}{\mathrm{~d} X}-3 i S^{\prime 3}-\frac{i-\cot \theta_{c}}{2} \Omega
\end{aligned}
$$

La amplitud de las ondas es $A_{w}=A e^{s_{1 i m}}$, donde $s_{1 i m}$ es la parte imaginaria de $s_{1}$. Teniendo en cuenta (4.53), se obtiene la siguiente expresión para la variación de dicha amplitud:

$$
\frac{A_{w}}{A_{w r}}=\exp \left[\operatorname{Im}\left(\int_{X_{r}}^{X} \frac{2 i \frac{S^{\prime 2}}{X}\left(-\Omega+S^{\prime}\right)+\frac{i}{3}(1-X) \frac{\mathrm{d} S^{\prime 3}}{\mathrm{~d} X}-3 i S^{\prime 3}-\frac{i-\cot \theta_{c}}{2} \Omega}{2 S^{\prime 2}\left(-\Omega+S^{\prime}\right)-X\left(\Omega^{2}+2 S^{\prime 3}\right)} \mathrm{d} X\right)\right]
$$

donde $I m$ indica parte imaginaria, y $A_{w r}$ es el valor de la amplitud a una cierta distancia $X=X_{r}$. La variación de la amplitud se muestra en la figura 4.4, para varios valores de $\Omega$, pudiéndose observar que crece con la distancia al atomizador.

\subsection{Inestabilidad de Rayleigh-Taylor}

Si la amplitud de las ondas y su velocidad relativa al líquido superan ciertos valores, la inestabilidad de Kelvin-Helmholtz estudiada antes es capaz de dar lugar a una estabilidad secundaria de Rayleigh-Taylor. Taylor (1950b) mostró que, cuando dos fluidos de diferentes densidades superpuestos son acelerados con una aceleración normal a su entrefase, dicha entrefase será inestable si la aceleración es dirigida hacia el fluido de mayor densidad. Keller \& Kolodner (1954) extendieron el estudio de Taylor (1950b) al problema de la inestabilidad de una lámina líquida que separa dos gases, teniendo en cuenta el efecto de la tensión superficial.

Keller \& Kolodner (1954) consideraron una lámina líquida bidimensional de espesor $h$, densidad $\rho$ y tensión superficial $\sigma$ sometida a una aceleración constante $a$ normal a la misma. Expresando las perturbaciones en las superficies de la lámina en modos de la forma $\exp \left(i k_{T} x-i \omega_{T} t\right)$ obtienen la siguiente relación de dispersión, en las variables adimensionales usadas en este capítulo:

$$
\omega_{T}^{2}=W^{-2} k_{T}^{3} \operatorname{coth}\left(k_{T} h\right)\left[1 \pm \sqrt{1-\left(1-W^{4} \frac{a^{2}}{k_{T}^{4}}\right) \tanh ^{2}\left(k_{T} h\right)}\right]
$$

que, para el caso en que $k_{T} h \ll 1$, $\left(\tanh \left(k_{T} h\right) \approx k_{T} h\right)$ se reduce a:

$$
\omega_{T}^{2} \approx W^{-2} \frac{k_{T}^{4} h}{2}\left(1-W^{4} \frac{a^{2}}{k_{T}^{4}}\right)
$$


Si el paréntesis en la expresión (4.55) es negativo, entonces se obtienen valores imaginarios de $\omega_{T}$ que dan lugar a un crecimiento exponencial de la perturbación. Así pues, las ondas serán inestables si:

$$
1-W^{4} \frac{a^{2}}{k_{T}^{4}}<0
$$

de donde se obtiene un número de onda crítico $k_{c}$, tal que la inestabilidad se produce si $0<k_{T}<k_{c}$ :

$$
k_{c}=W \sqrt{a}
$$

El máximo ritmo de crecimiento $\omega_{T \max }$ se extrae del valor máximo de $\omega_{T}$ de la expresión (4.56) para números de onda menores que $k_{c}$ :

$$
\omega_{T_{\max }}=W a \sqrt{\frac{h}{2}}
$$

Admitiremos que estos resultados son aplicables a la lámina cónica estudiada en este capítulo, empleando valores locales del espesor y la aceleración.

La inestabilidad de Rayleigh-Taylor induce variaciones de espesor en la lámina líquida, particularmente en la dirección azimutal, que causan la formación de ligamentos longitudinales que posteriormente se rompen en gotas. Si la inestabilidad tiene tiempo de desarrollarse, estas variaciones de espesor se amplificarán. Esto sucede si el tiempo de residencia de las partículas fluidas en la región de máxima aceleración, en torno a una cresta de una onda de Kelvin-Helmholtz, $\tau_{c}$, es del orden del tiempo característico de crecimiento de la inestabilidad de Rayleigh-Taylor, $\omega_{T_{\max }}^{-1}$, siendo $\omega_{T_{\max }}$ el máximo crecimiento de la inestabilidad de Rayleigh-Taylor. Así pues, el criterio para que esta inestabilidad se desarrolle es:

$$
\tau_{c}=\omega_{T_{\max }}^{-1}
$$

Para poder usar este criterio es preciso calcular separadamente $\omega_{T_{\text {max }}}^{-1} \mathrm{y} \tau_{c}$.

\subsubsection{Máximo ritmo de crecimiento de la inestabilidad de Rayleigh- Taylor}

La aceleración del fluido en la lámina no es constante. Su valor a, en variables adimensionales, es:

$$
\mathbf{a}=\frac{\partial \mathbf{u}_{\mathbf{p}}}{\partial t}+\mathbf{e}_{\mathbf{r}} \cdot \nabla \mathbf{u}_{\mathbf{p}}
$$

donde $\mathbf{u}_{\mathbf{p}}$ representa las perturbaciones en el vector velocidad y $\mathbf{e}_{\mathbf{r}}$ es el vector unitario en la dirección de $r$. La componente de la aceleración en la dirección de $\theta, a_{\theta}$, normal a 
la lámina, teniendo en cuenta (4.27), es:

$$
a_{\theta}=\frac{W^{-2}}{r}\left(\frac{\partial^{2} \varphi_{p 0}}{\partial t \partial \tilde{\theta}}+\frac{\partial^{2} \varphi_{p 0}}{\partial r \partial \tilde{\theta}}\right)
$$

que, considerando (4.28), se reduce a:

$$
a_{\theta}=\frac{W^{-2}}{r}\left(\frac{\partial B_{p 0}}{\partial t}+\frac{\partial B_{p 0}}{\partial r}\right)
$$

Introduciendo (4.34) en (4.29):

$$
B_{p 0}=\left[i r^{2}\left(-\omega+\frac{\mathrm{d} s}{\mathrm{~d} r}\right)+2 r\right] A e^{i s-i \omega t}
$$

Sustituyendo (4.62) en (4.61) se obtiene la aceleración normal $a_{\theta}$ :

$$
a_{\theta}=W^{-2}\left[-r\left(-\omega+\frac{\mathrm{d} s}{\mathrm{~d} r}\right)^{2}+4 i\left(-\omega+\frac{\mathrm{d} s}{\mathrm{~d} r}\right)+i r \frac{\mathrm{d}^{2} s}{\mathrm{~d} r^{2}}+\frac{2}{r}\right] A e^{i s-i \omega t}
$$

cuyo valor aproximado es, tomando los términos de mayor orden:

$$
a_{\theta}=-W^{-2} r\left(-\omega+\frac{\mathrm{d} s_{0}}{\mathrm{~d} r}\right)^{2} A e^{i s-i \omega t}
$$

La parte real de la aceleración normal es:

$$
a_{\theta r e}=-W^{-2} r\left[\left(-\omega+\frac{\mathrm{d} s_{0 r e}}{\mathrm{~d} r}\right)^{2}-\left(\frac{\mathrm{d} s_{0 i m}}{\mathrm{~d} r}\right)^{2}\right] A_{w} e^{s_{0 i m}} \cos \left(s_{0 r e}-\omega t\right)
$$

y su valor máximo:

$$
a_{\theta_{\text {max }}}=W^{-2} r\left[\left(-\omega+\frac{\mathrm{d} s_{0 r e}}{\mathrm{~d} r}\right)^{2}-\left(\frac{\mathrm{d} s_{0 i m}}{\mathrm{~d} r}\right)^{2}\right] A_{w} e^{s_{0 i m}}
$$

donde $s_{0 r e} \mathrm{y} s_{0 i m}$ son la parte real y la parte imaginaria de $s_{0}$, respectivamente.

El espesor $h$ de la lámina líquida también varía a lo largo de la misma, y viene dado por (4.3) y (4.19):

$$
h=\frac{c_{d}}{2 r \sin \theta_{c}}
$$

Introduciendo la aceleración (4.66) y el espesor (4.67) en la expresión (4.58) del máximo ritmo de crecimiento de la inestabilidad de Rayleigh-Taylor se obtiene:

$$
\omega_{T_{\text {max }}}=W^{-1} \sqrt{\frac{r c_{d}}{4 \sin \theta_{c}}}\left[\left(-\omega+\frac{\mathrm{d} s_{0 r e}}{\mathrm{~d} r}\right)^{2}-\left(\frac{\mathrm{d} s_{0 i m}}{\mathrm{~d} r}\right)^{2}\right] A_{w} e^{s_{0 i m}}
$$


que expresado en función de las variables definidas en (4.50):

$$
\omega_{T_{\max }}=\beta^{2} W^{-1} \frac{c_{d}}{4 \sin \theta_{c}} \sqrt{X}\left[\left(-\Omega+S_{r e}^{\prime}\right)^{2}-S_{i m}^{\prime 2}\right] A_{w} e^{\beta S_{i m}}
$$

El valor del máximo ritmo de crecimiento de la inestabilidad de Rayleigh-Taylor aumenta con la distancia al atomizador, como se observa en la figura 4.5.

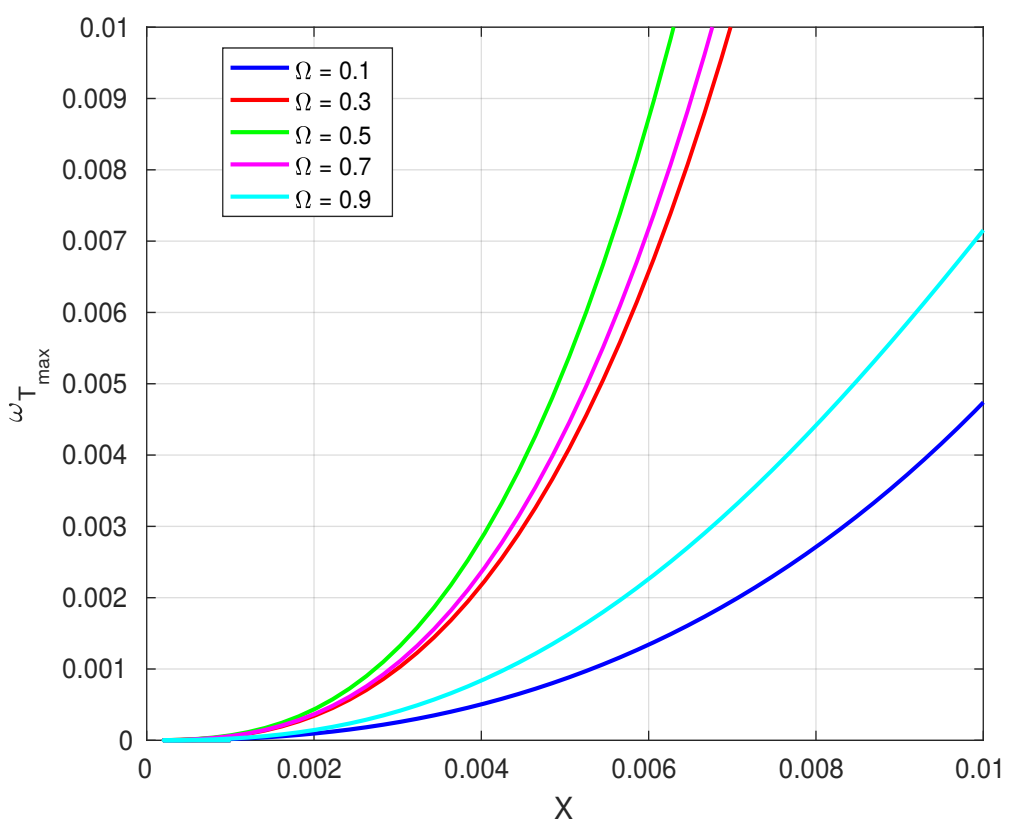

Figura 4.5 - Máximo ritmo de crecimiento de la inestabilidad de Rayleigh-Taylor en función de la distancia $X$, para varios valores de $\Omega\left(\operatorname{con} \beta^{2} W^{-1} A_{w r} c_{d} / 4 \sin \theta_{c}=1 \mathrm{y}\right.$ $\left.\beta=10^{3}\right)$.

\subsubsection{Tiempo de residencia en la región de máxima aceleración}

La distancia que recorre una partícula fluida en la región de máxima aceleración, en las inmediaciones de una cresta de la onda, es del orden de la longitud de onda, y la velocidad con que lo hace es la velocidad relativa entre el líquido y la onda. Para valores pequeños de $r$ la velocidad con que se desplazan las ondas es aproximada a la del líquido, luego la velocidad relativa de una partícula fluida a la onda es pequeña. Esta velocidad relativa irá aumentando con $r$, pues la velocidad de las ondas respecto al atomizador disminuye con la distancia. Así pues, el tiempo de tránsito de una partícula fluida por las inmediaciones de una cresta de la onda será del orden de la longitud de onda dividida por la velocidad 
relativa entre el líquido y las ondas, esto es:

$$
\tau_{c}=\frac{2 \pi /\left|S_{r e}^{\prime}\right|}{1-\Omega /\left|S_{r e}^{\prime}\right|}=\frac{2 \pi}{\left|S_{r e}^{\prime}\right|-\Omega}
$$

La expresión (4.70) es una función decreciente con la distancia al atomizador, (ver la figura 4.6), ya que la longitud de onda decrece con la distancia, mientras que la velocidad relativa entre el fluido y las ondas aumenta, como se muestra en las figuras 4.3(e) y 4.3(f), respectivamente.

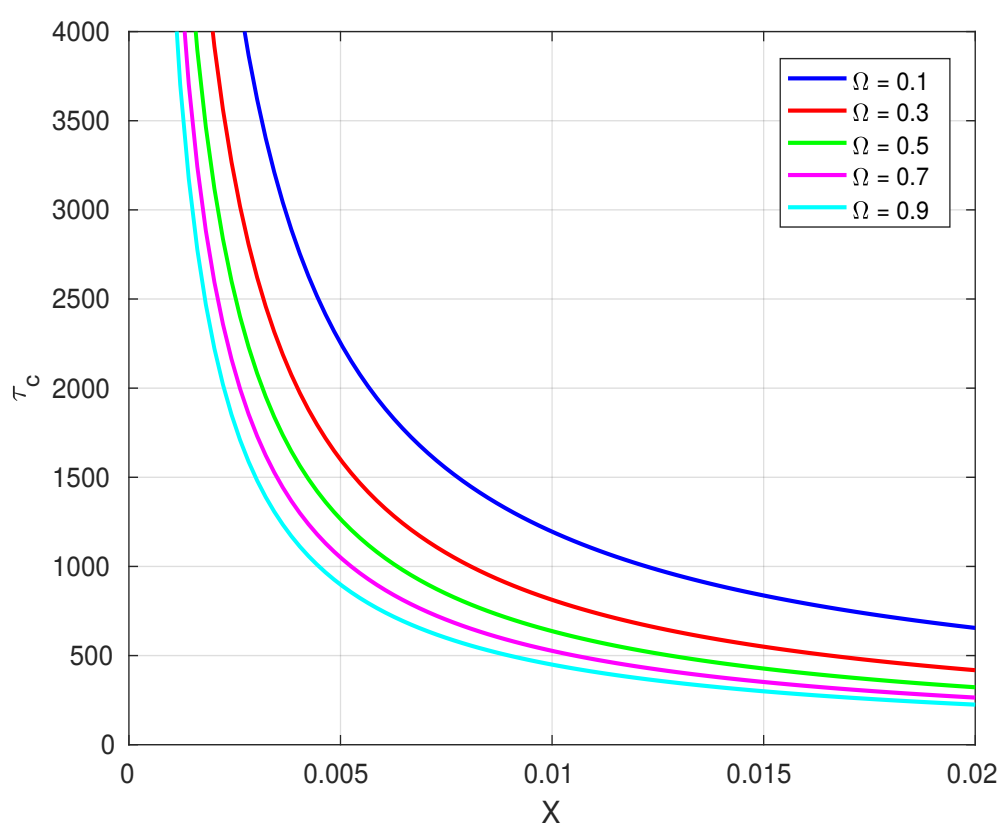

Figura 4.6 - Tiempo de tránsito de una partícula fluida en la región de máxima aceleración en función de la distancia $X$, para varios valores de $\Omega$.

\subsubsection{Criterio de inestabilidad}

Introduciendo (4.69) y (4.70) en (4.59) se obtiene finalmente una expresión en función de $X$ que determina la distancia a la que se produce la inestabilidad de Rayleigh-Taylor:

$$
\beta^{2} W^{-1} \frac{\pi c_{d}}{2 \sin \theta_{c}} \sqrt{X}\left[\left(-\Omega+S_{r e}^{\prime}\right)^{2}-S_{i m}^{\prime 2}\right] A_{w} e^{\beta S_{i m}}-\left|S_{r e}^{\prime}\right|+\Omega=0
$$

En la figura 4.7(a) se muestran las curvas de $\tau_{c}$ (líneas contínuas) y de $\omega_{T_{\max }}^{-1}$ (líneas discontinuas) en función de la distancia $X$, para varios valores de la frecuencia. El punto donde ambas curvas se cortan determina la distancia $X_{R T}$ al atomizador a la que se 
rompe la lámina por efecto de la inestabilidad de Rayleigh-Taylor. En la figura 4.7(b) se observa que dicha distancia disminuye al aumentar la frecuencia $\Omega$ hasta alcanzar un valor mínimo, a partir del cual aumenta con $\Omega$. También puede verse que $X_{R T}$ disminuye cuando aumenta el número de Weber $W$.
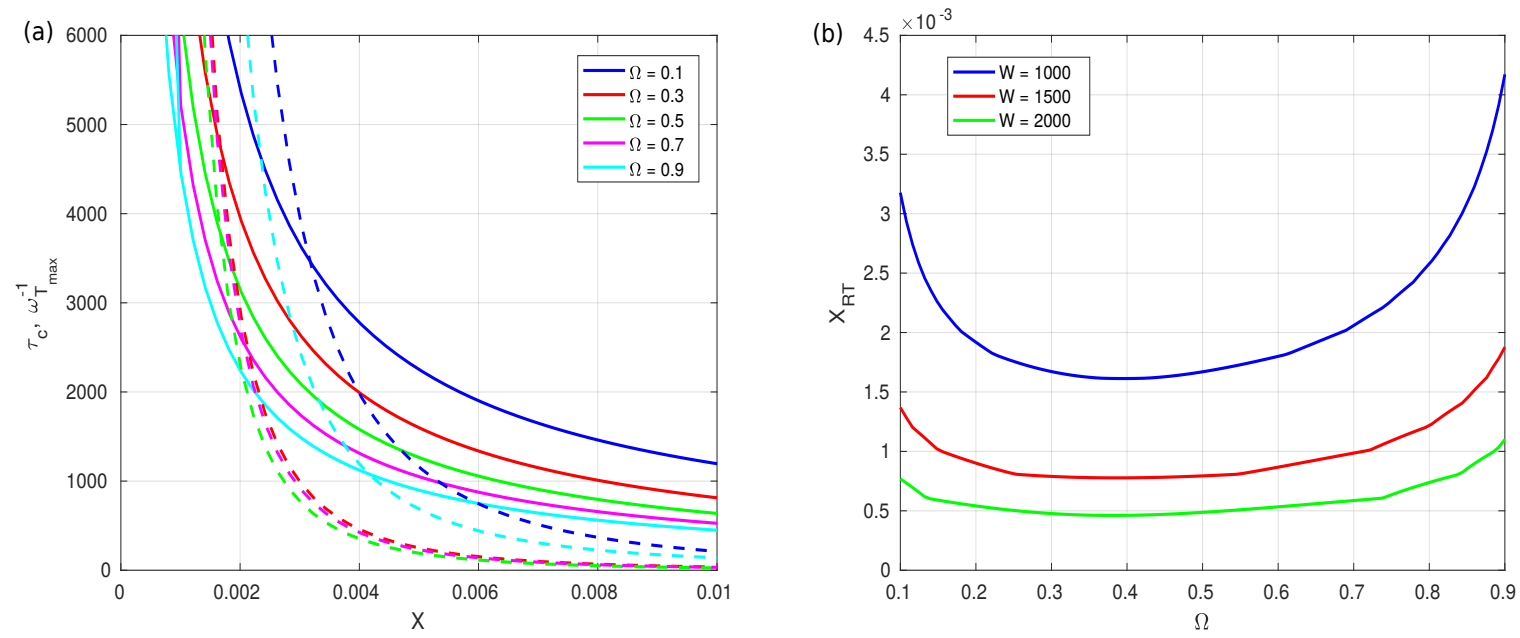

Figura 4.7 - (a) Tiempo de tránsito de una partícula fluida en la región de máxima aceleración (líneas contínuas) y tiempo de crecimiento de la inestabilidad de RayleighTaylor (líneas discontínuas) en función de la distancia $X$, para varios valores de $\Omega$ (con $\beta^{2} W^{-1} A_{w r} c_{d} / 4 \sin \theta_{c}=1$ y $\beta=10^{3}$ ). (b) Distancia $X_{R T}$ a la que se rompe la lámina por efecto de la inestabilidad de Rayleigh-Taylor en función de la frecuencia $\Omega$, para varios valores de $W\left(\operatorname{con} \alpha=10^{-3}\right.$ y $\left.A_{w r} c_{d} / 4 \sin \theta_{c}=10^{-3}\right)$.

\subsection{Inestabilidad centrífuga debida al giro del líquido}

Cerca de la salida del atomizador, antes de que las líneas de corriente se hagan rectas, la lámina líquida está sometida a una aceleración centrípeta debida al giro del líquido. Esta aceleración también puede dar lugar a una inestabilidad de Rayleigh-Taylor, que estudiaremos en esta sección usando un método cualitativo aproximado. Admitiendo que la componente de la aceleración normal a la lámina es $a_{\theta}=W^{-2} \gamma^{2} / r^{3}$, analizaremos separadamente la evolución de pequeñas perturbaciones de longitud de onda mucho mayor o mucho menor que el espesor de la lámina. 


\subsubsection{Perturbaciones de longitud de onda grande}

Consideraremos en primer lugar perturbaciones de longitud de onda grande frente al espesor local de la lámina, $k_{T} h \ll 1$, para las que es aplicable (4.56). El número de onda crítico por debajo del cual las perturbaciones son inestables es entonces $k_{c}=W a_{\theta}^{1 / 2} \sim$ $\gamma / r^{3 / 2}$ (ver (4.57)), mientras que el máximo ritmo de crecimiento de las perturbaciones es, de acuerdo con (4.58):

$$
\omega_{T_{\text {max }}}=W a\left(\frac{h}{2}\right)^{1 / 2} \sim W^{-1} \frac{\gamma^{2} h^{1 / 2}}{r^{3}}
$$

La distancia adimensional que recorre una perturbación durante su tiempo de crecimiento, considerando que su velocidad es del orden de la velocidad del fluido, es $L \sim \omega_{T_{\text {max }}}^{-1}$, luego:

$$
L \sim W \frac{r^{3}}{\gamma^{2} h^{1 / 2}}
$$

por lo que, teniendo en cuenta que $\gamma \sim 1 \mathrm{y} h \sim r^{-1}$ :

$$
\frac{L}{r} \sim W \frac{r^{2}}{\gamma^{2} h^{1 / 2}} \sim W r^{5 / 2}
$$

Así pues, la perturbación tiene tiempo de crecer si se origina a una distancia $r \ll W^{-2 / 5}$ del origen de la lámina, que es pequeña comparada con el tamaño de la lámina sin perturbar, $(r \sim 1)$. Además, en estas condiciones:

$$
k_{c} L \sim W \frac{r^{1 / 2}}{\gamma h^{1 / 2}} \sim W r \gg 1
$$

Lo que justifica el empleo de los resultados (4.55) - (4.58) de Keller \& Kolodner (1954) para una lámina plana de espesor constante. Sin embargo:

$$
k_{c} h \sim \frac{\gamma h}{r^{3 / 2}} \sim r^{-5 / 2} \gg 1
$$

que no es pequeño, contrariamente a lo supuesto más arriba. Esta inconsistencia muestra que las perturbaciones con $k_{T} h \ll 1$ no pueden crecer a causa de la inestabilidad centrífuga en la lámina generada por un atomizador.

\subsubsection{Perturbaciones de longitud de onda pequeña}

Consideremos ahora perturbaciones de longitud de onda pequeña frente al espesor local de la lámina, $k_{T} h \gg 1$, cuyo crecimiento originará gotas de tamaño pequeño frente a 
$h$. Para estas perturbaciones la relación de dispersión (4.55) se reduce al resultado clásico de Rayleigh:

$$
\omega_{T}^{2}=W^{-2} k_{T}^{3}\left(1-W^{2} \frac{a}{k_{T}^{2}}\right)
$$

cuyo valor máximo es:

$$
\omega_{T_{\max }} \sim W^{1 / 2} a^{3 / 4} \sim W^{-1} \frac{\gamma^{3 / 2}}{r^{9 / 4}}
$$

Así pues, para estas perturbaciones:

$$
\frac{L}{r} \sim \frac{\omega_{T_{\max }}^{-1}}{r} \sim W \frac{r^{5 / 4}}{\gamma^{3 / 2}} \sim W r^{5 / 4}
$$

Luego $L / r \ll 1$ si la perturbación se origina a una distancia $r \ll W^{-4 / 5}$ del origen de la lámina. Ahora, $k_{c} h \sim r^{-5 / 2} \gg 1 \mathrm{y}$ :

$$
k_{c} L \sim W \frac{r^{3 / 4}}{\gamma^{1 / 2}} \sim W r^{3 / 4} \gg 1
$$

Los ligamentos y gotas generados mediante esta inestabilidad tendrían un radio del orden de $k_{c}^{-1}$, esto es:

$$
k_{c}^{-1}=W^{-1} \frac{1}{\sqrt{a}} \sim \frac{r^{3 / 2}}{\gamma} \sim r^{3 / 2} \ll W^{-6 / 5}
$$

Esta inestabilidad afecta únicamente a la superficie exterior de la lámina líquida, pues para excitar la inestabilidad de Rayleigh-Taylor es necesario que la aceleración perpendicular a la entrefase esté dirigida hacia el fluido de mayor densidad. Ahora bien, en la lámina generada por un atomizador real, el líquido próximo a esta superficie proviene de la capa límite de la pared de la cámara y por tanto tiene una velocidad azimutal reducida, lo que dificulta el desarrollo de la inestabilidad centrífuga y puede hacer que la inestabilidad responsable de la rotura de la lámina sea la analizada en la Sección (4.3). 


\section{Capítulo 5}

\section{Conclusiones}

Los atomizadores de presión con giro son uno de los tipos más comunes de atomizador. Se usan en gran número de aplicaciones industriales, como hornos, turbinas de gas, maquinaria agrícola, extinción de incendios, entre otros. Estos atomizadores generan una lámina cónica giratoria de un líquido, que rápidamente se rompe en ligamentos y posteriormente en gotas. Para generar esta lámina, se inyecta el líquido en una cámara axilsimétrica a través de unos canales tangentes a la pared de dicha cámara, con el objetivo de proporcionar un movimiento de giro al fluido, en forma de un torbellino hueco con un núcleo de aire que se extiende a lo largo del eje de dicha cámara. Este giro produce un gradiente radial de presión que equilibra a las fuerzas centrífugas. Tras recorrer la cámara, el líquido converge hacia un orificio de salida concéntrico con el eje de la misma, y emerge formando una película giratoria anular que enseguida se vuelve cónica cuando su movimiento radial deja de estar restringido por las paredes del atomizador.

Las principales características del flujo en un atomizador son el espesor de la lámina que forma el líquido cuando sale del atomizador, el ángulo de la lámina cónica que se forma a cierta distancia aguas abajo, y el coeficiente de descarga, $c_{d}=Q /\left(\pi R_{0}^{2} \sqrt{2\left(p_{0}-p_{a}\right) / \rho}\right)$, donde $Q$ es el caudal inyectado en el atomizador, $R_{0}$ es el radio del orificio de salida, $p_{0}$ es la presión de remanso con que se inyecta el líquido en la cámara, $p_{a}$ es la presión del gas del ambiente y $\rho$ es la densidad del líquido. Todas estas características dependen del flujo giratorio en el interior de la cámara.

La variedad y las numerosas aplicaciones de los atomizadores de presión con giro hacen que la comprensión detallada de su funcionamiento, tanto en lo que se refiere al flujo interno como a la atomización y dispersión de los aerosoles que producen, sea de considerable interés, en particular con vistas a mejorar el diseño de estos dispositivos o a adaptarlos a aplicaciones concretas. 
Los problemas fluidodinámicos implicados son complejos. El pequeño tamaño de los atomizadores, las dificultades para el acceso óptico y la disparidad de escalas de velocidad (por ejemplo entre la velocidad azimutal y la meridional en la cámara del atomizador) complican los ensayos experimentales y limitan la información que se puede extraer de ellos. El cálculo numérico directo permite aliviar algunas de estas dificultades, pero no está libre de incertidumbres, que provienen en gran medida de la presencia simultánea de muchos procesos, cuya influencia sobre las características del atomizador no es fácil identificar en una simulación compleja. Por ejemplo, hay inestabilidades centrífugas, e incluso turbulencia en la cámara, que podrían no jugar un papel esencial, pero que deben calcularse ineludiblemente en una simulación directa, con el consiguiente aumento de complejidad.

En estas condiciones, la formulación de modelos aproximados de procesos o grupos de procesos individuales, que puedan analizarse con relativa facilidad y proporcionar información relevante, ha sido un modo fructífero de abordar estos problemas. De hecho, se ha obtenido mucha información sobre las características del flujo usando modelos irrotacionales (Abramovich 1944; Novikov 1948; Taylor 1948; Giffen \& Muraszew 1953; Nieuwkamp 1985). Estos modelos a menudo emplean una aproximación cuasi-unidireccional, en la que la velocidad en el orificio de salida es uniforme y sin componente radial, y el flujo a la salida es crítico, en el sentido de que su velocidad axial coincide con la velocidad de propagación de ondas en la lámina líquida. Mediante estos modelos irrotacionales se obtienen las características del flujo antes mencionadas en función de un único parámetro adimensional, la constante del atomizador, $\Delta=A_{i} /\left(D_{c} D_{0}\right)$, donde $A_{i}$ es el área total de los canales de entrada a la cámara, y $D_{c}=2 R_{c}$ y $D_{0}=2 R_{0}$ son los diámetros de la cámara y del orificio de salida, respectivamente. Este parámetro se puede expresar también en función del cociente del caudal entre el producto de la circulación por el radio del orificio de salida, $\Delta=Q /\left(4 \Gamma R_{0}\right)$.

Para estudiar el efecto de otros parámetros geométricos se ha construido un modelo numérico del problema irrotacional basado en el método de los elementos de contorno. Mediante el estudio de dicho modelo se han mostrado discrepancias en los resultados frente a los predichos por la teoría cuasi-unidireccional, debido a la presencia de una componente radial de la velocidad del fluido en el orificio de salida, lo que va en contra de las hipótesis de dicha teoría.

La sección interior del atomizador se estrecha al pasar de la cámara al orificio de salida, lo que origina un aumento de la velocidad axial del fluido. Este aumento de velocidad da lugar, como muestra la ecuación de Euler-Bernoulli, a una disminución de la presión en el 
líquido. Debido a ello, el diámetro del núcleo de aire es mayor a la salida del atomizador que en la cámara del mismo. Durante la transición que sufre el núcleo de aire en la región próxima al orificio de salida para variar de diámetro, se genera una componente radial de la velocidad del líquido orientada en sentido opuesto al eje de simetría. A medida que el líquido avanza por el orificio de salida, esta componente radial irá disminuyendo hasta que prácticamente desaparece cuando el diámetro del núcleo de aire alcanza el valor que le corresponde en esa sección del atomizador. Si el orificio de salida es lo suficientemente largo para que el núcleo de aire alcance este valor del diámetro, entonces el comportamiento del flujo será el correspondiente al calculado por la teoría cuasi-unidireccional, pues la velocidad no presentará componente en la dirección radial. Pero si el orificio de salida es más corto, el líquido abandonará el atomizador con una componente de velocidad radial que dará lugar a un aumento del ángulo que forma la lámina líquida con el eje de simetría a la salida del orificio. A medida que vaya aumentando la longitud de dicho orificio, más se aproximan los resultados a los de la teoría cuasi-unidireccional, hasta llegar a cierto valor a partir del cual el aumento de la longitud del orificio no tiene ningún efecto en las características del flujo.

En la región de transición entre la pared convergente y el orificio de salida el flujo está sometido a un giro, el cual tendrá asociada la aparición de una componente radial de la velocidad orientada hacia el eje de simetría. Esta velocidad radial será mayor cuanto mayor sea el ángulo de la pared convergente. Según avanza el fluido a lo largo del orificio de salida, esta componente radial irá disminuyendo, hasta desaparecer cuando el diámetro del núcleo de aire alcance el valor que le corresponde en esta región. Pero en el caso de que el orificio de salida no sea lo suficientemente largo, esta velocidad radial estará presente en el líquido cuando éste abandone el atomizador, lo que dará lugar a una disminución del ángulo que forma la lámina líquida con el eje de simetría. En el caso de que la transición entre la región convergente y el orificio de salida sea suave, el giro del flujo será menos brusco y el efecto antes descrito tiende a desaparecer.

Debido a la presencia de una superficie libre (la superficie del núcleo de aire) y de una fuerza másica aparente (la debida a la aceleración centrípeta), el flujo en el interior de un atomizador de presión con giro es un ambiente idóneo para la propagación de ondas. Varios autores ya observaron, mediante técnicas experimentales y numéricas, este fenómeno no estacionario (Donjat et al. 2003, Yule \& Chinn 2000, Maatje, von Lavante \& Albina 2002, von Lavante, Maatje \& Albina 2002). Un estudio de la propagación de ondas en la superficie del núcleo de aire ha permitido obtener las relaciones de dispersión correspondientes a las mismas. Para las ondas propagándose en el interior de la cámara del 
atomizador se ha obtenido que, para longitudes de onda menores que el radio del núcleo de aire, la velocidad de propagación de las ondas es $c \sim k^{-1 / 2}$, donde $k$ es el número de onda asociado a una cierta frecuencia, luego es un sistema dispersivo, pues la velocidad de fase de las ondas varía con la longitud de onda, y análogo al de las ondas gravitatorias en aguas profundas. Si la longitud de onda es mayor que el radio del núcleo de aire la velocidad de propagación de ondas es $c \sim k^{-1}(\ln (k))^{-1 / 2}$, que es un resultado específico de este problema y que representa también un sistema dispersivo. Para el caso de las ondas propagándose en la región próxima al orificio de salida, considerando longitudes de onda mayores que el radio del núcleo de aire, se obtiene que la velocidad de propagación de las ondas es independiente de la longitud de onda, luego se trata de ondas no dispersivas que se propagan sin deformarse, resultado análogo al del problema de propagación de ondas en aguas poco profundas.

En cuanto a la transmisión de ondas en la región de transición entre la cámara y el orificio de salida, se puede simplificar el análisis considerando que el cambio de sección es compacto, esto es, que el tamaño característico de la región de transición donde la sección cambia es pequeño es pequeño comparado con la longitud de onda característica. En esta situación, la onda incidente procedente de la cámara se reflejará al llegar a esta región, pero cederá una parte de su energía a la onda transmitida a la región próxima al orificio de salida, por lo que la onda reflejada tendrá ligeramente menos energía que la onda incidente. Esta última onda se propagará al final de la cámara y regresará hacia el orificio, cediendo otra parte de su energía. Así pues, mediante este mecanismo, la energía de la onda de la cámara se irá disipando.

Si la sección de la cámara es lentamente variable, entonces la región de transición entre la cámara y el orificio de salida no se puede considerar compacta. Para resolver este problema se ha propuesto una solución asintótica de manera similar al estudio realizado por Keller (1958) sobre la propagación de ondas en aguas de profundidad no uniforme. Mediante este estudio se concluye que el flujo de la acción de ondas se conserva a lo largo de la cámara, esto es, la relación entre el flujo de energía de las ondas y su frecuencia permanece constante. Debido a ello, para una frecuencia determinada, la energía de las ondas aumentará al disminuir la sección de la cámara, y en una sección concreta de la cámara, la energía de las ondas aumentará al hacerlo la frecuencia.

Sin embargo, si en el problema se consideran los efectos de la viscosidad del líquido, entonces se formarán capas límite en las paredes internas del atomizador. En estas capas límite la velocidad de giro del líquido se reduce, anulándose en las paredes. Ello produce una disminución de la aceleración centrípeta en el interior de las capas límite, donde 
también es impuesto el gradiente radial de presión que acompaña al giro del líquido, si la pared no es paralela al eje de la cámara. Así pues, éste último no puede ser equilibrado por la aceleración centrípeta, originando un movimiento del fluido hacia el eje de simetría en las paredes internas del atomizador que no son paralelas a dicho eje (bombeo de Ekman). En concreto, en el interior de la capa límite que se forma en la pared convergente situada entre la cámara y el orificio de salida el líquido será empujado hacia este último, y en la capa límite que se genera en la base de la cámara el líquido se mueve hacia el núcleo de aire hasta alcanzarlo, tras lo cual gira y fluye rodeándolo hacia el orificio de salida. De esta forma, estas capas límite llegan a la lámina líquida anular que se forma en el orificio de salida por sus superficies interior y exterior, y pueden ocupar un porcentaje considerable del espesor de la misma. Este hecho ya fue indicado por Taylor (1948), y fue confirmado por estudios posteriores (Binnie \& Harris 1950; Taylor 1950; Cooke 1952; Binnie, Hookings \& Kamel 1957; Burggraf, Stewartson \& Belcher 1971; Bloor \& Ingham 1977; Dumouchel et al. 1992), lo que supone una limitación en la validez de los modelos irrotacionales.

Además, como han observado otros autores mediante diferentes técnicas (Binnie et al. 1957; Jeng et al. 1998; Wang et al. 1999; Yule \& Chinn 2000; Donjat et al. 2003; NouriBorujerdi \& Kebriaee 2012) es posible la aparición de recirculación en el interior de la cámara del atomizador cuando los valores de la constante del atomizador no son altos. Sin embargo, a raíz de sus resultados, la presencia de esta recirculación no da lugar a efectos importantes en las características del flujo. También se ha observado la formación de torbellinos de Görtler en la pared cóncava de la cámara (Yule \& Chinn 2000; Maatje, von Lavante \& Albina 2002; von Lavante, Maatje \& Albina 2002), localizados en la región cilíndrica que precede a la región convergente, y debidos a la presencia de inestabilidades centrífugas en el flujo giratorio en la capa límite sobre dicha pared (Rayleigh 1916).

Para estudiar el problema viscoso se ha desarrollado un modelo basado en una aproximación cuasi-cilíndrica. Este modelo permite reducir considerablemente el coste que supone realizar una simulación numérica basada en las ecuaciones de Navier-Stokes completas, y proporciona las principales características del flujo en el interior del atomizador. Sin embargo, este modelo presenta algunas limitaciones. Se puede dar cuenta de una recirculación moderada, pero el modelo falla cuando el tamaño de la región de recirculación aumenta. Para prevenir la formación de inestabilidades centrífugas, se ha sustituido la pared cilíndrica de la cámara por una ligeramente cónica y convergente, de forma que sobre la misma se genere bombeo de Ekman que dé lugar a una velocidad axial que barra las perturbaciones. Además, la aproximación cuasi-cilíndrica no es capaz de describir el 
flujo en la región próxima a los canales de entrada y en la capa límite generada en la base de la cámara. Por ello se han impuesto unas condiciones a la entrada que aproximan el flujo real, consistentes en unos perfiles de velocidad que consideran el exceso de velocidad axial y el defecto de velocidad azimutal en la capa límite que rodea al núcleo de aire. Para la condición de contorno a la salida se ha impuesto que el flujo sea crítico al final del orificio. Finalmente, se ha propuesto una aproximación para el valor del semiángulo del cono que forma la lámina líquida a la salida similar a las propuestas por otros autores (Datta \& Som 2000; Yule \& Chinn 2000; Nouri-Borujerdi \& Kebriaee 2012). Los resultados obtenidos con este modelo han sido comparados con los presentes en la literatura previa, mostrando un buen acuerdo con los mismos.

El bombeo de Ekman en las capas límite induce una velocidad axial en las mismas que da cuenta de parte del caudal. Debido a ello, la sobrepresión requerida en la cámara es menor que la obtenida por la teoría irrotacional, por lo que el coeficiente de descarga es mayor. La diferencia entre ambos resultados es mayor cuando disminuye la constante del atomizador, pues la fracción del caudal que está en las capas límite aumenta. Así pues, el espesor de las capas límite aumenta al disminuir la constante del atomizador, y llegan a cubrir un porcentaje importante del espesor de la lámina líquida a la salida para valores bajos del mismo. También es mayor el valor obtenido para el espesor de la lámina líquida al final del orificio de salida que el calculado por la teoría irrotacional, debido a la presencia de la capa límite alrededor del núcleo de aire. En cuanto a la variación del semiángulo de la lámina cónica con respecto a la constante del atomizador se obtienen resultados similares a los del flujo irrotacional, excepto para valores pequeños de dicha constante, para los cuales aumenta el espesor de las capas límite, en las cuales el líquido presenta una velocidad azimutal reducida, y el valor del semiángulo disminuye.

Las variaciones calculadas en las características del flujo debidas a otros parámetros geométricos aparte de la constante del atomizador son pequeñas en comparación con las debidas a ésta última. La fracción del flujo en la capa límite alrededor del núcleo de aire aumenta cuando disminuye el radio de la cámara, por la forma en que han sido definidas las condiciones a la entrada, con lo que la sobrepresión necesaria es menor. Así pues, el coeficiente de descarga aumenta cuando disminuye el radio de la cámara. También lo hace el espesor de la lámina líquida en el orificio de salida, pero menos que el espesor de la capa límite, por lo que la fracción del espesor de la lámina ocupado por la capa límite aumenta. A su vez, debido al incremento del espesor de la capa límite alrededor del núcleo de aire, el semiángulo de la lámina cónica disminuye cuando lo hace el radio de la cámara, debido a la menor velocidad de giro a la salida. En cuanto a la capa límite generada sobre la 
pared de la cámara, su espesor aumenta cuando lo hace el radio de la misma, lo que da lugar a un ligero aumento de la fracción del espesor de la lámina ocupada por las capas límite cuando lo hace el radio de la cámara, así como a una disminución del semiángulo de la lámina cónica. Además, para valores altos del radio de la cámara aparece recirculación en la capa límite cuando entra en la parte convergente de la pared.

Al aumentar la longitud del orificio de salida también aumenta el espesor de la capa límite en dicha región, con la consiguiente disminución de la velocidad de giro y, por consiguiente, del semiángulo de la lámina cónica. Debido a este aumento del espesor de la capa límite, la sobrepresión en la cámara ha de ser mayor para mantener el caudal, por lo que disminuye el coeficiente de descarga. Esta mayor sobrepresión que actúa en el líquido fuera de la capa límite origina un incremento de la velocidad a la salida, lo que da lugar a una disminución del espesor de la lámina líquida en el orificio. De forma similar, al aumentar la longitud de la región casi-cilíndrica de la cámara, aumenta el espesor de la capa límite en la pared de la misma, lo que da lugar a una disminución del coeficiente de descarga y del semiángulo de la lámina cónica. Sin embargo, el espesor de la lámina líquida en el orificio de salida aumenta, pues el incremento de espesor de la capa límite en esta región supera el efecto del ligero aumento de la velocidad fuera de la misma.

La componente radial de la velocidad del líquido deja de estar restringida por las paredes del inyector una vez que la lámina líquida anular abandona el orificio de salida. Debido a ello la lámina comienza a diverger radialmente y, a cierta distancia del inyector, se rompe en ligamentos y posteriormente en gotas. El espesor de dicha lámina, el ángulo que forma aguas abajo del inyector y la velocidad del líquido dependen del flujo dentro de la cámara, que es función de la constante del atomizador y de la geometría del mismo. A la salida del inyector, debido a que el número de Weber $W=\rho U_{\infty}^{2} R_{0} / \sigma$ es grande (donde $\left.U_{\infty}=\sqrt{2\left(p_{0}-p_{a}\right) / \rho}\right)$, se puede despreciar el efecto de la tensión superficial $\sigma$ y la lámina líquida toma una forma cónica, si la distancia a la que se rompe es suficientemente grande. Pero el efecto de la tensión superficial crece cuando la lámina líquida diverge, y puede cambiar la forma cónica de la misma a una cierta distancia $r_{c}$, a la cual el espesor es $h_{c} \sim R_{0}^{2} / r_{c}$. Esta distancia se ha estimado considerando que para ella la fuerza resultante debida al salto de presión a través de las superficies de la lámina, $\sigma / r_{c}$, cause variaciones apreciables del flujo de cantidad de movimiento, esto es, $\sigma / r_{c} \sim \rho U_{\infty}^{2} h_{c} / r_{c}$, resultando $r_{c} \sim W R_{0}$ y $h_{c} \sim W^{-1} R_{0}$.

El proceso de desintegración de la lámina mediante el cual se generan ligamentos que posteriormente se rompen en gotas tiene lugar en varias etapas. Cuando la velocidad relativa entre el líquido y el gas que lo rodea es suficientemente alta se excita una inestabilidad 
de Kelvin-Helmholtz, la cual produce una oscilación lateral de la lámina. La presencia de una perturbación que produzca un pequeño desplazamiento no estacionario $\eta$ de la superficie de la lámina da lugar a velocidades en el gas que llevan asociadas variaciones de presión en el mismo. En las regiones donde $\eta>0$ se producirá una disminución de la presión en el gas, y al revés donde $\eta<0$, lo que da lugar a la aparición de una aceleración del líquido normal a la lámina que origina el crecimiento de las perturbaciones. Se ha calculado el tiempo característico del crecimiento de las perturbaciones, $t_{2} \sim h U_{\infty}^{-1} \alpha^{-1 / 2}(\lambda / h)^{1 / 2}$, donde $h$ es el espesor local de la lámina, $\alpha$ es la relación entre las densidades del gas y del líquido, y $\lambda$ es la longitud de onda de la perturbación. De esta expresión se extrae que el crecimiento de las perturbaciones será más rápido cuando la longitud de onda sea más corta. Pero el crecimiento de las perturbaciones está dificultado por la fuerza que ejerce la tensión superficial, y que es mayor cuanto menor sea la longitud de onda. Así pues, hay una longitud de onda crítica $\lambda_{c}$ por debajo de la cual los efectos de la tensión superficial son dominantes y las perturbaciones no crecen. El valor calculado para esta longitud de onda crítica es $\lambda_{c} \sim R_{0} /(\alpha W)$. También se ha obtenido la distancia $L$ a la que se produce el crecimiento de las perturbaciones, $L / r_{c} \sim\left(\alpha W^{2}\right)^{-2 / 3}$, de donde se extrae que este crecimiento se produce antes de alcanzar el límite de la lámina sin perturbar si $\alpha W^{2} \gg 1$, y tendrá lugar a una distancia tanto más próxima a la salida del atomizador cuanto mayor sea el número de Weber W.

La inestabilidad de la lámina líquida se ha estudiado considerando que las perturbaciones se desarrollan a una distancia de la salida del atomizador $r \ll W R_{0}$, por lo que $r \ll r_{c}$ y la forma de la lámina sin perturbar es prácticamente cónica. Se han introducido perturbaciones de pequeña amplitud a la solución estacionaria del problema. La longitud de onda de dichas perturbaciones es larga en comparación con el espesor de la lámina, pero corta en comparación con la extensión de la misma. Así pues, la variación de la amplitud con la distancia es lenta, por lo que se han resuelto las ecuaciones linealizadas que gobiernan el problema perturbado empleando un método WKB. De esta forma se ha obtenido una relación de dispersión, la cual, para una frecuencia de excitación dada, permite obtener el número de onda de la perturbación en función de la distancia a la salida del atomizador. Esta relación de dispersión ha permitido ver que, para los modos inestables, la longitud de onda de la perturbación disminuye según ésta se aleja del atomizador, mientras que la velocidad de propagación de las ondas respecto al fluido es nula a la salida del atomizador y va aumentando según las ondas se alejan. Estos resultados están de acuerdo con los obtenidos experimentalmente por Bremond, Clanet \& Villermaux (2007). Además, se ha obtenido la evolución de la amplitud de las perturbaciones, 
resultando ésta creciente con la distancia a la salida del atomizador.

La inestabilidad de Kelvin-Helmholtz da lugar a aceleraciones transitorias de las partículas fluidas perpendiculares a la lámina líquida, y pueden derivar en una inestabilidad secundaria de Rayleigh-Taylor, en el caso de que la amplitud de las perturbaciones y su velocidad relativa al fluido sean lo suficientemente altas. Esta inestabilidad da lugar a variaciones en el espesor de la lámina líquida, particularmente en la dirección azimutal, las cuales se amplificarán si la inestabilidad tiene tiempo de desarrollarse. Estas modulaciones en el espesor de la lámina son las causantes de la formación de ligamentos longitudinales que posteriormente se rompen en gotas. Para que esto suceda, el tiempo de tránsito de las partículas fluidas por las inmediaciones de una cresta de la onda, que es donde la aceleración perpendicular a la lámina es mayor, ha de ser superior al tiempo característico de crecimiento de la inestabilidad de Rayleigh-Taylor. Este tiempo característico es inversamente proporcional a la aceleración perpendicular a la lámina a la que están sometidas las partículas, la cual es a su vez proporcional a la amplitud de las perturbaciones. Así pues, como esta amplitud aumenta con la distancia a la salida del atomizador, el tiempo característico para que se produzca la inestabilidad disminuye con dicha distancia. Por otra parte, la distancia recorrida por una partícula fluida en la región de máxima aceleración, en torno a una cresta de la onda, es del orden de la longitud de onda, mientras que la velocidad con que lo hace es la velocidad relativa entre el fluido y la onda. De esta forma, el cociente entre las dos da el orden del tiempo de tránsito de la partícula por una cresta. La longitud de onda decrece con la distancia, y la velocidad relativa entre el fluido y la onda parte de cero y aumenta según la onda se aleja del atomizador, por lo que el tiempo de tránsito de la partícula por una cresta también será una función decreciente con la distancia. El tiempo característico de la inestabilidad es inicialmente superior al tiempo de tránsito de las partículas por una cresta, pero a medida que la onda se aleja del atomizador la diferencia entre ambos se reduce, y la inestabilidad de Rayleigh-Taylor se origina cuando ambos tiempos son del mismo orden. De esta forma se ha podido determinar la distancia a la que la lámina líquida se rompe en ligamentos que dan lugar a la formación de gotas.

Otra posible forma en que se pudiera presentar la inestabilidad de Rayleigh-Taylor es por la presencia de la aceleración centrípeta debida al giro del fluido, la cual tiene una componente perpendicular a la lámina líquida, y que afectará únicamente a la superficie exterior de la lámina líquida, pues para que la entrefase entre dos fluidos sea inestable la aceleración del líquido ha de ir dirigida hacia el fluido de mayor densidad. Pero la velocidad azimutal del fluido presente en las proximidades de la superficie exterior de la 
lámina es baja, debido a la capa límite que se origina en la pared de la cámara, lo que dificulta que se origine dicha inestabilidad de esta manera. 


\section{Apéndice A}

\section{Propagación de ondas en la superficie del núcleo de aire}

En este Apéndice se incluye el desarrollo del análisis de propagación de ondas en la superficie del núcleo de aire presentado en la Sección 2.5. Llamamos $r_{s}$ y $\varphi_{s_{p}}$ a los valores del radio del núcleo de aire y de las perturbaciones en el potencial de velocidades en la cámara, y $r_{0}$ y $\varphi_{0_{p}}$ a los valores correspondientes cerca del orificio de salida, de radio $R_{0}$. El problema a resolver viene dado por las siguientes ecuaciones en el interior de la cámara:

$$
\begin{gathered}
\frac{\partial^{2} \varphi_{s_{p}}}{\partial x^{2}}+\frac{1}{r} \frac{\partial}{\partial r}\left(r \frac{\partial \varphi_{s_{p}}}{\partial r}\right)=0 \\
\frac{\partial r_{s}}{\partial t}+\frac{\partial \varphi_{s_{p}}}{\partial x} \frac{\partial r_{s}}{\partial x}-\frac{\partial \varphi_{s_{p}}}{\partial r}=0 \quad \text { en } \quad r=r_{s} \\
\frac{\partial \varphi_{s_{p}}}{\partial t}+\frac{1}{2}\left(\frac{\partial \varphi_{s_{p}}}{\partial x}\right)^{2}+\frac{1}{2}\left(\frac{\partial \varphi_{s_{p}}}{\partial r}\right)^{2}+\frac{\Gamma^{2}}{8 \pi^{2} r_{s}^{2}}+\frac{p_{a}}{\rho}=C_{s}(t) \quad \text { en } \quad r=r_{s} \\
\nabla \varphi_{s_{p}} \rightarrow 0 \quad \text { en } \quad r \rightarrow \infty
\end{gathered}
$$

mientras que en la región en torno al orificio de salida se tiene:

$$
\begin{gathered}
\frac{\partial^{2} \varphi_{0_{p}}}{\partial x^{2}}+\frac{1}{r} \frac{\partial}{\partial r}\left(r \frac{\partial \varphi_{0_{p}}}{\partial r}\right)=0 \\
\frac{\partial r_{0}}{\partial t}+\left(u_{0_{s t}}+\frac{\partial \varphi_{0_{p}}}{\partial x}\right) \frac{\partial r_{0}}{\partial x}-\frac{\partial \varphi_{0_{p}}}{\partial r}=0 \quad \text { en } \quad r=r_{0} \\
\frac{\partial \varphi_{0_{p}}}{\partial t}+\frac{1}{2}\left(u_{0_{s t}}+\frac{\partial \varphi_{0_{p}}}{\partial x}\right)^{2}+\frac{1}{2}\left(\frac{\partial \varphi_{0_{p}}}{\partial r}\right)^{2}+\frac{\Gamma^{2}}{8 \pi^{2} r_{0}^{2}}+\frac{p_{a}}{\rho}=C_{0}(t) \quad \text { en } \quad r=r_{0} \\
\frac{\partial \varphi_{0_{p}}}{\partial r}=0 \quad \text { en } \quad r=R_{0}
\end{gathered}
$$


La constante $C(t)$ de la ecuación de Euler-Bernoulli se hace igual a su valor en ausencia de perturbaciones, admitiendo que su variación respecto a este valor se considera incluida en el potencial de velocidades. Así, si es $\Delta C(t)$ la variación de $C$, basta redefinir dicho potencial de velocidades añadiéndole $\int_{0}^{t} \Delta C(t) \mathrm{d} t$. Así pues:

$$
C_{s}=\frac{\Gamma^{2}}{8 \pi^{2} r_{s_{s t}}^{2}}+\frac{p_{a}}{\rho} \quad ; \quad C_{0}=\frac{1}{2} u_{0_{s t}}^{2}+\frac{\Gamma^{2}}{8 \pi^{2} r_{0_{s t}}^{2}}+\frac{p_{a}}{\rho}
$$

donde $r_{s_{s t}}$ y $r_{0_{s t}}$ son los valores que toma el radio del núcleo de aire en la cámara y cerca del orificio de salida, respectivamente, y $u_{0_{s t}}$ es la velocidad axial en esta última región, en régimen estacionario. Escalando las variables espaciales con $R_{0}$, las velocidades con $\Gamma /\left(2 \pi R_{0}\right)$, las presiones con $\rho \Gamma^{2} /\left(4 \pi^{2} R_{0}^{2}\right)$ y el tiempo con $2 \pi R_{0}^{2} / \Gamma$, donde $\Gamma$ es la circulación y $\rho$ la densidad del líquido, las ecuaciones (A.1) - (A.4) quedan, en variables adimensionales:

$$
\begin{gathered}
\frac{\partial^{2} \varphi_{s_{p}}}{\partial x^{2}}+\frac{1}{r} \frac{\partial}{\partial r}\left(r \frac{\partial \varphi_{s_{p}}}{\partial r}\right)=0 \\
\frac{\partial r_{s}}{\partial t}+\frac{\partial \varphi_{s_{p}}}{\partial x} \frac{\partial r_{s}}{\partial x}-\frac{\partial \varphi_{s_{p}}}{\partial r}=0 \quad \text { en } r=r_{s} \\
\frac{\partial \varphi_{s_{p}}}{\partial t}+\frac{1}{2}\left(\frac{\partial \varphi_{s_{p}}}{\partial x}\right)^{2}+\frac{1}{2}\left(\frac{\partial \varphi_{s_{p}}}{\partial r}\right)^{2}+\frac{1}{2 r_{s}^{2}}=\frac{1}{2 r_{s_{s t}}^{2}} \quad \text { en } r=r_{s} \\
\nabla \varphi_{s_{p}} \rightarrow 0 \text { en } r \rightarrow \infty
\end{gathered}
$$

mientras que las ecuaciones (A.5) - (A.8) quedan:

$$
\begin{gathered}
\frac{\partial^{2} \varphi_{0_{p}}}{\partial x^{2}}+\frac{1}{r} \frac{\partial}{\partial r}\left(r \frac{\partial \varphi_{0_{p}}}{\partial r}\right)=0 \\
\frac{\partial r_{0}}{\partial t}+\left(u_{0_{s t}}+\frac{\partial \varphi_{0_{p}}}{\partial x}\right) \frac{\partial r_{0}}{\partial x}-\frac{\partial \varphi_{0_{p}}}{\partial r}=0 \quad \text { en } \quad r=r_{0} \\
\frac{\partial \varphi_{0_{p}}}{\partial t}+\frac{1}{2}\left(\frac{\partial \varphi_{0_{p}}}{\partial x}\right)^{2}+u_{0_{s t}} \frac{\partial \varphi_{0_{p}}}{\partial x}+\frac{1}{2}\left(\frac{\partial \varphi_{0_{p}}}{\partial r}\right)^{2}+\frac{1}{2 r_{0}^{2}}=\frac{1}{2 r_{0_{s t}}^{2}} \quad \text { en } \quad r=r_{0} \\
\frac{\partial \varphi_{0_{p}}}{\partial r}=0 \quad \text { en } \quad r=1
\end{gathered}
$$

Linealizando las ecuaciones (A.9) - (A.12), esto es, despreciando en las mismas los términos cuadráticos o de orden superior en las variables perturbadas:

$$
\begin{gathered}
\frac{\partial^{2} \varphi_{s_{p}}}{\partial x^{2}}+\frac{1}{r} \frac{\partial}{\partial r}\left(r \frac{\partial \varphi_{s_{p}}}{\partial r}\right)=0 \\
\frac{\partial \eta_{s}}{\partial t}-\frac{\partial \varphi_{s_{p}}}{\partial r}=0 \quad \text { en } \quad r=r_{s_{s t}}
\end{gathered}
$$




$$
\begin{gathered}
\frac{\partial \varphi_{s_{p}}}{\partial t}-\frac{\eta_{s}}{r_{s_{s t}}^{3}}=0 \quad \text { en } \quad r=r_{s_{s t}} \\
\nabla \varphi_{s_{p}} \rightarrow 0 \quad \text { en } \quad r \rightarrow \infty
\end{gathered}
$$

Haciendo lo mismo con las ecuaciones (A.13) - (A.16):

$$
\begin{gathered}
\frac{\partial^{2} \varphi_{0_{p}}}{\partial x^{2}}+\frac{1}{r} \frac{\partial}{\partial r}\left(r \frac{\partial \varphi_{0_{p}}}{\partial r}\right)=0 \\
\frac{\partial \eta_{0}}{\partial t}+u_{0_{s t}} \frac{\partial \eta_{0}}{\partial x}-\frac{\partial \varphi_{0_{p}}}{\partial r}=0 \quad \text { en } \quad r=r_{0_{s t}} \\
\frac{\partial \varphi_{0_{p}}}{\partial t}+u_{0_{s t}} \frac{\partial \varphi_{0_{p}}}{\partial x}-\frac{\eta_{0}}{r_{0_{s t}}^{3}}=0 \quad \text { en } \quad r=r_{0_{s t}} \\
\frac{\partial \varphi_{0_{p}}}{\partial r}=0 \quad \text { en } \quad r=1
\end{gathered}
$$

Se pueden eliminar las variables $\eta_{s}$ y $\eta_{0}$ combinando la ecuación (A.18) con (A.19) y la ecuación (A.22) con (A.23), con lo que el problema queda en términos de los potenciales de velocidades $\varphi_{s_{p}}$ y $\varphi_{0_{p}}$ únicamente. Las condiciones de contorno en la superficie del núcleo de aire son entonces:

$$
\frac{\partial^{2} \varphi_{s_{p}}}{\partial t^{2}}-\frac{1}{r_{s_{s t}}^{3}} \frac{\partial \varphi_{s_{p}}}{\partial r}=0 \quad \text { en } \quad r=r_{s_{s t}}
$$

en el primer caso, y

$$
\frac{\partial^{2} \varphi_{0_{p}}}{\partial t^{2}}+2 u_{0_{s t}} \frac{\partial^{2} \varphi_{0_{p}}}{\partial t \partial x}+u_{0_{s t}}^{2} \frac{\partial^{2} \varphi_{0_{p}}}{\partial x^{2}}-\frac{1}{r_{0_{s t}}^{3}} \frac{\partial \varphi_{0_{p}}}{\partial r}=0 \quad \text { en } \quad r=r_{0_{s t}}
$$

en el segundo. Se puede usar la transformada de Fourier en $x$ para expresar la solución en términos de ondas sinusoidales en cada una de las dos regiones. Además, la solución para cada componente de Fourier depende exponencialmente del tiempo, debido a que los coeficientes de las ecuaciones del problema lineal son independientes del tiempo. Escribiremos:

$$
\varphi_{s_{p}}=\hat{\varphi}_{s}(r) e^{-i k_{s} x+i \omega t}+c c \quad ; \quad \varphi_{0_{p}}=\hat{\varphi}_{0}(r) e^{-i k_{0} x+i \omega t}+c c
$$

donde $c c$ indica complejo conjugado, y $k_{s}$ y $k_{0}$ son los números de onda asociados a una cierta frecuencia $\omega$ mediante unas relaciones de dispersión que se calcularán más adelante. Las deformaciones de la superficie libre pueden expresarse como:

$$
\eta_{s}=\hat{\eta}_{s} e^{-i k_{s} x+i \omega t}+c c \quad ; \quad \eta_{0}=\hat{\eta}_{0} e^{-i k_{0} x+i \omega t}+c c
$$


Sustituyendo (A.27) en la ecuación de Laplace (A.17), y en las condiciones de contorno (A.25) y (A.20) se obtiene:

$$
\begin{gathered}
\frac{1}{r} \frac{\mathrm{d}}{\mathrm{d} r}\left(r \frac{\mathrm{d} \hat{\varphi}_{s}}{\mathrm{~d} r}\right)-k_{s}^{2} \hat{\varphi}_{s}=0 \\
-\omega^{2} \hat{\varphi}_{s}-\frac{1}{r_{s_{s t}}^{3}} \frac{\mathrm{d} \hat{\varphi}_{s}}{\mathrm{~d} r}=0 \quad \text { en } \quad r=r_{s_{s t}} \\
\hat{\varphi}_{s} \rightarrow 0 \quad \text { en } \quad r \rightarrow \infty
\end{gathered}
$$

Haciendo lo mismo en la ecuación de Laplace (A.21), y en las condiciones de contorno (A.26) y (A.24):

$$
\begin{gathered}
\frac{1}{r} \frac{\mathrm{d}}{\mathrm{d} r}\left(r \frac{\mathrm{d} \hat{\varphi}_{0}}{\mathrm{~d} r}\right)-k_{0}^{2} \hat{\varphi}_{0}=0 \\
-\left(\omega-u_{0_{s t}} k_{0}\right)^{2} \hat{\varphi}_{0}-\frac{1}{r_{0_{s t}}^{3}} \frac{\mathrm{d} \hat{\varphi}_{0}}{\mathrm{~d} r}=0 \quad \text { en } \quad r=r_{0_{s t}} \\
\frac{\mathrm{d} \hat{\varphi}_{0}}{\mathrm{~d} r}=0 \quad \text { en } \quad r=1
\end{gathered}
$$

Las soluciones generales de las ecuaciones (A.29) y (A.32) son:

$$
\begin{aligned}
& \hat{\varphi}_{s}=a_{s} K_{0}\left(k_{s} r\right)+b_{s} I_{0}\left(k_{s} r\right) \\
& \hat{\varphi}_{0}=a_{0} K_{0}\left(k_{0} r\right)+b_{0} I_{0}\left(k_{0} r\right)
\end{aligned}
$$

donde las funciones $I_{0}$ y $K_{0}$ son las funciones de Bessel modificadas de orden cero de primera y segunda especie, respectivamente, y las constantes $a_{s}, b_{s}, a_{0}$ y $b_{0}$ se determinarán imponiendo las condiciones de contorno correspondientes.

\section{A.1 Ondas en la cámara}

Teniendo en cuenta la condición de contorno (A.31), y que la función $I_{0}$ diverge en el infinito, ha de cumplirse $b_{s}=0$, luego (A.35) se reduce a:

$$
\hat{\varphi}_{s}=a_{s} K_{0}\left(k_{s} r\right)
$$

Sustituyendo (A.37) en (A.30):

$$
-\omega^{2} a_{s} K_{0}\left(k_{s} r_{s_{s t}}\right)-\frac{k_{s} a_{s}}{r_{s_{s t}}^{3}} K_{0}^{\prime}\left(k_{s} r_{s_{s t}}\right)=0
$$

Operando en la anterior expresión se llega a la siguiente relación de dispersión:

$$
\omega^{2}=-\frac{k_{s}}{r_{s_{s t}}^{3}} \frac{K_{0}^{\prime}\left(k_{s} r_{s_{s t}}\right)}{K_{0}\left(k_{s} r_{s_{s t}}\right)}
$$


En el caso de longitudes de onda grandes frente al radio del núcleo de aire, $k_{s} r_{s_{s t}} \ll 1$, la función de Bessel $K_{0}$ puede aproximarse de la siguiente manera:

$$
K_{0}\left(k_{s} r_{s_{s t}}\right) \approx-\ln \left(\frac{k_{s} r_{s_{s t}}}{2}\right)-\gamma
$$

cuya derivada es

$$
K_{0}^{\prime}\left(k_{s} r_{s_{s t}}\right) \approx-\frac{1}{k_{s} r_{s_{s t}}}
$$

donde $\gamma$ es la constante de Euler-Mascheroni, de valor aproximado $\gamma \approx 0,5772$. La relación de dispersión (A.38) se reduce entonces a:

$$
\omega^{2}\left[-\ln \left(\frac{k_{s} r_{s_{s t}}}{2}\right)-\gamma\right]=\frac{1}{r_{s_{s t}}^{4}}
$$

En el caso contrario, cuando $k_{s} r_{s_{s t}} \gg 1$, se tiene la siguiente aproximación para la función de Bessel $K_{0}$ :

$$
K_{0}\left(k_{s} r_{s_{s t}}\right) \approx \sqrt{\frac{\pi}{2 k_{s} r_{s_{s t}}}} e^{-k_{s} r_{s t}}
$$

cuya derivada es

$$
K_{0}^{\prime}\left(k_{s} r_{s_{s t}}\right) \approx-\sqrt{\frac{\pi}{2 k_{s} r_{s_{s t}}}} e^{-k_{s} r_{s_{s t}}}
$$

por lo que la relación de dispersión (A.38) puede expresarse como:

$$
\omega^{2}=\frac{k_{s}}{r_{s_{s t}}^{3}}
$$

\section{A.2 Ondas cerca del orificio de salida}

Sustituyendo la solución general de la ecuación de Bessel modificada (A.36) en la condición de contorno (A.33):

$$
a_{0} K_{0}^{\prime}\left(k_{0}\right)+b_{0} I_{0}^{\prime}\left(k_{0}\right)=0
$$

Despejando $b_{0}$ de (A.41):

$$
b_{0}=-a_{0} \frac{K_{0}^{\prime}\left(k_{0}\right)}{I_{0}^{\prime}\left(k_{0}\right)}
$$

Sustituyendo (A.42) en (A.36) se obtiene el potencial de velocidades:

$$
\hat{\varphi}_{0}=a_{0}\left(\frac{K_{0}\left(k_{0} r\right)}{K_{0}^{\prime}\left(k_{0}\right)}-\frac{I_{0}\left(k_{0} r\right)}{I_{0}^{\prime}\left(k_{0}\right)}\right)
$$


en donde se ha redefinido el valor constante $a_{0} K_{0}^{\prime}\left(k_{0}\right)$ como $a_{0}$. Insertando (A.43) en (A.32) se llega a la siguiente relación de dispersión:

$$
\left(\omega-u_{0_{s t}} k_{0}\right)^{2}=-\frac{k_{0}}{r_{0_{s t}}^{3}} \frac{K_{0}^{\prime}\left(k_{0} r_{0_{s t}}\right) I_{0}^{\prime}\left(k_{0}\right)-K_{0}^{\prime}\left(k_{0}\right) I_{0}^{\prime}\left(k_{0} r_{0_{s t}}\right)}{K_{0}\left(k_{0} r_{0_{s t}}\right) I_{0}^{\prime}\left(k_{0}\right)-K_{0}^{\prime}\left(k_{0}\right) I_{0}\left(k_{0} r_{0_{s t}}\right)}
$$

En el límite $\left(k_{0} r_{0_{s t}}, k_{0}\right) \ll 1$, la función de Bessel $I_{0}$ puede aproximarse de la siguiente manera:

$$
I_{0}\left(k_{0} r_{0_{s t}}\right) \approx 1+\frac{\left(k_{0} r_{0_{s t}}\right)^{2}}{4}
$$

cuya derivada es

$$
I_{0}^{\prime}\left(k_{0} r_{0 s t}\right) \approx \frac{k_{0} r_{0_{s t}}}{2}
$$

Haciendo uso de las aproximaciones para las funciones de Bessel modificadas para este límite en (A.44), y despreciando los términos de mayor orden, se llega a la siguiente expresión para la relación de dispersión:

$$
\left(\omega-u_{0_{s t}} k_{0}\right)^{2}=k_{0}^{2} \frac{1-r_{0_{s t}}^{2}}{2 r_{0_{s t}}^{4}}
$$

En el caso en que $k_{0} r_{0_{s t}} \ll 1$, pero $k_{0} \sim O(1)$, podemos despreciar el segundo sumando del numerador de la expresión de la relación de dispersión (A.44), por lo que la misma se reduce a:

$$
\left(\omega-u_{0_{s t}} k_{0}\right)^{2}=\frac{1 / r_{0_{s t}}^{4}}{-\left[\ln \left(\frac{k_{0} r_{0 s t}}{2}\right)+\gamma\right]-K_{0}^{\prime}\left(k_{0}\right) / I_{0}^{\prime}\left(k_{0}\right)}
$$




\section{Apéndice B}

\section{Propagación de ondas en la superficie del núcleo de aire a través de una región de transición compacta}

En este Apéndice se incluye el desarrollo del análisis de propagación de ondas en la superficie del núcleo de aire a través de una región de transición compacta presentado en la Sección 2.5. Se han escalado las variables espaciales con $R_{0}$, las velocidades con $\Gamma /\left(2 \pi R_{0}\right)$, las presiones con $\rho \Gamma^{2} /\left(4 \pi^{2} R_{0}^{2}\right)$ y el tiempo con $2 \pi R_{0}^{2} / \Gamma$, donde $R_{0}$ es el radio del orificio de salida, $\Gamma$ es la circulación y $\rho$ la densidad del líquido. Para calcular la amplitud de la onda reflejada se aplicará la conservación de la energía. Esto es, de acuerdo con Lighthill (1978):

$$
\int_{r_{s_{s t}}+\eta_{s}}^{R_{s}} \overline{p_{s_{p}} u_{s_{p}}} 2 \pi r \mathrm{~d} r=\int_{r_{0_{s t}}+\eta_{0}}^{1} \overline{p_{0_{p}}\left(u_{0_{s t}}+u_{0_{p}}\right)} 2 \pi r \mathrm{~d} r
$$

donde la línea sobre las variables indica valor promedio, $p_{s_{p}}, u_{s_{p}} \mathrm{y} \eta_{s}$ son las perturbaciones en la presión, la velocidad axial y el radio del núcleo de aire en la cámara, $p_{0_{p}}, u_{0_{p}}$ y $\eta_{0}$ son los análogos cerca del orificio de salida, $R_{s}$ es el radio de la pared en la cámara, $r_{s_{s t}}$ y $r_{0_{s t}}$ son los radios del núcleo de aire en régimen estacionario en la cámara y cerca del orificio de salida, y $u_{0_{s t}}$ es la velocidad axial en régimen estacionario cerca de dicho orificio. Desarrollando las integrales y eliminando los términos que oscilan:

$$
\begin{aligned}
\int_{r_{s t}}^{R_{s}} \overline{p_{s_{p}} u_{s_{p}}} 2 \pi r \mathrm{~d} r=\int_{r_{0_{s t}}}^{1} \overline{p_{0_{p}} u_{0_{p}}} 2 \pi r \mathrm{~d} r+\int_{r_{0_{s t}}+\eta_{0}}^{r_{0_{s t}}} \overline{p_{0_{p}} u_{0_{s t}}} 2 \pi r \mathrm{~d} r= \\
\int_{r_{0_{s t}}}^{1} \overline{p_{0_{p}} u_{0_{p}}} 2 \pi r \mathrm{~d} r-2 \pi \overline{\left(p_{0_{p}} u_{0_{s t}} r\right)_{r=r_{0}} \eta_{0}}
\end{aligned}
$$


B. Propagación de ondas en la superficie del núcleo de aire a través de una región de 134 transición compacta

Estas integrales se pueden expresar de la siguiente manera:

$$
\begin{aligned}
& \int_{r_{s_{s t}}}^{R_{s}} \overline{p_{s_{p}} u_{s_{p}}} 2 \pi r \mathrm{~d} r=-\int_{r_{s_{s t}}}^{R_{s}} \overline{\frac{\partial \varphi_{s_{p}}}{\partial t} \frac{\partial \varphi_{s_{p}}}{\partial x}} 2 \pi r \mathrm{~d} r \\
& \int_{r_{0_{s t}}}^{1} \overline{p_{0_{p}} u_{0_{p}}} 2 \pi r \mathrm{~d} r=-\int_{r_{0_{s t}}}^{1}\left[\overline{\frac{\partial \varphi_{0_{p}}}{\partial t} \frac{\partial \varphi_{0_{p}}}{\partial x}}+u_{0_{s t}}\left(\overline{\frac{\partial \varphi_{0_{p}}}{\partial x}}\right)^{2}\right] 2 \pi r \mathrm{~d} r \\
& -2 \pi \overline{\left(p_{0_{p}} u_{0_{s t}} r\right)_{r=r_{0_{s t}}} \eta_{0}}=2 \pi u_{0_{s t}} r_{0_{s t}} \overline{\frac{\partial \varphi_{0_{p}}}{\partial t} \eta_{0}}+2 \pi u_{0_{s t}}^{2} r_{0_{s t}} \frac{\overline{\partial \varphi_{0_{p}}}}{\partial x} \eta_{0}
\end{aligned}
$$

donde $\varphi_{s_{p}}$ y $\varphi_{0_{p}}$ son las perturbaciones en el potencial de velocidades en la cámara y cerca del orificio de salida, respectivamente.

Las perturbaciones de los potenciales de velocidad en la cámara y en la región en torno al orificio, así como la deformación de la superficie líquida en esta última zona, vienen dados por:

$$
\begin{gathered}
\varphi_{s_{p}}=a_{s} F_{s}(r) e^{-i k_{s} x+i \omega t}+a_{s}^{*} F_{s}(r) e^{i k_{s} x-i \omega t}+d_{s} F_{s}(r) e^{i k_{s} x+i \omega t}+d_{s}^{*} F_{s}(r) e^{-i k_{s} x-i \omega t} \\
\varphi_{0_{p}}=a_{0} F_{0} e^{-i k_{0} x+i \omega t}+a_{0}^{*} F_{0} e^{i k_{0} x-i \omega t} \\
\eta_{0}=i\left(\omega-U_{0} k_{0}\right) r_{0}^{3} a_{0} F_{0} e^{-i k_{0} x+i \omega t}-i\left(\omega-U_{0} k_{0}\right) r_{0}^{3} a_{0}^{*} F_{0} e^{i k_{0} x-i \omega t}
\end{gathered}
$$

donde $a_{s}^{*}, d_{s}^{*}$ y $a_{0}^{*}$ son los complejos conjugados de $a_{s}, d_{s}$ y $a_{0}$, respectivamente, y $F_{s}(r)$ y $F_{0}$ se definen de la siguiente manera:

$$
F_{s}(r)=\left(\frac{K_{0}\left(k_{s} r\right)}{K_{0}^{\prime}\left(k_{s} R_{s}\right)}-\frac{I_{0}\left(k_{s} r\right)}{I_{0}^{\prime}\left(k_{s} R_{s}\right)}\right) \quad ; \quad F_{0}=-\frac{2}{k_{0}}
$$

Así pues, se obtiene:

$$
\begin{gathered}
\frac{\partial \varphi_{s_{p}}}{\partial t} \frac{\partial \varphi_{s_{p}}}{\partial x}=\omega k_{s} a_{s}^{2} F_{s}^{2}(r) e^{-i 2 k_{s} x+i 2 \omega t}-2 \omega k_{s}\left|a_{s}\right|^{2} F_{s}^{2}(r)+\omega k_{s} a_{s}^{* 2} F_{s}^{2}(r) e^{i 2 k_{s} x-i 2 \omega t} \\
-\omega k_{s} d_{s}^{2} F_{s}^{2}(r) e^{i 2 k_{s} x+i 2 \omega t}+2 \omega k_{s}\left|d_{s}\right|^{2} F_{s}^{2}(r)-\omega k_{s} d_{s}^{* 2} F_{s}^{2}(r) e^{-i 2 k_{s} x-i 2 \omega t} \\
\frac{\partial \varphi_{0_{p}}}{\partial t} \frac{\partial \varphi_{0_{p}}}{\partial x}=\omega k_{0} a_{0}^{2} F_{0}^{2} e^{-i 2 k_{0} x+i 2 \omega t}-2 \omega k_{0}\left|a_{0}\right|^{2} F_{0}^{2}+\omega k_{0} a_{0}^{* 2} F_{0}^{2} e^{i 2 k_{0} x-i 2 \omega t} \\
\left(\frac{\partial \varphi_{0_{p}}}{\partial x}\right)^{2}=-k_{0}^{2} a_{0}^{2} F_{0}^{2} e^{-i 2 k_{0} x+i 2 \omega t}+2 k_{0}^{2}\left|a_{0}\right|^{2} F_{0}^{2}-k_{0}^{2} a_{0}^{* 2} F_{0}^{2} e^{i 2 k_{0} x-i 2 \omega t} \\
\frac{\partial \varphi_{0_{p}}}{\partial t} \eta_{0}=-\omega\left(\omega-u_{0_{s t}} k_{0}\right) r_{0_{s t}}^{3} a_{0}^{2} F_{0}^{2} e^{-i 2 k_{0} x+i 2 \omega t} \\
+2 \omega\left(\omega-u_{0_{s t}} k_{0}\right) r_{0_{s t}}^{3}\left|a_{0}\right|^{2} F_{0}^{2}-\omega\left(\omega-u_{0_{s t}} k_{0}\right) r_{0_{s t}}^{3} a_{0}^{* 2} F_{0}^{2} e^{i 2 k_{0} x-i 2 \omega t}
\end{gathered}
$$




$$
\begin{aligned}
\frac{\partial \varphi_{0_{p}}}{\partial x} \eta_{0}=k_{0}\left(\omega-u_{0_{s t}} k_{0}\right) r_{0_{s t}}^{3} a_{0}^{2} F_{0}^{2} e^{-i 2 k_{0} x+i 2 \omega t} & \\
& -2 k_{0}\left(\omega-u_{0_{s t}} k_{0}\right) r_{0_{s t}}^{3}\left|a_{0}\right|^{2} F_{0}^{2}+k_{0}\left(\omega-u_{0_{s t}} k_{0}\right) r_{0_{s t}}^{3} a_{0}^{* 2} F_{0}^{2} e^{i 2 k_{0} x-i 2 \omega t}
\end{aligned}
$$

Para el promedio, se eliminan los términos que oscilan:

$$
\begin{aligned}
& \overline{\frac{\partial \varphi_{s_{p}}}{\partial t} \frac{\partial \varphi_{s_{p}}}{\partial x}}=-2 \omega k_{s}\left(\left|a_{s}\right|^{2}-\left|d_{s}\right|^{2}\right) F_{s}^{2}(r) \\
& \overline{\frac{\partial \varphi_{0_{p}}}{\partial t} \frac{\partial \varphi_{0_{p}}}{\partial x}}=-2 \omega k_{0}\left|a_{0}\right|^{2} F_{0}^{2} \\
& \left(\overline{\frac{\partial \varphi_{0_{p}}}{\partial x}}\right)^{2}=2 k_{0}^{2}\left|a_{0}\right|^{2} F_{0}^{2} \\
& \overline{\frac{\partial \varphi_{0_{p}}}{\partial t} \eta_{0}}=2 \omega\left(\omega-u_{0_{s t}} k_{0}\right) r_{0_{s t}}^{3}\left|a_{0}\right|^{2} F_{0}^{2} \\
& \overline{\frac{\partial \varphi_{0_{p}}}{\partial x} \eta_{0}}=-2 k_{0}\left(\omega-u_{0_{s t}} k_{0}\right) r_{0_{s t}}^{3}\left|a_{0}\right|^{2} F_{0}^{2}
\end{aligned}
$$

Con estos promedios la expresión (2.136) da lugar a:

$4 \pi \omega k_{s}\left(\left|a_{s}\right|^{2}-\left|d_{s}\right|^{2}\right) \int_{r_{s_{s t}}}^{R_{s}} F_{s}^{2}(r) r \mathrm{~d} r=\frac{8 \pi\left(\omega-u_{0_{s t}} k_{0}\right)\left|a_{0}\right|^{2}}{k_{0}}\left(1-r_{0_{s t}}^{2}+\frac{2 r_{0_{s t}}^{4} u_{0_{s t}}\left(\omega-u_{0_{s t}} k_{0}\right)}{k_{0}}\right)$

de donde obtenemos la disipación:

$$
1-\frac{\left|d_{s}\right|^{2}}{\left|a_{s}\right|^{2}}=\frac{2\left(\omega-u_{0 s t} k_{0}\right)}{\omega k_{s} k_{0}} \frac{\left|a_{0}\right|^{2}}{\left|a_{s}\right|^{2}}\left(1-r_{0_{s t}}^{2}+\frac{2 r_{0_{s t}}^{4} u_{0_{s t}}\left(\omega-u_{0_{s t}} k_{0}\right)}{k_{0}}\right)\left(\int_{r_{s_{s t}}}^{R_{s}} F_{s}^{2}(r) r \mathrm{~d} r\right)^{-1}
$$


B. Propagación de ondas en la superficie del núcleo de aire a través de una región de 136 transición compacta 


\section{Apéndice $\mathrm{C}$}

\section{Propagación de ondas en la} superficie del núcleo de aire en una cámara de sección lentamente variable

En este Apéndice se incluye el desarrollo del análisis de propagación de ondas en la superficie del núcleo de aire en una cámara de sección lentamente variable presentado en la Sección 2.6. El problema a resolver está gobernado por las siguientes ecuaciones:

$$
\begin{gathered}
\frac{\partial^{2} \varphi}{\partial x^{2}}+\frac{1}{r} \frac{\partial}{\partial r}\left(r \frac{\partial \varphi}{\partial r}\right)=0 \\
\frac{\partial r_{a c}}{\partial t}+\frac{\partial \varphi}{\partial x} \frac{\partial r_{a c}}{\partial x}-\frac{\partial \varphi}{\partial r}=0 \quad \text { en } \quad r=r_{a c} \\
\frac{\partial \varphi}{\partial t}+\frac{1}{2}\left(\frac{\partial \varphi}{\partial x}\right)^{2}+\frac{1}{2}\left(\frac{\partial \varphi}{\partial r}\right)^{2}+\frac{\Gamma^{2}}{8 \pi^{2} r_{a c}^{2}}+\frac{p_{a}}{\rho}=C(t) \quad \text { en } \quad r=r_{a c} \\
\frac{\partial \varphi}{\partial r}-\frac{\mathrm{d} R_{w}}{\mathrm{~d} x} \frac{\partial \varphi}{\partial x}=0 \quad \text { en } \quad r=R_{w}
\end{gathered}
$$

donde $\varphi, \rho, \Gamma, p_{a}, R_{w}$ y $r_{a c}$ son el potencial de velocidades y la densidad del líquido, la circulación, la presión del ambiente y los radios de la pared interna de la cámara y del núcleo de aire, respectivamente. Podemos admitir que la variación $\Delta C(t)$ de la constante $C(t)$ de la ecuación de Euler-Bernoulli respecto a su valor en ausencia de perturbaciones se considera incluida en el potencial de velocidades, redefiniendo el mismo añadiéndole $\int_{0}^{t} \Delta C(t) \mathrm{d} t$. Así pues:

$$
C=\frac{1}{2} u_{s}^{2}+\frac{\Gamma^{2}}{8 \pi^{2} r_{s}^{2}}+\frac{p_{a}}{\rho}
$$


donde $u_{s}, R_{s}$ y $r_{s}$ son la velocidad axial del líquido, el radio de la pared de la cámara y el radio del núcleo de aire aguas arriba de la región donde la sección empieza a variar el radio de la pared de la cámara. El caudal será $Q=\pi u_{s}\left(R_{s}^{2}-r_{s}^{2}\right)$. Escalando la distancia radial con $R_{s}$, la distancia axial con $\beta R_{s}$, siendo $\beta \gg 1$, el potencial de velocidad con $\Gamma /(2 \pi)$ y el tiempo con $2 \pi R_{s}^{2} / \Gamma$, las ecuaciones (C.1) - (C.4) quedan, en variables adimensionales:

$$
\begin{gathered}
\frac{1}{\beta^{2}} \frac{\partial^{2} \varphi}{\partial x^{2}}+\frac{1}{r} \frac{\partial}{\partial r}\left(r \frac{\partial \varphi}{\partial r}\right)=0 \\
\frac{\partial r_{a c}}{\partial t}-\frac{\partial \varphi}{\partial r}+\frac{1}{\beta^{2}} \frac{\partial \varphi}{\partial x} \frac{\partial r_{a c}}{\partial x}=0 \quad \text { en } \quad r=r_{a c} \\
\frac{\partial \varphi}{\partial t}+\frac{1}{2}\left(\frac{\partial \varphi}{\partial r}\right)^{2}+\frac{1}{2 r_{a c}^{2}}+\frac{1}{2 \beta^{2}}\left(\frac{\partial \varphi}{\partial x}\right)^{2}=\frac{1}{2} u_{s}^{2}+\frac{1}{2 r_{s}^{2}} \quad \text { en } \quad r=r_{a c} \\
\frac{\partial \varphi}{\partial r}-\frac{1}{\beta^{2}} \frac{\mathrm{d} R_{w}}{\mathrm{~d} x} \frac{\partial \varphi}{\partial x}=0 \quad \text { en } \quad r=R_{w}
\end{gathered}
$$

\section{C.1 Solución estacionaria}

Llamamos $\varphi_{s t}$ y $r_{a c_{s t}}$ a la solución estacionaria del potencial de velocidad y del radio del núcleo de aire, respectivamente. Las ecuaciones (C.5) - (C.8) en régimen estacionario quedan:

$$
\begin{gathered}
\frac{1}{\beta^{2}} \frac{\partial^{2} \varphi_{s t}}{\partial x^{2}}+\frac{1}{r} \frac{\partial}{\partial r}\left(r \frac{\partial \varphi_{s t}}{\partial r}\right)=0 \\
-\frac{\partial \varphi_{s t}}{\partial r}+\frac{1}{\beta^{2}} \frac{\partial \varphi_{s t}}{\partial x} \frac{\mathrm{d} r_{a c_{s t}}}{\mathrm{~d} x}=0 \quad \text { en } \quad r=r_{a c_{s t}} \\
\frac{1}{2}\left(\frac{\partial \varphi_{s t}}{\partial r}\right)^{2}+\frac{1}{2 r_{a c_{s t}}^{2}}+\frac{1}{2 \beta^{2}}\left(\frac{\partial \varphi_{s t}}{\partial x}\right)^{2}=\frac{1}{2} u_{s}^{2}+\frac{1}{2 r_{s}^{2}} \quad \text { en } \quad r=r_{a c_{s t}} \\
\frac{\partial \varphi_{s t}}{\partial r}-\frac{1}{\beta^{2}} \frac{\mathrm{d} R_{w}}{\mathrm{~d} x} \frac{\partial \varphi_{s t}}{\partial x}=0 \quad \text { en } \quad r=R_{w}
\end{gathered}
$$

Desarrollando $\varphi_{s t}$ y $r_{a c_{s t}}$ en potencias de $\beta^{-1}$ :

$$
\varphi_{s t}=\beta\left(\varphi_{s t 0}+\frac{1}{\beta^{2}} \varphi_{s t 2}+\ldots\right) \quad ; \quad r_{a c_{s t}}=r_{a c_{s t 0}}+\frac{1}{\beta^{2}} r_{a c_{s t 2}}+\ldots
$$

Sustituyendo (C.13) en (C.9) y tomando los términos de mayor orden:

$$
\frac{\partial}{\partial r}\left(r \frac{\partial \varphi_{s t 0}}{\partial r}\right)=0
$$

cuya solución es:

$$
\varphi_{s t 0}=f_{0}(x)+g_{0}(x) \ln r
$$


De la condición de contorno (C.12) se obtiene:

$$
\frac{g_{0}(x)}{R_{w}}=0
$$

luego $g_{0}(x)=0, \mathrm{y} \varphi_{s t 0}=f_{0}(x)$. Tomando los términos de siguiente orden:

$$
\frac{\partial^{2} \varphi_{s t 0}}{\partial x^{2}}+\frac{1}{r} \frac{\partial}{\partial r}\left(r \frac{\partial \varphi_{s t 2}}{\partial r}\right)=0
$$

de donde se obtiene:

$$
\varphi_{s t 2}=-\frac{r^{2}}{4} \frac{\mathrm{d}^{2} f_{0}(x)}{\mathrm{d} x^{2}}+f_{2}(x)+g_{2}(x) \ln r
$$

y la solución estacionaria del potencial de velocidades queda:

$$
\varphi_{s t}=\beta f_{0}(x)+\frac{1}{\beta}\left(-\frac{r^{2}}{4} \frac{\mathrm{d}^{2} f_{0}(x)}{\mathrm{d} x^{2}}+f_{2}(x)+g_{2}(x) \ln r\right)+\ldots
$$

Introduciendo (C.14) en (C.10) - (C.12), y tomando los términos de mayor orden:

$$
\begin{gathered}
-\left(-\frac{r_{a c_{s t 0}}}{2} \frac{\mathrm{d}^{2} f_{0}}{\mathrm{~d} x^{2}}+\frac{g_{2}}{r_{a c_{s t 0}}}\right)+\frac{\mathrm{d} f_{0}}{\mathrm{~d} x} \frac{\mathrm{d} r_{a c_{s t 0}}}{\mathrm{~d} x}=0 \\
\left(\frac{\mathrm{d} f_{0}}{\mathrm{~d} x}\right)^{2}+\frac{1}{r_{a c_{s t 0}}^{2}}=u_{s}^{2}+\frac{1}{r_{s}^{2}} \\
\left(-\frac{R_{w}}{2} \frac{\mathrm{d}^{2} f_{0}}{\mathrm{~d} x^{2}}+\frac{g_{2}}{R_{w}}\right)-\frac{\mathrm{d} R_{w}}{\mathrm{~d} x} \frac{\mathrm{d} f_{0}}{\mathrm{~d} x}=0
\end{gathered}
$$

Multiplicando (C.15) por $r_{a c_{s t 0}}$ y (C.17) por $R_{w}$, y sumando ambas expresiones:

$$
\frac{\mathrm{d}}{\mathrm{d} x}\left[\frac{\mathrm{d} f_{0}}{\mathrm{~d} x}\left(R_{w}^{2}-r_{a c_{s t 0}}^{2}\right)\right]=0
$$

Teniendo en cuenta que $\mathrm{d} f_{0} / \mathrm{d} x$ es la componente axial de la velocidad, la ecuación (C.18) se traduce en que el caudal no varía a lo largo de la coordenada axial. Así pues, la relación (C.18) se puede expresar de la siguiente manera:

$$
\frac{\mathrm{d} f_{0}}{\mathrm{~d} x}\left(R_{w}^{2}-r_{a c_{s t 0}}^{2}\right)=\frac{Q}{\pi}
$$

Las expresiones (C.16) y (C.19) forman un sistema de dos ecuaciones con dos incógnitas, la velocidad axial $\mathrm{d} f_{0} / \mathrm{d} x$ y el radio del núcleo de aire $r_{c_{s t 0}}$. Combinando ambas se obtiene la siguiente expresión para $r_{a c_{s t 0}}$ :

$$
u_{s}^{2}\left(\frac{1-r_{s}^{2}}{R_{w}^{2}-r_{a c_{s t 0}}^{2}}\right)^{2}+\frac{1}{r_{a c_{s t 0}}^{2}}=u_{s}^{2}+\frac{1}{r_{s}^{2}}
$$


C. Propagación de ondas en la superficie del núcleo de aire en una cámara de sección 140

lentamente variable

\section{C.2 Problema dependiente del tiempo}

Introducimos pequeñas perturbaciones en el radio del núcleo de aire y en el potencial de velocidades, que designaremos $\eta$ y $\varphi_{p}$, respectivamente, tales que $\eta \ll r_{a c_{s t}} \mathrm{y} \varphi_{p} \ll \varphi_{s t}$, esto es:

$$
\varphi=\varphi_{s t}+\varphi_{p} \quad \mathrm{y} \quad r_{a c}=r_{a c_{s t}}+\eta
$$

Sustituyendo (C.21) en (C.5) - (C.8), y linealizando:

$$
\begin{gathered}
\frac{1}{\beta^{2}} \frac{\partial^{2} \varphi_{p}}{\partial x^{2}}+\frac{1}{r} \frac{\partial}{\partial r}\left(r \frac{\partial \varphi_{p}}{\partial r}\right)=0 \\
\frac{\partial \eta}{\partial t}-\frac{\partial \varphi_{p}}{\partial r}-\eta \frac{\partial^{2} \varphi_{s t}}{\partial r^{2}}+\frac{1}{\beta^{2}}\left(\frac{\partial \varphi_{s t}}{\partial x} \frac{\partial \eta}{\partial x}+\frac{\partial \varphi_{p}}{\partial x} \frac{\mathrm{d} r_{a c_{s t}}}{\mathrm{~d} x}\right)=0 \quad \text { en } \quad r=r_{a c_{s t}} \\
\frac{\partial \varphi_{p}}{\partial t}+\frac{\partial \varphi_{s t}}{\partial r} \frac{\partial \varphi_{p}}{\partial r}+\eta \frac{\partial^{2} \varphi_{s t}}{\partial r^{2}} \frac{\partial \varphi_{s t}}{\partial r}+\frac{1}{\beta^{2}} \frac{\partial \varphi_{s t}}{\partial x} \frac{\partial \varphi_{p}}{\partial x}-\frac{\eta}{r_{a c_{s t}}^{3}}=0 \quad \text { en } \quad r=r_{a c_{s t 0}} \\
\frac{\partial \varphi_{p}}{\partial r}-\frac{1}{\beta^{2}} \frac{\mathrm{d} R_{w}}{\mathrm{~d} x} \frac{\partial \varphi_{p}}{\partial x}=0 \quad \text { en } \quad r=R_{w}
\end{gathered}
$$

Como los coeficientes de las ecuaciones del problema lineal son independientes del tiempo, podemos buscar soluciones de la forma:

$$
\varphi_{p}=\hat{\varphi}_{p}(x, r) e^{i \omega t}+c c \quad \text { y } \quad \eta=\hat{\eta}(x) e^{i \omega t}+c c
$$

donde $c c$ indica complejo conjugado. Sustituyendo (C.26) en (C.22) - (C.25):

$$
\begin{gathered}
\frac{1}{\beta^{2}} \frac{\partial^{2} \hat{\varphi}_{p}}{\partial x^{2}}+\frac{1}{r} \frac{\partial}{\partial r}\left(r \frac{\partial \hat{\varphi}_{p}}{\partial r}\right)=0 \\
i \omega \hat{\eta}-\frac{\partial \hat{\varphi}_{p}}{\partial r}-\hat{\eta} \frac{\partial^{2} \varphi_{s t}}{\partial r^{2}}+\frac{1}{\beta^{2}}\left(\frac{\partial \varphi_{s t}}{\partial x} \frac{\mathrm{d} \hat{\eta}}{\mathrm{d} x}+\frac{\partial \hat{\varphi}_{p}}{\partial x} \frac{\mathrm{d} r_{a c_{s t}}}{\mathrm{~d} x}\right)=0 \quad \text { en } \quad r=r_{a c_{s t 0}} \\
i \omega \hat{\varphi}_{p}+\frac{\partial \varphi_{s t}}{\partial r} \frac{\partial \hat{\varphi}_{p}}{\partial r}+\hat{\eta} \frac{\partial^{2} \varphi_{s t}}{\partial r^{2}} \frac{\partial \varphi_{s t}}{\partial r}+\frac{1}{\beta^{2}} \frac{\partial \varphi_{s t}}{\partial x} \frac{\partial \hat{\varphi}_{p}}{\partial x}-\frac{\hat{\eta}}{r_{a c_{s t}}^{3}}=0 \quad \text { en } \quad r=r_{a c_{s t 0}} \\
\frac{\partial \hat{\varphi}_{p}}{\partial r}-\frac{1}{\beta^{2}} \frac{\mathrm{d} R_{w}}{\mathrm{~d} x} \frac{\partial \hat{\varphi}_{p}}{\partial x}=0 \quad \text { en } \quad r=R_{w}
\end{gathered}
$$

Buscamos soluciones de este problema con el método WKB. Para ello introducimos tres nuevas funciones desconocidas, $A(x, r), D(x)$ y $s(x)$, y expresamos $\hat{\varphi}_{p}$ y $\hat{\eta}$ en términos de estas funciones mediante las relaciones:

$$
\hat{\varphi}_{p}=A(x, r) e^{i \beta s(x)} \quad \text { y } \quad \hat{\eta}=D(x) e^{i \beta s(x)}
$$


Insertando (C.31) en (C.27) - (C.30) se llega a las siguientes expresiones:

$$
\begin{gathered}
{\left[\frac{1}{r} \frac{\partial}{\partial r}\left(r \frac{\partial A}{\partial r}\right)-A\left(\frac{\mathrm{d} s}{\mathrm{~d} x}\right)^{2}\right]+\frac{1}{\beta} i\left(2 \frac{\partial A}{\partial x} \frac{\mathrm{d} s}{\mathrm{~d} x}+A \frac{\mathrm{d}^{2} s}{\mathrm{~d} x^{2}}\right)+\frac{1}{\beta^{2}} \frac{\partial^{2} A}{\partial x^{2}}=0 \quad \text { (C.32) }} \\
i D\left(\omega+\frac{\mathrm{d} s}{\mathrm{~d} x} \frac{\mathrm{d} f_{0}}{\mathrm{~d} x}\right)-\frac{\partial A}{\partial r}+\frac{1}{\beta}\left[i A \frac{\mathrm{d} s}{\mathrm{~d} x} \frac{\mathrm{d} r_{a c_{s t 0}}}{\mathrm{~d} x}+\frac{\mathrm{d} f_{0}}{\mathrm{~d} x} \frac{\mathrm{d} D}{\mathrm{~d} x}+D\left(\frac{1}{r_{a c_{s t 0}}} \frac{\mathrm{d} r_{a c_{s t 0} 0}}{\mathrm{~d} x} \frac{\mathrm{d} f_{0}}{\mathrm{~d} x}+\frac{\mathrm{d}^{2} f_{0}}{\mathrm{~d} x^{2}}\right)\right] \\
+O\left(\beta^{-2}\right)=0 \quad \text { en } \quad r=r_{a c_{s t 0} 0} \quad \text { (C.33) } \\
i A\left(\omega+\frac{\mathrm{d} s}{\mathrm{~d} x} \frac{\mathrm{d} f_{0}}{\mathrm{~d} x}\right)-\frac{D}{r_{a c_{s t 0}}^{3}}+\frac{1}{\beta}\left(\frac{\mathrm{d} f_{0}}{\mathrm{~d} x} \frac{\left.\mathrm{d} r_{a c_{s t 0}} \frac{\partial A}{\partial r}+\frac{\mathrm{d} f_{0}}{\mathrm{~d} x} \frac{\partial A}{\partial x}\right)}{+O\left(\beta^{-2}\right)=0 \quad \text { en } \quad r=r_{a c_{s t 0}} \quad \text { (C.34) }}\right. \\
\frac{\partial A}{\partial r}-\frac{1}{\beta} i A \frac{\mathrm{d} R_{w}}{\mathrm{~d} x} \frac{\mathrm{d} s}{\mathrm{~d} x}+O\left(\beta^{-2}\right)=0 \quad \text { en } \quad r=R_{w}
\end{gathered}
$$

Para resolver las ecuaciones (C.32) - (C.35) asumiremos que, para valores grandes de $\beta$, las funciones $A(x, r)$ y $D(x)$ tienen unos desarrollos asintóticos de la siguiente forma:

$$
A \sim A_{0}(x, r)+\sum_{n=1}^{\infty} \frac{A_{n}(x, r)}{(i \beta)^{n}} \quad \text { y } \quad D \sim D_{0}(x)+\sum_{n=1}^{\infty} \frac{D_{n}(x)}{(i \beta)^{n}}
$$

Introduciendo los desarrollos dados en (C.36) en (C.32) - (C.35), agrupando en potencias de $\beta$, y llamando $k=|\mathrm{d} s / \mathrm{d} x|$ y $u_{s t 0}=\mathrm{d} f_{0} / \mathrm{d} x$ :

$$
\begin{aligned}
& \frac{1}{r} \frac{\partial}{\partial r}\left(r \frac{\partial A_{0}}{\partial r}\right)-k^{2} A_{0}+\frac{1}{\beta} i\left[2 k \frac{\partial A_{0}}{\partial x}+A_{0} \frac{\mathrm{d} k}{\mathrm{~d} x}-\frac{1}{r} \frac{\partial}{\partial r}\left(r \frac{\partial A_{1}}{\partial r}\right)+k^{2} A_{1}\right] \\
& +O\left(\beta^{-2}\right)=0 \\
& i D_{0}\left(\omega+u_{s t 0} k\right)-\frac{\partial A_{0}}{\partial r} \\
& +\frac{1}{\beta}\left[i A_{0} k \frac{\mathrm{d} r_{a c_{s t 0}}}{\mathrm{~d} x}+u_{s t 0} \frac{\mathrm{d} D_{0}}{\mathrm{~d} x}+D_{0}\left(\frac{u_{s t 0}}{r_{a c_{s t 0}}} \frac{\mathrm{d} r_{a c_{s t 0}}}{\mathrm{~d} x}+\frac{\mathrm{d} u_{s t 0}}{\mathrm{~d} x}\right)+D_{1}\left(\omega+u_{s t 0} k\right)+i \frac{\partial A_{1}}{\partial r}\right] \\
& +O\left(\beta^{-2}\right)=0 \quad \text { en } \quad r=r_{a c_{s t 0}} \\
& i A_{0}\left(\omega+u_{s t 0} k\right)-\frac{D_{0}}{r_{a c_{s t 0}}^{3}}+\frac{1}{\beta}\left[u_{s t 0} \frac{\mathrm{d} r_{a c_{s t 0}}}{\mathrm{~d} x} \frac{\partial A_{0}}{\partial r}+u_{s t 0} \frac{\partial A_{0}}{\partial x}+A_{1}\left(\omega+u_{s t 0} k\right)+i \frac{D_{1}}{r_{a c_{s t 0}}^{3}}\right] \\
& +O\left(\beta^{-2}\right)=0 \quad \text { en } \quad r=r_{a c_{s t 0}}
\end{aligned}
$$


C. Propagación de ondas en la superficie del núcleo de aire en una cámara de sección 142

$$
\frac{\partial A_{0}}{\partial r}-\frac{1}{\beta} i\left(A_{0} k \frac{\mathrm{d} R_{w}}{\mathrm{~d} x}+\frac{\partial A_{1}}{\partial r}\right)+O\left(\beta^{-2}\right)=0 \quad \text { en } \quad r=R_{w}
$$

Tomando los términos de mayor orden de (C.37) - (C.40):

$$
\begin{gathered}
\frac{1}{r} \frac{\partial}{\partial r}\left(r \frac{\partial A_{0}}{\partial r}\right)-k^{2} A_{0}=0 \\
i D_{0}\left(\omega+u_{s t 0} k\right)-\frac{\partial A_{0}}{\partial r}=0 \quad \text { en } \quad r=r_{a c_{s t 0}} \\
i A_{0}\left(\omega+u_{s t 0} k\right)-\frac{D_{0}}{r_{a c_{s t 0}}^{3}}=0 \quad \text { en } \quad r=r_{a c_{s t 0}} \\
\frac{\partial A_{0}}{\partial r}=0 \quad \text { en } \quad r=R_{w}
\end{gathered}
$$

La solución de la ecuación (C.41), teniendo en cuenta la condición de contorno (C.44), es de la forma:

$$
A_{0}=a(x) F(x, r)
$$

con

$$
F(x, r)=\frac{K_{0}(k r)}{K_{0}^{\prime}\left(k R_{w}\right)}-\frac{I_{0}(k r)}{I_{0}^{\prime}\left(k R_{w}\right)}
$$

Despejando $D_{0}$ de (C.43):

$$
D_{0}=i r_{a c_{s t 0}}^{3} A_{0}\left(x, r_{c_{s t 0}}\right)\left(\omega+u_{s t 0} k\right)
$$

Sustituyendo (C.47) en (C.42):

$$
A_{0}\left(\omega+u_{s t 0} k\right)^{2}+\frac{1}{r_{a c_{s t 0}}^{3}} \frac{\partial A_{0}}{\partial r}=0 \quad \text { en } \quad r=r_{a c}
$$

Introduciendo (C.45) en (C.48) se obtiene la siguiente relación entre $\omega$ y $k$ :

$$
\left(\omega+u_{s t 0} k\right)^{2}=-\frac{k}{r_{a c_{s t 0}}^{3}} \frac{K_{0}^{\prime}\left(k r_{a c_{s t 0}}\right) I_{0}^{\prime}\left(k R_{w}\right)-K_{0}^{\prime}\left(k R_{w}\right) I_{0}^{\prime}\left(k r_{a c_{s t 0}}\right)}{K_{0}\left(k r_{a c_{s t 0}}\right) I_{0}^{\prime}\left(k R_{w}\right)-K_{0}^{\prime}\left(k R_{w}\right) I_{0}\left(k r_{a c_{s t 0}}\right)}
$$

Tomando ahora los términos de orden $\beta^{-1}$ de (C.37) - (C.40):

$$
\begin{gathered}
\frac{1}{r} \frac{\partial}{\partial r}\left(r \frac{\partial A_{1}}{\partial r}\right)-k^{2} A_{1}=2 k \frac{\partial A_{0}}{\partial x}+A_{0} \frac{\mathrm{d} k}{\mathrm{~d} x} \\
D_{1}\left(\omega+u_{s t 0} k\right)+i \frac{\partial A_{1}}{\partial r}+i k A_{0} \frac{\mathrm{d} r_{a c_{s t 0}}}{\mathrm{~d} x}+u_{s t 0} \frac{\mathrm{d} D_{0}}{\mathrm{~d} x} \\
+D_{0}\left(\frac{u_{s t 0}}{r_{a c_{s t 0}}} \frac{\mathrm{d} r_{a c_{s t 0}}}{\mathrm{~d} x}+\frac{\mathrm{d} u_{s t 0}}{\mathrm{~d} x}\right)=0 \quad \text { en } \quad r=r_{a c_{s t 0}}
\end{gathered}
$$




$$
\begin{gathered}
A_{1}\left(\omega+u_{s t 0} k\right)+i \frac{D_{1}}{r_{a c_{s t 0}}^{3}}+u_{s t 0} \frac{\mathrm{d} r_{a c_{s t 0}}}{\mathrm{~d} x} \frac{\partial A_{0}}{\partial r}+u_{s t 0} \frac{\partial A_{0}}{\partial x}=0 \quad \text { en } \quad r=r_{a c_{s t 0}} \\
A_{0} k \frac{\mathrm{d} R_{w}}{\mathrm{~d} x}+\frac{\partial A_{1}}{\partial r}=0 \quad \text { en } \quad r=R_{w}
\end{gathered}
$$

Eliminando $D_{1}$ entre (C.51) y (C.52), y sustituyendo el valor de $D_{0}$ dado por (C.47):

$$
\begin{aligned}
A_{1}\left(\omega+u_{s t 0} k\right)^{2}+ & \frac{1}{r_{a c_{s t 0}}^{3}} \frac{\partial A_{1}}{\partial r}+A_{0}\left(\frac{k}{r_{a c_{s t 0}}^{3}} \frac{\mathrm{d} r_{a c_{s t 0}}}{\mathrm{~d} x}+u_{s t 0} \frac{\mathrm{d}\left(u_{s t 0} k\right)}{\mathrm{d} x}\right) \\
+ & \left(2 \frac{\mathrm{d} r_{a c_{s t 0}}}{\mathrm{~d} x} \frac{\partial A_{0}}{\partial r}+2 \frac{\partial A_{0}}{\partial x}+\frac{3 A_{0}}{r_{a c_{s t 0}}} \frac{\mathrm{d} r_{a c_{s t 0}}}{\mathrm{~d} x}\right) u_{s t 0}\left(\omega+u_{s t 0} k\right) \\
& +A_{0}\left(\omega+u_{s t 0} k\right)\left(\frac{u_{s t 0}}{r_{a c_{s t 0}}} \frac{\mathrm{d} r_{a c_{s t 0}}}{\mathrm{~d} x}+\frac{\mathrm{d} u_{s t 0}}{\mathrm{~d} x}\right)=0 \quad \text { en } \quad r=r_{a c_{s t 0}}
\end{aligned}
$$

Para resolver el problema formado por la ecuación (C.50) y las condiciones de contorno (C.53) y (C.54), introducimos una nueva función desconocida, $B_{1}(x, r)$, tal que:

$$
A_{1}(x, r)=F(x, r) B_{1}(x, r)
$$

Sustituyendo (C.55) en (C.50), (C.54) y (C.53), teniendo en cuenta que $F$ satisface (C.41), y usando (C.42) - (C.44), se obtiene:

$$
\begin{gathered}
\frac{1}{r F} \frac{\partial}{\partial r}\left(r F^{2} \frac{\partial B_{1}}{\partial r}\right)=2 k \frac{\partial A_{0}}{\partial x}+A_{0} \frac{\mathrm{d} k}{\mathrm{~d} x} \\
\frac{F}{r_{a c_{s t 0}}^{3}} \frac{\partial B_{1}}{\partial r}+A_{0}\left(\frac{k}{r_{a c_{s t 0}}^{3}} \frac{\mathrm{d} r_{a c_{s t 0}}}{\mathrm{~d} x}+u_{s t 0} \frac{\mathrm{d}\left(u_{s t 0} k\right)}{\mathrm{d} x}\right)+A_{0}\left(\omega+u_{s t 0} k\right)\left(\frac{u_{s t 0}}{r_{a c_{s t 0}}} \frac{\mathrm{d} r_{a c_{s t 0}}}{\mathrm{~d} x}+\frac{\mathrm{d} u_{s t 0}}{\mathrm{~d} x}\right) \\
+\left(2 \frac{\mathrm{d} r_{a c_{s t 0}}}{\mathrm{~d} x} \frac{\partial A_{0}}{\partial r}+2 \frac{\partial A_{0}}{\partial x}+\frac{3 A_{0}}{r_{a c_{s t 0}}} \frac{\mathrm{d} r_{a c_{s t 0}}}{\mathrm{~d} x}\right) u_{s t 0}\left(\omega+u_{s t 0} k\right)=0 \quad \text { en } \quad r=r_{a c_{s t 0}} \quad \text { (C.57) } \\
F \frac{\partial B_{1}}{\partial r}=-A_{0} k \frac{\mathrm{d} R_{w}}{\mathrm{~d} x} \quad \text { en } r=R_{w}
\end{gathered}
$$

Introduciendo el valor de $A_{0}$ dado por (C.45), las ecuaciones (C.56) - (C.58) quedan de la siguiente forma:

$$
\begin{gathered}
\frac{1}{r F} \frac{\partial}{\partial r}\left(r F^{2} \frac{\partial B_{1}}{\partial r}\right)=2 k\left(F \frac{\mathrm{d} a}{\mathrm{~d} x}+a \frac{\partial F}{\partial x}\right)+a F \frac{\mathrm{d} k}{\mathrm{~d} x} \\
\frac{\partial B_{1}}{\partial r}=-\frac{r_{a c_{s t 0}}^{3}}{F}\left(2 a \frac{\mathrm{d} r_{a c_{s t 0} 0}}{\mathrm{~d} x} \frac{\partial F}{\partial r}+2 a \frac{\partial F}{\partial x}+2 F \frac{\mathrm{d} a}{\mathrm{~d} x}+\frac{3 a F}{r_{a c_{s t 0}}} \frac{\mathrm{d} r_{a c_{s t 0}}}{\mathrm{~d} x}\right) u_{s t 0}\left(\omega+u_{s t 0} k\right) \\
-a r_{a c_{s t 0}}^{3}\left(\frac{k}{r_{a c_{s t 0}}^{3}} \frac{\mathrm{d} r_{a c_{s t 0}}}{\mathrm{~d} x}+u_{s t 0} \frac{\mathrm{d}\left(u_{s t 0} k\right)}{\mathrm{d} x}\right) \\
-a r_{a c_{s t 0}}^{3}\left(\omega+u_{s t 0} k\right)\left(\frac{u_{s t 0}}{r_{a c_{s t 0}}} \frac{\mathrm{d} r_{a c_{s t 0}}}{\mathrm{~d} x}+\frac{\mathrm{d} u_{s t 0}}{\mathrm{~d} x}\right) \quad \text { en } \quad r=r_{a c_{s t 0}}
\end{gathered}
$$




$$
\frac{\partial B_{1}}{\partial r}=-a k \frac{\mathrm{d} R_{w}}{\mathrm{~d} x} \quad \text { en } \quad r=R_{w}
$$

Multiplicando (C.59) por $r F$, integrando entre $r=r_{a c}$ y $r=R_{w}$, y teniendo en cuenta los valores que toma $\partial B_{1} / \partial r$ en los límites de integración, dados por (C.60) y (C.61), se obtiene:

$$
\begin{gathered}
-a k R_{w} \frac{\mathrm{d} R_{w}}{\mathrm{~d} x} F\left(R_{w}\right)^{2}+a r_{a c_{s t 0}}^{4} F\left(r_{a c_{s t 0}}\right)^{2}\left(\frac{k}{r_{a c_{s t 0}}^{3}} \frac{\mathrm{d} r_{a c_{s t 0}}}{\mathrm{~d} x}+u_{s t 0} \frac{\mathrm{d}\left(u_{s t 0} k\right)}{\mathrm{d} x}\right) \\
+r_{a c_{s t 0}}^{4} F\left(r_{a c_{s t 0}}\right)\left(2 a \frac{\mathrm{d} r_{a c_{s t 0} 0} \frac{\partial F}{\partial r}}{\mathrm{~d} x}+2 a \frac{\partial F}{\partial x}+2 F \frac{\mathrm{d} a}{\mathrm{~d} x}+\frac{3 a F}{r_{a c_{s t 0}}} \frac{\mathrm{d} r_{a c_{s t 0}}}{\mathrm{~d} x}\right)_{r=r_{a c_{s t 0}}} u_{s t 0}\left(\omega+u_{s t 0} k\right) \\
+a r_{a c_{s t 0}}^{4} F\left(r_{a c_{s t 0}}\right)^{2}\left(\omega+u_{s t 0} k\right)\left(\frac{u_{s t 0}}{r_{a c_{s t 0}}} \frac{\mathrm{d} r_{a c_{s t 0}}}{\mathrm{~d} x}+\frac{\mathrm{d} u_{s t 0}}{\mathrm{~d} x}\right) \\
=2 k \int_{r_{a c_{s t 0}}}^{R_{w}}\left(F \frac{\mathrm{d} a}{\mathrm{~d} x}+a \frac{\partial F}{\partial x}\right) F r \mathrm{~d} r+a \frac{\mathrm{d} k}{\mathrm{~d} x} \int_{r_{a c_{s t 0}}}^{R_{w}} F^{2} r \mathrm{~d} r
\end{gathered}
$$

Operando se llega finalmente a la siguiente expresión:

$$
\frac{\partial}{\partial x}\left[a^{2}\left(k \int_{r_{a c_{s t} 0}}^{R_{w}} F^{2} r \mathrm{~d} r-F^{2}\left(x, r_{a c_{s t 0}}\right) r_{a c_{s t 0}}^{4} u_{s t 0}\left(\omega+u_{s t 0} k\right)\right)\right]=0
$$

Esta expresión es equivalente a:

$$
\frac{\partial}{\partial x}\left(\frac{\left(u_{s t 0}+c_{g}\right) E}{\omega+u_{s t 0} k}\right)=0
$$

donde $c_{g}$ es la velocidad de grupo y $\left(u_{s t 0}+c_{g}\right) E$ es el flujo de energía de las ondas, que se define, de acuerdo con Lighthill (1978), de la siguiente manera:

$$
\left(u_{s t 0}+c_{g}\right) E=\int_{r_{a c_{s t}+\eta}}^{R_{w}} \overline{p_{p} \frac{\partial \varphi}{\partial x}} 2 \pi r \mathrm{~d} r
$$

donde la línea sobre las variables indica valor promedio, y $p_{p}$ es la perturbación en la presión, que viene dada por:

$$
p_{p}=-\frac{\partial \varphi_{p}}{\partial t}-\frac{\partial \varphi_{s t}}{\partial r} \frac{\partial \varphi_{p}}{\partial r}-\frac{1}{\beta^{2}} \frac{\partial \varphi_{s t}}{\partial x} \frac{\partial \varphi_{p}}{\partial x}
$$

Efectivamente, descomponiendo la integral del segundo miembro de (C.64):

$$
\begin{aligned}
& \int_{r_{a c_{s t}+\eta}}^{R_{w}} \overline{p_{p} \frac{\partial \varphi}{\partial x}} 2 \pi r \mathrm{~d} r=\int_{r_{a c_{s t}}}^{R_{w}} \overline{p_{p} \frac{\partial \varphi_{p}}{\partial x}} 2 \pi r \mathrm{~d} r+\int_{r_{a c_{s t}+\eta}}^{r_{a c_{s t} t}} \overline{p_{p} \frac{\partial \varphi_{p}}{\partial x}} 2 \pi r \mathrm{~d} r \\
& +\int_{r_{a c_{s t}}}^{R_{w}} \overline{p_{p} \frac{\partial \varphi_{s t}}{\partial x}} 2 \pi r \mathrm{~d} r+\int_{r_{a c_{s t}}+\eta}^{r_{a c_{s t} t}} \overline{p_{p} \frac{\partial \varphi_{s t}}{\partial x}} 2 \pi r \mathrm{~d} r
\end{aligned}
$$


Eliminando en el segundo miembro la segunda integral, por ser de mayor orden en potencias de la amplitud de las perturbaciones, y la tercera, por dar lugar a un término oscilatorio:

$$
\int_{r_{a c_{s t}+\eta}}^{R_{w}} \overline{p_{p} \frac{\partial \varphi}{\partial x}} 2 \pi r \mathrm{~d} r=\int_{r_{a c_{s t}}}^{R_{w}} \overline{p_{p} \frac{\partial \varphi_{p}}{\partial x}} 2 \pi r \mathrm{~d} r+\int_{r_{a c_{s t}+\eta}}^{r_{a c_{s t} t}} \overline{p_{p} \frac{\partial \varphi_{s t}}{\partial x}} 2 \pi r \mathrm{~d} r
$$

La última integral del segundo miembro de (C.66) puede aproximarse de la siguiente manera:

$$
\int_{r_{a c_{s t}}+\eta}^{r_{a c_{s t} t}} \overline{p_{p} \frac{\partial \varphi_{s t}}{\partial x}} 2 \pi r \mathrm{~d} r \simeq-2 \pi r_{a c_{s t}} \overline{\left(p_{p} \frac{\partial \varphi_{s t}}{\partial x}\right)_{r=r_{a c_{s t}}}}
$$

Introduciendo (C.65) - (C.67) en (C.64):

$$
\begin{aligned}
& \left(u_{s t 0}+c_{g}\right) E=-2 \pi \int_{r_{a c_{s t}}}^{R_{w}}\left[\overline{\frac{\partial \varphi_{p}}{\partial t} \frac{\partial \varphi_{p}}{\partial x}}+\frac{\partial \varphi_{s t}}{\partial r} \frac{\overline{\partial \varphi_{p}}}{\partial r} \frac{\partial \varphi_{p}}{\partial x}+\frac{1}{\beta^{2}} \frac{\partial \varphi_{s t}}{\partial x} \overline{\left(\frac{\partial \varphi_{p}}{\partial x}\right)^{2}}\right] r \mathrm{~d} r \\
& +2 \pi r_{a c_{s t}}\left(\frac{\partial \varphi_{s t}}{\partial x}\right)_{r=r_{a c_{s t}}}\left[\overline{\eta \frac{\partial \varphi_{p}}{\partial t}}+\frac{\partial \varphi_{s t}}{\partial r} \overline{\eta \frac{\partial \varphi_{p}}{\partial r}}+\frac{1}{\beta^{2}} \frac{\partial \varphi_{s t}}{\partial x} \overline{\eta \frac{\partial \varphi_{p}}{\partial x}}\right]_{r=r_{a c_{s t}}}
\end{aligned}
$$

Teniendo en cuenta (C.26), (C.31), (C.36), (C.45) y (C.47), los valores de la perturbación del potencial de velocidades, $\varphi_{p}$, y de la deformación, $\eta$, son:

$$
\begin{gathered}
\varphi_{p}=a F e^{i \beta s+i \omega t}+a^{*} F e^{-i \beta s-i \omega t} \\
\eta=i r_{a c_{s t 0}}^{3} a F\left(r_{a c_{s t 0}}\right)\left(\omega+u_{s t 0} k\right) e^{i \beta s+i \omega t}-i r_{a c_{s t 0}}^{3} a^{*} F\left(r_{a c_{s t 0}}\right)\left(\omega+u_{s t 0} k\right) e^{-i \beta s-i \omega t}
\end{gathered}
$$

donde $a^{*}$ es el complejo conjugado de $a$, y $F$ viene dada por (C.46). Así pues, se obtiene:

$$
\begin{aligned}
& \frac{\partial \varphi_{p}}{\partial t} \frac{\partial \varphi_{p}}{\partial x}=i \omega a \frac{\mathrm{d} a}{\mathrm{~d} x} F^{2} e^{2 i \beta s+2 i \omega t}+i \omega a^{2} F \frac{\partial F}{\partial x} e^{2 i \beta s+2 i \omega t}-\beta \omega k a^{2} F^{2} e^{2 i \beta s+2 i \omega t} \\
& +\beta 2 \omega k|a|^{2} F^{2}+i \omega a \frac{\mathrm{d} a^{*}}{\mathrm{~d} x} F^{2}-i \omega a^{*} \frac{\mathrm{d} a}{\mathrm{~d} x} F^{2} \\
& -i \omega a^{*} \frac{\mathrm{d} a^{*}}{\mathrm{~d} x} F^{2} e^{-2 i \beta s-2 i \omega t}-i \omega a^{* 2} F \frac{\partial F}{\partial x} e^{-2 i \beta s-2 i \omega t}-\beta \omega k a^{* 2} F^{2} e^{-2 i \beta s-2 i \omega t} \\
& \frac{\partial \varphi_{p}}{\partial r} \frac{\partial \varphi_{p}}{\partial x}=a \frac{\mathrm{d} a}{\mathrm{~d} x} F \frac{\partial F}{\partial r} e^{2 i \beta s+2 i \omega t}+a^{2} \frac{\partial F}{\partial x} \frac{\partial F}{\partial r} e^{2 i \beta s+2 i \omega t}+\beta i k a^{2} F \frac{\partial F}{\partial r} e^{2 i \beta s+2 i \omega t} \\
& +2|a|^{2} \frac{\partial F}{\partial x} \frac{\partial F}{\partial r}+a \frac{\mathrm{d} a^{*}}{\mathrm{~d} x} F \frac{\partial F}{\partial r}+a^{*} \frac{\mathrm{d} a}{\mathrm{~d} x} F \frac{\partial F}{\partial r} \\
& +a^{*} \frac{\mathrm{d} a^{*}}{\mathrm{~d} x} F \frac{\partial F}{\partial r} e^{-2 i \beta s-2 i \omega t}+a^{* 2} \frac{\partial F}{\partial x} \frac{\partial F}{\partial r} e^{-2 i \beta s-2 i \omega t}-\beta i k a^{* 2} F \frac{\partial F}{\partial r} e^{-2 i \beta s-2 i \omega t}
\end{aligned}
$$


C. Propagación de ondas en la superficie del núcleo de aire en una cámara de sección

$$
\begin{aligned}
& \left(\frac{\partial \varphi_{p}}{\partial x}\right)^{2}=\left(\frac{\mathrm{d} a}{\mathrm{~d} x}\right)^{2} F^{2} e^{2 i \beta s+2 i \omega t}+2 a \frac{\mathrm{d} a}{\mathrm{~d} x} F \frac{\partial F}{\partial x} e^{2 i \beta s+2 i \omega t}+2 \beta i k a \frac{\mathrm{d} a}{\mathrm{~d} x} F^{2} e^{2 i \beta s+2 i \omega t} \\
& +2 \frac{\mathrm{d} a}{\mathrm{~d} x} \frac{\mathrm{d} a^{*}}{\mathrm{~d} x} F^{2}+2 a^{*} \frac{\mathrm{d} a}{\mathrm{~d} x} F \frac{\partial F}{\partial x}-2 \beta i k a^{*} \frac{\mathrm{d} a}{\mathrm{~d} x} F^{2} \\
& +a^{2}\left(\frac{\partial F}{\partial x}\right)^{2} e^{2 i \beta s+2 i \omega t}+2 \beta i k a^{2} F \frac{\partial F}{\partial x} e^{2 i \beta s+2 i \omega t}+2 a \frac{\mathrm{d} a^{*}}{\mathrm{~d} x} F \frac{\partial F}{\partial x} \\
& +2|a|^{2}\left(\frac{\partial F}{\partial x}\right)^{2}-\beta^{2} k^{2} a^{2} F^{2} e^{2 i \beta s+2 i \omega t}+2 \beta i k a \frac{\mathrm{d} a^{*}}{\mathrm{~d} x} F^{2}+2 \beta^{2} k^{2}|a|^{2} F^{2} \\
& +\left(\frac{\mathrm{d} a^{*}}{\mathrm{~d} x}\right)^{2} F^{2} e^{-2 i \beta s-2 i \omega t}+2 a^{*} \frac{\mathrm{d} a^{*}}{\mathrm{~d} x} F \frac{\partial F}{\partial x} e^{-2 i \beta s-2 i \omega t}-2 \beta i k a^{*} \frac{\mathrm{d} a^{*}}{\mathrm{~d} x} F^{2} e^{-2 i \beta s-2 i \omega t} \\
& +a^{* 2}\left(\frac{\partial F}{\partial x}\right)^{2} e^{-2 i \beta s-2 i \omega t}-2 \beta i k a^{* 2} F \frac{\partial F}{\partial x} e^{-2 i \beta s-2 i \omega t}-\beta^{2} k^{2} a^{* 2} F^{2} e^{-2 i \beta s-2 i \omega t} \\
& \left(\eta \frac{\partial \varphi_{p}}{\partial t}\right)_{r=r_{a c_{s t}}}=-\omega r_{a c_{s t 0}}^{3} a^{2} F^{2}\left(r_{a c_{s t 0}}\right)\left(\omega+u_{s t 0} k\right) e^{2 i \beta s+2 i \omega t} \\
& +2 \omega r_{a c_{s t 0}}^{3}|a|^{2} F^{2}\left(r_{a c_{s t 0}}\right)\left(\omega+u_{s t 0} k\right)-\omega r_{a c_{s t 0}}^{3} a^{* 2} F^{2}\left(r_{a c_{s t 0}}\right)\left(\omega+u_{s t 0} k\right) e^{-2 i \beta s-2 i \omega t} \\
& \left(\eta \frac{\partial \varphi_{p}}{\partial r}\right)_{r=r_{a c_{s t}}}=i r_{a c_{s t 0}}^{3} a^{2} F\left(r_{a c_{s t 0}}\right)\left(\frac{\partial F}{\partial r}\right)_{r=r_{a c_{s t 0}}}\left(\omega+u_{s t 0} k\right) e^{2 i \beta s+2 i \omega t} \\
& -i r_{a c_{s t 0}}^{3} a^{* 2} F\left(r_{a c_{s t 0}}\right)\left(\frac{\partial F}{\partial r}\right)_{r=r_{a c_{s t 0}}}\left(\omega+u_{s t 0} k\right) e^{-2 i \beta s-2 i \omega t} \\
& \left(\eta \frac{\partial \varphi_{p}}{\partial x}\right)_{r=r_{a c} s t}=i r_{c_{s t 0}}^{3} a \frac{\mathrm{d} a}{\mathrm{~d} x} F^{2}\left(r_{a c_{s t 0}}\right)\left(\omega+u_{s t 0} k\right) e^{2 i \beta s+2 i \omega t} \\
& +i r_{a c_{s t 0}}^{3} a^{2} F\left(r_{a c_{s t 0}}\right)\left(\frac{\partial F}{\partial x}\right)_{r=r_{a c_{s t 0}}}\left(\omega+u_{s t 0} k\right) e^{2 i \beta s+2 i \omega t}-\beta k r_{a c_{s t 0}}^{3} a^{2} F^{2}\left(r_{a c_{s t 0}}\right)\left(\omega+u_{s t 0} k\right) e^{2 i \beta s+2 i \omega t} \\
& +i r_{a c_{s t 0}}^{3} a \frac{\mathrm{d} a^{*}}{\mathrm{~d} x} F^{2}\left(r_{a c_{s t 0}}\right)\left(\omega+u_{s t 0} k\right)+2 \beta k r_{a c_{s t 0}}^{3}|a|^{2} F^{2}\left(r_{a c_{s t 0}}\right)\left(\omega+u_{s t 0} k\right) \\
& -i r_{a c_{s t 0}}^{3} a^{*} \frac{\mathrm{d} a}{\mathrm{~d} x} F^{2}\left(r_{a c_{s t 0}}\right)\left(\omega+u_{s t 0} k\right)-i r_{a c_{s t 0}}^{3} a^{*} \frac{\mathrm{d} a^{*}}{\mathrm{~d} x} F^{2}\left(r_{a c_{s t 0}}\right)\left(\omega+u_{s t 0} k\right) e^{-2 i \beta s-2 i \omega t} \\
& -i r_{a c_{s t 0}}^{3} a^{* 2} F\left(r_{a c_{s t 0}}\right)\left(\frac{\partial F}{\partial x}\right)_{r=r_{a c_{s t 0}}}\left(\omega+u_{s t 0} k\right) e^{-2 i \beta s-2 i \omega t}-\beta k r_{a c_{s t 0}}^{3} a^{* 2} F^{2}\left(r_{a c_{s t 0}}\right)\left(\omega+u_{s t 0} k\right) e^{-2 i \beta s-2 i \omega t}
\end{aligned}
$$

Para el promedio, se eliminan los términos que oscilan:

$$
\begin{aligned}
& \overline{\frac{\partial \varphi_{p}}{\partial t} \frac{\partial \varphi_{p}}{\partial x}}=\beta 2 \omega k|a|^{2} F^{2}+i \omega a \frac{\mathrm{d} a^{*}}{\mathrm{~d} x} F^{2}-i \omega a^{*} \frac{\mathrm{d} a}{\mathrm{~d} x} F^{2} \\
& \overline{\frac{\partial \varphi_{p}}{\partial r} \frac{\partial \varphi_{p}}{\partial x}}=2|a|^{2} \frac{\partial F}{\partial x} \frac{\partial F}{\partial r}+a \frac{\mathrm{d} a^{*}}{\mathrm{~d} x} F \frac{\partial F}{\partial r}+a^{*} \frac{\mathrm{d} a}{\mathrm{~d} x} F \frac{\partial F}{\partial r}
\end{aligned}
$$




$$
\begin{aligned}
& \overline{\left(\frac{\partial \varphi_{p}}{\partial x}\right)^{2}}=2 \frac{\mathrm{d} a}{\mathrm{~d} x} \frac{\mathrm{d} a^{*}}{\mathrm{~d} x} F^{2}+2 a^{*} \frac{\mathrm{d} a}{\mathrm{~d} x} F \frac{\partial F}{\partial x}-2 \beta i k a^{*} \frac{\mathrm{d} a}{\mathrm{~d} x} F^{2} \\
& +2 a \frac{\mathrm{d} a^{*}}{\mathrm{~d} x} F \frac{\partial F}{\partial x}+2|a|^{2}\left(\frac{\partial F}{\partial x}\right)^{2}+2 \beta i k a \frac{\mathrm{d} a^{*}}{\mathrm{~d} x} F^{2}+2 \beta^{2} k^{2}|a|^{2} F^{2} \\
& \overline{\left(\eta \frac{\partial \varphi_{p}}{\partial t}\right)_{r=r_{a c_{s t}}}}=2 \omega r_{a c_{s t 0}}^{3}|a|^{2} F^{2}\left(r_{a c_{s t 0}}\right)\left(\omega+u_{s t 0} k\right) \\
& \overline{\left(\eta \frac{\partial \varphi_{p}}{\partial r}\right)_{r=r_{a c_{s t}}}}=0 \\
& \overline{\left(\eta \frac{\partial \varphi_{p}}{\partial x}\right)_{r=r_{a c_{s t}}}}=i r_{a c_{s t 0}}^{3} a \frac{\mathrm{d} a^{*}}{\mathrm{~d} x} F^{2}\left(r_{a c_{s t 0}}\right)\left(\omega+u_{s t 0} k\right) \\
& +2 \beta k r_{a c_{s t 0}}^{3}|a|^{2} F^{2}\left(r_{a c_{s t 0}}\right)\left(\omega+u_{s t 0} k\right)-i r_{a c_{s t 0}}^{3} a^{*} \frac{\mathrm{d} a}{\mathrm{~d} x} F^{2}\left(r_{a c_{s t 0}}\right)\left(\omega+u_{s t 0} k\right)
\end{aligned}
$$

Por otro lado, las derivadas del potencial de velocidades en régimen estacionario, $\varphi_{s t}$, son:

$$
\begin{gathered}
\frac{\partial \varphi_{s t}}{\partial x}=\beta u_{s t 0} \\
\frac{\partial \varphi_{s t}}{\partial r}=\frac{1}{\beta} u_{s t 0} \frac{\mathrm{d} r_{a c_{s t 0}}}{\mathrm{~d} x}
\end{gathered}
$$

Introduciendo (C.71) - (C.78) en (C.68), y tomando los términos de mayor orden de $\beta$ :

$$
\left(u_{s t 0}+c_{g}\right) E=-\beta 4 \pi\left(\omega+u_{s t 0} k\right)|a|^{2}\left(k \int_{r_{a c_{s t}}}^{R_{w}} F^{2} r \mathrm{~d} r-F^{2}\left(r_{a c_{s t 0}}\right) r_{a c_{s t}}^{4} u_{s t 0}\left(\omega+u_{s t 0} k\right)\right)
$$

Por lo que:

$$
\frac{\left(u_{s t 0}+c_{g}\right) E}{\omega+u_{s t 0} k}=-\beta 4 \pi|a|^{2}\left(k \int_{r_{a c_{s t}}}^{R_{w}} F^{2} r \mathrm{~d} r-F^{2}\left(r_{a c_{s t 0}}\right) r_{a c_{s t}}^{4} u_{s t 0}\left(\omega+u_{s t 0} k\right)\right)
$$

Así pues, las expresiones (C.62) y (C.63) son equivalentes. 
C. Propagación de ondas en la superficie del núcleo de aire en una cámara de sección 148 lentamente variable 


\section{Apéndice D}

\section{Lámina líquida estacionaria}

En este Apéndice se incluye el desarrollo del análisis de la forma estacionaria de la lámina generada a la salida del atomizador, presentado en la Sección 4.1. El problema a resolver es un problema axilsimétrico gobernado por las siguientes ecuaciones, en coordenadas esféricas:

$$
\begin{gathered}
\frac{1}{r^{2}} \frac{\partial}{\partial r}\left(r^{2} \frac{\partial \varphi_{s t}}{\partial r}\right)+\frac{1}{r^{2} \sin \theta} \frac{\partial}{\partial \theta}\left(\sin \theta \frac{\partial \varphi_{s t}}{\partial \theta}\right)=0 \\
\frac{1}{2}\left[\left(\frac{\partial \varphi_{s t}}{\partial r}\right)^{2}+\left(\frac{1}{r} \frac{\partial \varphi_{s t}}{\partial \theta}\right)^{2}+\left(\frac{\Gamma}{2 \pi r \sin \theta}\right)^{2}\right]+\frac{p_{s t}}{\rho}=\frac{1}{2} U_{\infty}^{2}+\frac{p_{a}}{\rho} \\
r \frac{\partial\left(\theta_{c}+\Delta \theta_{s_{s t \pm}}\right.}{\partial r} \frac{\partial \varphi_{s t}}{\partial r}=\frac{1}{r} \frac{\partial \varphi_{s t}}{\partial \theta} \quad \text { en } \quad \theta=\theta_{c}+\Delta \theta_{s_{s t \pm}} \\
p_{s t}-p_{a}=\sigma \nabla \cdot \mathbf{n}_{ \pm} \text {en } \quad \theta=\theta_{c}+\Delta \theta_{s_{s t \pm}}
\end{gathered}
$$

donde $\varphi_{s t} \mathrm{y} p_{s t}$ son el potencial de velocidades y la presión en el líquido, respectivamente, en régimen estacionario, $p_{a}$ es la presión del gas del ambiente, $\rho$ es la densidad del líquido, $\sigma$ es la tensión superficial, $\theta_{c}(r)$ es el ángulo de la línea media de la lámina con el eje de simetría, $\Delta \theta_{s_{s t \pm}}$ es la variación del ángulo respecto al de la línea media al que se encuentran las superficies exterior e interior de la lámina líquida y $\mathbf{n}_{ \pm}$es el vector normal a cada una de esas superficies, orientado hacia el gas. La divergencia de dicho vector es:

$$
\begin{aligned}
\nabla \cdot \mathbf{n}= \pm \frac{1}{r^{2}} \frac{\partial}{\partial r}\left[r^{3} \frac{\partial\left(\theta_{c}+\Delta \theta_{s_{s t \pm}}\right)}{\partial r}\right. & \left.\left(1+r^{2}\left(\frac{\partial\left(\theta_{c}+\Delta \theta_{s_{s t \pm}}\right)}{\partial r}\right)^{2}\right)^{-1 / 2}\right] \\
& -\frac{\cot \left(\theta_{c}+\Delta \theta_{s_{s t \pm}}\right)}{r}\left[1+r^{2}\left(\frac{\partial\left(\theta_{c}+\Delta \theta_{s_{s t \pm}}\right)}{\partial r}\right)^{2}\right]^{-1 / 2}
\end{aligned}
$$

Se define el número de Weber $W=\rho U_{\infty}^{2} R_{0} / \sigma$. En lo que sigue $r$ se adimensionaliza con $W R_{0}$, el potencial de velocidades con $W U_{\infty} R_{0}$ y $p-p_{a}$ con $\rho U_{\infty}^{2}$. Escribiendo $\theta=$ 
$\theta_{c}+W^{-2} \tilde{\theta}$, las derivadas parciales del potencial de velocidades quedan:

$$
\frac{\partial \varphi_{s t}}{\partial r}=\frac{\partial \varphi_{s t}}{\partial r}-W^{2} \frac{\mathrm{d} \theta_{c}}{\mathrm{~d} r} \frac{\partial \varphi_{s t}}{\partial \tilde{\theta}} \quad ; \quad \frac{\partial \varphi_{s t}}{\partial \theta}=W^{2} \frac{\partial \varphi_{s t}}{\partial \tilde{\theta}}
$$

Las ecuaciones (D.1) - (D.4) toman la forma:

$$
\begin{aligned}
& W^{4}\left[\left(\frac{\mathrm{d} \theta_{c}}{\mathrm{~d} r}\right)^{2}+\frac{1}{r^{2}}\right] \frac{\partial^{2} \varphi_{s t}}{\partial \tilde{\theta}^{2}}-W^{2}\left[\frac{1}{r^{2}} \frac{\mathrm{d}}{\mathrm{d} r}\left(r^{2} \frac{\mathrm{d} \theta_{c}}{\mathrm{~d} r}\right) \frac{\partial \varphi_{s t}}{\partial \tilde{\theta}}+2 \frac{\mathrm{d} \theta_{c}}{\mathrm{~d} r} \frac{\partial^{2} \varphi_{s t}}{\partial r \partial \tilde{\theta}}-\frac{\cot \theta_{c}}{r^{2}} \frac{\partial \varphi_{s t}}{\partial \tilde{\theta}}\right] \\
& +\frac{1}{r^{2}} \frac{\partial}{\partial r}\left(r^{2} \frac{\partial \varphi_{s t}}{\partial r}\right)-\frac{\tilde{\theta}}{r^{2} \sin ^{2} \theta_{c}} \frac{\partial \varphi_{s t}}{\partial \tilde{\theta}}+O\left(W^{-2}\right)=0 \\
& W^{4} \frac{1}{2}\left[\left(\frac{\mathrm{d} \theta_{c}}{\mathrm{~d} r}\right)^{2}+\frac{1}{r^{2}}\right]\left(\frac{\partial \varphi_{s t}}{\partial \tilde{\theta}}\right)^{2}-W^{2} \frac{\mathrm{d} \theta_{c}}{\mathrm{~d} r} \frac{\partial \varphi_{s t}}{\partial r} \frac{\partial \varphi_{s t}}{\partial \tilde{\theta}}+\left[\frac{1}{2}\left(\frac{\partial \varphi_{s t}}{\partial r}\right)^{2}+p_{s t}-\frac{1}{2}\right] \\
& +W^{-2} \frac{\gamma^{2}}{2 r^{2} \sin ^{2} \theta_{c}}-W^{-4} \frac{\gamma^{2} \tilde{\theta} \cos \theta_{c}}{r^{2} \sin ^{3} \theta_{c}}+O\left(W^{-6}\right)=0 \\
& W^{2}\left[\left(\frac{\mathrm{d} \theta_{c}}{\mathrm{~d} r}\right)^{2}+\frac{1}{r^{2}}\right] \frac{\partial \varphi_{s t}}{\partial \tilde{\theta}}+\frac{\mathrm{d} \theta_{c}}{\mathrm{~d} r}\left(\frac{\partial \tilde{\theta}_{s_{s t \pm}}}{\partial r} \frac{\partial \varphi_{s t}}{\partial \tilde{\theta}}-\frac{\partial \varphi_{s t}}{\partial r}\right)-W^{-2} \frac{\partial \tilde{\theta}_{s_{s t \pm}}}{\partial r} \frac{\partial \varphi_{s t}}{\partial r}=0 \\
& \text { en } \quad \theta=\theta_{c}+W^{-2} \tilde{\theta}_{s s t \pm} \\
& p_{s t} \pm W^{-2}\left[\frac{1}{r^{2}} \frac{\partial}{\partial r}\left(\frac{r^{3} \frac{\mathrm{d} \theta_{c}}{\mathrm{~d} r}}{\left(1+r^{2}\left(\frac{\mathrm{d} \theta_{c}}{\mathrm{~d} r}+W^{-2} \frac{\partial \tilde{\theta}_{s t \pm}}{\partial r}\right)^{2}\right)^{1 / 2}}\right)-\frac{\frac{\cot \theta_{c}}{r}}{\left(1+r^{2}\left(\frac{\mathrm{d} \theta_{c}}{\mathrm{~d} r}+W^{-2} \frac{\partial \tilde{\theta}_{s t \pm}}{\partial r}\right)^{2}\right)^{1 / 2}}\right] \\
& \pm W^{-4}\left[\frac{1}{r^{2}} \frac{\partial}{\partial r}\left(\frac{r^{3} \frac{\partial \tilde{\theta}_{s t \pm}}{\partial r}}{\left(1+r^{2}\left(\frac{\mathrm{d} \theta_{c}}{\mathrm{~d} r}+W^{-2} \frac{\partial \tilde{\theta}_{s s t \pm}}{\partial r}\right)^{2}\right)^{1 / 2}}\right)+\frac{\frac{\tilde{\theta}_{s_{s t \pm}}}{r \sin ^{2} \theta_{c}}}{\left(1+r^{2}\left(\frac{\mathrm{d} \theta_{c}}{\mathrm{~d} r}+W^{-2} \frac{\partial \tilde{\theta}_{s_{s t \pm}}}{\partial r}\right)^{2}\right)^{1 / 2}}\right] \\
& +O\left(W^{-6}\right)=0 \quad \text { en } \quad \theta=\theta_{c}+W^{-2} \tilde{\theta}_{s_{s t \pm}}
\end{aligned}
$$

donde $\gamma=\Gamma /\left(2 \pi R_{0} U_{\infty}\right)=(\pi / 4) /\left(c_{d} / \Delta\right)$, con $c_{d}$ y $\Delta$ el coeficiente de descarga y la constante del atomizador definidos en (2.12) y (2.17), respectivamente, $\tilde{\theta}_{s_{s t \pm}}$ es el valor estacionario de $\tilde{\theta}$ en las superficies exterior e interior de la lámina líquida, y en donde se ha tenido en cuenta que:

$$
\cot \theta=\cot \theta_{c}-W^{-2} \frac{\tilde{\theta}}{\sin ^{2} \theta_{c}}+O\left(W^{-4}\right)
$$




$$
\frac{1}{\sin ^{2} \theta}=\frac{1}{\sin ^{2} \theta_{c}}-W^{-2} \frac{2 \tilde{\theta} \cos \theta_{c}}{\sin ^{3} \theta_{c}}+O\left(W^{-4}\right)
$$

Consideraremos que el potencial de velocidades y la presión en régimen estacionario admiten los siguientes desarrollos:

$$
\begin{gathered}
\varphi_{s t}=\varphi_{s t 0}+W^{-2} \varphi_{s t 1}+W^{-4} \varphi_{s t 2}+O\left(W^{-6}\right) \\
p_{s t}=p_{s t 0}+W^{-2} p_{s t 1}+W^{-4} p_{s t 2}+O\left(W^{-6}\right)
\end{gathered}
$$

Sustituimos los anteriores desarrollos en (D.6) - (D.9) y agrupamos términos del mismo orden. Tomando los términos de orden $O\left(W^{4}\right)$ de la ecuación (D.6):

$$
\frac{\partial^{2} \varphi_{s t 0}}{\partial \tilde{\theta}^{2}}=0
$$

de donde se obtiene:

$$
\varphi_{s t 0}=A_{0}(r)+B_{0}(r) \tilde{\theta}
$$

Agrupando los términos de orden $O\left(W^{4}\right)$ de la ecuación (D.7):

$$
\frac{\partial \varphi_{s t 0}}{\partial \tilde{\theta}}=0
$$

por lo que:

$$
B_{0}(r)=0
$$

Así pues:

$$
\varphi_{s t 0}=A_{0}(r)
$$

Tomando ahora los términos de orden $O\left(W^{2}\right)$ de la ecuación (D.6), y teniendo en cuenta (D.10):

$$
\frac{\partial^{2} \varphi_{s t 1}}{\partial \tilde{\theta}^{2}}=0
$$

que da lugar a:

$$
\varphi_{s t 1}=A_{1}(r)+B_{1}(r) \tilde{\theta}
$$

Agrupando los términos de orden $O(1)$ de la ecuación (D.7), e introduciendo los resultados obtenidos en (D.10) y (D.11):

$$
\frac{1}{2}\left[\left(\frac{\mathrm{d} \theta_{c}}{\mathrm{~d} r}\right)^{2}+\frac{1}{r^{2}}\right] B_{1}^{2}-\frac{\mathrm{d} \theta_{c}}{\mathrm{~d} r} \frac{\mathrm{d} A_{0}}{\mathrm{~d} r} B_{1}+\frac{1}{2}\left(\frac{\mathrm{d} A_{0}}{\mathrm{~d} r}\right)^{2}+p_{s t 0}-\frac{1}{2}=0
$$

En la expresión (D.12) se observa que $p_{\text {st } 0}$ es independiente de $\tilde{\theta}$. Debido a ello, podemos evaluarla en la superficie de la lámina. Tomando términos de orden $O(1)$ de (D.9):

$$
p_{s t 0}=0
$$


Agrupando los términos de orden $O(1)$ de la ecuación (D.8), y teniendo en cuenta (D.10) y (D.11):

$$
\left[\left(\frac{\mathrm{d} \theta_{c}}{\mathrm{~d} r}\right)^{2}+\frac{1}{r^{2}}\right] B_{1}-\frac{\mathrm{d} \theta_{c}}{\mathrm{~d} r} \frac{\mathrm{d} A_{0}}{\mathrm{~d} r}=0
$$

Las expresiones (D.12) y (D.14) forman un sistema de dos ecuaciones con dos incógnitas, $\mathrm{d} A_{0} / \mathrm{d} r$ y $B_{1}$. Resolviéndolo se obtiene:

$$
\begin{aligned}
& \frac{\mathrm{d} A_{0}}{\mathrm{~d} r}=r\left[\left(\frac{\mathrm{d} \theta_{c}}{\mathrm{~d} r}\right)^{2}+\frac{1}{r^{2}}\right]^{1 / 2} \\
& B_{1}=\frac{\mathrm{d} \theta_{c}}{\mathrm{~d} r} r\left[\left(\frac{\mathrm{d} \theta_{c}}{\mathrm{~d} r}\right)^{2}+\frac{1}{r^{2}}\right]^{-1 / 2}
\end{aligned}
$$

La velocidad del líquido en la dirección de la lámina, $U$, es:

$$
\begin{aligned}
U=\left(\frac{\partial \varphi_{s t}}{\partial r}-W^{2} \frac{\mathrm{d} \theta_{c}}{\mathrm{~d} r} \frac{\partial \varphi_{s t}}{\partial \tilde{\theta}}\right) \cos \phi-W^{2} \frac{1}{r} \frac{\partial \varphi_{s t}}{\partial \tilde{\theta}} \sin \phi= & \\
& \left(\frac{\mathrm{d} A_{0}}{\mathrm{~d} r}-\frac{\mathrm{d} \theta_{c}}{\mathrm{~d} r} B_{1}\right) \cos \phi-\frac{1}{r} B_{1} \sin \phi+O\left(W^{-2}\right)
\end{aligned}
$$

donde (ver Sección 4.1):

$$
\cos \phi=\left[1+r^{2}\left(\frac{\mathrm{d} \theta_{c}}{\mathrm{~d} r}\right)^{2}\right]^{-1 / 2} \quad ; \quad \sin \phi=r \frac{\mathrm{d} \theta_{c}}{\mathrm{~d} r}\left[1+r^{2}\left(\frac{\mathrm{d} \theta_{c}}{\mathrm{~d} r}\right)^{2}\right]^{-1 / 2}
$$

Sustituyendo (D.15), (D.16) y (D.18) en (D.17) se obtiene:

$$
U=1+O\left(W^{-2}\right)
$$

Tomando ahora los términos de orden $O(1)$ de la ecuación (D.6), y teniendo en cuenta (D.10) y (D.11):

$$
\left[\left(\frac{\mathrm{d} \theta_{c}}{\mathrm{~d} r}\right)^{2}+\frac{1}{r^{2}}\right] \frac{\partial^{2} \varphi_{s t 2}}{\partial \tilde{\theta}^{2}}-\frac{1}{r^{2}} \frac{\mathrm{d}}{\mathrm{d} r}\left(r^{2} \frac{\mathrm{d} \theta_{c}}{\mathrm{~d} r}\right) B_{1}-2 \frac{\mathrm{d} \theta_{c}}{\mathrm{~d} r} \frac{\mathrm{d} B_{1}}{\mathrm{~d} r}+\frac{\cot \theta_{c}}{r^{2}} B_{1}+\frac{1}{r^{2}} \frac{\partial}{\partial r}\left(r^{2} \frac{\mathrm{d} A_{0}}{\mathrm{~d} r}\right)=0
$$

Definimos la función $F(r)$ de la siguiente manera:

$$
F(r)=\frac{1}{r^{2}} \frac{\mathrm{d}}{\mathrm{d} r}\left(r^{2} \frac{\mathrm{d} \theta_{c}}{\mathrm{~d} r}\right) B_{1}+2 \frac{\mathrm{d} \theta_{c}}{\mathrm{~d} r} \frac{\mathrm{d} B_{1}}{\mathrm{~d} r}+\frac{\cot \theta_{c}}{r^{2}} B_{1}-\frac{1}{r^{2}} \frac{\partial}{\partial r}\left(r^{2} \frac{\mathrm{d} A_{0}}{\mathrm{~d} r}\right)
$$

por lo que:

$$
\left[\left(\frac{\mathrm{d} \theta_{c}}{\mathrm{~d} r}\right)^{2}+\frac{1}{r^{2}}\right] \frac{\partial^{2} \varphi_{s t 2}}{\partial \tilde{\theta}^{2}}=F(r)
$$


de donde se obtiene la siguiente expresión para $\varphi_{s t 2}$ :

$$
\varphi_{s t 2}=\frac{F(r)}{2}\left[\left(\frac{\mathrm{d} \theta_{c}}{\mathrm{~d} r}\right)^{2}+\frac{1}{r^{2}}\right]^{-1} \tilde{\theta}^{2}+B_{2}(r) \tilde{\theta}+A_{2}(r)
$$

De la ecuación (D.7), agrupando los términos de orden $O\left(W^{-2}\right)$, y con las expresiones (D.10) y (D.11):

$$
\begin{array}{r}
{\left[\left(\frac{\mathrm{d} \theta_{c}}{\mathrm{~d} r}\right)^{2}+\frac{1}{r^{2}}\right] B_{1}\left[F\left(\left(\frac{\mathrm{d} \theta_{c}}{\mathrm{~d} r}\right)^{2}+\frac{1}{r^{2}}\right)^{-1} \tilde{\theta}+B_{2}\right]} \\
-\frac{\mathrm{d} \theta_{c}}{\mathrm{~d} r}\left[\frac{\mathrm{d} A_{0}}{\mathrm{~d} r}\left(F\left(\left(\frac{\mathrm{d} \theta_{c}}{\mathrm{~d} r}\right)^{2}+\frac{1}{r^{2}}\right)^{-1} \tilde{\theta}+B_{2}\right)+\left(\frac{\mathrm{d} A_{1}}{\mathrm{~d} r}+\frac{\mathrm{d} B_{1}}{\mathrm{~d} r} \tilde{\theta}\right) B_{1}\right] \\
+\frac{\mathrm{d} A_{0}}{\mathrm{~d} r}\left(\frac{\mathrm{d} A_{1}}{\mathrm{~d} r}+\frac{\mathrm{d} B_{1}}{\mathrm{~d} r} \tilde{\theta}\right)+p_{s t 1}+\frac{\gamma^{2}}{2 r^{2} \sin ^{2} \theta_{c}}=0
\end{array}
$$

Reordenando los términos:

$$
\begin{aligned}
{\left[\left(\left(\frac{\mathrm{d} \theta_{c}}{\mathrm{~d} r}\right)^{2}+\frac{1}{r^{2}}\right) B_{1}\right.} & \left.-\frac{\mathrm{d} \theta_{c}}{\mathrm{~d} r} \frac{\mathrm{d} A_{0}}{\mathrm{~d} r}\right]\left[F\left(\left(\frac{\mathrm{d} \theta_{c}}{\mathrm{~d} r}\right)^{2}+\frac{1}{r^{2}}\right)^{-1} \tilde{\theta}+B_{2}\right] \\
& -\left(\frac{\mathrm{d} A_{1}}{\mathrm{~d} r}+\frac{\mathrm{d} B_{1}}{\mathrm{~d} r} \tilde{\theta}\right)\left(\frac{\mathrm{d} \theta_{c}}{\mathrm{~d} r} B_{1}-\frac{\mathrm{d} A_{0}}{\mathrm{~d} r}\right)+p_{s t 1}+\frac{\gamma^{2}}{2 r^{2} \sin ^{2} \theta_{c}}=0
\end{aligned}
$$

Teniendo en cuenta (D.14):

$$
p_{s t 1}=\left(\frac{\mathrm{d} A_{1}}{\mathrm{~d} r}+\frac{\mathrm{d} B_{1}}{\mathrm{~d} r} \tilde{\theta}\right)\left(\frac{\mathrm{d} \theta_{c}}{\mathrm{~d} r} B_{1}-\frac{\mathrm{d} A_{0}}{\mathrm{~d} r}\right)-\frac{\gamma^{2}}{2 r^{2} \sin ^{2} \theta_{c}}
$$

De (D.23) se observa que $p_{s t 1}$ es lineal con $\tilde{\theta}$. Como además se ha de cumplir la condición de que $p_{s t 1}\left(\tilde{\theta}_{s_{s t}}\right)=-p_{s t 1}\left(-\tilde{\theta}_{s_{s t}}\right)$, entonces $p_{s t 1}=0$ en $\tilde{\theta}=0$ :

$$
\frac{\mathrm{d} A_{1}}{\mathrm{~d} r}\left(\frac{\mathrm{d} \theta_{c}}{\mathrm{~d} r} B_{1}-\frac{\mathrm{d} A_{0}}{\mathrm{~d} r}\right)=\frac{\gamma^{2}}{2 r^{2} \sin ^{2} \theta_{c}}
$$

Introduciendo en (D.24) los valores de $\mathrm{d} A_{0} / \mathrm{d} r$ y $B_{1}$ dados por (D.15) y (D.16) se obtiene $\mathrm{d} A_{1} / \mathrm{d} r$ :

$$
\frac{\mathrm{d} A_{1}}{\mathrm{~d} r}=\frac{-\gamma^{2}}{2 r \sin ^{2} \theta_{c}}\left[\left(\frac{\mathrm{d} \theta_{c}}{\mathrm{~d} r}\right)^{2}+\frac{1}{r^{2}}\right]^{1 / 2}
$$

y el valor de $p_{s t 1}$ :

$$
p_{s t 1}=-r\left[r \frac{\mathrm{d}^{2} \theta_{c}}{\mathrm{~d} r^{2}}+2 \frac{\mathrm{d} \theta_{c}}{\mathrm{~d} r}+r^{2}\left(\frac{\mathrm{d} \theta_{c}}{\mathrm{~d} r}\right)^{3}\right]\left[1+r^{2}\left(\frac{\mathrm{d} \theta_{c}}{\mathrm{~d} r}\right)^{2}\right]^{-2} \tilde{\theta}
$$


Tomando los términos de orden $O\left(W^{-2}\right)$ de la ecuación (D.8):

$$
\begin{aligned}
{\left[\left(\frac{\mathrm{d} \theta_{c}}{\mathrm{~d} r}\right)^{2}+\frac{1}{r^{2}}\right] } & \left.F\left(\left(\frac{\mathrm{d} \theta_{c}}{\mathrm{~d} r}\right)^{2}+\frac{1}{r^{2}}\right)^{-1} \tilde{\theta}+B_{2}\right] \\
& +\frac{\mathrm{d} \theta_{c}}{\mathrm{~d} r}\left[\frac{\partial \tilde{\theta}_{s_{s t \pm}}}{\partial r} B_{1}-\left(\frac{\mathrm{d} A_{1}}{\mathrm{~d} r}+\frac{\mathrm{d} B_{1}}{\mathrm{~d} r} \tilde{\theta}_{s_{s t \pm}}\right)\right]-\frac{\partial \tilde{\theta}_{s_{s t \pm}}}{\partial r} \frac{\mathrm{d} A_{0}}{\mathrm{~d} r}=0
\end{aligned}
$$

Particularizando (D.27) en las superficies exterior e interior de la lámina líquida:

$$
\begin{array}{r}
F \tilde{\theta}_{s_{s t}}+\left[\left(\frac{\mathrm{d} \theta_{c}}{\mathrm{~d} r}\right)^{2}+\frac{1}{r^{2}}\right] B_{2}+\frac{\mathrm{d} \theta_{c}}{\mathrm{~d} r}\left[\frac{\partial \tilde{\theta}_{s_{s t}}}{\partial r} B_{1}-\left(\frac{\mathrm{d} A_{1}}{\mathrm{~d} r}+\frac{\mathrm{d} B_{1}}{\mathrm{~d} r} \tilde{\theta}_{s_{s t}}\right)\right] \\
-\frac{\partial \tilde{\theta}_{s_{s t}}}{\partial r} \frac{\mathrm{d} A_{0}}{\mathrm{~d} r}=0 \\
-F \tilde{\theta}_{s_{s t}}+\left[\left(\frac{\mathrm{d} \theta_{c}}{\mathrm{~d} r}\right)^{2}+\frac{1}{r^{2}}\right] B_{2}+\frac{\mathrm{d} \theta_{c}}{\mathrm{~d} r}\left[-\frac{\partial \tilde{\theta}_{s_{s t}}}{\partial r} B_{1}-\left(\frac{\mathrm{d} A_{1}}{\mathrm{~d} r}-\frac{\mathrm{d} B_{1}}{\mathrm{~d} r} \tilde{\theta}_{s_{s t}}\right)\right] \\
+\frac{\partial \tilde{\theta}_{s_{s t}}}{\partial r} \frac{\mathrm{d} A_{0}}{\mathrm{~d} r}=0
\end{array}
$$

Restando (D.29) a (D.28):

$$
F \tilde{\theta}_{s_{s t}}+\frac{\mathrm{d} \theta_{c}}{\mathrm{~d} r}\left(\frac{\partial \tilde{\theta}_{s_{s t}}}{\partial r} B_{1}-\frac{\mathrm{d} B_{1}}{\mathrm{~d} r} \tilde{\theta}_{s}\right)-\frac{\partial \tilde{\theta}_{s_{s t}}}{\partial r} \frac{\mathrm{d} A_{0}}{\mathrm{~d} r}=0
$$

Introduciendo (D.15), (D.16) y (D.20) en (D.30) y operando se llega a la siguiente ecuación diferencial para $\tilde{\theta}_{s_{s t}}$ :

$$
\frac{\partial \tilde{\theta}_{s_{s t}}}{\partial r}+\tilde{\theta}_{s_{s t}} \frac{\mathrm{d}}{\mathrm{d} r}\left[r^{2}\left(1+r^{2}\left(\frac{\mathrm{d} \theta_{c}}{\mathrm{~d} r}\right)^{2}\right)^{-1 / 2} \sin \theta_{c}\right]
$$

cuya solución es:

$$
\tilde{\theta}_{s t}=\frac{C^{*}}{r^{2} \sin \theta_{c}}\left[1+r^{2}\left(\frac{\mathrm{d} \theta_{c}}{\mathrm{~d} r}\right)^{2}\right]^{1 / 2}
$$

donde la constante de integración $C^{*}$ se puede determinar por conservación del caudal (escalado con $U_{\infty} R_{0}^{2}$ ), teniendo en cuenta la expresión del coeficiente de descarga definida en (2.12):

$$
\pi c_{d}=U h 2 \pi r \sin \theta_{c}
$$


con $h$ representando el espesor de la lámina, de valor (ver Sección 4.1):

$$
h=2 r \tilde{\theta}_{s t}\left[1+r^{2}\left(\frac{\mathrm{d} \theta_{c}}{\mathrm{~d} r}\right)^{2}\right]^{-1 / 2}
$$

Sustituyendo (D.19), (D.31) y (D.33) en (D.32):

$$
C^{*}=\frac{c_{d}}{4}
$$

y la expresión para $\tilde{\theta}_{s_{s t}}$ resulta:

$$
\tilde{\theta}_{s_{s t}}=\frac{c_{d}}{4 r^{2} \sin \theta_{c}}\left[1+r^{2}\left(\frac{\mathrm{d} \theta_{c}}{\mathrm{~d} r}\right)^{2}\right]^{1 / 2}
$$

Seleccionando los términos de orden $O\left(W^{-2}\right)$ de la ecuación (D.9):

$$
p_{s t 1}+\frac{1}{r^{2}} \frac{\partial}{\partial r}\left[r^{3} \frac{\mathrm{d} \theta_{c}}{\mathrm{~d} r}\left(1+r^{2}\left(\frac{\mathrm{d} \theta_{c}}{\mathrm{~d} r}\right)^{2}\right)^{-1 / 2}\right]-\frac{\cot \theta_{c}}{r}\left[1+r^{2}\left(\frac{\mathrm{d} \theta_{c}}{\mathrm{~d} r}\right)^{2}\right]^{-1 / 2}=0
$$

Sustituyendo (D.26) y (D.34) en (D.35) y operando, se llega finalmente a la siguiente ecuación diferencial para $\theta_{c}$ :

$$
\begin{aligned}
r\left(r \sin \theta_{c}-\frac{c_{d}}{4}\right) \frac{\mathrm{d}^{2} \theta_{c}}{\mathrm{~d} r^{2}}+\left(3 r \sin \theta_{c}-\frac{c_{d}}{2}\right) & \frac{\mathrm{d} \theta_{c}}{\mathrm{~d} r}-r^{2} \cos \theta_{c}\left(\frac{\mathrm{d} \theta_{c}}{\mathrm{~d} r}\right)^{2} \\
& +r^{2}\left(2 r \sin \theta_{c}-\frac{c_{d}}{4}\right)\left(\frac{\mathrm{d} \theta_{c}}{\mathrm{~d} r}\right)^{3}-\cos \theta_{c}=0
\end{aligned}
$$




\section{Apéndice $\mathrm{E}$}

\section{Estabilidad de la lámina líquida}

En este Apéndice se incluye el desarrollo del análisis de la estabilidad de la lámina líquida generada cuando las inestabilidades se producen en la región en que $r \ll W R_{0}$, presentado en la Sección 4.2. En esta región la lámina líquida sin perturbar se puede considerar cónica, luego $\mathrm{d} \theta_{c} / \mathrm{d} r=0$. Introducimos pequeñas perturbaciones a los potenciales de velocidades del líquido y del gas, $\varphi$ y $\varphi_{g}$, a las distribuciones de presión en el líquido y en el gas, $p$ y $p_{g}$, y a la posición de las superficies libres exterior e interior de la lámina líquida, $\tilde{\theta}_{s_{ \pm}}$, de forma que escribimos:

$$
\begin{gathered}
\varphi(r, \tilde{\theta})=\varphi_{s t}(r, \tilde{\theta})+\varphi_{p}(r, \tilde{\theta}, t) \\
p(r, \tilde{\theta})=p_{s t}(r, \tilde{\theta})+p_{p}(r, \tilde{\theta}, t) \\
\tilde{\theta}_{s_{ \pm}}(r)=\tilde{\theta}_{s_{s t \pm}}(r)+\tilde{\theta}_{s_{p \pm}}(r, t) \\
\varphi_{g}(r, \tilde{\theta})=\varphi_{g_{s t}}(r, \tilde{\theta})+\varphi_{g_{p}}(r, \tilde{\theta}, t) \\
p_{g}(r, \tilde{\theta})=p_{g_{s t}}(r, \tilde{\theta})+p_{g_{p}}(r, \tilde{\theta}, t)
\end{gathered}
$$

donde $\left(\varphi_{s t}, p_{s t}, \tilde{\theta}_{s_{s t \pm}}, \varphi_{g_{s t}}, p_{g_{s t}}\right)$ es la solución estacionaria, y $\left(\varphi_{p}, p_{p}, \tilde{\theta}_{s_{p \pm}}, \varphi_{g_{p}}, p_{g_{p}}\right)$ representan las perturbaciones. El problema en la región del líquido está gobernado por las siguientes ecuaciones, en variables adimensionales, con $r$ escalada con $W R_{0}$, el potencial de velocidades con $W U_{\infty} R_{0}, p-p_{a}$ con $\rho U_{\infty}^{2}$, y el tiempo con $W R_{0} / U_{\infty}$ :

$$
\begin{aligned}
W^{4} \frac{1}{r^{2}} \frac{\partial^{2} \varphi}{\partial \tilde{\theta}^{2}}+W^{2} \frac{\cot \theta_{c}}{r^{2}} \frac{\partial \varphi}{\partial \tilde{\theta}}+\frac{1}{r^{2}} \frac{\partial}{\partial r}\left(r^{2} \frac{\partial \varphi}{\partial r}\right) & -\frac{\tilde{\theta}}{r^{2} \sin ^{2} \theta_{c}} \frac{\partial \varphi}{\partial \tilde{\theta}}+O\left(W^{-2}\right)=0 \\
W^{4} \frac{1}{2 r^{2}}\left(\frac{\partial \varphi}{\partial \tilde{\theta}}\right)^{2}+\left[\frac{\partial \varphi}{\partial t}+\frac{1}{2}\left(\frac{\partial \varphi}{\partial r}\right)^{2}+p-\frac{1}{2}\right]+ & W^{-2} \frac{\gamma^{2}}{2 r^{2} \sin ^{2} \theta_{c}} \\
& -W^{-4} \frac{\gamma^{2} \tilde{\theta} \cos \theta_{c}}{r^{2} \sin ^{3} \theta_{c}}+O\left(W^{-6}\right)=0
\end{aligned}
$$




$$
\begin{gathered}
W^{2} \frac{1}{r^{2}} \frac{\partial \varphi}{\partial \tilde{\theta}}-W^{-2}\left(\frac{\partial \tilde{\theta}_{s_{ \pm}}}{\partial t}+\frac{\partial \tilde{\theta}_{s_{ \pm}}}{\partial r} \frac{\partial \varphi}{\partial r}\right)=0 \text { en } \theta=\theta_{c}+W^{-2} \tilde{\theta}_{s_{ \pm}} \\
\left.\left.p-p_{g} \mp W^{-2} \frac{\cot \theta_{c}}{r}\left[1+W^{-4} r^{2}\left(\frac{\partial \tilde{\theta}_{s_{ \pm}}}{\partial r}\right)^{2}\right]^{-1 / 2}\right)+\frac{\frac{\tilde{\theta}_{s_{ \pm}}}{r \sin ^{2} \theta_{c}}}{\left(1+W^{-4} r^{2}\left(\frac{\partial \tilde{\theta}_{s_{ \pm}}}{\partial r}\right)^{2}\right)^{1 / 2}}\right] \\
\pm W^{-4}\left[\frac{1}{r^{2}} \frac{\partial}{\partial r}\left[\frac{r_{s_{ \pm}}}{\left(1+W^{-4} r^{2}\left(\frac{\partial \tilde{\theta}_{s_{ \pm}}}{\partial r}\right)^{2}\right)^{1 / 2}}\right)+O\left(W^{-6}\right)=0 \quad \text { en } \theta=\theta_{c}+W^{-2} \tilde{\theta}_{s_{ \pm}}\right.
\end{gathered}
$$

donde se ha tenido en cuenta que $\mathrm{d} \theta_{c} / \mathrm{d} r=0$. Introduciendo las expresiones dadas en (E.1) en las ecuaciones (E.2) - (E.5) y linealizando:

$$
\begin{aligned}
& W^{4} \frac{1}{r^{2}} \frac{\partial^{2} \varphi_{p}}{\partial \tilde{\theta}^{2}}+W^{2} \frac{\cot \theta_{c}}{r^{2}} \frac{\partial \varphi_{p}}{\partial \tilde{\theta}}+\frac{1}{r^{2}} \frac{\partial}{\partial r}\left(r^{2} \frac{\partial \varphi_{p}}{\partial r}\right)-\frac{\tilde{\theta}}{r^{2} \sin ^{2} \theta_{c}} \frac{\partial \varphi_{p}}{\partial \tilde{\theta}}+O\left(W^{-2}\right)=0 \\
& W^{4} \frac{1}{r^{2}} \frac{\partial \varphi_{s t}}{\partial \tilde{\theta}} \frac{\partial \varphi_{p}}{\partial \tilde{\theta}}+\left(\frac{\partial \varphi_{p}}{\partial t}+\frac{\partial \varphi_{s t}}{\partial r} \frac{\partial \varphi_{p}}{\partial r}+p_{p}\right)+O\left(W^{-4}\right)=0 \\
& W^{2} \frac{1}{r^{2}}\left(\frac{\partial^{2} \varphi_{s t}}{\partial \tilde{\theta}^{2}} \tilde{\theta}_{s_{p \pm}}+\frac{\partial \varphi_{p}}{\partial \tilde{\theta}}\right)-W^{-2}\left[\frac{\partial \tilde{\theta}_{s_{p \pm}}}{\partial t}+\frac{\partial \tilde{\theta}_{s_{p \pm}}}{\partial r} \frac{\partial \varphi_{s t}}{\partial r}+\frac{\partial \tilde{\theta}_{s_{s t \pm}}}{\partial r}\left(\frac{\partial^{2} \varphi_{s t}}{\partial r \partial \tilde{\theta}} \tilde{\theta}_{s_{p \pm}}+\frac{\partial \varphi_{p}}{\partial r}\right)\right] \\
& =0 \quad \text { en } \quad \theta=\theta_{c}+W^{-2} \tilde{\theta}_{s_{ \pm}} \\
& p_{p}+\frac{\partial p_{s t}}{\partial \tilde{\theta}} \tilde{\theta}_{s_{p \pm}}-p_{g_{p}} \pm W^{-4}\left[\frac{1}{r^{2}} \frac{\partial}{\partial r}\left(r^{3} \frac{\partial \tilde{\theta}_{s_{p \pm}}}{\partial r}\right)+\frac{\tilde{\theta}_{s_{p \pm}}}{r \sin ^{2} \theta_{c}}\right] \\
& +O\left(W^{-6}\right)=0 \quad \text { en } \quad \theta=\theta_{c}+W^{-2} \tilde{\theta}_{s_{ \pm}}
\end{aligned}
$$

Consideraremos que las perturbaciones en el potencial de velocidades, en la presión y en la posición de la superficie libre de la lámina líquida admiten los siguientes desarrollos:

$$
\varphi_{p}=W^{-4} \varphi_{p 0}+\ldots \quad ; \quad p_{p}=W^{-4} p_{p 0}+\ldots \quad ; \quad \tilde{\theta}_{s_{p \pm}}=\tilde{\theta}_{s_{p 0 \pm}}+\ldots
$$

Tomando los términos de orden $O(1)$ en la ecuación (E.6):

$$
\frac{\partial^{2} \varphi_{p 0}}{\partial \tilde{\theta}^{2}}=0
$$


de donde se obtiene:

$$
\varphi_{p 0}=A_{p 0}(r, t)+B_{p 0}(r, t) \tilde{\theta}
$$

Agrupando los términos de orden $O\left(W^{-2}\right)$ en la ecuación (E.8), y teniendo en cuenta (D.10), (D.21) y (E.10):

$$
\frac{1}{r^{2}}\left(r^{2} F \tilde{\theta}_{s_{p 0 \pm}}+B_{p 0}\right)-\frac{\partial \tilde{\theta}_{s_{p 0 \pm}}}{\partial t}+\frac{\partial \tilde{\theta}_{s_{p 0 \pm}}}{\partial r} \frac{\mathrm{d} A_{0}}{\mathrm{~d} r}=0 \quad \text { en } \quad \tilde{\theta}=\tilde{\theta}_{s_{s t \pm}}
$$

donde, teniendo en cuenta que $\mathrm{d} A_{0} / \mathrm{d} r=1$ y $F=-2 / r$, y considerando perturbaciones antisimétricas, esto es, $\tilde{\theta}_{s_{p+}}=\tilde{\theta}_{s_{p-}}=\tilde{\theta}_{s_{p}}$, se llega a la siguiente expresión para $B_{p 0}$ en función de $\tilde{\theta}_{s_{p 0}}$ :

$$
B_{p 0}=\frac{\partial\left(r^{2} \tilde{\theta}_{s_{p 0}}\right)}{\partial t}+\frac{\partial\left(r^{2} \tilde{\theta}_{s_{p 0}}\right)}{\partial r}
$$

Por otro lado, tomando los términos de orden $O\left(W^{-4}\right)$ en la expresión (E.7), y teniendo en cuenta (D.10), (D.22) y (E.10):

$$
\frac{1}{r^{2}}\left(r^{2} F \tilde{\theta}+B_{2}\right) B_{p 0}+\frac{\partial A_{p 0}}{\partial t}+\frac{\partial B_{p 0}}{\partial t} \tilde{\theta}+\frac{\mathrm{d} A_{0}}{\mathrm{~d} r}\left(\frac{\partial A_{p 0}}{\partial r}+\frac{\partial B_{p 0}}{\partial r} \tilde{\theta}\right)+p_{p 0}=0
$$

Operando:

$$
\left(\frac{\partial B_{p 0}}{\partial t}+\frac{\partial B_{p 0}}{\partial r}-\frac{2 B_{p 0}}{r}\right) \tilde{\theta}+\frac{B_{2} B_{p 0}}{r^{2}}+\frac{\partial A_{p 0}}{\partial t}+\frac{\partial A_{p 0}}{\partial r}+p_{p 0}=0
$$

de donde, particularizando en las superficies exterior e interior de la lámina líquida:

$$
\begin{gathered}
\left(\frac{\partial B_{p 0}}{\partial t}+\frac{\partial B_{p 0}}{\partial r}-\frac{2 B_{p 0}}{r}\right)\left(\tilde{\theta}_{s_{s t}}+\tilde{\theta}_{s_{p}}\right)+\frac{B_{2} B_{p 0}}{r^{2}}+\frac{\partial A_{p 0}}{\partial t}+\frac{\partial A_{p 0}}{\partial r}+p_{p 0}=0 \\
\left(\frac{\partial B_{p 0}}{\partial t}+\frac{\partial B_{p 0}}{\partial r}-\frac{2 B_{p 0}}{r}\right)\left(-\tilde{\theta}_{s_{s t}}+\tilde{\theta}_{s_{p}}\right)+\frac{B_{2} B_{p 0}}{r^{2}}+\frac{\partial A_{p 0}}{\partial t}+\frac{\partial A_{p 0}}{\partial r}-p_{p 0}=0
\end{gathered}
$$

Restando la expresión (E.13) a (E.12) se obtiene la siguiente relación para $p_{p 0}$ :

$$
p_{p 0}=-\left(\frac{\partial B_{p 0}}{\partial t}+\frac{\partial B_{p 0}}{\partial r}-\frac{2 B_{p 0}}{r}\right) \tilde{\theta}_{s s t}
$$

con $\tilde{\theta}_{s_{s t}}$ dado por (D.34). Introduciendo el valor de $B_{p 0}$ dado por (E.11) en (E.14):

$$
p_{p 0}=-\tilde{\theta}_{s_{s t}}\left(\frac{\partial}{\partial t}+\frac{\partial}{\partial r}-\frac{2}{r}\right)\left(\frac{\partial}{\partial t}+\frac{\partial}{\partial r}\right)\left(r^{2} \tilde{\theta}_{s_{p 0}}\right)
$$

Tomando ahora los términos de orden $O\left(W^{-4}\right)$ de la ecuación (E.9):

$$
p_{p 0}+\frac{\partial p_{s t 2}}{\partial \tilde{\theta}} \tilde{\theta}_{s_{p 0}}-p_{g_{p}}+\frac{1}{r^{2}} \frac{\partial}{\partial r}\left(r^{3} \frac{\partial \tilde{\theta}_{s_{p 0}}}{\partial r}\right)+\frac{\tilde{\theta}_{s_{p 0}}}{r \sin ^{2} \theta_{c}}=0 \quad \text { en } \quad \tilde{\theta}=\tilde{\theta}_{s_{s t \pm}}
$$


donde $p_{s t 2}$ se obtiene de agrupar los términos de orden $O\left(W^{-4}\right)$ en la ecuación (D.7):

$$
\begin{aligned}
& {\left[\left(\frac{\mathrm{d} \theta_{c}}{\mathrm{~d} r}\right)^{2}+\frac{1}{r^{2}}\right] \frac{1}{2}\left[F\left(\left(\frac{\mathrm{d} \theta_{c}}{\mathrm{~d} r}\right)^{2}+\frac{1}{r^{2}}\right)^{-1} \tilde{\theta}+B_{2}\right]^{2}+\frac{1}{2}\left(\frac{\mathrm{d} A_{1}}{\mathrm{~d} r}+\frac{\mathrm{d} B_{1}}{\mathrm{~d} r} \tilde{\theta}\right)^{2}} \\
& +\frac{B_{1}}{r^{2}}\left(\frac{\mathrm{d} \theta_{c}}{\mathrm{~d} r}\right)^{-1} \frac{1}{2}\left[\left(\frac{\mathrm{d} \theta_{c}}{\mathrm{~d} r}\right)^{2}+\frac{1}{r^{2}}\right]^{-2}\left[\frac{\mathrm{d} F}{\mathrm{~d} r}\left(\left(\frac{\mathrm{d} \theta_{c}}{\mathrm{~d} r}\right)^{2}+\frac{1}{r^{2}}\right)-F\left(2 \frac{\mathrm{d} \theta_{c}}{\mathrm{~d} r} \frac{\mathrm{d}^{2} \theta_{c}}{\mathrm{~d} r^{2}}-\frac{2}{r^{3}}\right)\right] \tilde{\theta}^{2} \\
& +\frac{B_{1}}{r^{2}}\left(\frac{\mathrm{d} \theta_{c}}{\mathrm{~d} r}\right)^{-1}\left(\frac{\mathrm{d} B_{2}}{\mathrm{~d} r} \tilde{\theta}+\frac{\mathrm{d} A_{2}}{\mathrm{~d} r}\right)+\left[B_{1}\left(\left(\frac{\mathrm{d} \theta_{c}}{\mathrm{~d} r}\right)^{2}+\frac{1}{r^{2}}\right)-\frac{\mathrm{d} \theta_{c}}{\mathrm{~d} r} \frac{\mathrm{d} A_{0}}{\mathrm{~d} r}\right] \frac{\partial \varphi_{s t 3}}{\partial \tilde{\theta}} \\
& -\frac{\mathrm{d} \theta_{c}}{\mathrm{~d} r}\left(\frac{\mathrm{d} A_{1}}{\mathrm{~d} r}+\frac{\mathrm{d} B_{1}}{\mathrm{~d} r} \tilde{\theta}\right)\left[F\left(\left(\frac{\mathrm{d} \theta_{c}}{\mathrm{~d} r}\right)^{2}+\frac{1}{r^{2}}\right)^{-1} \tilde{\theta}+B_{2}\right]+p_{s t 2}-\frac{\gamma^{2} \tilde{\theta} \cos \theta_{c}}{r^{2} \sin ^{3} \theta_{c}}=0
\end{aligned}
$$

Teniendo en cuenta (D.14), y que $\mathrm{d} \theta_{c} / \mathrm{d} r=0$, la anterior expresión se reduce a:

$$
p_{s t 2}=-\tilde{\theta}^{2}+\left[\frac{2 B_{2}}{r}-\frac{\mathrm{d} B_{2}}{\mathrm{~d} r}+\frac{\gamma^{2} \cos \theta_{c}}{r^{2} \sin ^{3} \theta_{c}}\right] \tilde{\theta}-\frac{B_{2}^{2}}{2 r^{2}}-\frac{\mathrm{d} A_{2}}{\mathrm{~d} r}-\frac{\gamma^{4}}{8 r^{4} \sin ^{4} \theta_{c}}
$$

Como $\partial p_{s t 2} / \partial \tilde{\theta}$ ha de ser impar, entonces:

$$
\frac{2 B_{2}}{r}-\frac{\mathrm{d} B_{2}}{\mathrm{~d} r}+\frac{\gamma^{2} \cos \theta_{c}}{r^{2} \sin ^{3} \theta_{c}}=0
$$

y el término $p_{s t 2}$ del desarrollo de la presión en el líquido queda:

$$
p_{s t 2}=-\tilde{\theta}^{2}-\frac{B_{2}^{2}}{2 r^{2}}-\frac{\mathrm{d} A_{2}}{\mathrm{~d} r}-\frac{\gamma^{4}}{8 r^{4} \sin ^{4} \theta_{c}}
$$

Introduciendo (E.15) y (E.17) en (E.16):

$$
\begin{aligned}
-\frac{c_{d}}{4 r^{2} \sin \theta_{c}} & \left(\frac{\partial}{\partial t}+\frac{\partial}{\partial r}-\frac{2}{r}\right)\left(\frac{\partial}{\partial t}+\frac{\partial}{\partial r}\right)\left(r^{2} \tilde{\theta}_{s_{p 0}}\right) \\
& -\frac{c_{d}}{2 r^{2} \sin \theta_{c}} \tilde{\theta}_{s_{p 0}}-p_{g_{p}}\left(\tilde{\theta}=\tilde{\theta}_{s_{s t}}\right)+\frac{1}{r^{2}} \frac{\partial}{\partial r}\left(r^{3} \frac{\partial \tilde{\theta}_{s_{p 0}}}{\partial r}\right)+\frac{\tilde{\theta}_{s_{p 0}}}{r \sin ^{2} \theta_{c}}=0
\end{aligned}
$$

La longitud de onda de las perturbaciones es larga en comparación con el espesor de la lámina, pero corta en comparación con la extensión de la misma. Como estamos considerando perturbaciones de pequeña amplitud, y su variación es lenta con respecto a $r$, podemos buscar soluciones de este problema con el método WKB. Para ello introducimos una nueva función desconocida, $s(r)$, y una nueva constante, $A$, y expresamos $\tilde{\theta}_{s_{p 0}}$ en términos de ellas mediante la relación:

$$
\tilde{\theta}_{s_{p 0}}=A e^{i s(r)-i \omega t}
$$


donde forzamos con la frecuencia $\omega$ constante. Así pues se tiene:

$$
\begin{aligned}
&\left(\frac{\partial}{\partial t}+\frac{\partial}{\partial r}-\frac{2}{r}\right)\left(\frac{\partial}{\partial t}+\frac{\partial}{\partial r}\right)\left(r^{2} \tilde{\theta}_{s_{p 0}}\right)= \\
& {\left[-r^{2}\left(-\omega+\frac{\mathrm{d} s}{\mathrm{~d} r}\right)^{2}-2+2 i r\left(-\omega+\frac{\mathrm{d} s}{\mathrm{~d} r}\right)+i r^{2} \frac{\mathrm{d}^{2} s}{\mathrm{~d} r^{2}}\right] A e^{i s-i \omega t} } \\
& \frac{1}{r^{2}} \frac{\partial}{\partial r}\left(r^{3} \frac{\partial \tilde{\theta}_{s_{p 0}}}{\partial r}\right)=\left[\frac{1}{r}+3 i \frac{\mathrm{d} s}{\mathrm{~d} r}+i r \frac{\mathrm{d}^{2} s}{\mathrm{~d} r^{2}}-r\left(\frac{\mathrm{d} s}{\mathrm{~d} r}\right)^{2}\right] A e^{i s-i \omega t}
\end{aligned}
$$

Resta por determinar la perturbación en la presión del gas. Las ecuaciones linealizadas que gobiernan el problema son las siguientes:

$$
\begin{gathered}
\frac{1}{r^{2}} \frac{\partial}{\partial r}\left(r^{2} \frac{\partial \varphi_{g_{p}}}{\partial r}\right)+\frac{1}{r^{2} \sin \theta} \frac{\partial}{\partial \theta}\left(\sin \theta \frac{\partial \varphi_{g_{p}}}{\partial \theta}\right)=0 \\
\alpha \frac{\partial \varphi_{g_{p}}}{\partial t}+p_{g_{p}}=0 \\
\frac{\partial \tilde{\theta}_{s_{p \pm}}}{\partial t}+\frac{\partial \tilde{\theta}_{s_{s t \pm}}}{\partial r} \frac{\partial \varphi_{g_{p}}}{\partial r}=\frac{W^{2}}{r^{2}} \frac{\partial \varphi_{g_{p}}}{\partial \theta} \quad \text { en } \quad \theta=\theta_{c}+W^{-2} \tilde{\theta}_{s_{s t \pm}}
\end{gathered}
$$

donde $\alpha=\rho_{g} / \rho$, siendo $\rho_{g}$ la densidad del gas. Introducimos los siguientes desarrollos para las perturbaciones en el potencial de velocidades y en la presión en el gas:

$$
\varphi_{g_{p}}=W^{-2} \varphi_{g_{p 0}}+\ldots \quad ; \quad p_{g_{p}}=W^{-4} p_{g_{p 0}}+\ldots
$$

Introduciendo estos desarrollos en las ecuaciones (E.22) - (E.24), agrupando términos por orden y tomando los principales:

$$
\begin{gathered}
\frac{1}{r^{2}} \frac{\partial}{\partial r}\left(r^{2} \frac{\partial \varphi_{g_{p 0}}}{\partial r}\right)+\frac{1}{r^{2} \sin \theta} \frac{\partial}{\partial \theta}\left(\sin \theta \frac{\partial \varphi_{g_{p 0}}}{\partial \theta}\right)=0 \\
\beta \frac{\partial \varphi_{g_{p 0}}}{\partial t}+p_{g_{p 0}}=0 \\
\frac{\partial \tilde{\theta}_{s_{p 0 \pm}}}{\partial t}=\frac{1}{r^{2}} \frac{\partial \varphi_{g_{p 0}}}{\partial \theta} \text { en } \quad \theta=\theta_{c}
\end{gathered}
$$

donde

$$
\beta=\alpha W^{2} \gg 1
$$

Debido a que estamos considerando perturbaciones de pequeña amplitud, y su variación es lenta en el espacio, podemos aplicar también el método WKB al problema del gas, para lo que introducimos la nueva función desconocida $f(r, \theta)$. Las funciones $s(r)$ y $f(r, \theta)$ admiten desarrollos de la forma $s(r)=s_{0}(r)+s_{1}(r)+\ldots$, con $s_{0}=O(\beta)$ y $s_{1}=O(1)$, 
y $f(r, \theta)=f_{0}(r, \theta)+f_{1}(r, \theta)+\ldots$, con $f_{0}=O(1)$ y $f_{1}=O\left(\beta^{-1}\right)$. Expresamos $\varphi_{g_{p 0}}$ en términos de ella como sigue:

$$
\varphi_{g_{p 0}}=f(r, \theta) e^{i s(r)-i \omega t}
$$

Insertando (E.29) en (E.25):

$$
\frac{2}{r} \frac{\partial f}{\partial r}+\frac{\partial^{2} f}{\partial r^{2}}+i f \frac{\mathrm{d}^{2} s}{\mathrm{~d} r^{2}}+2 i \frac{\mathrm{d} s}{\mathrm{~d} r} \frac{\partial f}{\partial r}+\frac{2 i}{r} f \frac{\mathrm{d} s}{\mathrm{~d} r}-f\left(\frac{\mathrm{d} s}{\mathrm{~d} r}\right)^{2}+\frac{1}{r^{2}} \frac{\partial^{2} f}{\partial \theta^{2}}+\frac{\cot \theta}{r^{2}} \frac{\partial f}{\partial \theta}=0
$$

Desarrollando las funciones $s$ y $f$, y agrupando términos del mismo orden en (E.30):

$$
\begin{gathered}
\frac{1}{r^{2}} \frac{\partial^{2} f_{0}}{\partial \theta^{2}}-\left(\frac{\mathrm{d} s_{0}}{\mathrm{~d} r}\right)^{2} f_{0}=0 \\
\frac{1}{r^{2}} \frac{\partial^{2} f_{1}}{\partial \theta^{2}}-\left(\frac{\mathrm{d} s_{0}}{\mathrm{~d} r}\right)^{2} f_{1}=2 f_{0} \frac{\mathrm{d} s_{0}}{\mathrm{~d} r} \frac{\mathrm{d} s_{1}}{\mathrm{~d} r}-i f_{0} \frac{\mathrm{d}^{2} s_{0}}{\mathrm{~d} r^{2}}-\frac{2 i f_{0}}{r} \frac{\mathrm{d} s_{0}}{\mathrm{~d} r}-2 i \frac{\mathrm{d} s_{0}}{\mathrm{~d} r} \frac{\partial f_{0}}{\partial r}-\frac{\cot \theta_{c}}{r^{2}} \frac{\partial f_{0}}{\partial \theta}
\end{gathered}
$$

Resolviendo (E.31):

$$
f_{0}=B_{g 0} e^{-r \frac{\mathrm{d} s_{0}}{\mathrm{~d} r}\left(\theta-\theta_{c}\right)}
$$

Introduciendo (E.33) en (E.32):

$$
\begin{aligned}
& \frac{1}{r^{2}} \frac{\partial^{2} f_{1}}{\partial \theta^{2}}-\left(\frac{\mathrm{d} s_{0}}{\mathrm{~d} r}\right)^{2} f_{1}= \\
& {\left[2 \frac{\mathrm{d} s_{0}}{\mathrm{~d} r} \frac{\mathrm{d} s_{1}}{\mathrm{~d} r}-i \frac{\mathrm{d}^{2} s_{0}}{\mathrm{~d} r^{2}}-\frac{2 i}{r} \frac{\mathrm{d} s_{0}}{\mathrm{~d} r}+2 i \frac{\mathrm{d} s_{0}}{\mathrm{~d} r} \frac{\mathrm{d}}{\mathrm{d} r}\left(r \frac{\mathrm{d} s_{0}}{\mathrm{~d} r}\right)\left(\theta-\theta_{c}\right)+\frac{\cot \theta_{c}}{r} \frac{\mathrm{d} s_{0}}{\mathrm{~d} r}\right] B_{g 0} e^{-r \frac{\mathrm{d} s_{0}}{\mathrm{~d} r}\left(\theta-\theta_{c}\right)}}
\end{aligned}
$$

Expresamos $f_{1}$ como:

$$
f_{1}=\left[A_{g 1}+B_{g 1}\left(\theta-\theta_{c}\right)+C_{g 1}\left(\theta-\theta_{c}\right)^{2}\right] e^{-r \frac{\mathrm{d} s_{0}}{\mathrm{~d} r}\left(\theta-\theta_{c}\right)}
$$

Llevando (E.35) a (E.34):

$$
\begin{aligned}
&-\frac{4 C_{g 1}}{r} \frac{\mathrm{d} s_{0}}{\mathrm{~d} r}\left(\theta-\theta_{c}\right)+\frac{2 C_{g 1}}{r^{2}}-\frac{2 B_{g 1}}{r} \frac{\mathrm{d} s_{0}}{\mathrm{~d} r}= \\
& {\left[2 i \frac{\mathrm{d} s_{0}}{\mathrm{~d} r} \frac{\mathrm{d}}{\mathrm{d} r}\left(r \frac{\mathrm{d} s_{0}}{\mathrm{~d} r}\right)\left(\theta-\theta_{c}\right)+2 \frac{\mathrm{d} s_{0}}{\mathrm{~d} r} \frac{\mathrm{d} s_{1}}{\mathrm{~d} r}-i \frac{\mathrm{d}^{2} s_{0}}{\mathrm{~d} r^{2}}-\frac{2 i}{r} \frac{\mathrm{d} s_{0}}{\mathrm{~d} r}+\frac{\cot \theta_{c}}{r} \frac{\mathrm{d} s_{0}}{\mathrm{~d} r}\right] B_{g 0} }
\end{aligned}
$$

luego:

$$
\begin{aligned}
& -\frac{4 C_{g 1}}{r} \frac{\mathrm{d} s_{0}}{\mathrm{~d} r}=2 i \frac{\mathrm{d} s_{0}}{\mathrm{~d} r} \frac{\mathrm{d}}{\mathrm{d} r}\left(r \frac{\mathrm{d} s_{0}}{\mathrm{~d} r}\right) B_{g 0} \\
& \frac{2 C_{g 1}}{r^{2}}-\frac{2 B_{g 1}}{r} \frac{\mathrm{d} s_{0}}{\mathrm{~d} r}=\left(2 \frac{\mathrm{d} s_{0}}{\mathrm{~d} r} \frac{\mathrm{d} s_{1}}{\mathrm{~d} r}-i \frac{\mathrm{d}^{2} s_{0}}{\mathrm{~d} r^{2}}-\frac{2 i}{r} \frac{\mathrm{d} s_{0}}{\mathrm{~d} r}+\frac{\cot \theta_{c}}{r} \frac{\mathrm{d} s_{0}}{\mathrm{~d} r}\right) B_{g 0}
\end{aligned}
$$


De (E.36) se obtiene $C_{g 1}$ en función de $B_{g 0}$ :

$$
C_{g 1}=-i \frac{r}{2} \frac{\mathrm{d}}{\mathrm{d} r}\left(r \frac{\mathrm{d} s_{0}}{\mathrm{~d} r}\right) B_{g 0}
$$

e insertando (E.38) en (E.37) se obtiene $B_{g 1}$ en función de $B_{g 0}$ :

$$
B_{g 1}=\left(\frac{i}{2}-r \frac{\mathrm{d} s_{1}}{\mathrm{~d} r}-\frac{\cot \theta_{c}}{2}\right) B_{g 0}
$$

Introduciendo ahora (E.19) y (E.29) en (E.27), y teniendo en cuenta (E.33), (E.35), (E.38) y (E.39):

$$
-i \omega A=\left(\frac{i}{2 r^{2}}-\frac{1}{r} \frac{\mathrm{d} s_{0}}{\mathrm{~d} r}-\frac{1}{r} \frac{\mathrm{d} s_{1}}{\mathrm{~d} r}-\frac{\cot \theta_{c}}{2 r^{2}}\right) B_{g 0}-\frac{1}{r} \frac{\mathrm{d} s_{0}}{\mathrm{~d} r} A_{g 1}
$$

Agrupando términos:

$$
\begin{gathered}
-i \omega A=-\frac{1}{r} \frac{\mathrm{d} s_{0}}{\mathrm{~d} r} B_{g 0} \\
0=\left(\frac{i}{2 r^{2}}-\frac{1}{r} \frac{\mathrm{d} s_{1}}{\mathrm{~d} r}-\frac{\cot \theta_{c}}{2 r^{2}}\right) B_{g 0}-\frac{1}{r} \frac{\mathrm{d} s_{0}}{\mathrm{~d} r} A_{g 1}
\end{gathered}
$$

De (E.40) se obtiene $B_{g 0}$ en función de $A$ :

$$
B_{g 0}=i \omega r\left(\frac{\mathrm{d} s_{0}}{\mathrm{~d} r}\right)^{-1} A
$$

e insertando (E.42) en (E.41) se obtiene $A_{g 1}$ en función de $A$ :

$$
A_{g 1}=i \omega\left(\frac{\mathrm{d} s_{0}}{\mathrm{~d} r}\right)^{-2}\left(\frac{i}{2}-r \frac{\mathrm{d} s_{1}}{\mathrm{~d} r}-\frac{\cot \theta_{c}}{2}\right) A
$$

Particularizando la ecuación (E.26) en $\theta=\theta_{c}$

$$
p_{g_{p 0}}=i \beta \omega\left(B_{g 0}+A_{g 1}\right) e^{i s-i \omega t}
$$

Llevando los valores de $B_{g 0}$ y $A_{g 1}$ dados por (E.42) y (E.43) a (E.44) se obtiene finalmente la expresión para la perturbación de la presión en el gas:

$$
p_{g_{p 0}}=i \beta \omega\left[i r \omega\left(\frac{\mathrm{d} s_{0}}{\mathrm{~d} r}\right)^{-1}+i \omega\left(\frac{\mathrm{d} s_{0}}{\mathrm{~d} r}\right)^{-2}\left(\frac{i}{2}-r \frac{\mathrm{d} s_{1}}{\mathrm{~d} r}-\frac{\cot \theta_{c}}{2}\right)\right] A e^{i s-i \omega t}
$$

Introduciendo (E.19), (E.20), (E.21) y (E.45) en (E.18), desarrollando y agrupando términos del mismo orden:

$$
\frac{c_{d}}{4 \sin \theta_{c}}\left(-\omega+\frac{\mathrm{d} s_{0}}{\mathrm{~d} r}\right)^{2}-r\left(\frac{\mathrm{d} s_{0}}{\mathrm{~d} r}\right)^{2}+\beta r \omega^{2}\left(\frac{\mathrm{d} s_{0}}{\mathrm{~d} r}\right)^{-1}=0
$$




$$
\begin{aligned}
\frac{c_{d}}{4 \sin \theta_{c}}[2(-\omega+ & \left.\left.\frac{\mathrm{d} s_{0}}{\mathrm{~d} r}\right)\left(\frac{\mathrm{d} s_{1}}{\mathrm{~d} r}-\frac{i}{r}\right)-i \frac{\mathrm{d}^{2} s_{0}}{\mathrm{~d} r^{2}}\right]-2 r \frac{\mathrm{d} s_{0}}{\mathrm{~d} r} \frac{\mathrm{d} s_{1}}{\mathrm{~d} r} \\
& +3 i \frac{\mathrm{d} s_{0}}{\mathrm{~d} r}+i r \frac{\mathrm{d}^{2} s_{0}}{\mathrm{~d} r^{2}}+\beta \omega^{2}\left(\frac{\mathrm{d} s_{0}}{\mathrm{~d} r}\right)^{-2}\left(\frac{i}{2}-r \frac{\mathrm{d} s_{1}}{\mathrm{~d} r}-\frac{\cot \theta_{c}}{2}\right)=0
\end{aligned}
$$

De (E.46) se obtiene finalmente la siguiente relación de dispersión:

$$
\left(-\Omega+S^{\prime}\right)^{2} S^{\prime}+X\left(\Omega^{2}-S^{\prime 3}\right)=0
$$

donde:

$$
X=\frac{4 r \sin \theta_{c}}{c_{d}} \quad ; \quad \Omega=\frac{\omega}{\beta} \quad ; \quad S^{\prime}=\frac{1}{\beta} \frac{\mathrm{d} s_{0}}{\mathrm{~d} r}
$$

mientras que de (E.47) se extrae la siguiente expresión para la variación de la amplitud:

$$
\begin{aligned}
{\left[2 S^{\prime 2}\left(-\Omega+S^{\prime}\right)-X\right.} & \left.\left(\Omega^{2}+2 S^{\prime 3}\right)\right] \frac{\mathrm{d} s_{1}}{\mathrm{~d} X} \\
& =2 i \frac{S^{\prime 2}}{X}\left(-\Omega+S^{\prime}\right)+\frac{i}{3}(1-X) \frac{\mathrm{d} S^{\prime 3}}{\mathrm{~d} X}-3 i S^{\prime 3}-\frac{i-\cot \theta_{c}}{2} \Omega
\end{aligned}
$$




\section{Bibliografía}

Abramovich, G. N. 1944 The theory of swirl atomizers. In Industrial Aerodynamics, pp. 114-121. BNT ZAGI (Central Aerodynamic Institute), Moscow.

Asare, H. R., Takahashi, R. K. \& Hoffman, M. A. 1981 Liquid sheet jet experiments: comparison with linear theory. Trans. ASME: J. Fluids Engng 103, 395-604.

Batchelor, G. K. 1967 An Introduction to Fluid Dynamics. Cambridge University Press.

Bayvel, L. P. \& Orzechowski, Z. 1993 Liquid Atomization. Combustion: an international series.

Binnie, A. M. 1949 The passage of a perfect fluid through a critical cross-section or throat. Proc. R. Soc. Lond. A 197, 545-555.

Binnie, A. M. \& Harris, D. P. 1950 The application of boundary layer theory to swirling liquid flow through a nozzle. Q. J. Mech. Appl. Math. 3, 89-106.

Binnie, A. M. \& Hookings, G. A. 1948 Laboratory experiments on whirlpools. Proc. R. Soc. Lond. A 194, 398-415.

Binnie, A. M., Hookings, G. A. \& Kamel, M. Y. M. 1957 The flow of swirling water through a convergent-diverging nozzle. J. Fluid Mech. 3, 261-274.

Bloor, M. I. G. \& Ingham, D. B. 1977 Axially symmetric boundary layers on a finite disk. Phys. Fluids 20, 1228-1233.

Bremond, N., Clanet, C. \& Villermaux, E. 2007 Atomization of undulating liquid sheets. J. Fluid Mech. 585, 421-456.

Bremond, N. \& Villermaux, E. 2006 Atomization by jet impact. J. Fluid Mech. 549, 273-306.

Burggraf, O. R., Stewartson, K. \& Belcher, R. 1971 Boundary layer induced by a potential vortex. Phys. Fluids 14, 1821-1833.

Chinn, J. J. 2009 An appraisal of swirl atomizer inviscid flow analysis. Atomiz. Sprays 19, 263-311.

Clanet, C. \& Villermaux, E. 2002 Life of a smooth liquid sheet. J. Fluid Mech. 462, 307-340. 
Cooke, J. C. 1952 On Pohlhausen's method with application to a swirl problem of Taylor. J. Aero. Sci. 19, 486-490.

Cousin, J. \& Nuglish, H. J. 2001 Modeling of internal flow in high pressure pressure swirl injectors. SAE Paper 2001-01-0963.

Datta, A. \& Som, S. K. 2000 Numerical prediction of air core diameter, coefficient of discharge and spray cone angle of a swirl spray pressure nozzle. Int. J. Heat Fluid Flow 21, 412-419.

Dombrowski, N. \& Hasson, D. 1969 The flow characteristics of swirl (centrifugal) spray pressure nozzles with low viscosity liquids. AIChE J. 15, 604-611.

Dombrowski, N. \& Johns, W. R. 1963 The aerodynamics instability and disintegration of viscous liquid sheets. Chem. Engng Sci. 18, 203-214.

Donjat, D., Estivalezes, J. L., Michau, M. \& Lavegne, G. 2003 Phenomenological study of the pressure swirl atomizer internal flow. Procs. 9th Int. Conference on Liquid Atomization and Spray Systems, Sorrento, Italy, pp. 12-19.

Drazin, P. G. \& Reid, W. H. 1981 Hydrodynamic stability. Cambridge University Press.

Dumouchel, C., Bloor, M. I. G., Dombrowski, N., Ingham, D. B. \& Ledoux, M. 1992 Boundary-layer characteristics of a swirl atomizer. Atomiz. Sprays 2, 225-237.

Fraser, R. P., Eisenklam, P., Dombrowski, N. \& Hasson, D. 1962 Drop formation from rapidly moving liquid sheet. AIChE J. 8, 672-680.

Giffen, E. \& Muraszew, A. 1953 The Atomization of Liquid Fuels. Chapman and Hall, London.

Gordillo, J. M., Lhuissier, H. \& Villermaux, E. 2014 On the cusps bordering liquid sheets. J. Fluid Mech. 754, R1.

Görtler, H. 1940 Über eine dreidimensionale Instabilität laminarer Grenzschichten an konkaven Wänden. Nachr. Ges. Wiss. Göttingen, N. F. 2, No. 1, 1-26.

Gregory, N. \& Walker, W. S. 1950 The effect on transition of isolated surface excrescences in the boundary layer. Rep. Memor. Aero. Res. Coun., Lond. No. 2779, p. 118.

Hagerty, W. W. \& Shea, J. F. 1955 A study of the stability of plane fluid sheets. J. Appl. Mech. 22, 509-514.

Halder, M. R., Dash, S. K. \& Som, S. K. 2003 Influences of nozzle flow and nozzle geometry on the shape and size of an air core in a hollow cone swirl nozzle. Procs. Inst. Mech. Engrs. 217, 207-217.

Hansen, K. G., Madsen, J., Trinh, C. M., Ibsen, C. H., Solberg, T. \& Hjertager, B. H. 2002 A computational and experimental study of the internal flow in scaled pressureswirl atomizer. Procs. 18th Annual Conference on Liquid Atomization and Spray Systems, 
Zaragoza, Spain, paper 058.

Higuera, F. J. 1993 Natural convection below a downward facing horizontal plate. Eur. J. Mech. B 12, 289-311.

Higuera, F. J. 1994 The hydraulic jump in a viscous laminar flow. J. Fluid Mech. 274, 69-92.

Higuera, F. J. \& Liñán, A. 1993 Chocking conditions for nonuniform viscous flows. Phys. Fluids A 5, 768-770.

Higuera, F. J. \& Pereña, A. 2014 Quasi-cylindrical approximation to the swirling flow in an atomizer chamber. J. Fluid Mech. 758, 603-620.

Holtzclaw, D., Sakman, T., Jeng, S. M., Jog, M. A. \& Benjamin, M. A. 1997 Investigation of the flow in a simplex fuel nozzle. AIAA Paper 97-2970.

Horvay, M. \& Leuckel, W. 1986 Experimental and theoretical investigation of swirl nozzles for pressure-jet atomization. Ger. Chem. Eng. 9, 276-283.

Huang, J. C. P. 1970 The break-up of axisymmetric liquid sheets. J. Fluid Mech. 43, 305-319.

Jeng, S. M., Jog, M. A. \& Benjamin, M. A. 1998 Computational and experimental study of liquid sheet emanating from simplex fuel nozzle. AIAA J. 36, 201-207.

Keller, J. B. 1958 Surface waves on water of non-uniform depth. J. Fluid Mech. 4, 607-614.

Keller, J. B. \& Kolodner, I. 1954 Instability of liquid surfaces and the formation of drops. J. Appl. Phys. 25, 918-921.

Kim, I. \& Sirignano, W. A. 2000 Three-dimensional wave distortion and disintegration of thin planar liquid sheets. J. Fluid Mech. 410, 147-183.

Kim, S., Khil, T., Kim, D. \& Yoon, Y. 2009 Effect of geometric parameters on the liquid film thickness and air core formation in a swirl injector. Meas. Sci. Technol. 20, $1-11$.

von Lavante, E., Maatje, U. \& Albina, F. A. 2002 Investigation of unsteady effects in pressure swirl atomizers. Procs. 18th Annual Conference on Liquid Atomization and Spray Systems, Zaragoza, Spain, paper 081.

Lefebvre, A. H. 1989 Atomization and Sprays. Hemisphere. New York.

Lighthill, J. 1978 Waves in fluids. Cambridge University Press.

Lin, S. P. 2003 Breakup of Liquid Sheets and Jets. Cambridge University Press.

Lozano, A., García-Olivares, A. \& Dopazo, C. 1998 The instability growth leading to a liquid sheet break up. Phys. Fluids 10, 2188-2197.

Maatje, U, von Lavante, E. \& Albina, F. A. 2002 Numerical simulations of unsteady 
effects in simplex nozzles. AIAA Paper 2002-3179.

Madsen, J., Hjertager, B. H. \& Solberg, T. 2002 Numerical simulation of internal flow in a large-scale pressure-swirl atomizer. Procs. 18th Annual Conference on Liquid Atomization and Spray Systems, Zaragoza, Spain, paper 028.

Mansour, A. \& Chigier, N. 1990 Disintegration of liquid sheets. Phys. Fluids A 2, 706-719.

Moon, S., Abo-Serie, E. \& Bae, C. 2010 Liquid film thickness inside the high pressure swirl injectors: real scale measurements and evaluation of analytical equations. Exp. Therm. Fluid Sci. 34, 113-121.

Nieuwkamp, W. C. 1985 Flow analysis of a hollow cone nozzle with potential flow theory. Procs. 1st Int. Conference on Liquid Atomization and Spray Systems, London, UK, vol IIIC/1, pp. 1-9.

Nouri-Borujerdi, A. \& Kebriaee, A. 2012 Numerical simulation of laminar and turbulent two-phase flow in pressure-swirl atomizers. AIAA J. 50, 2091-2101.

Novikov, I. I. 1948 Atomization of liquids by centrifugal nozzles. J. Tech. Phys. 18, 345-354.

Park, H. \& Heister, S. D. 2006 Non linear simulation of free surfaces and atomization in pressure swirl atomizers. Phys. Fluids 18, 52103.

Park, J., Huh, K. Y., Li, X. \& Renksizbulut, M. 2004 Experimental investigation on cellular breakup of a planar liquid sheet from an air-blast nozzle. Phys. Fluids 16, 625-632.

Pozrikidis, C. 2002 A practical guide to boundary element methods with the software library BEMLIB. Chapman \& Hall/CRC.

Rayleigh, Lord 1879 On the instability of jets. Proc. Lond. Math. Soc. 4, 10.

Rayleigh, Lord 1916 On the dynamics of revolving fluids. Proc. Roy. Soc. A 93, 148154.

Rizk, N. K. \& Lefebvre, A. H. 1985 Internal flow characteristics of simplex swirl atomizers. J. Propulsion 1, 193-199.

Sakman, A. T., Jog, M. A., Jeng, S. M. \& Benjamin, M. A. 2000 Parametric study of simplex fuel nozzle internal flow and performance. AIAA J. 38, 1214-1218.

Squire, H. B. 1953 Investigation of the stability of a moving liquid film. Brit. J. Appl. Phys. 4, 167-169.

Steinthorsson, E. \& Lee, D. M. 2000 Numerical simulations of internal flow in a simplex atomizer. Procs. 8th Int. Conference on Liquid Atomization and Spray Systems, Pasadena, CA, pp. 324-331.

Suyari, M. \& Lefebvre, A. H. 1986 Film thickness measurements in a simplex swirl 
atomizer. J. Propulsion 2, 528-533.

Taylor, G. I. 1948 The mechanics of swirl atomizers. Proc. 7th Int. Congress Appl. Mechanics, vol. 2, pp. 280-285.

Taylor, G. I. 1950a The boundary layer in the converging nozzle of a swirl atomizer. Q. J. Mech. Appl. Math. 3, 129-139.

Taylor, G. I. 1950b The instability of liquid surfaces when accelerated in a direction perpendicular to their planes. I. Proc. R. Soc. Lond. A 202, 192-196.

Taylor, G. I. 1960 Formation of thin flat sheets of water. Proc. R. Soc. Lond. A 259, $1-17$.

Tratnig, A. \& Brenn, G. 2010 Drop size spectra in sprays from pressure-swirl atomizers. Int. J. Multiphase Flow 36, 349-363.

Villermaux, E. \& Clanet, C. 2002 Life of a flapping liquid sheet. J. Fluid Mech. 462, 341-363.

Wang, D., Ma, Z., Jeng, S. M. \& Benjamin, M. A. 1999 Experimental study on largescale simplex nozzle. AIAA Paper A99-31198.

Wang, X. F. \& Lefebvre, A. H. 1987 Mean drop sizes from pressure-swirl nozzles. J. Propulsion 3, 11-18.

Weihs, D. 1978 Stability of thin, radially moving liquid sheets. J. Fluid Mech. 87, 289-298.

Wimmer, E. \& Brenn, G. 2013 Viscous flow through the swirl chamber of a pressureswirl atomizer. Int. J. Multiphase Flow 53, 100-113.

Xue, J., Jog, M. A., Jeng, S. M., Steinthorsson, E. \& Benjamin, M. A. 2004 Effect of geometric parameters on simplex atomizer performance. 35th Joint Propulsion Conference and Exhibit. Los Angeles, CA.

York, J. L., Stubbs, H. E. \& Tek, M. R. 1953 The mechanism of disintegration of liquid sheets. Trans. ASME, 1279-1286.

Yule, A. J. \& Chinn, J. J. 2000 The internal flow structure and exit conditions of pressure swirl atomizers. Atomiz. Sprays 10, 121-146.

Yule, A. J. \& Widger, I. R. 1996 Swirl atomizers operating at high water pressure. Int. J. Mech. Sci. 38, 981-999. 